Universidad Politécnica de Madrid

FACULTAD INFORMÁTICA

\title{
Modeling AND ANALYZING the KINETIC OF CONJUGATIVE PLASMIDS
}

\section{TESIS DOCTORAL}

\author{
Autor \\ Antônio Prestes García, MSc \\ DiRECTOR \\ Alfonso Rodríguez-Patón, PhD
}

MADRID,JUNIO DE 2017 



\section{Dedication}

Este trabalho está dedicado à minha família em geral, aos meus pais e irmãos, aos meus tios e primos, tanto aos inatos como aos adquiridos. Mas em particular meu agradecimento à minha companheira Encarni Fernandez que estoicamente me apoiou e aguentou o meu cansaço e mau humor tudo durante os anos de elaboração dessa tese. Sem a sua ajuda e a estabilidade proporcionada, jamais poderia haver aguentado tanto tempo sem nenhum descanso. Aos tios Renato e Dulviaci. Ao tio Machado pelos seus churrascos na praia. Ao velho pelos verões sem fim de campeiradas na estancia e rodeios bem parados no coxilhão do potreiro. E por último também lembrar de minha vó Dora e agradecer de forma especifica à minha mãe Carmo e à minha tia Graça, todos por terem feito verdade para mim as palavras de Rilke que asseguram que a verdadeira pátria de todo ser humano é a sua infância. 



\section{Acknowledgements}

I would like to express my sincere gratitude to the professor Alfonso RodríguezPatón for his open mind and of his encouraging words but above all for opening the door of his lab to me and accepting to supervise a guy who was planning to undertake his $\mathrm{PhD}$ in a part-time fashion using just late hours and weekends, even when the statistics says that the people who are already working for the industry, rarely are able to complete their thesis.

We also need to thanks the Professor Fernando de la Cruz and the people of the Intergenomics Group which kindly provided the experimental data without which, this work would not have been possible.

I would also like to say some warm words of appreciation and recognition for the Professors João Baptista da Silva and Paulo Silveira Junior from the mathematical and statistics department of UFPEL, who years ago gave me a scholarship for doing research and for telling me the importance of scientific research at all levels. I would also want to mention the Professor Frank Siewerdt for his superb classes.

Finally, a sincere gratitude for who taught me to read, my first teacher "Dona Yolanda". 



\section{Resumen}

La conjugación bacteriana, a pesar de la gran cantidad de información disponible sobre sus aspectos moleculares, todavía no dispone de una forma sistémica estructurada que esté completamente establecida y que además pueda permitir una aproximación en cierto modo estándar a las tareas de construcción de modelos y su simulación computacional. Consecuentemente, ese estado de cosas implica que el modelado de la conjugación sea normalmente complicado, repetitivo, poco fiable y extremamente difícil de ser reproducido por diferentes grupos de investigación. Usando una metáfora computacional, se podría decir que el modelado de la conjugación bacteriana se encuentra en una etapa similar a la era anterior a la programación estructurada haciendo de la comprensión de cualquier implementación en particular, una tarea bastante complicada además de impedir la comparación directa de resultados generados por distintos modelos. Todo ello, obviamente obstaculiza bastante el avance en el aprovechamiento de la conjugación bacteriana como una herramienta para la realización de tareas computacionales a través del uso de los plásmidos como código móvil. Incluso una cuestión tan fundamental sobre cómo se debería representar el proceso de la conjugación en un modelo basado en individuos, todavía necesita ser respondida. El problema empeora por el hecho de que los grupos de investigación en biología sintética normalmente tiene una base formativa diversa y son interdisciplinares por su naturaleza de modo que es complicado producir una visión homogénea sobre la cuestión debido principalmente a que investigadores de diferentes dominios del conocimiento emplean un vocabulario distinto y disponen de un marco mental distinto para definir el proceso bajo escrutinio. 
Disponer de una visión sistémica de cualquier tipo de procesos es normalmente más que una suma simple del conocimiento acumulado sobre las partes del mismo, cosa que en el caso particular de este trabajo se corresponde a la descripción molecular y los aspectos relacionados con la fisiología del ciclo de vida bacteriano. La acción de generar una representación abstracta para el modelado de un sistema es lo que proporciona la visión holística de sistema permitiendo colocar el foco en un punto concreto del sistema, pero siempre contemplando la globalidad por lo menos de aquellos aspectos más significativos. El formalismo de modelado empleado, en sí mismo, para la captura de las propiedades del sistema impone los límites de lo que puede ser estudiado, observado y las cuestiones que se pueden responder con un determinado modelo. Este es precisamente uno de los aspectos más interesantes del modelado basado en individuos que obliga a que en el modelado se tenga en cuenta los detalles internos de la entidad que está siendo representada además de las restricciones temporales y el orden de los eventos. Eso proporciona bastante más información y permite conjeturar muchas más hipótesis sobre la estructura interna de cada célula individual comparándolo con modelos basados en ecuaciones diferenciales.

En esta tesis introducimos un modelo basado en individuos para el proceso de la conjugación bacteriana que tiene como punto central el enlace que existe, de forma plausible, entre ciclo celular bacteriano y el instante temporal en el que la transferencia conjugativa es más susceptible de ocurrir. El modelo ha sido validado comparando los indicadores producidos como salida del modelo con los resultados experimentales de la cinética conjugativa de diferentes tipos de plásmidos. Adicionalmente, el modelo ha sido evaluado usando la metodología conocida como análisis de la sensibilidad global que permite alcanzar una mejor comprensión de la incertidumbre en los parámetros del modelo y el efecto de las formas alternativas de representar los eventos de la transferencia genética horizontal.

El análisis que se ha ejecutado parece soportar que se asuma que la dinámi- 
ca de la conjugación está dominada por el parámetro que representa en momento temporal en que el evento conjugativo tiene lugar. Esa conclusión se asienta sobre dos puntos fundamentales: Uno de ellos es el hecho de que entre las tres diferentes implementaciones realizadas, la que mejor se ajusta a los datos experimentales es la que tiene en cuenta el ciclo celular. El segundo punto es el análisis de la sensibilidad realizado, cuyos resultados apuntan a ese parámetro como significativo.

En el desarrollo de esta tesis hemos implementado el simulador BactoSIM usando el entorno Repast además los paquetes para GNU R, EvoPER y R/Repast para la ejecución de los experimentos. Estos módulos tienen un carácter general y facilitan la realización de experimentos y análisis complejos de forma sencilla. Todo el software creado en este trabajo está disponible como software libre. Como contribución más general este trabajo puede servir a otros como guía para la implementación de un análisis sistémico de los modelos basados en individuos. 



\section{Abstract}

The bacterial conjugation, despite of the amount of molecular information available about its molecular aspects, does not have yet a well-established and structured systemic form which could allow a standard approach to the model building and the computational simulation task. This state of things implies that the modeling task are normally hard, repetitive, unreliable and extremely difficult to reproduce elsewhere by other research groups. Using a computational metaphor, we could state that bacterial conjugation is in a pre-structured programing era which makes the understanding of any particular implementation a very hard task and it also prevents the comparison of results from other models. This obviously hinders a leap forward in the use of the bacterial conjugation as a tool for doing computations with plasmid with plasmid encode mobile code. Even a very basic question, such as how should the conjugation process be represented in an individual-based model is still waiting for being answered. The problem also gets worst because the different backgrounds of synthetic biology research groups, which are interdisciplinary in their very nature, making hard to produce a homogenous view about the question mainly owing to the fact that the people from different domains use different vocabulary and have a different mental framework for understanding the process under study.

The systemic view for any kind of process is much more than a simple accumulation of knowledge about system parts, which in the particular case of conjugation, is represented by the molecular and physiological aspects of bacterial life cycle. The action of generating an abstract representation modeling a sys- 
tem is what provides an holistic systemic view allowing to put focus on a precise point of the system but always taking into account the totality of the significant aspects. The modeling formalism itself for capturing the system properties also imposes limitations on the what can be observed or studied. In other words, the focus and the questions which can be answered are somehow dependent on the granularity of the modeling formalism. That is one of the greatest beauties of the individual-based formalism which forces the modeler to be concerned on the internal details of the entity being modeled as well as the time constraints and the order of the events. These factors provide much more information and allow to make conjectures about the internal strucure of individual bacterial cells when compared, for instance, to an ordinary differential equation model which can only expose the whole-population properies.

In this thesis, we introduce an individual-based model for the conjugation process using, as the central point, the plausible link between the bacterial cell cycle and the time when the conjugative event is most likely to happen. The model was validated comparing the simulation outputs with the experimental data for different types of plasmids. Additionally, the model was assessed using the global sensitivity analysis methodology for providing a better understanding about the parameter uncertainty and the alternative model structures for representing the horizontal genetic transfer event.

The thorough analysis seems to support the assumption that the conjugation dynamics is dominated by the parameter representing the time when the lateral gene transfer takes place. This conclusion is settled over two fundamental aspects. The first is related to the fact that, among the possible representations with respect to the temporal aspects, the best fit to the experimental data is achieved by the logic which takes into account the cell cycle. The second point is the sensitivity analysis which also indicates this parameter as significant.

During the development of this thesis we had implemented the individualbased mode BactoSIM using the Repast framework. We had also implemented the GNU R packages EvoPER and R/Repast for the experimental setup and ana- 
lysis. The tools developed for this work are available as opensource software. Moreover, a lateral contribution of this work is serving as a guide for other modelers in the systematic evaluation of their models. 



\section{Publications and Software}

- Prestes García, A and Rodríguez-Patón, A - A Preliminary Assessment of Three Strategies for the Agent-Based Modeling of Bacterial Conjugation. 9th International Conference on Practical Applications of Computational Biology and Bioinformatics. Advances in Intelligent Systems and Computing, vol. 375,Springer International Publishing, 2015.

- Prestes García, A and Rodríguez-Patón, A - BactoSim - An IndividualBased Simulation Environment for Bacterial Conjugation. 13th International Conference on Practical Applications of Agents and Multi-Agent Systems. Springer International Publishing, 2015.

- Prestes García, A and Rodríguez-Patón, A - Sensitivity analysis of Repast computational ecology models with R/Repast. Ecology and Evolution, Volume 6, Issue 24. John Wiley \& Sons, Ltd, 2016. (JCR)

- Prestes García, A and Rodríguez-Patón, A - EvoPER - An R package for applying evolutionary computation methods in the parameter estimation of individual-based models implemented in Repast. (2016) PeerJ Preprints 4:e2279v1 https://doi.org/10.7287/peerj.preprints.2279v1. 
- Evolutionary Metaheuristics for Parameter Estimation of Individual-Based Models. Submited 2017 (JCR).

- Prestes García, A and Rodríguez-Patón, A - evoper: Evolutionary Parameter Estimation for 'Repast Simphony' Models. https://CRAN.R-project. org/package=evoper (software).

- Prestes García, A and Rodríguez-Patón, A - rrepast: Running 'Repast Simphony' models inside $\mathbf{R}$ environment. https://CRAN.R-project.org/ package $=$ rrepast (software).

- Prestes García, A and Rodríguez-Patón, A - BactoSIM: Individual-based simulation of bacterial conjugation. https://goo.gl/Jo4Fzj(software).

- Prestes García, A and Rodríguez-Patón, A - T4SS Common Pool: Individual-based simulation of two plasmids sharing a common T4SS system. https://g0o.gl/jbAX5w (software). 


\section{Contents}

\begin{tabular}{ll}
\hline Introduction & 1
\end{tabular}

1 Introduction 3

1.1 Overview . . . . . . . . . . . . . . . . 4

1.2 Motivation . . . . . . . . . . . . . . . . . . . 6

1.3 Objectives and Contributions $\ldots \ldots \ldots \ldots \ldots \ldots$

1.4 Document organization $\ldots \ldots \ldots \ldots \ldots \ldots \ldots$

II State of the Art 13

2 Sistemic analysis of bacterial cells $\quad 15$

2.1 Introduction . . . . . . . . . . . . . . . . . . . . 17

2.2 The Bacterial Cell . . . . . . . . . . . . . . . . . . . . . . . . . 19

2.3 The Cell Envelope . . . . . . . . . . . . . . . . . . . . . . 21

2.4 Shape, Morphology and Dimensions . . . . . . . . . . . . . 22

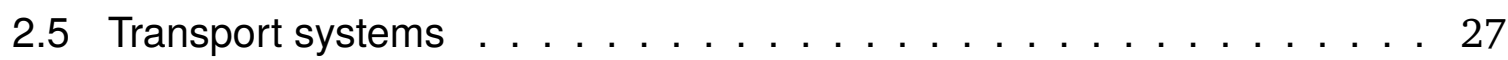

2.6 Secretion systems $\ldots \ldots \ldots \ldots$

2.7 The SOS response . . . . . . . . . . . . . . . . . . . . . . . . . . . . . . . 29

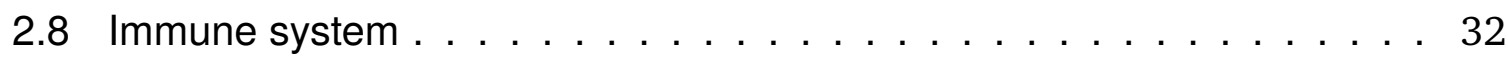

2.8 .1 Restriction Enzymes . . . . . . . . . . . . . . . . . . 32

2.8 .2 The CRISPR/Cas System . . . . . . . . . . . . . . . . . 33

2.9 The DNA Replication . . . . . . . . . . . . . . . . . . . . . 33

2.10 Energy budget $\ldots \ldots \ldots \ldots \ldots \ldots$ 
2.10 .1 The cost of conjugation $\ldots \ldots \ldots \ldots \ldots \ldots$

2.11 Plasmids and conjugation $\ldots \ldots \ldots \ldots . \ldots . \ldots . \ldots . \ldots$

2.12 Summary . . . . . . . . . . . . . . . . . . . . . . . . . 39

3 Models and Conjugation 44

3.1 Overview . . . . . . . . . . . . . . . . . . . 42

3.2 Whole population models of conjugation . . . . . . . . . . . . 42

3.3 Individual-based models of bacterial growth . . . . . . . . . . . 45

3.4 Individual-based models of conjugation . . . . . . . . . . . 49

III The Research Problem and the Methodology 53

4 The challenging of modeling the bacterial conjugation 55

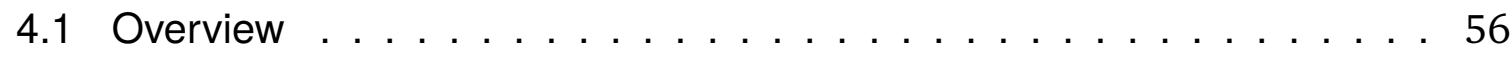

4.2 The research problem . . . . . . . . . . . . . . . 57

4.3 Methodology . . . . . . . . . . . . . . . . . . . . . . . 59

IV The Proposed Solutions to the Research Problem 61

5 Parameter estimation 63

5.1 Introduction . . . . . . . . . . . . . . . . . . . . . 64

5.2 Parameter Estimation and Optimization . . . . . . . . . . 65

5.3 Metaheuristics for Parameter Estimation . . . . . . . . . . . . 70

5.4 Discussion . . . . . . . . . . . . . . . . . . . 85

5.4 .1 Optimizing simple functions $\ldots \ldots \ldots \ldots$. . . . . . 86

5.4 .2 Tuning oscillations $\ldots \ldots \ldots \ldots \ldots$. . . . . . . . . 87

5.4 .3 Exploring the solution space $\ldots \ldots \ldots \ldots$

5.4 .4 Comparing metaheuristics . . . . . . . . . . . . . . 93

5.4 .5 Parameter estimation of individual-based models . . . . . . . 96

5.5 Summary . . . . . . . . . . . . . . . . . . . . . . 107

6 Sensitivity analysis of individual-based models 109

6.1 Introduction . . . . . . . . . . . . . . . . . . . . 111 
6.1 .1 Model development . . . . . . . . . . . . . . . . . . . 114

6.1 .2 Sensitivity analysis . . . . . . . . . . . . . . . . . 116

6.2 Overview of R/Repast package . . . . . . . . . . . . . . . . 123

6.2 .1 Design . . . . . . . . . . . . . . . . . . . . . . . . 124

6.2 .2 The R/Repast R API . . . . . . . . . . . . . . . . . 126

6.2 .3 The objective function interface . . . . . . . . . . . . 131

6.3 Examples overview . . . . . . . . . . . . . . . . . . . . . . 132

6.4 Example 1: BactoSIM . . . . . . . . . . . . . . . . . . . . 134

6.4 .1 Model analysis . . . . . . . . . . . . . . . . . . . 135

6.5 Example 2: Predator-Prey . . . . . . . . . . . . . . . . 136

6.5 .1 Model description . . . . . . . . . . . . . . . 136

6.5 .2 Entities, State variables and scales. . . . . . . . . . . 137

6.5 .3 Model analysis . . . . . . . . . . . . . . . . . . . . . . 139

6.6 Example 3: T4SS Common Pool . . . . . . . . . . . . . . . . . 142

6.6 .1 Model description . . . . . . . . . . . . . . . . . 142

6.6 .2 Process overview and scheduling . . . . . . . . . . . 143

6.6 .3 Analysis of model . . . . . . . . . . . . . . 145

6.7 Summary . . . . . . . . . . . . . . . . . . . 147

7 An individual-based model of bacterial conjugation 151

7.1 Introduction . . . . . . . . . . . . . . . . . . . . . . . 152

7.2 High-level model description. . . . . . . . . . . . . . . . . 153

7.3 Material and methods . . . . . . . . . . . . . . . . . . 157

7.3 .1 Purpose . . . . . . . . . . . . . . . . . . . . . . 157

7.3 .2 State variables and scales. . . . . . . . . . . . . . . . 157

7.3.3 Process overview and scheduling . . . . . . . . . . 158

7.3 .4 Design concepts . . . . . . . . . . . . . . . . . . . 158

7.3 .5 Initialization . . . . . . . . . . . . . . . . . 161

7.3 .6 sub-models . . . . . . . . . . . . . . . . . . 163

7.4 Results and discussion $\ldots \ldots \ldots \ldots \ldots$

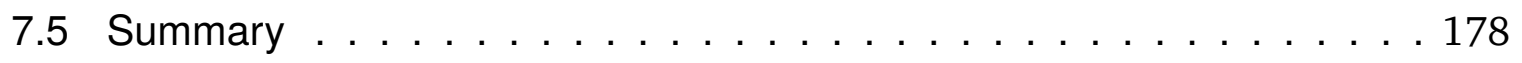


8 Analysis and discusion of the model output 181

8.1 Overview . . . . . . . . . . . . . . . . . . . . . 182

8.2 Definitions . . . . . . . . . . . . . . . . . . . . . . . . 183

8.3 The effect of temporal structure . . . . . . . . . . . . . . . . . . . . 184

8.4 The effect of cell density on initial contact delay . . . . . . . . . . . 191

8.5 Description of model analysis . . . . . . . . . . . . . . . . . . 194

8.6 Preliminary screening of model output $\ldots \ldots \ldots \ldots$

8.7 Assessing the fitness of different models . . . . . . . . . . . . . 208

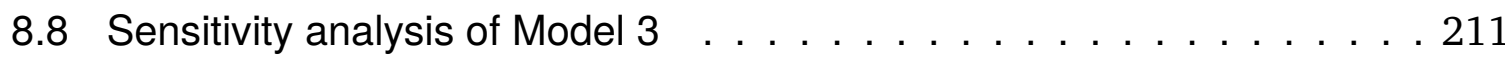

8.8 .1 Sobol indices for a repressed plasmid . . . . . . . . . . . . 213

8.8 .2 Sobol indices for a de-repressed plasmid . . . . . . . . . . . 217

8.8.3 Sobol indices for a mobilizable plasmid . . . . . . . . . . . . . 223

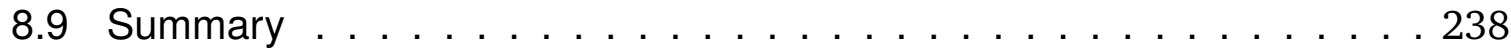

V Conclusions and Future Research 241

9 Conclusions 243

\begin{tabular}{ll}
\hline 10 Future work & 247
\end{tabular}

\begin{tabular}{ll}
\hline Bibliography & 251
\end{tabular}

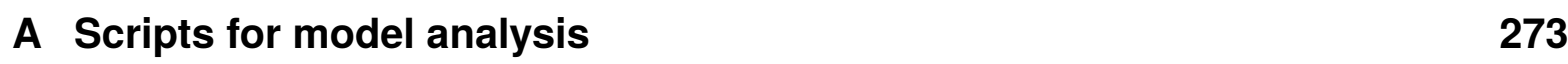




\section{List of Figures}

2.1 The bacterial components . . . . . . . . . . . . . . . . 20

2.2 Envelope volume approximation $\ldots \ldots \ldots \ldots \ldots$

2.3 Shape effect on growth $\ldots \ldots \ldots \ldots \ldots \ldots$

2.4 Transport systems schematics $\ldots \ldots \ldots$

2.5 The SOS response regulatory network $\ldots \ldots \ldots$. . . . . . . . 31

$5.1 \quad$ Example values of $P(\mu)^{k}$ for ees-1 metaheuristic $\ldots \ldots \ldots \ldots 73$

5.2 Implementing a neighborhood function for PSO . . . . . . . . . 84

5.3 Example on how to run a plain function $\ldots \ldots \ldots \ldots$. . . . . . 86

5.4 Output of applying PSO to a plain function . . . . . . . . . . . 87

5.5 Example of tuning oscillations . . . . . . . . . . . . . . . . . . 89

5.6 Ouput of parameter estimation for tuning oscillations . . . . . . . . 9 90

5.7 Tuning oscillations with four different periods $\ldots \ldots \ldots$. . . . . . 91

5.8 Exploring solution space for Rosenbrock/4 . . . . . . . . . . . . . . 92

5.9 Solution space for Rosenbrock/4 using ACO . . . . . . . . . . . . . . 100

5.10 Solution space for Rosenbrock/4 using ees-2 . . . . . . . . . . . . 101

5.11 Comparing objective number function evaluations . . . . . . . . . . 102

5.12 Comparing the fitness value of objective function . . . . . . . . 103

5.13 Parameter estimation of $\mathrm{lbM} . \ldots \ldots \ldots$

5.14 Exploring lbM solution space $\ldots \ldots \ldots$. . . . . . . . . . 105

5.15 Refining lbM solution space $\ldots \ldots \ldots$. . . . . . . . . . . 106

6.1 The iterative model development . . . . . . . . . . . . . . . . . 114

6.2 The different types of sensitivity analysis . . . . . . . . . . . . 120 
6.3 Wrapping an individual-based model . . . . . . . . . . . . . 125

6.4 Implementing an objective function . . . . . . . . . . . . . . 132

6.5 Example code for model output stability . . . . . . . . . . . 136

6.6 The model output stability $\ldots \ldots \ldots$. . . . . . . . . . . . . 137

6.7 Applying the Morris screening method . . . . . . . . . . . . . . 140

6.8 The $\mu^{*}$ and $\sigma$ output of Morris screening method $\ldots \ldots \ldots \ldots 14$

6.9 The $\mu$ and $\sigma$ output of Morris screening method . . . . . . . . . 141

6.10 The $\mu^{*}$ and $\mu$ output of Morris screening method . . . . . . . . 142

6.11 Applying the Sobol method . . . . . . . . . . . . . . . 145

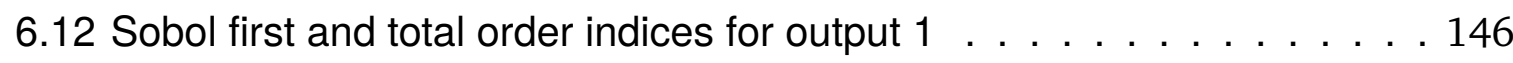

6.13 Sobol first and total order indices for output $2 \ldots \ldots . . .147$

6.14 Sobol first and total order indices for output $3 \ldots \ldots \ldots$

7.1 The BactoSIM Virtual Agar-Plate . . . . . . . . . . . . . . . 154

7.2 Sample output for bactosim . . . . . . . . . . . . . . . . 156

7.3 BactoSIM process scheduling . . . . . . . . . . . . . . . . . . . . . . 159

7.4 Comparing strategies for plasmid pAR118 . . . . . . . . . . . 172

7.5 Comparing strategies for plasmid pSU2007 . . . . . . . . . . 173

7.6 BactoSIM output (pSU2007, $\left.\gamma_{0}\right) \ldots \ldots \ldots \ldots \ldots \ldots$

7.7 BactoSIM output (pSU2007, $\left.R_{0}\right) \ldots \ldots \ldots \ldots \ldots \ldots$

7.8 BactoSIM output (pSU2007, Horizontal infections) $\ldots$. . . . . . . . 177

8.1 Conjugation delays . . . . . . . . . . . . . . . . . . . . 187

8.2 Interaction graph $\ldots \ldots \ldots \ldots$. . . . . . . . . . . . . . . . . . 189

8.3 Forwarding delay effect . . . . . . . . . . . . . . . . . . . . . . 190

8.4 The effect of initial density $\ldots \ldots \ldots$

8.5 Initial density $\left(N_{0}\right)$ effect $\ldots \ldots \ldots \ldots \ldots \ldots$

8.6 Case 1: The Morris metric $\mu$ for $T /(T+R)$ output. . . . . . . . . . . 202

8.7 Case 1: The Morris metric $\mu^{*}$ for $T /(T+R)$ output $\ldots \ldots \ldots \ldots . . .203$

8.8 Case 2: The Morris metric $\mu$ for $T /(T+R)$ output. . . . . . . . . 204

8.9 Case 2: The Morris metric $\mu^{*}$ for $T /(T+R)$ output $\ldots \ldots \ldots \ldots$. . . 205

8.10 Case 3: The Morris metric $\mu$ for $T /(T+R)$ output. . . . . . . . . 206

8.11 Case 3: The Morris metric $\mu^{*}$ for $T /(T+R)$ output . . . . . . . . . 207

8.12 Sobol indices for $T /(T+R) /$ pAR118(5\%) $\ldots \ldots \ldots . . . . .214$ 
8.13 Sobol indices for $D$ doubling time/pAR118(5\%) $\ldots \ldots \ldots$. . . . . 215

8.14 Sobol indices for $T$ doubling time/pAR118(5\%) . . . . . . . . . . . 216

8.15 Sobol indices for composite effect/pAR118(5\%) . . . . . . . . . . . 217

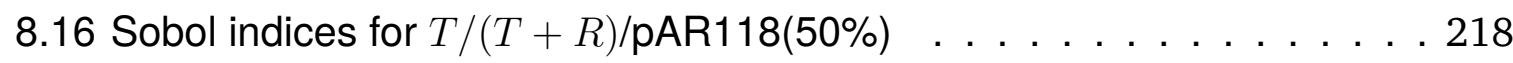

8.17 Sobol indices for $D$ doubling time/pAR118(50\%) . . . . . . . . . . . 219

8.18 Sobol indices for $T$ doubling time/pAR118(50\%) . . . . . . . . . . 220

8.19 Sobol indices for composite effect/pAR118(50\%) . . . . . . . . . . 221

8.20 Sobol indices for $T /(T+R) / \mathrm{pSU} 2007(5 \%) \ldots \ldots . \ldots . \ldots 222$

8.21 Sobol indices for $D$ doubling time/pSU2007(5\%) . . . . . . . . . . 223

8.22 Sobol indices for $T$ doubling time/pSU2007(5\%) . . . . . . . . . . . 224

8.23 Sobol indices for composite effect/pSU2007(5\%) . . . . . . . . . . . 225

8.24 Sobol indices for $T /(T+R) / \mathrm{pSU} 2007(50 \%) \ldots \ldots \ldots . \ldots . . \ldots 226$

8.25 Sobol indices for $D$ doubling time/pSU2007(50\%) . . . . . . . . . . . 227

8.26 Sobol indices for $T$ doubling time/pSU2007(50\%) . . . . . . . . . . . 228

8.27 Sobol indices for composite effect/pSU2007(50\%) . . . . . . . . . . . 229

8.28 Sobol indices for $T /(T+R) /$ pR388(OriT)(5\%) . . . . . . . . . . 230

8.29 Sobol indices for $D$ doubling time/pR388(OriT)(5\%) $\ldots \ldots \ldots . . .231$

8.30 Sobol indices for $T$ doubling time/pR388(OriT)(5\%) . . . . . . . . . . . 232

8.31 Sobol indices for composite effect/pR388(OriT)(5\%) . . . . . . . . . 233

8.32 Sobol indices for $T /(T+R) / \mathrm{pR} 388($ OriT)(50\%) $\ldots \ldots \ldots . . . . . .234$

8.33 Sobol indices for $D$ doubling time/pR388(OriT)(50\%) . . . . . . . . . 235

8.34 Sobol indices for $T$ doubling time/pR388(OriT)(50\%) . . . . . . . . . 236

8.35 Sobol indices for composite effect/pR388(OriT)(50\%) . . . . . . . . . . 237

A.1 Case 1: Script for preliminary screening of BactoSIM . . . . . . . . 275

A.2 Case 2: Script for preliminary screening of BactoSIM . . . . . . . . 276

A.3 Case 3: Script for preliminary screening of BactoSIM . . . . . . . . . 277

A.4 Model 1: Preliminary parameter estimation for BactoSIM . . . . . . . 278

A.5 Model 2: Preliminary parameter estimation for BactoSIM . . . . . . . . 279

A.6 Model 3: Preliminary parameter estimation for BactoSIM . . . . . . . . 280

A.7 Analysis of BactoSIM model cost . . . . . . . . . . . . . . . . 281

A.8 Initial density effect . . . . . . . . . . . . . . . . . . . . . . . 282

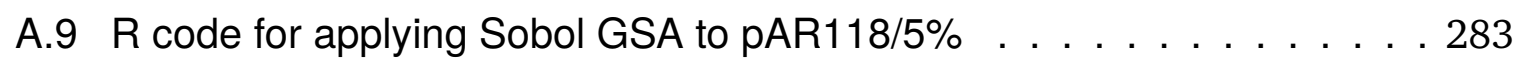


A.10 R code for applying Sobol GSA to pAR118/50\% . . . . . . . . . . 284

A.11 R code for applying Sobol GSA to pSU2007/5\% . . . . . . . . . . 285

A.12 R code for applying Sobol GSA to pSU2007/50\% . . . . . . . . . . 286

A.13 R code for applying Sobol GSA to pR388(OriT)/5\% . . . . . . . . . 287

A.14 R code for applying Sobol GSA to pR388(OriT)/50\% . . . . . . . . . 288 


\section{List of Tables}

2.1 The dimensions of different cell types $\ldots \ldots \ldots \ldots \ldots$

2.2 Parameters for envelope elongation and growth . . . . . . . . . . 27

5.1 Tuning oscillations with particle swarm optimization . . . . . . . 88

5.2 Benchmarking metaheuristics algorithms $\ldots \ldots \ldots \ldots \ldots$

6.1 Example: Predator-pray parameters . . . . . . . . . . . . . 138

6.2 Example: Common plasmid pool paramaters . . . . . . . . . . . . 143

7.1 BactoSIM 1.0 initialization parameters . . . . . . . . . . . . . 162

7.2 Polynomial equations fitted to the experimental data . . . . . . . . 163

7.3 Simulated plasmid parameters . . . . . . . . . . . . . . 171

8.1 Additional BactosIM input parameters . . . . . . . . . . . . . 196

8.2 Case 1: Morris metrics for BactoSIM . . . . . . . . . . . . . . 198

8.3 Case 2: Morris metrics for BactoSIM . . . . . . . . . . . . . . . . . 198

8.4 Case 3: Morris metrics for BactoSIM . . . . . . . . . . . . . . . . . . . . 199

8.5 Case 1: Parameter estimation results for BactoSIM . . . . . . . . . . . . 209

8.6 Case 1: Anova of Parameter estimation results . . . . . . . . . . 210 



\section{List of Algorithms}

2.1 Envelope growth and elongation $\ldots \ldots \ldots \ldots \ldots$

5.1 The outline of an Evolutionary Strategy $\ldots \ldots \ldots$

5.2 The Evoper Evolutionary Strategy-1 . . . . . . . . . . . . . . 75

5.3 The Evoper Evolutionary Strategy-2 $\ldots \ldots \ldots \ldots \ldots$

7.1 The logic for nutrient uptake $\ldots \ldots \ldots \ldots \ldots$

7.2 The time-based division logic $\ldots \ldots \ldots \ldots \ldots \ldots$

7.3 The logic for conjugation strategy $1 \ldots \ldots \ldots \ldots$

7.4 The logic for conjugation strategy $2 \ldots \ldots \ldots$

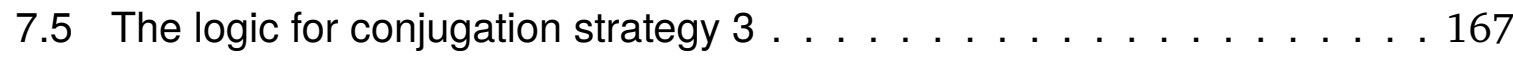

7.6 The logic for expressing the conjugative machinery . . . . . . . 170 



\section{Part I}

\section{Introduction}





\section{Chapter}

\section{Introduction}

1.1 Overview . . . . . . . . . . . . . . . 4

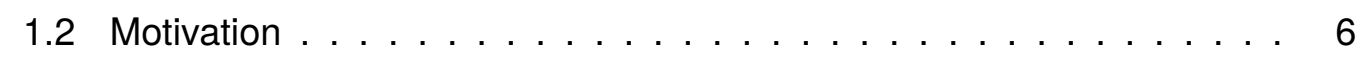

1.3 Objectives and Contributions $\ldots \ldots \ldots \ldots \ldots \ldots$

1.4 Document organization $\ldots \ldots \ldots \ldots \ldots \ldots \ldots \ldots$ 


\subsection{Overview}

The study of living systems is a history told in terms of diversity, complexity and nonlinear dynamics encompassing many disjoints spatiotemporal scales. Despite of the informal view about complexity, which usually define a complex system roughly as a collection of many simple interacting elements showing some type of emergent and cohesive global behavior, in the general case of biological systems, the system components are not but simple and even the simplest life forms shows an astonishing complexity in many different scales. The specific case of bacterial cells can be even more complex, owing to the fact that they have short generation times and the high mutation rates, making hard to define a clear separation between intracellular and evolutionary scales as well as favoring the existence a high level of structural diversity. In front of the existing overwhelming structural diversity it is important to provide a high level functional abstraction for the cellular structures and processes in order to make the problem treatable under a computational modeling perspective. Hence, it is important to provide a comprehensive systemic and functional decomposition of a what constitute a bacterial cell under a computational modeler centric point of view. The bacterial conjugative systems, despite of being quite similar, if it is considering just a purely functional high level, presents great structural differences in the underlying molecular mechanisms between the two biggest bacterial classification groups defined by the membrane structure.

The development of mathematical or computational models, despite of the apparent simplicity which can be gathered at a first sight, once we have crossed beyond the surface border it is a painfully hard task, especially if we want to make models intended to be potentially relevant to some field or discipline and not only a mere computational toy. On the bottom line, we have to assume and interiorize the very basic truth about models, stated several times by George Box and Norman Draper [BD87] which fundamentally says that all models are wrong and some (very few) are useful. In addition, we also have to keep in mind that there is no unique mapping between any real system and the model abstraction [Min65]. Therefore, we have to be extremely careful in dealing with models of any system but this is particularly true in the case of Individual-based models of microorganisms.

The Individual-based models make easy for the practitioner to capture very com- 
plex behaviors and general ideas about the system under study without the need of being constrained a priori by any kind of formalism. Therefore, any model developed under these rules becomes a succession of state variables, random numbers and if/else statements linking all parts together. Of course, the first consequence of implementing models that way, is the complexity of the model itself which ironically in some cases emulates the complexity of the real system being modeled. Other consequence is the tendency of modelers to think about the models as if they were real entities, which should mimic perfectly the real system, forgetting that eventually all models are wrong abstractions. Additionally, the development of these kind of models, demands the mechanistic knowledge of the underlying process which, in the case of biological system, are rarely thoroughly available. Although this knowledge about all cellular activities were completely elucidated it would be computationally impractical to add so much details to a model If we are going to simulate a large population.

It is always an interesting exercise to compare the sizes of the different cellular structures in order to get a general picture from its relative importance. Therefore, taking plasmids individually each of them represents only a small fraction of total cellular DNA, ranging approximately from 1 to $200 \mathrm{kbp}$ whereas the bacterial chromosome often falls in the range of 1000 to $5000 \mathrm{kbp}$ [Fre90]. This basically means that the bacterial chromosome can be one to three orders of magnitude greater than the plasmids. Even though, the real amount of plasmid DNA inside a living cell is slightly greater than this, owing to the effect of plasmid copy number the total qualities are small when compared to the chromosomal DNA.

The occurrence of bacterial plasmids in natural environment is not an odd event, being the rule rather than the exception and it is not uncommon find wild-type cells harboring up to six different identifiable plasmid types. Most of these plasmids are normally lost within the interval of few generations [Fre90] when bacterial cells are cultured under laboratory conditions which possibly indicates that these elements are nothing but an additional metabolic load to their hosts outside of their natural ecological niches. Therefore, the elimination of these genetic elements in such conditions where nutrients are readily available and the selective pressure is absent, can be attributed to the competitive exclusion principle because uninfected cells have a slight advantage over plasmid bearing cells which, though minimal, allow plasmid free cells outcompete the infected ones. This leads, in the course of few generations, to the dominance of 
those cells not having the plasmid and that explanation holds for both conjugative and non-conjugative plasmids.

Moreover, it was recently proposed that the genetic drift can be one of the main forces behind the reduction of conjugative strength in spatially structured populations [FKJC14]. It is an interesting and unexpected result because it has always been considered that population growing under these conditions had the perfect environment for plasmid spread but it has been shown that surface-attached colony promotes the spatial separation of donor and recipient cells which in turn decreases the changes successful mating events.

\subsection{Motivation}

The bacterial conjugation, despite of the large amount of studies available which are primarily focused on the molecular and biological aspects, is still not completely understood systemically. The main cause is the lack of an integrative framework for providing a comprehensive view, qualitative explanations and quantitative predictions for the multitude of observed global dynamics. It entails the existence of multiple confronted views for the aspects related to the population dynamics hindering the convergence to global unified and homogeneous systemic view for the process. Certainly, one of the most illustrative example for the previous statement is the multiplicity of ways which distinct authors use for express the conjugation rates. There is not a single metric or standard for describing efficiency for the conjugative process, hence whereas some studies measure the conjugation as ratio between $T /(T+R), T / R$ or $T / D$, where $R$, $D$ and $T$ express the infective state and denotes respectively the number of individual cells or concentrations of uninfected recipients, donors and transconjugants ${ }^{1}$. Others used metrics are the minimum number of donor cells required for observing one conjugative event for a given time lapse and the so called $\gamma_{\text {end-point }}$ intended to eliminate the effects of other factors but more focused on continuous models [SGSL90]. Another key metric, for which there is also no consensus, is the cost of being infected, normally referred as the metabolic burden and different studies reports disparate results. That makes practically impossible to conciliate these views and compare the results which

\footnotetext{
${ }^{1}$ A previously uninfected individual which becomes a proficient donor after being infected will be able to accomplish secondary transfers
} 
consequently makes extremely complicated to unveil common patterns.

Fundamentally, that state of affairs implies a practical constraint for building an operational model of bacterial conjugation which could be useful for predicting the population dynamics based on its initial conditions and on their structural properties. The systemic knowledge of bacterial conjugation is important for several reasons but perhaps one of the most significant is the fact that the horizontal gene transfer provided by the conjugative process associated with the selective pressure is the causing of rapid spread and the settlement of multi-antibiotics resistance in bacterial populations, exceedingly reducing the arsenal available to fight against bacterial infections. Consequently, the complete knowledge of the individual intracellular aspects controlling the global dynamics would greatly help to understanding the singularities of conjugation which could lead to the generation of new strategies for talking with the bacterial infections. It would also be useful for harnessing the power of horizontal gene transfer for delivering information and making computations using bacterial cells.

The most prevalent condition for naturally occurring bacterial populations, is structured on biofilms which, despite of having a positive cost for the individuals required to produce the aggregating extracellular polymeric substances, provide physical protection and physiological benefits for the population as whole. It is normally discussed whether biofilms provide the ideal cell to cell contact conditions for mating pair formation which could facilitate the conjugative plasmids being spread through the bacterial colonies. Biofilms are not a continuous in their physiological, genetic, chemical and structural aspects. That heterogeneity is being generated as a direct consequence of spatial structure which are intrinsic to populations on solid environments. There exist divergent results about whether plasmids can fully invade or not bacterial colonies inside biofilms. It has been proposed three simple distinctive aspects related to the plasmid spread and how they are affected by the bacterial biofilms. Thus, the effect of biofilm is positive for the first transfers and the initial plasmid invasion but is detrimental for the progress of infective wave beyond the first rounds of horizontal transfers owing to the separation between proficient plasmid bearing cells and susceptible receptors. Finally, the biofilm structure has a neutral effect on the vertical transmission, which basically depends on the plasmid capacity to be faithfully maintained in their hosts across successive divisions, as well as on the metabolic cost, which is detrimental to the host fitness in absence of positive selection for plasmid bearing traits[ST16]. It is 
Chapter 1. Introduction

important to find and recognize regularities in the biological processes which are the key to localize and understand the singular and anomalous behaviors which normally leads to new discoveries.

Perhaps, one of the first approach employed for modeling the bacterial conjugation was representing the population dynamics as system of ordinary differential equations under the assumption that the process follows a mass action law dynamics [LEV79]. Roughly speaking, the law action assumes that conjugative encounters are completely random and proportional to the concentration of donor and recipient cells. These assumptions only holds true for those case where cells are cultured on liquid medium and the bacterial cells grows without spatial structure. Additionally, that approach does not provide any qualitative explanation about the intracellular processes governing the global dynamics and does not capture the individual and spatial diversity generated by the colony structure.

The first aspect which a modeler has to face when implementing a spatially explicit individual-based model to represent the bacterial conjugation dynamics on structured environments, either using a discrete or continuous space and a discrete time representation, consists that is practically impossible to achieve a smooth and good agreement between simulation output and the experimental data, relaying exclusively on the parameter describing the conjugation rate.

The population-wide or even the individual-based conjugation rates are fundamentally expressing how strong is the horizontal transfer process by accounting for the frequency or for the number of horizontal infections within some population or group of individuals. Thus, the temporal or age related information is completely ignored. This is possibly a direct consequence of the beginnings of conjugation modelling, which has borrowed some ideas of compartmentalized epidemics for representing the dynamics of horizontal gene transfer. These ideas are perfectly acceptable for the prevalent host-pathogen association, which present very different life cycles each of them. Additionally, the standard epidemic models does not have to take into account the vertical transmission component[BC93] which are important exclusively for very few pathogens such as T. cruzi., the causative agent of Chagas disease or the HIV, but the first has exclusively the vertical component of transmission. Therefore, the temporal structure can be, in most of cases, safely leaved out of the model formulation in order to avoid to increment the complexity. Nonetheless, in the specific case of modeling the bacterial 
conjugation kinetics, as we will show in this work, it is necessary to take into account not only the frequency of events but also the time when the conjugative events are most likely to happen in order to satisfactorily describe the complex dynamics generated by the intermix of horizontal and vertical spread as well as the associated fitness costs.

More recently, other approaches have been explored for building computational models of bacterial conjugation processes taking place in surface-attached colonies. One of these first approaches conceptualize the process as the encounter of two different colonies of infected and uninfected cells growing and eventually meeting each other in such way that conjugation takes place when both colonies collide [LWGP03]. Subsequently, other modeling approaches, such as interacting particle systems and cellular automata have started being employed for representing the spatial structure paving the way for more advanced methods using the agent-based modeling which have evolved as the main option for capturing the individual variability and providing a feature rich modeling paradigm for representing the bacterial cells with a great level of detail which cannot be achieved with other modeling techniques. That family of methodologies are normally known as individual-based modeling when the target of modeling activity is an ecological process [GR05a] reinforcing the fact that agents are depicting individuals from a population. The first efforts towards the spatially explicit modeling of bacterial colony growth with an individual representation of cells can be traced back to the 1998 where the BacSim [KBW98] was presented and applied for simulating the biofilm formation. Few years later, another discrete space and time simulator was introduced, the INDISIM [GLV02] focused on the vegetative aspects of bacterial colony growth. The initial version of BacSim was further updated and improved and distributed renamed as iDynoMiCS $\left[\mathrm{LMM}^{+} 11\right]$. Both simulators represent bacterial cells as spherical entities and are oriented to the simulation of biofilms. Finally, in a slightly different context we have the gro, which is more than an individual-based simulation tool, is a general-purpose tool with its own domain specific language for programming multicellular behaviors with colonies of microorganisms [JOEK12]. The gro environment have been further extended by the addition of several new features, including the support for conjugation and an improved performance reduced the time required for simulating a larger number of individual cells [GGGP+17]. The individual-based modeling efforts on bacterial conjugation process are scarce and perhaps one of the 
first successful approach taking into account the spatial structure was an interacting particle system [KLF+ ${ }^{+}$]] which has proven to produce much better results for spatially structured populations than the previous models based on differential equations.

\subsection{Objectives and Contributions}

The main objective of this work is to understanding the factors, at an individual level, governing the globally observed plasmid dispersion dynamics. Taking into account the issues previously mentioned we had developed an individual-based model for the horizontal gene transfer called BactoSIM which contains the essential standard set of cellular process representing the bacterial vegetative life-cycle, as well as, the conjugation module which have three non-overlapping alternative implementations for the plasmid transfer. We have called these alternative views, about the conjugative transfer, as strategies or model types. These tree strategies or model types, are defined by two parameters, the conjugation rate $\gamma 0$ and the cell cycle. The tree model types differs exclusively in the importance of cell cycle related parameter. The model also contains some useful outputs for understanding the balance between the horizontal and vertical transfer and the importance of each them. Therefore in this work the bacterial conjugation is fully delimited by the conjugation rate, the cell cycle linking, the conjugation cost and the conjugative machinery expression cost.

This group of four parameters has been systematically analyzed using the reference experimental data containing the population-wide conjugation rates and the values of doubling times for the different cell types allowing the identification for plausible ranges of the four parameters of our model which are better explaining and reproducing the experimental data dynamics. In order to undertake the model analysis we have used two complementary approaches, one for the parameter estimation and other for the sensitivity analysis. The parameter estimation process was carried out using evolutionary optimization metaheuristics and the sensitivity analysis was performed using the Morris screening method which allows to rank the model parameters according to their importance jointly with the Sobol variance decomposition method. From the best of our knowledge no other individual-based model of bacterial conjugation either encloses the aspects included in our model or has been subject of a complete analysis like the one presented in this work. Moreover for applying the both approaches 
we have implemented two R packages which are available on CRAN and distributed under the MIT licenses scheme. These packages are the EvoPER for applying evolutionary metaheuristics to the parameter estimation of individual-based models and the package $\mathbf{R}$ /Repast for running and analyzing individual-based model developed using the Repast framework. This work also contains another model called T4SS common pool which has been used for studding the competition of two plasmids sharing a conjugative sub-system.

We consider that the main contribution of this work for the body of knowledge related to the bacterial conjugation, is bringing to the light the importance of the cycle related parameter which have not been previously considered in other similar models. Additionally, no other conjugation model has been so thoroughly analyzed for verifying the validity of its outputs. The procedures presented here, even though widely known, have not been used together for the systematic model verification and validation, hence we believe that the work and methods described here could also serves as a guidance for other models in the assessment of their individual-based models.

\subsection{Document organization}

The current manuscript is subdivided in five parts considering the logical structure and organization of presented content. Part $\mathbf{I}$ is the introduction and contains this chapter which provides a general overview for this study presenting its main topics. Part II encompasses a brief exposition of the state of the question and includes two chapters. The chapter 2 gives a gentle overview exploring the general ideas about the bacterial cell under a functional and systemic perspective and the chapter 3 exposes the basic tenets of horizontal gene transfer and the current efforts on modeling its basic dynamics. Subsequently, part III consists in just one chapter which intends to make a clear statement about the problem addressed in this work exposing the general and specific questions which this thesis tries to answer. Part IV, entitled the proposed solutions to the research problem encompasses the chapters 5, 6, $77^{2}$ and 8 . All these chapters together constitutes the material methods and the discussion of this work showing the methodological approach and the path followed by supporting the key

\footnotetext{
${ }^{2}$ The content of the chapters 5, 6 and 7 have already been published in part or completely as separate papers which are mentioned in the 'Publications and Software' section.
} 
points of this manuscript. The chapter 5 exposes the parameter estimation process, the chapter 6 the techniques and methods employed for sensitivity analysis and finally, the chapters 7 and 8 shows the proposed model for bacterial conjugation as well as, its systematic analysis. The last one, part $\mathrm{V}$ closes this document with the conclusions and the next steps suggested by this research work. 


\section{Part II}

State of the Art 



\section{Chapter

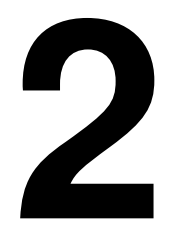

\section{Sistemic analysis of bacterial cells}

2.1 Introduction . . . . . . . . . . . . . . . . . . 17

2.2 The Bacterial Cell . . . . . . . . . . . . . . . . . . . . . . 19

2.3 The Cell Envelope . . . . . . . . . . . . . . . . . . . . . . . 21

2.4 Shape, Morphology and Dimensions . . . . . . . . . . . . . 22

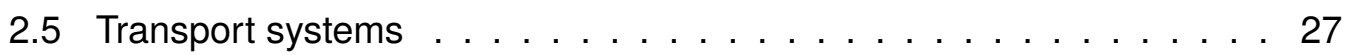

2.6 Secretion systems $\ldots \ldots \ldots \ldots \ldots$

2.7 The SOS response $\ldots \ldots \ldots \ldots \ldots$

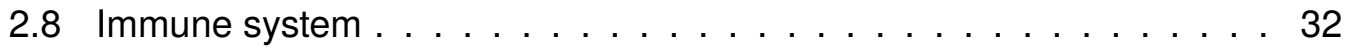

2.8 .1 Restriction Enzymes . . . . . . . . . . . . . . . . . . 32

2.8 .2 The CRISPR/Cas System . . . . . . . . . . . . . . . 33

2.9 The DNA Replication . . . . . . . . . . . . . . . . . . . 33

2.10 Energy budget $\ldots \ldots \ldots \ldots \ldots \ldots \ldots$. . . . . . . . 34

2.10 .1 The cost of conjugation $\ldots \ldots \ldots \ldots \ldots$ 
2.11 Plasmids and conjugation . . . . . . . . . . . . . . . 37

2.12 Summary . . . . . . . . . . . . . . . . . . . . . . 39 


\subsection{Introduction}

Let us not deceive ourselves, the noble art of modeling and simulation of living things is awfully complex, although when the things being modeled are tiny and supposedly simple prokaryote cells, because even the simplest form of a bacterial cell, namely the Mycoplasma genitalium which barely has 525 genes enclosed in a small chromosome of $580 \mathrm{kbp}$ or being more specific $580073 \mathrm{bp}\left[\mathrm{HCN}^{+} 16\right]$, is considerably complex, just considering the genome size as the complexity metric, which can be seen as a rough indicator for contextualizing the overall organism complexity. Of course, this is a very simplistic way for accounting for complexity because of even with that small genome the $M$. genitalium is coordinating a very large set of cascading biochemical reactions, accurately-timed across cell divisions. The Table 2.1 shows the dimensions of cell for different organisms allowing the comparison of their relative magnitudes. The individual cells are also constantly interacting in several ways with other cells in a population. Therefore, capturing every detail in a computational model for reproducing the whole internal organization of bacterial cell, although computationally feasible [KSM ${ }^{+}$12] for a simulating a single cell, may not be neither desirable nor relevant in all circumstances and must be carefully analyzed in function of the pursued objective of the simulation process. Thus, it is important to consider the modeling unit as well as the complete system under study in order to decide the what details are important and what are not relevant for the model objective, additionally the scale factor also imposes important computational constraints and what could be important for single cell studies certainly can be safely leaved out when the system being studied is a population or an ecosystem. Nonetheless, regardless of these considerations, it is worth for modelers gathering a complete view of all components of the modeling target for being able of taking a thorough and informed decision about what parts from the modeling target should be included in the model. 
Table 2.1: The comparative view for the dimensions of different cell types including viruses and bacterial strains.

\begin{tabular}{|c|c|c|c|c|c|}
\hline Organism & $\begin{array}{l}\text { Size } \\
(n m)\end{array}$ & Genes & bp & Notes & Reference \\
\hline ColE1 plasmid & & & 6,646 & & [SHPC13] \\
\hline$\lambda$ phage & 58 & 92 & 48,502 & & [Hat08, MPN16] \\
\hline T7 phage & 58 & 55 & 39,936 & & [Hat08, MPN16] \\
\hline F plasmid & & & $\sim 100,000$ & & {$\left[\mathrm{BSP}^{+}\right.$98] } \\
\hline M. genitalium & 500 & 525 & 580,073 & & {$\left[\mathrm{HCN}^{+} 16, \mathrm{LLS}^{+} 84\right]$} \\
\hline E. coli & 3000 & $\sim 4300$ & $4,639,221$ & & [BPB +97, MPN16] \\
\hline E. coli & 3000 & 5,416 & $5,594,477$ & $\begin{array}{l}\text { O157:H7 } \\
\text { strain (pato- } \\
\text { genic) }\end{array}$ & {$\left[\mathrm{HMO}^{+} 01, \mathrm{MPN} 16\right]$} \\
\hline B. subtilis & $2-5000$ & & & & [SJS +11$]$ \\
\hline T. namibiensis & 750000 & & & & [LA15] \\
\hline
\end{tabular}

Understanding what constitute a bacterial cell, from a computational modeler perspective, means initially a comprehensive functional decomposition of cell components as well the regulatory relationship linking together all of these elements. Therefore, regulatory aspects and the cell cycle coordination is also crucial for accurately representing the temporal scale of interactions between cells within a population of individual bacterial cells. It is important to capture the qualitative aspects of intracellular behavior, mainly because the quantitative data may be misleading owing to the fact that it is normally associated to very specific laboratorial conditions. In addition, only a minimal fraction of existing bacterial diversity is culturable, which means that we are completely blind to a vast portion of bacterial world. Most of the available quantitative data are taken from template organisms such Escherichia coli and Bacillus subtilis which are both by far the more studied organisms and the standard references for the gram negative and gram positive individuals respectively [JRZC14]. Once again, it is important to remark that we must be very judicious making quantitative extrapolations even for the same organism for which quantitative data was generated because the growth dynamics on culture conditions are very distinct from those observed in natural habitats. 
The next sections of this chapter are focused on individualizing the most important building blocks of bacterial cells which will be the basement for building bottom-up individual-based models.

\subsection{The Bacterial Cell}

The work of building a computational representation of a bacterial cell implies that a suitable and realistic abstraction must be provided for several the components which are part of it. Thus, the first effort towards the modeling target is getting the big picture of individual components in order to decide what must be included in the model as function of the modeling objectives. Therefore, modeling is always an activity which necessary loose information on many system variables which the main consequence is that the effects of the omitted variables are normally accounted for the variables represented in the model. That normally should not be a problem for extracting qualitative ideas from the model to the extent that model is structurally sound. In fact, modeling and building theories about physical entities are possible because of the Pareto principle can be assumed safely for the system variables, which means that a scarce $20 \%$ of system components are able to provide the explanatory power for the $80 \%$ of system trajectories. Bearing this in mind we can return to the functional decomposition of a bacterial cell. Structurally speaking, a bacterial cell, other cell types, is a container holding together the content of cytoplasmic matrix as well as the genetic material. The cytoplasmic matrix is a colloidal system containing several kinds of particles, including proteins, enzymes where, in the case of prokaryotes, all of the biochemical reactions, required for the cellular function take place.

The Figure 2.1 shows the general view of bacterial cell component tree, where can be seen the two major entities of the system: the cellular envelope and the cytoplasm. Both structures, in turn comprise a large set of subcomponents from different functional groups and properties. Thus, the cell envelope comprises the cell wall, the transport system, the different types of secretions, including the adhesins and pili systems. The cell wall determines the properties or cellular envelope such as the shape, morphology and the dimensions as well as the physical characteristics and constraints for the processes associated to these elements. The cytoplasm encompasses several components enclosed in the cytoplasmic matrix and, additionally the functionalities associ- 


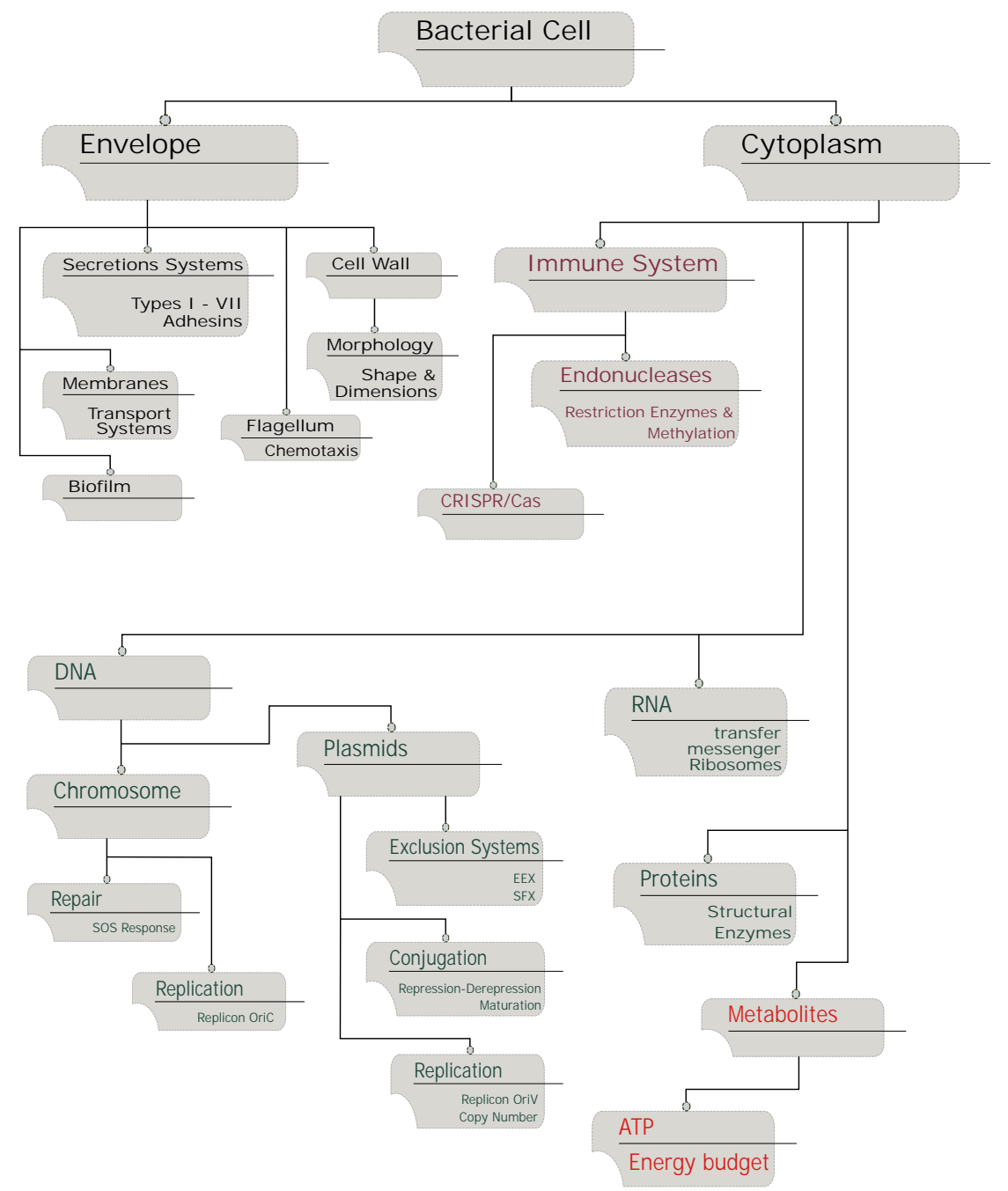

Quorum Sensing Transduction Transformation

Figure 2.1: The schematic view of bacterial components. The figure shows a simple functional decomposition for the most important cellular sub-systems.

ated with these elements. Thus, among other constituents, the cytoplasm contains the proteins, ions, the chromosomic DNA, some variable amount of plasmid DNA, several kinds of RNAs including the ribosomal DNA, different types of proteins including those catalytically active such as enzymes, the ATP and the related cellular energy budget. 
In addition, functionally the cytoplasm contains the modules required for the functions related to the DNA replication and repair subsystems, the cellular division, the plasmid maintenance and conjugative systems which their associated exclusion systems and the pheromone-driven inducer which is the hallmark of gram positive conjugation. It is somewhat artificial, as any attempt to impose boundaries for classification purposes, but necessary for a systematic approach to the bacterial cell modelling. For instance, we have placed the entry exclusion systems (eex) on the cytoplasm because its coded on the plasmids structural unit but they are actually active on the cell wall. Nonetheless we hope that this should not interfere with the main objective of this overview.

\subsection{The Cell Envelope}

The cell envelope is the membrane and its associated transmembrane structures playing the major structural role separating the external environment from cytoplasm and holding together the cell content as well as providing the required interactions with the exterior through the membrane associated transport systems allowing the uptake of nutrients and the elimination of sub-products generated by the metabolic activity[SKW10]. The structural differences in bacterial envelope has settled the basis of first classification system for telling apart the bacterial cells in two large groups, namely the gram-negative and gram-positive [SHPC13], hereafter referred as $G$ - and $G+$. Thus, once bacterial cells have been dyed using the Gram staining procedure, the $G+$ and $G$ - will assumes a deep blue and pink color respectively. The structural difference between these two groups is fundamentally that the outer membrane, which is the hallmark of $G$ - strains, is not present on gram positive bacterial cells, in addition the internal peptidoglycan membrane is much thicker in gram-positive bacterial cells[Koc90]. A notable exception for that rule can be found in those bacteria from genus Mycoplasma which, despite of being genetically related to gram-positive cells do not have the distinctive large peptidoglycan wall which instead, in their place have the cell membrane made of sterols [Hog13]. The outer membrane structure is made of lipopolysaccharides (LPS) which is a type of glycolipid known for triggering septic response [SKW10]. The distinctive composition of bacterial envelopes of gram-negative and gram-positive strains raise a subtle difference in the conjugative systems associ- 
ated to these two groups which in the case of $G+$ seems to be modulated due to the production and extracellular deployment of pheromone like elements by the plasmid free cells, in the extent that these molecules are deemed to induce plasmid bearing cells to accomplish the conjugative transfer. More specifically, the pheromone-like systems are comprised by hydrophobic polypeptides of approximately eight amino acids expressed by recipient cells whose the main identified effect is inducing the expression of adhesins in the cell envelope and subsequent the formation cell groups facilitating the close contact between donor and recipient cells which is a precondition for conjugative transfer[Sum96].

On the other hand, no evidence of a similar mechanism has been observed for gram-negative strains but the existence of exclusion systems can play a role functionally similar to the pheromone-induced conjugation in the sense that no conjugative transfers are observed to an already infected individual[GBdlC08] which would constitute evolutionarily not justifiable energy expenditure. Therefore, from the modeler point of view, the same modeling pattern can be safely applied for both systems, consisting in having for each agent and state variable for expressing the plasmid presence and the primitive has_plasmid $(\mathcal{A}, \mathcal{P})$ where $\mathcal{A}$ is an agent instance and $\mathcal{P}$ the plasmid type. The primitive will return a Boolean true if agent $\mathcal{A}$ is already infected and false otherwise.

\subsection{Shape, Morphology and Dimensions}

The prokaryotic cells are quite diverse in aspects related to their morphology and dimensions, where the smallest cells, belonging to the genus mycoplasma, have sizes of between 0.15 to $0.3 \mu \mathrm{m}$ and the largest bacteria identified to the date, the Thiomargarita namibiensis has a diameter of approximately $750 \mu \mathrm{m}$, in other words, the difference between the smallest and the biggest one is spanning four orders of magnitude [LA15]. With respect to their shape, bacterial cells can normally found in forms as rod-shaped, spherical, curved like a comma or spiraled which are respectively known as bacillus, coccus, vibrio and finally spirochaete Hog13], additionally the helix-shaped bacteria like Helicobacter pylori[SRP+13] must also be mentioned. The bacterial morphology and its dimensions are directly related to the habitat and the ecological niche occupied by each strain. For instance, the helix shape of $H$. pylori is an adaptation which facilitates 
the cell movement and colonization of gastric mucus[SRP+13]. The different bacterial morphologies show distinct behaviors with respect to the disposition of daughter cells generated after division. For instance, the rod-shaped cells, as general rule, do not form groups and divides on the same reference plane making the colony structure and disposition a consequence of forces generated by the cells pushing each other during their growth. On the other hand, coccal forms shows the tendency of dividing along to several reference planes forming collective of cells which are known as diplococcus, staphylococcus, streptococcus or sarcina.

The bacterial shape represents an important adaptive aspect of bacterial cells allowing them to tackle with selective forces, such as the competition for nutrient access or colonizing a wide range of habitats which may require the ability of individuals for moving toward a chemical gradient or attaching to surfaces [You07]. Despite of existing diversity, the two prevalent most prevalent bacterial morphologies are the bacillus and the coccus shape. The rod-shaped morphology seems to be controlled or at least partially determined by the $M r e B$ protein bundle as has been proposed in a studies [BFW ${ }^{+}$09] [JSMS11] carried out with E. coli cells where the addition of $A 22^{1}$ to the growth medium disrupts their he normal morphology making the cells to assume a spherical shape. Apparently, the A22 molecule depolymerizes the MreB protein. The same effect has also been observed [JSMS11] in another G- bacteria, the Caulobacter crescentus which is also an important model organism for bacterial cellular studies. The $M r e B$ protein contributes to the cell wall strength and supposedly acts adding mechanical rigidity to the peptidoglycan mesh [JSMS11].

From the modeler perspective, it is important considering the envelope dimensions, the volume which is conditioning the cytoplasmic density for capturing realistically the aspects related to the nutrient uptake as well as the cellular elongation. The endocytosis rate depends on the cell wall contact surface with and extracellular elements nutrient sources and, the total contact area in turns is function of bacterial shape. The envelope structure of cocci shaped bacteria can safely be approximated, for the modeling purposes, as a sphere which means the cellular volume is given by the simple expression $V=4 / 3 \pi r^{3}$ and the contact surface as $S=4 \pi r^{2}$. The bacillus, in turn requires the summation of a cylinder and a sphere, as shown in Figure 2.2 for approximating the real volume of a rod-shaped cell. Thence, the surface can be calculated

\footnotetext{
${ }^{1}$ S-(3, 4-dichlorobenzyl) isothiourea
} 
as $A=2 \pi r^{2}+2 \pi r h+4 \pi r^{2}$ and volume using the simple expression in the Equation (2.1),

$$
V=\pi r^{2} h+\frac{4}{3} \pi r^{3}
$$

the terms $r$ and $h$ denotes the radius and the cylinder height as usual.

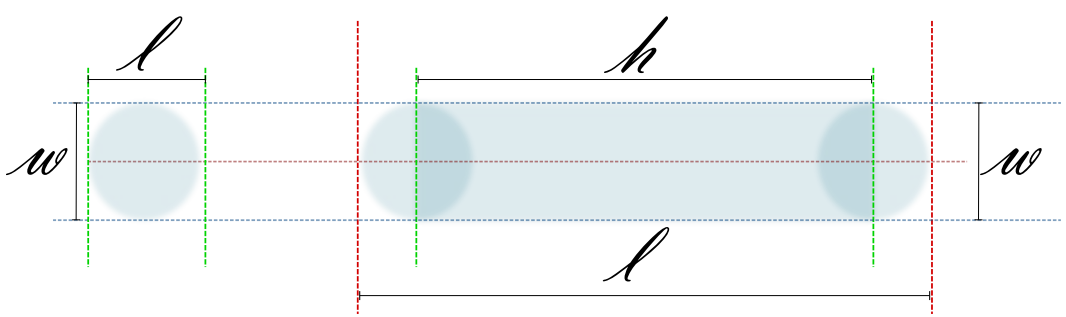

Figure 2.2: The bacterial envelope volume approximation. The shape on the right is the approximation with cylinder plus a sphere, for the volume of a bacillus cells. The shape on the left side represents the coccus morphology which is an special case where $l=w$.

That simplistic theoretical approximation can be contrasted to the results obtained from experimental studies using image analysis techniques to densiometric and transmission electron microscopy data [LKKP98] which provides an estimator for the cellular volume of a Escherichia coli strain given by the Equation (2.2),

$$
V=\left[\left(w^{2} \frac{\pi}{4}(l-w)\right]+\left(\pi \frac{w^{3}}{6}\right)\right.
$$

where $w$ denotes the width or diameter and $l$ is the length of rod-shaped cell in its longitudinal axis. It is interesting to note that formula can also applicable to coccishaped cells when the values of $l=w$ making the term $(l-w)$ equal to zero. The same principle can also be applied to the estimation of surface area which is given by the expression $A=\pi w(l-w)+\pi w^{2}$. Another remarkable aspect of cell envelope, is that despite of size and volume variations along the cell cycle, the cytoplasm density $\rho$ is kept practically constant from the cell birth to time of division [CCS01]. The main consequence of conserved densities, assuming a computational model evolving 
in discrete time steps, is basically that $\rho_{t} \equiv \rho_{t+1} \equiv \ldots \rho_{n}$, being $n$ the total number of simulation steps. Therefore, when the model incorporates the state variables describing the cell mass $m$ and the cellular volume $v$ the constraint shown in the Equation (2.3),

$$
\frac{m_{t}}{v_{t}} \simeq \frac{m_{t+1}}{v_{t+1}}
$$

which can be rewritten, arranging the terms, as $v_{t+1} \simeq\left(m_{t+1} v_{t}\right) / m_{t}$. Thus, the update scheme for the state variables must enforce that the constraint hold for keeping the simulated cellular dynamics consistent with the real cellular behavior during the growth which also delimits the nutrient uptake process. The same modelling pattern can be applied for updating the cell length state variable, which is depicted in the Equation (2.4),

$$
f\left(m_{t}, m_{t+1}, \text { length } h_{t}\right)=l_{t+1}=\frac{w_{t+1}{ }^{3} m_{t+1}-w_{t}^{3} m_{t+1}+3 w_{t}{ }^{2} l_{t} m_{t+1}}{3 m_{t} w_{t+1}{ }^{2}},
$$

which links the new cell length $l_{t+1}$ on the next simulation time step with the increase on the cell mass $\Delta_{m}$. The terms $m_{t}$ and $m_{t+1}$ are respectively the current value of cell mass state variable and the updated value for the next iteration. The increment on the cell mass is the outcome of the uptake process. Finally, the terms $w_{t}$ and $w_{t+1}$ represent the cell width which can be considered constant for rod shaped cells along their cell cycle. The factors governing the size and shape diversity for bacterial cells are not completely understood [You04] and it is normally assumed a bias towards small dimensions mainly owing to the biophysical constraints related to the surface to volume ratio required to keep the cellular biochemical machinery running [You07]. By using these very simple math tools, a modeling pattern for envelope growth and elongation can be defined as shown on Figure 2.1, serving as a reusable component for individual-based models which requires the representation of elongation dynamics. The pattern can also provide answers for questions like why rod-shaped bacteria can be bigger than spherical ones or how individual bacterial cells grows.

The application of envelope growth and elongation modeling pattern requires the experimental elucidation for the parameters defined the cell envelope, namely the cy- 


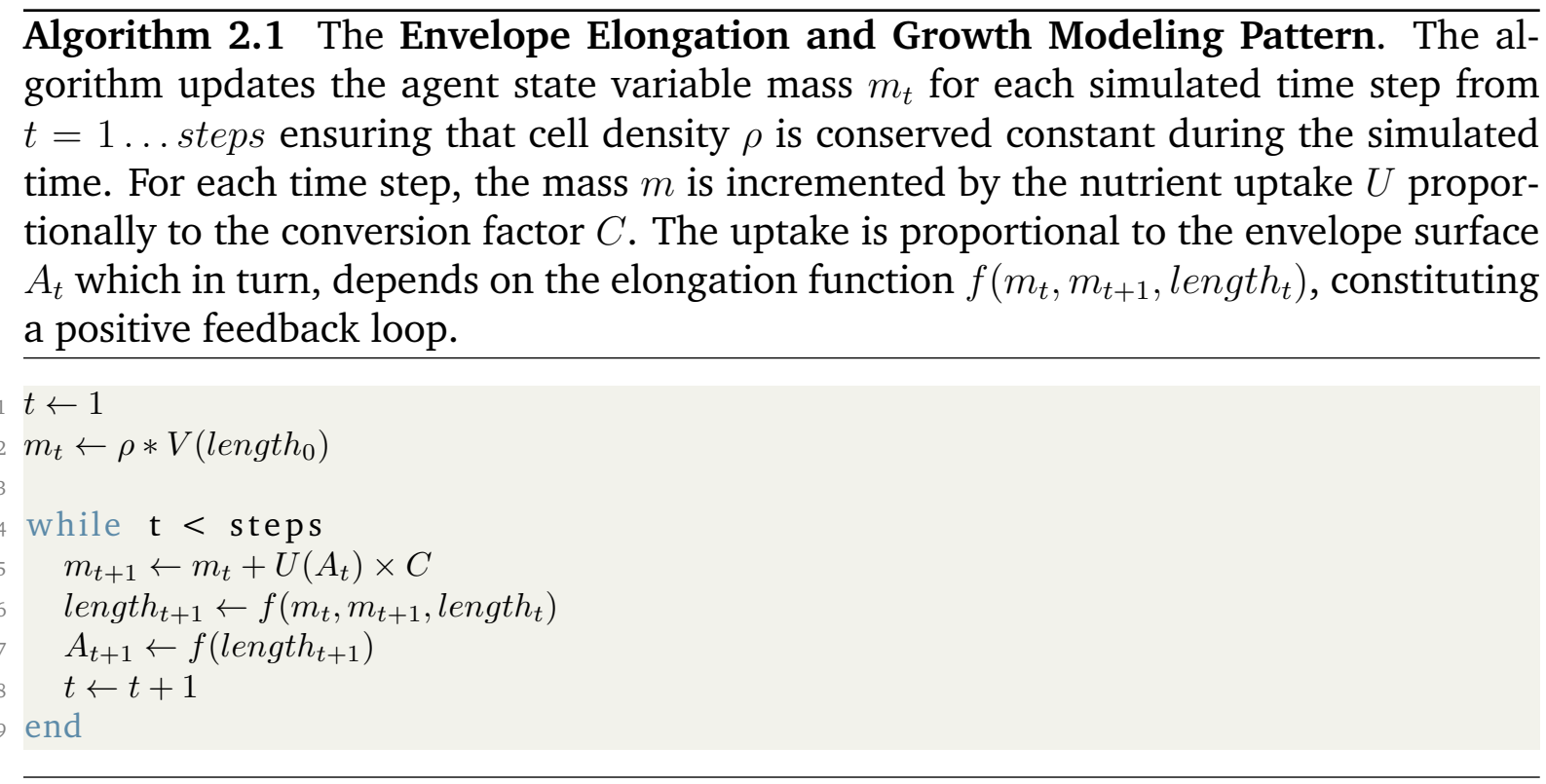

toplasm density $\rho$, the cell mass, the cellular volume and the length and width of cell capsule which is a rather complicated task even for the Escherichia coli by far the most studied, cultured and the reference prokaryote organism. The amount of studies measuring these essential cell data are rather scarce as can be seen on able 2.2 which summarize the reference values for envelope parameters. The values provided should be taken with caution because there is a great variability in cell parameters which are largely due to the varying growth conditions but in any case, the upper and lower bonds can be an acceptable reference for modeling purposes. 
Table 2.2: The parameters for the envelope elongation and growth modeling pattern.

\begin{tabular}{|c|c|c|c|c|}
\hline Parameter & Value & Unit & Notes & Reference \\
\hline mass & 665 & femtograms & $\begin{array}{l}\text { The total mass of Escherichia coli } \\
\text { O157:H7 strain. }\end{array}$ & [AIC08] \\
\hline mass & 83-1.172 & femtograms & $\begin{array}{l}\text { The value refers to the dry mass } \\
\text { of an E. coli DSM } 613 \text {. }\end{array}$ & [LKKP98] \\
\hline mass & $3-1.177$ & femtograms & $\begin{array}{l}\text { Samples from natural population } \\
\text { taken from two lakes in Austria. }\end{array}$ & [LKKP98] \\
\hline mass & $\approx 1000$ & femtograms & $\begin{array}{l}\text { Not from experimental data, it is } \\
\text { just an approximate. }\end{array}$ & [PKTG12] \\
\hline$\rho$ & 1.1 & $\mathrm{~g} \mathrm{~cm}^{-3}$ & Secondary reference. & [LKKP98] \\
\hline
\end{tabular}

The Figure 2.3 shows a simple simulation of the bacterial envelope parameters for spherical and for rod-shaped cells. As can be observed, the rod-shaped cells present a significant advantage over the spherical ones as they can grow keeping a quasilinear surface-to-volume ratio and consequently maintaining the required uptake rate. The underlying assumption is that the during their growth the bacterial cells maintain a practically constant density which in turn require a constantly increment in the uptake rate for spherical cells which cannot be achieve as the surface to volume ratio quickly decreases making inviable that these type of cells shows large sizes. On the other hand, rod-shaped cells can maintain linear growth regime and do not have that restrictions.

\subsection{Transport systems}

The term transport systems, in the context of bacterial physiology and the envelope study, stands for those mechanisms which are used for carrying nutrient particles from the substrates available on environment surrounding the bacterial cell to the interior of cell where it will serve as raw materials for the catabolic and anabolic reactions required for all cellular activities and housekeeping. From the modeler perspective, 

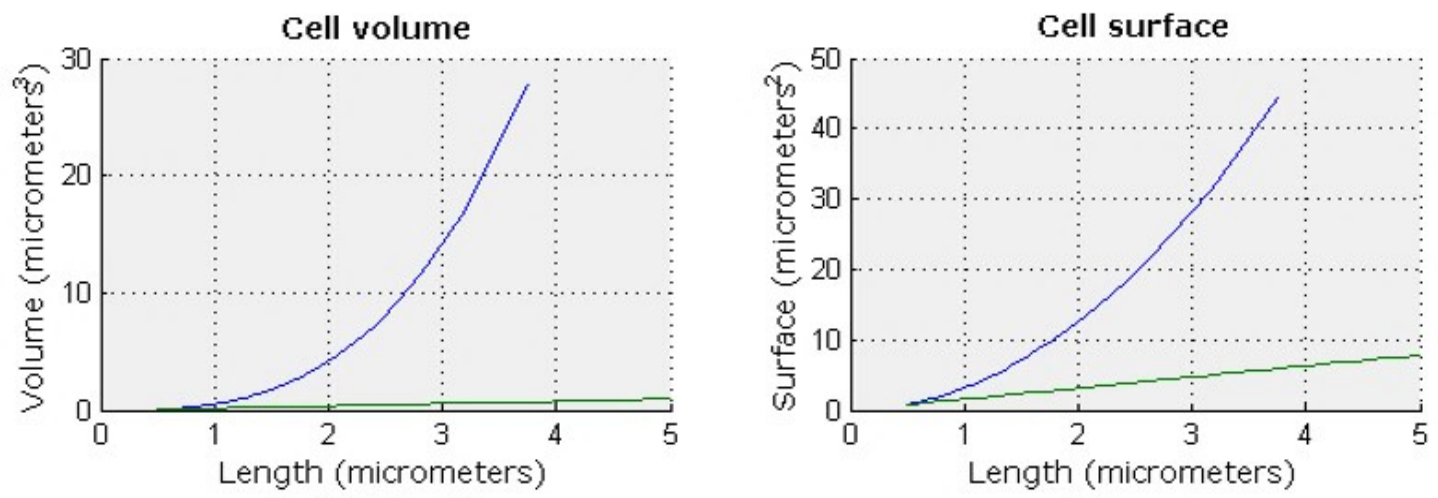

Cell S/V ratio
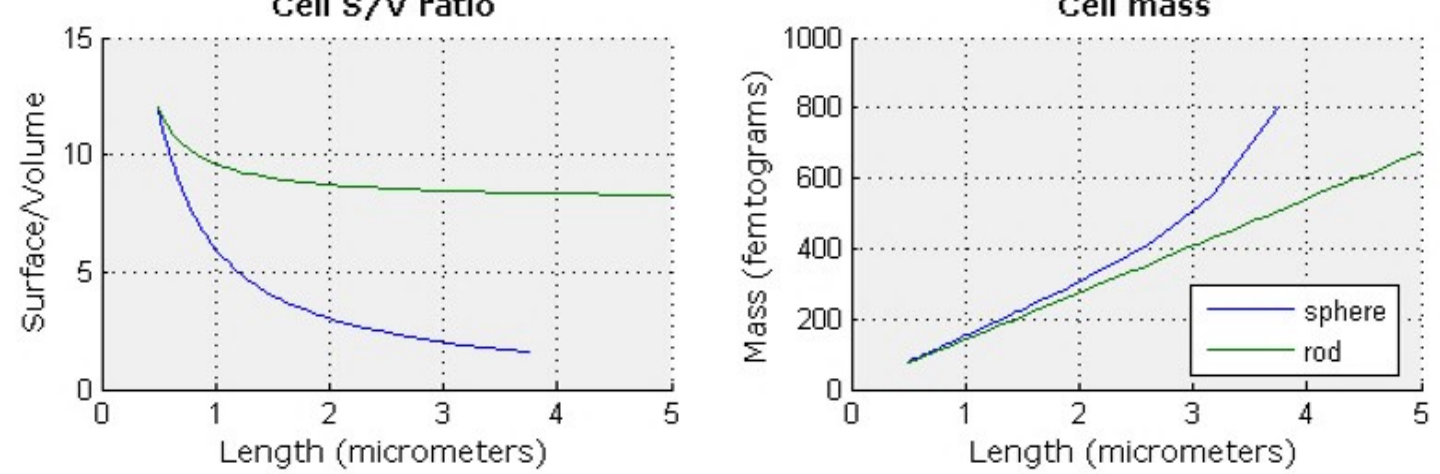

Figure 2.3: The effect of bacterial cell shape on the envelope-related parameters.

the transport systems and their characteristics can be important for being considered in the model implementations of the bacterial nutrient uptake system when a more accurate dynamic of bacterial growth is required. The overall bacterial growth regime essentially depends on the concentration of nutrients outside of cell as well as on the efficiency of mechanisms available for transferring these nutrients from the outside to the cytoplasm overcoming a concentration gradient which implies that the associated processes required the allocation of resources in the form of energy expenditure. Thus, the global rate of growth of bacterial cell is functionally dependent on the uptake rate which, in turn depends on the available transport mechanisms. The nutrient uptake is in some extent conditioned by the structural properties of cellular envelope despite of the fact that the transport system is situated in the cytoplasmic membrane which in turn is surrounded by the cell wall and the outer membrane in the case of gram negative bacteria. 

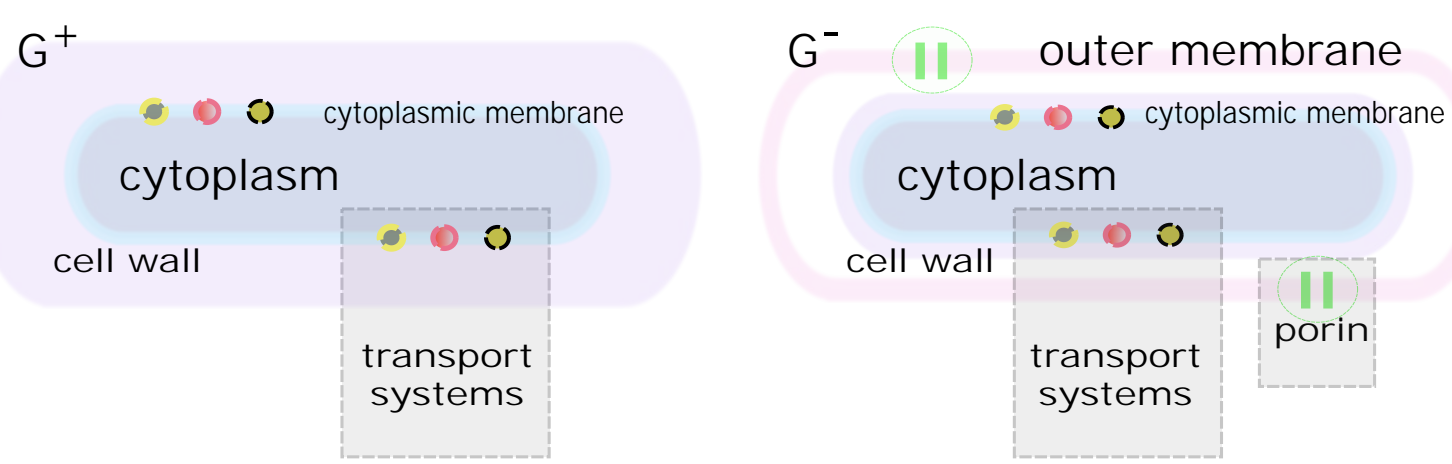

Figure 2.4: The transport systems.

\subsection{Secretion systems}

The ability of carrying the produced proteins on the interior of cells, from the cytoplasmic matrix to either the surrounding environment or to the interior of other cell types, including eukaryotes is a common and widespread conserved feature of many eubacteria and archaea[ASD06] strains which is known collectively as secretion systems. There are seven types of secretion systems identified and described each of them serving for an specific purpose for transporting a determinate kind of product[GM16].

The secretion systems, specifically the T4SS is of central importance for conjugative systems found on gram negative bacteria. The most well characterized system is found on the plasmids of incompatibility group $F(i n c F)$, represented typically the derivatives of $\mathrm{F}$ factor of $E$. coli. The genes of transfer region of the $\mathrm{F}$ plasmid, known as tra is considered as subset of T4SS family and contains eight out of ten of its core genes[LKGF03a].

\subsection{The SOS response}

The practical totality of living cells, as well as viruses, are provided, by the evolutionary forces, with several mechanisms for tackling with the task of ensuring that their chromosome is faithfully replicated and segregated to the daughter cells across generations. The bacterial chromosomal DNA content can suffer some kind of damage triggered by many environmental factors, such as ionizing radiation, UV light, chemicals and so on which due to the short generation times can quickly become catastrophic for a popula- 
Chapter 2. Sistemic analysis of bacterial cells

tion as the sequences are replicated much faster than in other types of cells. Therefore, bacterial cells can adopt distinct strategies for correcting the damaged DNA sequences. The $E$. coli has many different mechanisms [SHPC13] implementing pathways for recovering distinct types of DNA damage, namely the Methyl-directed mismatched repair, the Very short repair, the guanine oxidations (GO), the Alkyl, the Nucleotide excision, the Base excision, the Photoreactivation, the Recombination repair and finally the SOS response subsystem.

Among all of these previously mentioned repairing systems, the SOS response should be take into account explicitly or implicitly for the purposes of modeling the conjugative horizontal gene transfer process. During the conjugation process, as single strand of the plasmid DNA is transferred from the donor to the recipient cell which induces the activation of the SOS response system [BBM10]. The level of complexity of SOS response is high and the complete system includes a minimum of 25 genes. The overall target of SOS response is to overcome the effects generated by the formation of pyrimidine dimers due to the cell exposition to ultraviolet light or to the ionizing radiation breaking apart the phosphodiester bonds. Both situations prevent the completion of the transcription and the replication processes which are recovered by the SOS system [Pie04] with the side effects is the introduction of replication mistakes on the new synthetized DNA strand.

It is interesting to note that between the different corrective actions implemented by SOS response system for recovering damaged DNA is the blocking of the division process in order to avoid that the daughter cells being produced without faithfully inheriting a copy of parent chromosome. The complete bacterial cytokinesis, that is to say, the septum formation, the fission and the ultimate cell division depends on several gene products but certainly the main role is occupied by the filamentation temperaturesensitive protein $Z$ the FtsZ protein for short [dB16]. This protein in conjunction with the FtsA are the main elements of the Z-ring essential for cytokinesis [Du 13] in such extent that inferring with the FtsZ protein leads to the disruption of bacterial cell division.

The Figure 2.5 shows an overview about how the SOS response stops the normal sequence required for cell division. The subsystem is triggered by the accumulation of single stranded DNA (ssDNA) beyond the normal levels which cause the activation of RecA protein as the single ssDNA is the substrate for its polymerization [Fre90] [Pie04] [SHPC13]. Subsequently, the RecA/ssDNA leads to the cleavage of the LexA 


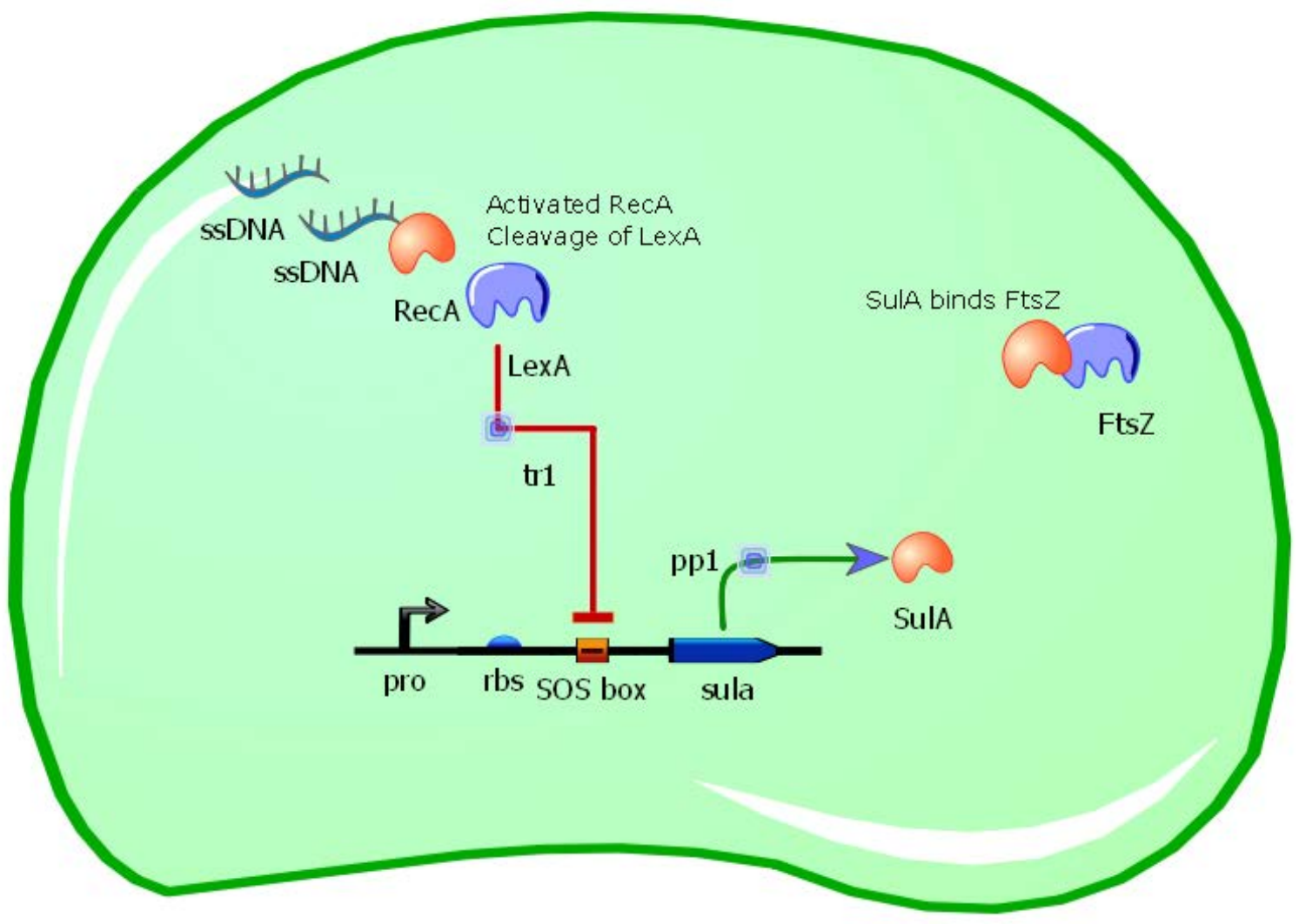

Figure 2.5: The schematics view of SOS response regulatory network.

repressor which is negatively regulating the expression of the sulA gene. The protein SulA suppresses the division by bind the FtsZ protein, hence preventing the Z-ring formation delaying the division until the damaged DNA or ssDNA returns the reference values.

During the conjugation, the plasmids are transferred as ssDNA which implies the activation of the receptor cell SOS system and consequently making these cells to have a cell cycle comparatively larger than the other transconjugant cells which have already added the complementary strand and circularized the plasmids. This affect the global dynamics as a poor fitness values for the transconjugant cells which limits in some extend the plasmid spread[Sum96]. Some plasmids, such as the R64drd, RIP113 and the R100-1, have evolved for circumvent this limitation and have a mechanism codded by the plasmidic SOS inhibition, the psiB gene which interferes with the host SOS 
system, although not all plasmids, which contains this gene, are unable to express it with the required levels for being effective[BBM10].

\subsection{Immune system}

The bacterial immune system, despite of the structural differences, has the virtually the same functional role as the immune system found in the higher animals but obviously has a much simpler implementation. These functions are basically the ability of recognizing what constitute self and the non-self elements and, once identified the foreign element, to attack and destroy them [GH06]. The vicissitudes of bacterial way of life expose them to many different threats which could disrupt or be detrimental for cellular activities. In their natural environment, bacteria are subject to the attack of phages as well as being infected by plasmids, even though the last one pose a potential adaptive selective advantage when the bacterial cells are exposed to antibiotics, most of the times are just a metabolic load. Additionally, any type of foreign DNA could be potentially disruptive for the legitimate cell metabolic activities. For tacking with these threats the bacterial cells have evolved some not so rudimentary immune defenses [SHPC13].

\subsubsection{Restriction Enzymes}

The first mechanism identified in bacterial cells for protecting them against the foreign DNA was the restriction-modification system. It was discovered as consequence of the detection that the $\lambda$ phage was 10,000-fold less efficient infecting the of it E. coli K-12 strain than the B strain [Fre90]. It is interesting to note that not all bacterial strains are equipped with this immune system. The functional description of this system is very straightforward, that consists in a enzyme, known as restriction endonuclease which is able of cutting an specific sequence of DNA but at the same time modifying the recognition sequence of the bacterial DNA by the addition of a methyl group to a cytosine or adenine which effectively blocks the action of the restriction enzyme. With this simple approach, the foreign DNA is cleaved and subsequently degraded [Fre90]. Nowadays, approximately 4000 restriction endonucleases have been identified and catalogued [RVPM15] each of them being able to recognized and cleave a specific DNA se- 
quence. There are basically three different implementations of restriction-modification systems which differs amongst other things in the ATP requirements, the dimension of the recognition sites and on how the double stranded DNA is cut [Pie04].

\subsubsection{The CRISPR/Cas System}

Whereas the previous restriction-modification system is a sort of statically defined immune system, the CRISPR/Cas ${ }^{2}$ system is a true adaptive immune system being able to emulate, in some extent, the acquired immunity with is the hallmark of the immune system of high organism. Additionally, it is inherited and transmitted across successive generations. The acronym CRISPR stands for clustered regularly interspaced short palindromic repeats which is a clear description of its structure. The CRISPR system is present in approximately $90 \%$ of archaea and $40 \%$ of bacteria[HB10]. Structurally speaking, the CRISPR consists in many direct repeats, which are normally noncontiguous separated by what is termed as spacers. The spacers are high variable sequences because they are taken form the invading DNA sequences of plasmids and phages. Usually, both components have a length of few base pairs, being the repeats within a range of 23-47 base pairs and the spacers a size of 21 to 72 base pairs[HB10]. The CRISPR associated proteins (Cas) encloses functionalities such as nucleases, polymerases and helicases.

\subsection{The DNA Replication}

The interdivision time is not homogeneous and can show much variability between individuals of the same bacterial strain. While in the $\mathrm{E}$. coli B/r strain the bacterial cells tend to divide practically in the same period, other strains such as $\mathrm{K} 12$ and B presents a high individual variability having a coefficient of variation ranging from 10-20\% [Fre90].

Based mostly on gram-negative bacterium E. Coli studies, it has been assumed that the growth dependent accumulation of DnaA protein instantiates the chromosome replication. It has been observed in Bacillus subtilis that the initiation time of chromosome replication is unaltered in cells that are approximately $30 \%$ small than wild-type,

\footnotetext{
${ }^{2}$ The term Cas stands for CRISPR associated protein which are together the ground of prokaryotic acquired immunity
} 
Chapter 2. Sistemic analysis of bacterial cells

contradicting the idea of growth dependence. It also has been found that changes in DNA replication affects the cell size in both types of bacteria. In the Escherichia coli cells the absolute amount of DnaA which progressively accumulates along the cell cycle seems to be the trigger of DNA replication instead of its cytoplasmic concentration.

\subsection{Energy budget}

It is difficult to find a single and conclusive view about the real metabolic burden imposed by any plasmid to their hosts. While some authors consider the cost as being as low as $1 \%$ others are showing figures as high as $30 \%$ of penalization. The reason for these discrepancies certainly lies on the fact that it is not being measured the same thing and failing to taking into account the distinct phases of plasmid infection life-cycle. Additionally, most measurements of host fitness are taken for the whole population level leading the wrong estimation of individual-level values.

A much better approach would be to understand the metabolic cost as a function of some structural property of the plasmid-host association and generate a simple theoretical framework which could be proved or refuted by the observations and not the opposite. Making the assumptions of a simple linear relationship between replicative energy budget and DNA stretch size and also considering that bacterial cell is in a steady state, being the only energetic expenditure for the plasmid the required for replicating it, the metabolic cost can be expressed as $C=L(P) / L(C)$, where $\mathrm{C}$ is the metabolic cost expressed as the ratio between the plasmid and the chromosome size. Therefore, the cells infected may have their cycles increased by that estimated cost. For instance, a rough estimate for the minimal metabolic cost required for the maintenance of an F plasmid in an E. coli cell can be calculated simply on the basis of plasmid size. The size of E. coli chromosome is approximately $4500 \mathrm{~kb}$ long and the average size of $\mathrm{F}$ plasmid is close to the $95 \mathrm{~kb}$ [Pie12] [Fre90] which give us a cost $C=95 / 45000.02$ or what is the same thing, the cell cycle of infected cells would be possibly a $2 \%$ larger than the plasmid free cells. Of course, this is an illustrative simplification indeed, actually the things are slightly more complex but even so it is a reasonable approximation for one type of cost imposed by a plasmid.

Naturally, the previous explanation is a simplistic approximation to just one facet of the problem of determining the plasmid cost. Recently, a more systematic classification 
of the root causes of metabolic burden imposed by harboring non-essential foreign DNA, which can be assigned to six cost groups taking into account the underlying mechanism responsible for the fitness reduction [ $\left.\mathrm{BSK}^{+} 13\right]$. These mechanisms are fundamentally those derived from Energetic cost, the changes in phenotypic traits, the crosstalk in regulatory networks, the cytotoxicity, the sequestration of cellular resources and finally other system-level effects.

The energetic cost of foreign DNA refers to the fact that the plasmids, as autonomous replicons, need to drain some part of the basal energy budget of host cell for their housekeeping activities, such as recircularization, replication and copy number maintenance as well as for the expression of plasmid genes. The energy required for plasmid maintenance is not generally to high[HB12][LM15] and the most significant part of energy budget can be clearly attributed to the gene expression, especially to those related to the transmembrane proteins required for the conjugative machinery.

Another cause of fitness reduction can be assigned to the disruptive changes in the phenotypic traits which can occur when the foreign DNA is incorporated by recombination to the main chromosome which can somewhat produce the modification of the expression of one or more genes responsible for important phenotypic traits. Every bacterial cell during have complex regulatory networks controlling and coordinating all cellular processes and these regulatory networks can be altered by the products of foreign DNA regulatory system which is known as crosstalk in regulatory networks. The sequestration of cellular resources stands for the plasmids interference on cellular machinery by using actively the ribosome resources for their required genes. The interference arises when chromosome and plasmid genes are requiring a ribosome active site for being translated which increases the host cell cycle. It has been pointed out that plasmid foreign DNA is more susceptible to failures in folding process of its protein products. In high concentrations, misfolded proteins show an elevated cytotoxicity effect which may interfere with cellular processes. Finally, it is also mentioned in [ $\overline{\mathrm{BSK}^{+}}$13] the system-level effects are those related to complex systemic interactions which are triggered downstream of a primary change. 


\subsubsection{The cost of conjugation}

The global effect of conjugation and the plasmid infection on their hosts is an increment in the duration of cellular cycle meaning an overall reduction in the nominal growth rate. This is known generically as the plasmid metabolic burden or the plasmid penalization. The knowledge about the process is scarce as well as de common values for this metabolic penalty which range from values little as one percent up to more than $30 \%$ of increment in the doubling time of infected cells[ $\left.\mathrm{dCRC}^{+} 12\right]$. These figures are taken from population wide measurements and it is surely hard to use it for individual-based models. There is also no suitable explanatory theory for explaining the effect of plasmid infection on their hosts and the experimental studies fail to provide useful data for constructing a bottom-up theory of conjugation cost.

In order to circumvent that limitation, we propose a simple mechanistic approach for understanding the cost of plasmid infection. Thus the metabolic burden of conjugative process expressed as a cost function is summarized the Equation (2.5)

$$
\phi=P_{r}+P_{c n}+P_{\text {genes }}
$$

where $r$ is the plasmid replication cost, $c$ the cost of maintenance of plasmid copy number and $g$ the cost of genes which are being expressed by the plasmid during the cell life cycle. The energy driven cellular process are carried out hydrolyzing ATP molecules which could be seen as the currency for our cost system. The number of ATP required for the most of plasmid processes can be determined based on the plasmid structural properties. Hence, on average approximately 12 ATP are required for replicating every base pair [Voe] [Str], of course it is a rough approximation and the actual value depends on the kind of nucleotide is being replicated. The overall fitness reduction should not be confused with the metabolic penalization because it is consequence of multiple processes interfering with the normal cell functions, such as the SOS response as we have seen previously. Therefore, the two concepts should be reverently treated as separated entities from the modeler perspective. 


\subsection{Plasmids and conjugation}

The Domain Bacteria encompass one of most successful, diverse and abundant form of life on earth. Part of this diversity is certainly a direct consequence of factors such as a succinct genome, short generation times, modulated mutation rates which are complemented by the existence of a feature rich supra-individual gene pool which is readily available for individuals in a population through different mechanisms. One of these mechanisms is the bacterial conjugation which is basically a form of horizontal gene transfer where cluster of genes is transferred from cell to cell within populations. The plasmids, which are the fundamental unit of horizontal gene transfer, are circular double stranded DNA and they are also autonomous replicons replicating independently of bacterial chromosome and having their own life-cycle.

Plasmids can also cross the domain boundaries and infect eukaryotic cells, as can be observed in genus Agrobacterium, responsible for causing plant diseases. That is the case of $A$. rhizogenes and A. tumefaciens and their associated plasmids which are termed $\mathrm{Ri}$ and $\mathrm{Ti}$ standing respectively for root inducer and tumor inducer plasmids. These plasmids are responsible for hairy root and crown gall plant diseases respectively[DVS83][Far93]. On the other hand, the genus Rhizobium and its associated plasmids induce the nodule formation on plant roots acting as symbionts in the atmospheric nitrogen fixation process[CHGS17]. These plasmids can also be harnessed for the insertion of T-DNA in plants to create transgenic cultivars. The bacterial gene pool is also used for genetic engineered plant cultivar creation such as herbicide resistant transgenic plants[Nes15]. That is the case of aroA gene coding the AprA enzymes which makes the plant show tolerance to glyphosate[ $\left.\mathrm{RBH}^{+} 83\right]\left[\mathrm{HGB}^{+} 02\right]$. Last but not least important, plasmids are deemed to be the main cause of spreading the multi-drug resistance associated with bacterial populations exposed to the antibiotic selective pressure. In fact, this severely limits the arsenal of drugs available to fight against bacterial infections.

Conjugative plasmids are beginning to be seen as a viable tool as the wiring protocol for population wide computations, as the basic bricks for bacterial nano-networks and for more complex applications in synthetic biology but despite of the high relevance, there are either no dependable technique readily available yet, which have been thoroughly tested and systematically validated against the experimental data or 
simply an accepted standard to model the plasmid spread dynamics using single cell resolution. There is also more open question than answers on many points of the lateral gene transfer process with some opposing views about some specific aspects.

The conjugation is a form of horizontal gene transfer where conjugative plasmids are transferred from cell to cell in a bacterial colony. Conjugative plasmids are double stranded DNA which replicate independently from bacterial main chromosome, containing also the required genes to express the conjugative machinery, including the conjugative pili, making possible the ssDNA transfer across cellular envelopes. Despite of the greatest focus on plasmid as a tool for synthetic biology and the wealth of molecular studies available little is known about the inner systemic structure of the conjugative process itself and how rules for modeling can be defined on a structurally realistic basis.

The bacterial conjugation is a natural process where bacterial cells interchange circular DNA fragments known as plasmids. Conjugative plasmids are the vehicles of antibiotic resistance spreading in hospitals, and additionally may carry other genes which are responsible for bacterial virulence and pathogenicity [NHS09]. Therefore, it is worth to understand how they are propagated and that is the key point where individual-based models come to help to shed light over the inner intricacies of the process. The most common strategy for modeling conjugation was some variation of differential equations with mass action kinetics using whole population data for model calibration. But one of the main drawbacks of this approach is that it is a failure taking into account local variations and assume well-mixed environments which clearly are not realistic assumptions. In order to produce a more structurally realistic representation for conjugation, a spatially explicit individual-based model is required where agents have their own individual internal state and interact only with their closest neighbors being the changes in spatial position of agents exclusively consequence of the shoving relaxation due to the colony growth process.

Plasmids are mobile genetic elements, ubiquitous to the vast majority of prokaryotes, which can be seen, in some extent, as bacterial symbionts in all those cases when they confer some competitive advantage to their host or simply as plain parasitic DNA entities, using the cellular machinery only to their own persistence without providing any fitness improvement. Thereby, in the absence of any selective sweep favoring infected cells, plasmids are kept stably in bacterial populations but, normally, without a 
complete dominance. As general rule, on liquid medium, the frequency of conjugative events is low, chiefly owing to the random distribution of donor and recipient cells and to the difficulties to make stable mate aggregates. On the other hand, in structured colonies such as agar plates or biofilms, these aforementioned constraints do not exist, but nonetheless plasmids are also not able to completely infect the whole colony as could be intuitively expected.

The causes of such behavior are not clear but it seems proper to think that there should is some kind of feedback balancing loop, besides of the intrinsic delays, limiting the total amount of conjugative events. Some of the aspects having a net effect decreasing the frequency of conjugative events are the surface and the entry exclusion systems which avoids the same plasmid being transferred many times to an already infected cell [TN05]. Another factor limiting plasmid spread is the segregative loss, but usually it is considered to be a very low value affecting primarily small plasmids with high copy number, thus the probability of some plasmid being lost is inversely proportional to their copy number. Larger plasmids have a low copy number and present more sophisticated schemes to ensure their stability, such as the active partitioning, the postsegregational cell killing and the multimer resolution systems, being very unlike that a cell does not receive their plasmid copy [ $\left.\mathrm{SBH}^{+} 05\right]$.

Plasmid infection rates are also affected by the alternation periods of transitory derepression and repression cycles. Transitory repression is deemed to last a few generations facilitating the plasmid maintenance. But even plasmids having the pilus synthesis constitutively derepressed, which represents the optimal conditions to undertake successful conjugative events in spatially structured colonies, are unable to completely infect if nutrients are not replenished [FZKT08]. This seems to indicate a common mechanism, whose function is preserved in the different systems, which represents a barrier to plasmid invasion. This barrier appears to be indirectly related to the availability of nutrients that in turn controls the growth process.

\subsection{Summary}

In this chapter, we have provided a very brief overview about the main functional aspects of bacterial cells in order to make minimally intelligible the modeling approaches used subsequently in this work for creating the agent-based computational represen- 
tation of a bacterial cell. The topics enclosed in this chapter, such as the physiology and the biophysical aspects of bacterial life are by far too vast for being fully discussed, nonetheless it should be enough to getting a broad picture for remaining of this manuscript. 


\section{3 \\ Chapter

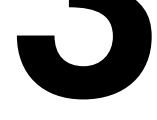

\section{Models and Conjugation}

3.1 Overview . . . . . . . . . . . . . . . . . . . . . . . . . . . . . . 42

3.2 Whole population models of conjugation . . . . . . . . . . . . . . 42

3.3 Individual-based models of bacterial growth . . . . . . . . . . . . . 45

3.4 Individual-based models of conjugation . . . . . . . . . . . . . . 49 


\subsection{Overview}

The objective of this chapter is to provide a brief overview about the existing efforts for modeling the kinetics of plasmid spread in order to contextualize the contribution of this work. Despite of the great importance of bacterial conjugation as the main vector of the generalized spread of multidrug resistance, the number of studies addressing the systemic aspects of that form of lateral genetic transfer are rather scarce. Certainly, it is a consequence of the relative youth of the field which dates back to the middle of the forties with the studies about genetic recombination in E. coli [LT46] being the term plasmid coined later [Led52] [Sum96].

\subsection{Whole population models of conjugation}

Chronologically, the first attempt to explain the kinetics of conjugative plasmid using mathematical model based on ordinary differentia equations was a study about why this types of mobile genetic elements are able persist over the time in bacterial populations [SL77]. Subsequently, the same authors of the previous study have proposed the classical work on the field where the dynamics of bacterial conjugation is assumed to follow a mass action model [LEV79] which is shown in the Equation (3.1).

$$
\begin{aligned}
\frac{d n_{r}}{d t} & =\psi n_{r}-\gamma\left(n_{d}+n_{t}\right) n_{r} \\
\frac{d n_{d}}{d t} & =\psi n_{d} \\
\frac{d n_{t}}{d t} & =\psi n_{t}+\gamma\left(n_{d}+n_{t}\right) n_{r}
\end{aligned}
$$

where $n_{r}, n_{d}$ and $n_{t^{1}}$ are respectively the population density in cells $/ \mathrm{ml}$ of plasmid free, donors and transconjugant cells. The parameters $\psi$ represents the rate of growth and $\gamma$ the conjugation rate. The model has been used later for study the range of

\footnotetext{
${ }^{1}$ The notation employed originally in the paper was $n, n_{+}$and $n_{*}$ for plasmid free, donors and transconjugants but we have substituted in this work for making it consistent with the current terminology used here for population categories $\mathrm{R}, \mathrm{D}$ and $\mathrm{T}$.
} 
conditions which allows the permanence of plasmids in bacterial populations. The underlying assumption was that plasmids are detrimental for the fitness of their hosts and that the plasmids are susceptible for being eliminated by vegetative segregation across successive divisions [BLL00].

It can be easily observed, taking into account the structure of the system of ODE shown Equation (3.1), that the model is using the same growth rate for all types of individuals and the underlying assumption is that the rates of change in the population depends on the totality of individuals, as can be seen in the term $\gamma\left(n_{d}+n_{t}\right) n_{r}$ which basically states that rate of transconjugant cells are increasing is a function of totality of donors, transconjugants and recipient bacterial cells represented by the state variables $n_{d}, n_{t}$ and $n_{r}$. This obviously is ignoring any type population structure and patchiness assuming a completely homogenous colony in a constant mixing, an assumption which clearly does not hold. Additionally, this kind of modelling approach offers no mechanistic or qualitative explanation about what is going on in the low level bacterial colony structure.

This basic seminal model was further extended in several ways, one of them by considering the nutrient consumption required for producing a new bacterial cell [SGSL90], the differentiate growth rates for each of the sub-population types, as well as the cost imposed by the plasmid bearing as the net reduction of infected cell growth rate [BLL00]. The ODE term added to system for capturing the substrate dependency [SGSL90] is $d C / d t=-\psi(C)(R+D+T) e$ where $e$ represents the amount of substrate which is needed by generating a new bacterial cell in micrograms $(\mu g)$. The growth rates and the conjugation rate are made dependent of substrate uptake using a Monod function as can be seen in Equation (3.2) for both terms.

$$
\begin{aligned}
\psi(C) & =\psi_{\max } \frac{C}{K+C} \\
\gamma(C) & =\gamma_{\max } \frac{C}{K+C}
\end{aligned}
$$

where $\psi_{\max }$ and $\gamma_{\max }$ are the maximum values of growth rate and conjugation rate

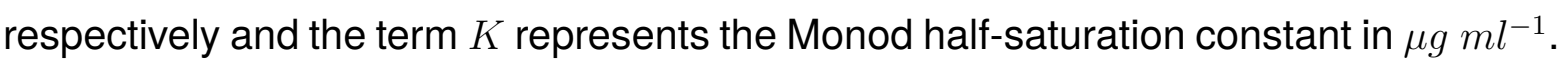
Including in the model differentiate growth rate for the distinct population states 
allowed the evolutionary ecology study of the conditions required for the plasmid persistence at evolutionary time keeping only a parasitic relationship with bacterial hosts, concluding that in the absence of beneficial traits, plasmids should be eliminated from bacterial populations. Interestingly, that kind of conclusions drawn from the model results could be seen as consistent with the competitive exclusion principle [Har60] [BTH06] but are consequence of failing to take into account the spatial structure of the bacterial colony which gradually imposes spatial separations between the plasmid bearing and plasmid free cells leading to the formation of demes. Actually, a much more plausible explanation is that in structured populations, the plasmid bearing cells and plasmid free cells become physically separated and consequently not compete with each other which could in some extent explain the parasitic persistence.

One of the main drawback of whole-populations models is that they offer very few information about the underlying process. Taking this into account, another extension of the previous basic model has been proposed for including more structural information into the model, separating the functional elements of conjugative process[ZKaTK10], such as the attachment of to two cells in the mating pair formation, the DNA transfer and finally, the detachment of cells. The environmental aspects are also contemplated by including in the model the effects of mixing intensity. All of these processes have been considered in the model by the addition of new terms to the ODE system as can be seen in the Equation (3.3).

$$
\begin{aligned}
d R / d t & =\psi_{R}(C)\left(R+M_{1}+M_{2}\right)+k^{-}\left(M_{1}+M_{2}\right)-k^{+} R(T+D) \\
d T / d t & =\psi_{T}(C)\left(T+M_{1}+M_{4}+2 M_{3}\right)+k^{-}\left(M_{1}+M_{4}+2 M_{3}\right)-k^{+} R T \\
d D / d t & =\psi_{D}(C)\left(D+M_{2}+M_{4}\right)+k^{-}\left(M_{2}+M_{4}\right)-k^{+} R D \\
d C / d t & =e\left[\psi_{R}(C) X_{1}+\psi_{T}(C) X_{2}+\psi_{D}(C) X_{3}\right] \\
d M_{1} / d t & =k^{+} R T-k^{-} M_{1}-\gamma_{T}^{0}(C) M_{1} \\
d M_{2} / d t & =k^{+} R D-k^{-} M_{2}-\gamma_{D}^{0}(C) M_{2} \\
d M_{3} / d t & =\gamma_{T}^{0}(C) M_{1}-k^{-} M_{3} \\
d M_{4} / d t & =\gamma_{D}^{0}(C) M_{2}-k^{-} M_{4}
\end{aligned}
$$


where the terms $M_{1}, M_{2}, M_{3}$ and $M_{4}$ represents respectively the densities of attached pairs of $(T, R),(D, R),(T, T)$ and $(D, T)$, it is worth to note that in the original formulation these terms have a different representation, $M_{T R}, M_{D R}, M_{T T}$ and $M_{D T}$ - Also, the terms $X_{1}, X_{2}$ and $X_{3}$ are simply a shortened form of $\left(R+M_{1}+M_{2}\right)$, $\left(T+M_{1}+M_{4}+2 M_{3}\right)$ and $\left(D+M_{2}+M_{4}\right)$ respectively ${ }^{2}$. The other components of differential equation system have the same meaning already discussed previously.

The parameters $k^{-}$and $k^{+}$are the detachment and the attachment rates respectively which are both affected by the intensity of mixing, these parameters could be seen as similar to the bulk conjugation rates[ZKaTK10] calculated using the end-point method[SGSL90]. The detachment rate is directly proportional to the mixing and the attachment rate is favored by lower mixing speeds, becoming stable as the mixing intensity increases. It is interesting to note, that this work has used for fitting the model parameters, only one experimental variable, namely the shaking speed of culture which affects the attachment and the detachment rates. The remaining model parameters were kept fixed with the same values for the growth rates and the intrinsic conjugation rates of recipient, donors and transconjugant cells, respectively $\psi=1.7$ and $\gamma^{0}=15$ for the simulated plasmids F, pB10 and R1. The value of 15 for the intrinsic conjugations rates is attributed to the average time of 4 minutes required for conjugative transfer. The work does not indicate that any kind of systematic parameter estimation methodology have been applied.

\subsection{Individual-based models of bacterial growth}

In the previous section, we have discussed the continuous approaches for modeling the bacterial conjugation and here we will provide a general overview about the currently available efforts for the agent-based discretized representation of lateral gene transfer process. Owing to the fact that the bacterial conjugation is not occurring isolated from other cellular activities, it is necessary to take into account at least a minimal set of cellular sub-process for making the conjugation dynamics consistent with the colony growth kinetics. For that reason, we will also review some foundational and representative agent-based works for simulation bacterial colonies.

\footnotetext{
${ }^{2}$ We had rewritten some terms of the original equation for making it short
} 
Chronologically, the first spatially individual-based model of bacterial colony growth incorporating a detailed and fine-grained quantitative representation of intracellular processes was BacSim [KBW98]. Previously, a lattice-based model has been proposed for exploring the cooperative and chemotactic aspects of bacterial colony development[BJST ${ }^{+}$94] but the modeling unit was what the authors termed walkers, technically a mesoscopic entity representing an aggregate, not an individual bacterium although conceptually these entities resemble some points of bacterial metabolism and nutrient uptake. The spatial patterns generated model have been comparted to experimentally tuned cultures using different ranges of nutrient availability and substrate consistency which limits the chemotactic random walks showing a good agreement.

Returning to the BacSim model, it is worth to noting that the model has fundamentally all components for describing the most significant aspects of bacterial life cycle which have been conserved in most of its descent models. The model uses 2D continuous representation and considers bacterial cells as spherical entities which incorporates the following cellular sub-process: the substrate uptake, the cellular housekeeping represented by the metabolism and maintenance, the cellular division and the starvation and eventual cell death when the current cellular volume is less than the minimum nominal value[KBW98]. Additionally, the model also includes processes representing the diffusion of nutrient substrate and the colony expansion by relaxing the forces generated due to the cellular overlapping.

The model requires the input of eight different parameters for the set of equations which updates the state of agents. These parameters are (1) the maximum growth rate $\mu_{\max }\left(\mathrm{min}^{-1}\right)$; (2) the Monod half-saturation constant $\left(K_{s}\right)\left(f g f l^{-1}\right)$; (3) the apparent yield $\left(Y_{\max }\right)$ at $\mu_{\max }$ (dry mass/glucose) being both terms in femtograms; (4) the apparent maintenance rate $m$ at $\mu=0$ expressed in units of glucose/(dry mass $\times$ min), again glucose and dry mass in femtograms; (5) the median cell volume $\left(\bar{V}_{u}\right)$ at $\mu=0$ expressed in femtoliters; (6) the time for replication + cell division in minutes; (g) the minimal cell size in femtoliters; (7) the cell density in femtograms/femtoliters; and finally (8) the glucose substrate diffusion coefficient in $\mu \mathrm{m}^{2} \mathrm{~min}^{-1}$. The model updates the internal state variables of its agents by solving a set simple differential equations using the previous parameters.

The previous model, in its ensuing version (BacSim version 2), have been extended for adding the required support simulating the development of bacterial 
biofilms[KPWvL01]. Generally the basic model structure are very similar in both versions but including more parameter and state variables. Differently from its previous version the model represents the biofilm formation in an emulated 3D geometry for presentation purposes, even though the simulation system is still 2D. The computational domain is represented by a base layer with a substratum where the cells attach and a bulk liquid separated by a boundary layer which moves up as the biofilm grows.

The previous model, in its the subsequent version (BacSim version 2), have been extended for adding the required support simulating the development of bacterial biofilms[KPWvL01]. In general, terms the basic model structure are very similar in both versions but including more parameter and state variables. Differently from its previous version the model represents the biofilm formation in an emulated 3D geometry for presentation purposes, even though the simulation system is still 2D. The computational domain is represented by a base layer with a substratum where the cells attach and a bulk liquid separated by a boundary layer which moves up as the biofilm grows.

In the same line with the previous simulator and also including some aspects in common with it, was introduced the Individual Discrete Simulations or simply INDISIM[GLV02]. This model encompasses similar processes for implementing the rules used in the update of the state variables. The processes are the nutrient uptake, the metabolism, the cellular viability and the cellular reproduction. But differently from BacSIM, INDISIM uses a discrete space representation, being the computational domain, a discrete lattice of $100 \times 100$ and update the state variables drawing random numbers for the model parameters instead of solving differential equations. Differently, from BacSim which relies on Monod, INDISIM uses a simpler Blackman kinetics for capturing the substrate-limited growth rate $\mu(C)$. The model has the following parameters: (1) the maximum consumption $\left(U_{\max }\right)$; (2) the standard deviation of $U_{\max } ;(3)$ the percentage of available nutrient particles $(k)$; (4) the metabolic efficiency $(\mathrm{Y})$; (5) the enthalpy coefficient $(\mathrm{K}) ;(6)$ the residual product $(\mathrm{E}) ;(7)$ the minimum and maximum value of $\mathrm{CO}_{2}$ particles; (8) the average values of reproduction mass $\left(M_{R}\right)$; (9) the standard deviation of $M_{R}$ and finally (10) the cellular cycle time $t_{R}$.

Another existing tool is the AgentCell simulation software which has been developed using the Repast framework for $\left[\mathrm{NCO}^{+} 13 \mathrm{~b}\right]$ creating a model of the chemotaxis process using a $3 \mathrm{D}$ computational geometry[ $\left.\mathrm{EMN}^{+} 05\right]$ and trying to unravel how the 
intracellular random noise affects the whole cell response. It is also worth to mention that the model is focused on simulation the chemotactic behavior of $E$. coli cells using massively parallel computers and do not have and user interface which certainly limits its general use.

The BacSim model, have been superseded by an improved version denominated individual-based Dynamics of Microbial Communities Simulator or simply iDynoMICS[LMM $\left.{ }^{+} 11\right]$ which, according to the purpose statement, from the its ODD protocol[ $\left.\mathrm{GBD}^{+10}\right]$, the main objective of this software is to simulate the growth of microbe populations, taking into account the competition for nutrient resources and space, in biofilms. The standard version includes two agent types, namely the bacterial agents and the extracellular polymeric substances (EPS) but it is possible to add more agent types if needed. The sub-process structure for the bacterial life cycle is quite similar to the previously described for its predecessor. One of the salient features of iDynoMICS is the possibility to add customized metabolic reactions to the agents, because differently from previous version it is no longer hardcoded in the model structure. In the current default version, bacterial agents spherical-shaped entities but it is also possible to use cylinders for representing the simulated cell. The standard version has been extended, in an alternative code branch, for supporting rod-shaped bacterial cells[Álv12] as well. Another interesting feature, added outside of the group which have developed originally the simulator, is the possibility of modeling the bacterial conjugation process[Gar13].

Another extensible agent-based modeling for the simulation of bacterial populations is the BSim software which is targeted as a synthetic biology tool[GMT $\left.{ }^{+} 12\right]$. The software includes a set of reusable built-in bacterial traits which can be seen as "chassis" for supporting more complex functionalities. The simulation tool also includes a facility for adding to the agents simulated genetic regulatory networks based on ordinary differential equations. It has a modular design allowing to add new types of agents by extending the basic agent type, thus it is no limited to simulate only bacterial cells but can be used even for modeling individual molecules.

Finally, recently a very well structured and described individual-based model has been proposed for simulating the effects of intercellular competition for the substrate access[TLNV15]. That kind of model, despite of its specificity are very useful for providing a very structured approach for describing each of the bacterial sub-processes 
which is tremendously useful for the cross validation of different model implementations. The mode also includes a realistic, accurate an efficient implementation for solving numerically the law of Fick for two-dimensional diffusion process.

\subsection{Individual-based models of conjugation}

The horizontal genetic transfer process, as we have already mentioned previously, is the root cause of the rapid spread of antibiotic resistance phenotypes across bacterial strains and an important piece in for the action of evolutionary forces shaping the genome of prokaryotes[ $[\mathrm{HH} 16]$, especially in the case of plasmids with a high copy number. Additionally, it is an essential tool in synthetic biology for introducing chimeric or foreign DNA into different cell types for expressing gene products[Kea08][KC10]. But nevertheless, the list of computational representations using individual-based approaches are very short and additionally none of these models are homogenous with respect to the structural aspects of conjugative process.

Perhaps one of the first efforts for overcoming the limitations derived from the unrealistic assumptions of models based on ordinary differential equations was achieved by modeling the bacterial conjugation using an asynchronous cellular automaton[ $\left.\mathrm{KLF}^{+} 07\right]$ which allowed to capture the spatial structure developed during the colony growth. The model belongs to a family of models denominated interacting particle systems [MCO05] or IPS which uses a continuous time and a discrete space representation. This model could capture one of the main constraints imposed to the plasmid transfer process, that is to say, the infection can only take place between close neighbors and the conjugative encounters are no longer random involving all population members but instead, restricted to a small number of suitable recipients on the neighborhood of donor cell. It was a tremendous qualitative leap for providing a more structurally consistent computation representation of conjugation. Additionally, the model also incorporates the competition for nutrients and space which usually arises during the colony development. The model makes the conjugation dependent on the nutrient availability which seems to be consistent with real behavior. It is assumed a $3 \times 3$ neighborhood (Moore neighborhood) for conjugative transfers from donors and transconjugant cells to plasmid free recipients. This model represents the conjugative process using the rates $\gamma_{D}(C)$ and $\gamma_{T}(C)$, respectively the conjugation rates of donors and transconju- 
gants, both dependent on the nutrient availability. The plasmid free cells at a lattice site $x$ are then infected with the rate shown in Equation (3.4).

$$
\gamma_{D}(x, C) n_{D}(x)+\gamma_{T}(x, C) n_{T}(x)
$$

the terms $n_{D}(x)$ and $n_{T}(x)$ are the number of donors and transconjugant cells on the Moore neighborhood of site $x$ and the parameter $C$ represents the amount of nutrient available on the nutrient neighborhood, again a Moore neighborhood with size $7 \times$ 7 lattice sites. The $\gamma$ rates must not be misinterpreted as bulk rates, instead these rates are basically the inverse time for the occurrence of a conjugative event. The functional dependency $\gamma(x, C)$ between the value of conjugation rate and the nutrient concentration is defined using the known blackman kinetics[DFW73].

Subsequently, another study carried out by the same research group, using a mix of experimental and mathematical modeling approach[FZKT08] has been able to identify the most significant factors which are responsible for the extent of plasmid dissemination when the initial frequency of plasmid bearing cells was very low, in the order of $10^{-7}$ and additionally, no positive traits are conferred to its hosts. These factors, seems to be the spatial structure and the nutrient availability which are shaping the epidemic pattern of plasmid spread. The model is also a stochastic cellular automaton as in the previous one[ $\left[\mathrm{KLF}^{+} 07\right]$ but including some 3D features, allowing the presence of several levels of bacterial cells on the same lattice site. The conjugation, in the same way as in the previous model, does not depend on the internal state of bacterial cell but instead it is a function of the number of infected cells on the sites surrounding the focal point.

Thereafter, another study carried out by the same research group, using a mix of experimental and mathematical modeling approach[FZKT08] has been able to identify the most significant factors which are responsible for the extent of plasmid dissemination when the initial frequency of plasmid bearing cells was very low, in the order of $10^{-7}$ and additionally, no positive traits are conferred to its hosts. These factors, seems to be the spatial structure and the nutrient availability which are shaping the epidemic pattern of plasmid spread. The model is also a stochastic cellular automaton as in 
the previous one[ $\left[\mathrm{KLF}^{+} 07\right]$ but including some 3D features, allowing the presence of several levels of bacterial cells on the same lattice site. The conjugation, in the same way as in the previous model, does not depend on the internal state of bacterial cell but instead it is a function of the number of infected cells on the sites surrounding the focal point.

The prior studies use a somewhat artificial approach for representing the conjugative process which is modeled without considering any structural aspect about the bacterial cell for implementing the decision rule for infecting a recipient cell. Despite of being formally consistent these types of models are unable to provide satisfactory explanations about what are the cellular mechanisms responsible for the observed kinetics and additionally most of the model parameters have absolutely no biological meaning which seriously limits the extrapolation of model results for other plasmid-host combination without repeating the experimental setup and fitting the model parameters again. That situation has changed with the publication of a novel work describing the results gathered from the in vivo direct observation of the horizontal genetic transfer process for the combination Pseudomonas putida host and the pWWO TOL plasmid[SYD+11]. The results pointed out that the conjugative events are rare on the early phases of envelope elongation in such extent that the most of conjugative events have been detected when the bacterial cell have achieved at least a $70 \%$ of its total growth before division. Another interesting result shows that the conjugal activity requires a tight contact between plasmid donors and recipients, so that approximately a $90 \%$ of successful transfer are likely to happen in a range from 0 to $1 \mu \mathrm{m}\left[\mathrm{SYD}^{+11}\right]$. Finally, it has also been observed that the conjugation activity is significantly impaired if the bacterial cells have the division disrupted even when nutrients are not completely depleted. These results have provided very important clues for subsequent models and paved the way for a more consistent computational implementation for the plasmid transfer.

The experimental study mentioned above inspired the development of an individualbased model for exploring the dependency between growth rate and conjugative transfer[MLS $\left.{ }^{+11}\right]$. The working hypothesis is settled over the assumption that the plasmid transfer is linked and depends on the ATP production rate which in turn has a functional dependency from the whole cell growth rate. The model represents this dependency making the conjugative activity associated to the pilus scan speed as fol- 
low: $v_{s}=v_{s}^{\max } f(\alpha)$ where $v_{s}$ is the pilus scan speed and $\alpha=\mu_{\text {obs }} / \mu_{\max }$. The model also incorporates explicitly, as initialization parameters, the maturation time and the recovery time. The conjugative process uses a probability parameter $p$ for deciding whether conjugate or not when a suitable recipient is found and in the simulations carried out the authors used a value of $p=1$ meaning that all encounters are successful. The simulation output has been systematically analyzed using the sensitivity analysis methodology and the results have shown that the most important parameters are those related to the timing factors affecting, what we call in this work, the propagation or forwarding delay. Specifically, the most significant parameter was the plasmid maturation time which effectively limits the speed of propagation wave across the bacterial colony.

One of the aspects which severely limits the comprehensive interpretation of the different results obtained from the existing models as well as the comparison of individualbased model outputs which those produced by mean field models, is precisely the lack of a common metric for expressing the efficiency of conjugative process. The reason is that the bulk conjugation rates taken from ordinary differential equations, that is to say, the so called $\gamma_{e p}$ are not directly comparable to the individual based conjugation rate $\gamma$. Bearing this in mind, it has been proposed[ZZFF+12] a conversion scheme from cells/lattice to cells/ml and vice versa, defined as $\bar{N} / L^{2}$, where $N$ is the assumed carrying capacity of one milliliter of solute and $L^{2}$ the number of lattice sites.

Recently, another individual-based approach named DISCUS[GMA15] for modeling the bacterial conjugation has been proposed bearing in mind the simulation of distributed genetic programs and its distribution as embedded as conjugative plasmids. The software has put the focus on providing and accurate physics for capturing the underlying the colony expansion, representing it as a rigid body system. This model implements the conjugation rule as a discrete probability distribution, described by the authors as $C\left(N, p, c_{t} i m e\right)$, being $N$ the number of trials during the cell cycle, $p$ the success probability on a trial basis and $c_{t} i m e$ the interval where $p=0$. This model also adheres to the idea that exist a positive correlation between the minimum elongation and the frequency of conjugative events[SYD+11], using a parameter which the authors termed cell $_{i} n$ fancy which actually limits the conjugative transfer before that period. This is consistent with the results provided by a previous individual-based model which explores the linking between and cell cycle and conjugative events[Pre11]. 


\section{Part III}

\section{The Research Problem and the Methodology}





\section{Chapter

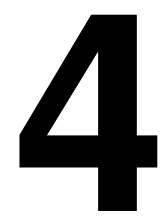 \\ The challenging of modeling the bacterial conjugation}

4.1 Overview . . . . . . . . . . . . . . . . . . . . 56

4.2 The research problem $\ldots \ldots \ldots \ldots$

4.3 Methodology $\ldots \ldots \ldots \ldots$ 


\subsection{Overview}

The task of building individual-based models for gathering qualitative results which could shed light for interpreting the internal local structure responsible for the globally observed behavior, have a high level of complexity. This is mainly due to the fact that the vast majority of parameters required to model the intra-cellular behavior of bacterial cells cannot be observed directly. This is known as parameter uncertainty standing for those parameters used in computer models for which the actual exact values are unknown and cannot be controlled [GR05a].

The most adequate way for tackling with cellular complexity is adopting a divide and conquer approach, which in that context means to provide a modular abstraction for the bacterial cell process, constructing sub-models for every process which are carried out by living cells and simplifying the computational representation whenever it does not implies losing important details. As a general rule, adding many details to achieve a complete implementation would represent an extremely high computational cost making completely impractical to simulate a representative number of individuals required to produce accurate results. Therefore, the right choices are required to be made, selecting only those processes which are significant for the aspect under study.

In addition of identifying and implementing the right set processes special attention must be paid on their temporal scales as well as on how these processes are linked together in order to capture the systemic structure. It is important to note that living cells are a bunch of biochemical reactions occurring in parallel at distinct timescales and failing in capture that feature will produce inconsistent global dynamics.

Thereby our model can be represented by the sum of several physiological and biophysical sub-processes that can be readily identified at first glance as being the most representative for cell cycle. These processes are fundamentally the nutrient uptake, the metabolization of nutrient particles and its incorporation to the cell mass, the chromosome replication, the expression of a set of genes which are not part of basal metabolic activity, including those related to the expression of conjugative apparatus, the plasmid replication and the maintenance of its copy number.

Temporal accuracy on every simulated sub-process is determinant for getting valid results in the simulated conjugation rates owing to the fact that the most important component in the plasmid spread speed are the parameters related to time and delays 
in the infection network.

All of these sub-processes included in our model can be implemented in many different ways depending on the feature we are trying to capture in the simulation model. If the only objective is to get the intercellular competition for nutrient, it may be enough to use a simple implementation making the computational representation of bacterial cells to uptake one nutrient particle every time a daughter cell is generated. That approach can be found in [KLF+ 07$]$ where the amount of nutrient is one per lattice cell. That implementation can be improved making more nutrient particles available. The empirical rule for estimate the total quantity of nutrient particles is based on the total time we want to simulate. Hence, if we assume saturating conditions we will use approximately a number of particles equal to the total simulated time divided by the expected generation time.

\subsection{The research problem}

Generally speaking, the problem being addressed in a first instance in this work is the development of a usable computational model for the bacterial conjugation process which could be safely applied for a large set of conditions. Thus, in order to ensure the model is operational, it is necessary to achieve a good consistency level between simulated and the reference data, obtained from systematic laboratory experimental setups. Additionally, the model should be capable to facilitate the development of qualitative explanations for the structure of the intra and inter cellular process governing the global dynamics allowing the generalization and the subsequent application for the simulation more complex situations for which there are no direct experimental observations, such as the unculturable strains or complex microbial communities and different plasmids and host associations. Consequently, the modeling approach will hopefully generate a system level knowledge about the conjugative plasmid spread relaying on the structural characteristics which are already available for many hosts and plasmids and inferring those which are common or can be assumed to be conserved in different conjugative systems and bacterial strains.

More specifically, the ultimate goal is being able to produce trustworthy predictions using the structural properties bacterial hosts and the associated plasmids as the model parameters for describing quantitatively the global dynamics which is most li- 
kely of being generated taking into account these properties and the initial conditions eliminating the requirement of building a wet lab experimental design for having the quantitative data for every host-plasmid combination. Certainly, this is very ambitious goal and cannot be attacked efficiently in one step because of the elevated cellular complexity and the associated uncertainty. Therefore, in this work, we focus on elucidating two main aspect which seems to determinate the global observed behavior and the epidemics character of conjugative process, namely the specific point in the cell cycle when the plasmid transfer is most likely to happen as well as the associated metabolic costs of the whole process and its subsystems. A third problem will be partially outlined and is related to the linking between the plasmid structural properties and how they are related to the previous first two key points. Briefly, the specific research questions which are addressed directly, laterally or simply outlined in this thesis are presented on the box shown below.

\section{The key research problems addressed directly or laterally in this work}

Q1: When the conjugative transfer is most likely to happen during the bacterial cell cycle?

Q2: Is it significant for the global plasmid spread dynamics?

Q3: What biophysical factors are contributing for the conjugative dynamics?

Q4: How the combination host-plasmid affects the plasmid dispersion?

Q5: What are the most plausible range of values for the plasmid metabolic burden, including the costs related to the conjugative process and the expression of genes which are essential for the plasmid transfer?

Q6: Why there are several different metabolic costs reported for the same combination of conjugative system and hosts?

Q7: What parts of cost component system are reduced by the coadaptation of host-plasmid system?

Q8: How conjugative plasmids use a mixed strategy combining vertical and horizontal transmission?

Q9: What are the effects of spatial separation and the genetic drift in the overall conjugative efficiency? 


\subsection{Methodology}

The methodology employed for achieving the specific goals mentioned previously is settled over the development of computational models and the analysis of model outputs, comparing them with the data obtained from cytometric experiments [ $\mathrm{dCRC}^{+} 12$ ]. Fundamentally, three main complementary techniques are used together for understanding the factors linking the local and intracellular aspects of bacterial cells with the global dynamics measured at a whole-population level. These techniques are the individual-based modeling [GR05a], the parameter estimation [TKG14] [BA77] [ZR06] and the sensitivity analysis of computation models [ $\left.\mathrm{PBF}^{+} 16\right]$ [STCR04] and they are used iteratively for continuously improving the quality of predictive aspects of model implementation. The initial model formulation uses the available knowledge on the bacterial cell structural properties for representing the single cell behavior as ensemble of empirically consistent set of sub-processes integrated in way that the resulting model is empirically consistent, honoring the parsimony principle which mandates to pick just the components which are essential for representing the target of modeling process $\left[\mathrm{PPH}^{+} 12\right]$.

The research procedure encompasses the development and the evaluation of multiple alternative implementations for the base model. The software required for simulation and analysis has been developed explicitly for this work. The bacterial colony simulation has been primarily written in Java using the Repast agent-based modeling framework [ $\left[\mathrm{NCO}^{+} 13 \mathrm{a}\right]$ and the most of software for analyzing the model output has been implemented using GNU R [R C15] [Cra07] and small parts in MATLAB. The development lifecycle begins with the implementation of a model incorporating the standard set of components required for simulating a bacterial colony growth which are the nutrient uptake, its metabolization, the vegetative growth and division as well as the associated cell pushing process allowing the colony expansion. The alternative set of rules capturing the different empirically plausible mechanisms for the horizontal gene transfer is added on the top these sub-processes and submitted to analysis sieve.

Initially, the implementation is assessed informally, observing whether the colony growth shows the observed spatial patterns, including those related to the sector formation with subpopulations containing only a bacterial type. Subsequently, the quantitative growth and the conjugation rate time series are compared and contrasted against 
the reference data [ $\left.\mathrm{dCRC}^{+} 12\right]$ and observations [SYD $\left.{ }^{+} 11\right]$ using and initial set of plausible parameters for the model. In case of model provides a good and smooth adjustment to the experimental data the next step is the systematic search of parameter space and the formal verification of model behavior based on a what-if approach carried out using the sensitivity analysis procedure. These later steps generate more insights about the model structure allowing a better understanding about the parameters which are not obtained directly and their associated uncertainty. Additionally, this could lead to the improvement of the initial model structure for instance eliminating parameters which are not representative in the total output variability. 


\section{Part IV}

\section{The Proposed Solutions to the Research Problem}





\section{Chapter}

\section{Parameter estimation}

5.1 Introduction . . . . . . . . . . . . . . . . . . . 64

5.2 Parameter Estimation and Optimization . . . . . . . . . . . 65

5.3 Metaheuristics for Parameter Estimation . . . . . . . . . . . 70

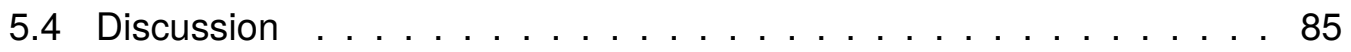

5.4 .1 Optimizing simple functions $\ldots \ldots \ldots \ldots$

5.4 .2 Tuning oscillations . . . . . . . . . . . . . . 87

5.4 .3 Exploring the solution space $\ldots \ldots \ldots \ldots$. . . . . 89

5.4 .4 Comparing metaheuristics. . . . . . . . . . . . . . 93

5.4 .5 Parameter estimation of individual-based models . . . . . . . 96

5.5 Summary . . . . . . . . . . . . . . . . . . . . 107 


\subsection{Introduction}

Modeling and simulation is certainly a vast discipline with a broad and complex body of knowledge having, beyond the surface, a large technical and theoretical background [Min65] [BCNN09] [ZPK00] [Boc03] which consequently, is hard to completely mastered by modelers coming from disperse domains like biology, ecology or even computer science. Among the existing formalisms, the agent-based or individual-based is increasing gradually the number of adepts in the recent years. The Individual-based modeling is a powerful methodology which is having more and more acceptance between researchers and practitioners of distinct branches from social to biological sciences, including specifically the modeling of ecological processes and microbial consortia studies. Certainly, one of the main reasons for the success of this approach is the relative simplicity for capturing micro-level properties, stochasticity and spatially complex phenomena without the requirement of a high level of mathematical background [GR05b]. But the counterpart of the ease for building complex and feature rich models, is the lack of a closed formal mathematical form of the model which implies that the study of these models cannot be attacked analytically. Thereby, the only way to explore and adjust the parameters of these type of models is the brute-force approach, executing the model many and many times and evaluating the results of each execution.

The systematic search for the best set of model parameters is a costly task for which there are basically two different types of approaches for exploring the solution space of simulation outputs. The first one is using some static sampling scheme based on the design of experiments (DoE), such as randomized, factorial or Latin hypercube [LH78] [Loh96] [TKG14] designs which works by generating a collection of sampling points of parameter space which are further evaluated. Alternatively, the parameter estimation can be also stated as an instance of an optimization problem and therefore, addressed more conveniently using the whole arsenal of metaheuristics and evolutionary strategies. The main difference between these two ways of tackling with the problem lies on the fact that the first one is fundamentally a static sampling technique whereas the second is an intrinsically dynamic form of a guided partial search over the solution space where the set of initial solutions are continuously improved and hopefully converging towards a global optimum [Wei09] [BLS13]. It turns out that comparatively, the optimization approach may require less model evaluations to find the best, or at least 
an acceptable solution for the parameter estimation problem which, in the case of models with an average complexity level, means a difference between an upper bound computational cost from hours to days.

The parameter estimation of individual-based models is a particularly hard instance of an optimization problem as they are highly stochastic and the parameter space most often tend to show nonlinear interactions which difficult the localization of good parameter combinations producing the minimal deviation from reference data. Differently from deterministic and closed form models, where the most significant computational cost is due to the optimization algorithm itself, in the case of individual-based models the time required for every single model execution alone is responsible for most of the computational time taken in the optimization process. It is important to consider that some metaheuristics may produce better results than others depending on the problem type and more specially on the structure and characteristics of the model being analyzed. Therefore, it is interesting before undertake a full length run to explore different algorithms in order to find the best suited for the problem instance.

In the next sections, we will briefly describe the scope of parameter estimation problem and the usage examples of the EvoPER R package which has been developed for facilitating the tasks of estimating the parameters of Individuals-based models. The current version of EvoPER includes implementations of Particle Swarm Optimization (PSO), Simulated annealing (SA) and Ant Colony Optimization (ACO) algorithms developed exclusively for this work and adapted for their use in the parameter estimation of agent-based models. We are also introducing two simple evolutionary strategies implemented for exploratory analyses for the parameter space of individual-based models which can be useful for mapping the promising zones of solution space.

\subsection{Parameter Estimation and Optimization}

The terms model calibration and parameter estimation, although informally are used interchangeably and being functionally similar are semantically distinct entities having a different scope and objectives. Therefore, to provide a more formal definition of these terms let us briefly define the basic structure of a mathematical model. A model is normally expressed as some form of the algebraic composition expressing the relationship between of three element types, namely the independent variables, the 
dependent or the state variables and finally the constants. For the sake of simplicity, a model expressing some linear relationship between variables is shown bellow

$$
y=\alpha+\beta x
$$

where $x$ and $y$ are the independent and the state variable respectively and $\alpha$ and $\beta$ are the model constants. More generally, the structure of and stochastic model can be represented by the functional relation [Hae96] given by the expression shown in the Equation (5.1),

$$
y=f(\vec{x}, \vec{p}, \vec{\epsilon}),
$$

The terms $\vec{x}, \vec{p}$ and $\vec{\epsilon}$ denotes respectively the vectors of independent variables, the vector of model parameters and the stochastic deviations.

The model constants are referred as the model parameters which necessarily do not have to have any correspondence to some element in the system being modeled [BA77]. The direct problem is, being known the model structure and, also knowing the independent variables and the parameters, to estimate the value of state variable. Of course, this oversimplified case is rarely seen when modeling real systems, especially when dealing with biological systems. In addition, in the most cases the constants and the independent variables are impossible to observe directly being also unknown the right model structure for representing the system under study.

Usually the only value elucidated experimentally or backed by observations of some population data is the state variable; therefore, the parameters which are the structural part of model must be estimated having as the only reference, the measurements of dependent variable. Hence the term calibration can be defined as the procedure to where the values of state variable " $y$ " are compared to the known standard values, let's say " $Y$ ", which in the context of biological research are those sampled from population true values [ZPKO0].

On the other hand, the parameter estimation is the task of estimating the values of the constants of a model and it can be seen somehow as an inverse problem, since we are using the reference values $Y$ in order to determine the suitable values for the model constants [AFNKB09] [BA77]. The parameter estimation procedure implicitly encom- 
passes the calibration process as, in order to discover the values for the constants the model outputs must be checked to the reference values. Thus the problem can also be stated as an optimization problem, just because the process requires the search for the minimum values of some function $f\left(y_{i}, Y_{i}\right)$ measuring the distance between $y_{i}$ and $Y_{i}$ which are the simulated and the reference values respectively.

The family of functions measuring how close are the simulated and the reference values is the goodness of fit metric of a model and is known as the objective function. The objective function facilitates the determination of how well the model is able to reproduce the reference data. In other words, the objective function provides a numerical hint about how close are the output of the model to the reference data. For any given model, a family of different objective functions can be defined over the output data, depending on the chosen distance metric and on what is the target of parameter estimation process. More formally, the objective function can be defined as $f: \mathbb{R}^{n} \rightarrow \mathbb{R}$ and can be further generalized for an individual-based model where, differently from a pure mathematical form, the objective function domain may assume any valid computational type. Thus, being $\mathbb{S}$ the set of valid computational model parameters, the objective function can be rewritten as $f: \mathbb{S} \rightarrow \mathbb{R}$.

Therefore, every candidate solution $x$ is instantiated from the solution set $\mathbb{S}$ being the best of them known as the solution or the optimal and represented as $x^{*}$. Hence, the target of optimization process is to find the solution $x^{*}$ which minimize the objective function such that $f\left(x^{*}\right)<f(\mathbb{X})$, being $\mathbb{X}$ the set of all candidate solutions. It is worth to mention that although uncommon, the optimization can also be defined as a maximization process. Another important aspect to note is that the objective function can be much more than a simple distance measurement, thus more complex tasks can be carried out using an algorithmic approach [Wei09]. We will illustrate that kind of approach, tuning a problem solution for making model output to oscillate in a fixed period in the example section of this work.

There are fundamentally three approaches to define the distance metrics for a model [TKG14]. The first approach is based on using acceptable ranges for the model outputs being the most straightforward one. That approach is also known as categorical calibration and works defining intervals for the model output values and when the output falls inside the interval it is considered as having a good fit. One of the main drawback of this approach is the fact that it is not possible to determine how close 
are the model and the reference data. The second metric relies on measuring the differences between simulated and observed values, being the least squares the most commonly used method for computing the quality of fit [BA77]. Finally, that last approach requires the use of likelihood functions. It is hard to implement and it requires that the underlying distribution must be known, which usually precludes its application on complex non-linear computer models.

The systematic exploration of solution space which is compulsory for the calibration process requires many model executions as well as many evaluations of goodness of fit function over the output data to find the best estimation for the model parameters provided that they minimize the discrepancies between simulated and observed values. This is a computationally expensive task, especially in the case of Individual-based models, as the problem bounds increases with model complexity and the number of input parameters which must be tested. Roughly speaking there are basically two different approaches for generating the sampling points required for estimating parameters. The first of them is based on the definition of sampling schemes such as Monte Carlo sampling, Factorial designs or the Latin Hypercube sampling. These techniques work by generating an a priori set of samples in the search space, that is to say, a collection of parameter combinations which are further used for running model and evaluating the cost function [TKG14] [Via13]. The Latin hypercube sampling is a generalization of the Latin squares classical experimentation design randomization typically found on agricultural experimentation [LH78].

On the other hand, in the case of using optimization methods, only an initial set of points, sampled from the input space are instantiated and these solutions are updated dynamically searching for neighboring solutions which could approximate better towards to the minima. The exact implementation details, depends on the metaheuristic chosen for the parameter estimation process, but despite of the diversity of existing metaheuristics practically all of them can be functionally described and completely characterized by combining the building blocks contained in the pseudocode shown in Algorithm 5.1.

Despite of the multiplicity of existing metaphors inspired on a many different sources, ranging from physics or the collective behavior of some eusocial insects to the musical theory [Sör15], most of them are just slight variations over the basic evolutionary strategy skeleton. As can be seen in Algorithm 5.1 the metaheuristic structure contains 


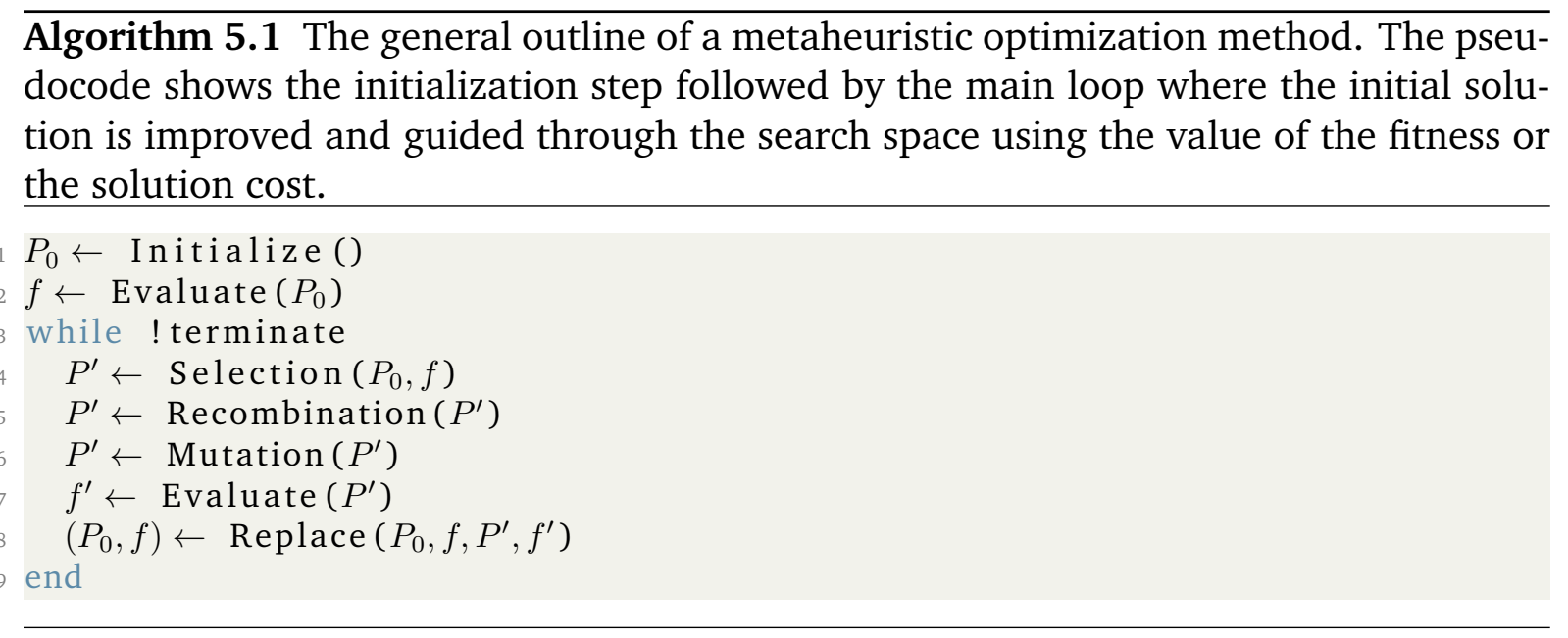

few operators depicted in the algorithm by the functions Selection(), Recombination(), Mutation() and Replace() the other components are the Initialization() and the Evaluate() function. The selection function is responsible for picking parents from the current population which will be used later for producing the offspring in the next generation. The selection process can be stochastic or using the fitness metric for selecting breeding individuals. The objective of recombination process is to mimic, in some extent, the genetic chromosomic recombination and mix together $n$, being $n>1$ parent solutions for producing an offspring which will be the combination of the solution structure of $n$ parents. The mutation operator, roughly speaking, is to generate stochastically random changes in the solution structure providing the necessary variability for exploring the problem space. Finally, the replacement process will select the individuals based on their fitness values from the current solution which will be conserved in the next algorithm iteration returning a tuple $\left(P_{0}, f\right)$ with the individual solutions and its associated fitness. The accessory functions initialize and evaluate are required respectively for instantiating the initial solution and for evaluating the fitness metric for the provided solution set $\mathbb{S}$. With respect to the termination condition for the algorithm, the most commonly used approach is a combination of the convergence criteria and the maximum number of iterations. It is worth to say that not necessarily all these components are required to be present on specific metaheuristics. For instance, the simulated annealing [KGV83] uses a population of size equal to one, therefore the selection and recombination process are superfluous in this metaheuristic. 


\subsection{Metaheuristics for Parameter Estimation}

In order to facilitate the parameter estimation task of Individual-based models we introduce the GNU R [R C15] package EvoPER - Evolutionary Parameter Estimation for Repast, an open source project intended to facilitate the adoption and application of evolutionary optimization methods and algorithms to the parameter estimation of IBMs developed using the Repast Symphony framework [ $\left.\mathrm{NCO}^{+13 a}\right]$. The EvoPER package is released under the MIT license being the binaries available for download from CRAN (https://cran.r-project.org/web/packages/evoper/) and the complete source code for the project can be found on GitHub (https://github.com/ antonio-pgarcia/evoper).

The package EvoPER provides implementations of most common and successful metaheuristics algorithms for optimization specially crafted for searching the best combination of input parameters for Individual-based models developed in Repast Simphony. Current version of EvoPER package supports the Particle Swarm Optimization (PSO) [KE95], the Simulated Annealing (SA) [KGV83] and the Ant Colony Optimization (ACO) [DBS06] algorithms for parameter estimation. We also plan to support more algorithms in successive versions. The metaheuristic algorithms use bio-inspired, natural or physical system analogy having each of them subtleties making them suitable for different types of problems. Nonetheless, despite of the differences in the chosen natural metaphor all algorithms share an important aspect which is that the search space is traversed downhill but allowing uphill moves to avoid to get trapped in a local optimum far from the global one.

The basic PSO algorithm uses the idea of particles moving in a multidimensional search space being the direction controlled by the velocity. The velocity has two components, one towards to the direction of best value of particle $p_{i}$ and other towards to the best value found in the neighborhood of particle $p_{i}$ [KE95] [PKB07]. The behavior and convergence of the algorithm is controlled by the particle population size and by the $\phi_{1}, \phi_{2}$ parameters which respectively controls the particle acceleration towards the local and the neighbor best. The algorithm implementation and the default values for the algorithm parameters follows the guidelines and standard values for the algorithm parameters facilitated by [Cle12] which are proved to provide the best results.

The metaheuristic known as Simulated Annealing uses the idea of a cooling 
scheme to control how the problem solutions are searched. The algorithm generates an initial solution and then iterates, looking for neighbor solutions, accepting new solutions when they are better than the current solution or with some probability $P$ which is function of current temperature and the cost of solutions. Important parameters are the initial temperature $T_{0}$, the final temperature and the cooling scheme [KGV83]. In the implementation, available on the EvoPER package, the default function for temperature update is $T=\alpha T$, being $\alpha$ the parameter controlling how fast the temperature is decremented. In addition, there are other methods readily available on the package for cooling and the users can also provide their own temperature decrement function.

The Ant Colony Optimization algorithm is settled over the computational metaphor of the stigmergy mechanism found in ant communities and used by the individuals for coordinating their activities in the search for food. Specifically, in the case of ant foraging behavior, the stigmergy is implemented by the pheromone reinforcing system where the most travelled way becomes the preferred one, owing to the proportional increment of pheromones deposited on the environment [DG97]. The algorithm controls the convergence with the pheromone update and the pheromone evaporation processes. The evaporation avoids the rapid convergence to a local optimum [DBS06]. The standard version of ACO algorithm is well suited to discrete combinatorial problems but its application to continuous problems require some tweaking. Thus, to cope with these limitations an extension generalizing $\mathrm{ACO}$ for continuous domains and denominated $A C O_{\mathbb{R}}$ has been proposed [SD08]. That extension, while keeping the underlying idea, replace the pheromone system by an equivalent structure called solution archive which stores the $s_{l}$ solutions, the results of $f\left(s_{l}\right)$ function evaluations and finally the weight $\omega_{l}$ [SD08], for $i=1, \ldots, k$, where $k$ is an algorithm parameter for configuring the size solution archive. Finally, another component of the algorithm is the Gaussian kernel which is sampled for updating the solutions. The kernel contains $k$ Gaussian functions, one for each row $l$ in the solution archive.

The two metaheuristics introduced in this work, the ees 1 and ees 2 are simple strategies for tackling with the high computational cost of executing complex individualbased models the minimal number of iterations required for ensuring the algorithm has found an acceptable optimum value for the combination of model parameters when there is no information about what are their physically relevant ranges. We had developed the ees 1 and ees 2 for analyzing the parameter space of our own individual-based 
models [PRP15b] [PRP15a] of conjugation plasmid [AF13] dynamics within bacterial colonies. The underlying idea behind these metaheuristics is that we are interested on keeping the track and mapping the visited search space, rather than getting a point estimate for the best value, which may not be completely suitable for individual-based models because of the high stochasticity in the model output response. The obvious alternative for facing with the variability in the model output is increasing the number of replications for each parameter combination, but it would increase the execution time so much, rendering impractical the approach without parallelizing and distributing the load across several computer nodes.

The ees1 metaheuristic introduced in this work stands for EvoPER Evolutionary Strategy 1 and it is an instance of a custom evolutionary strategy which can be described by the commonly used notation as $\left(\mu+\rho_{w} \lambda\right)$-ES being $\mu=\lambda$, which basically means that every generation only the fittest individuals or, some suboptimal individuals selected with a probability $P$ taken from the parents and offspring collection, will become parents for the next generation. The mating selection process implemented in ees 1 chooses half of the existing $\mu$ parents for being used in the recombination round.

The algorithm has a parameter for parent selection which also uses the Greek letter $m u$ but it must not be confounded with term used previously for describing the parent number in the evolutionary strategy descriptive notation. The parents are sorted by their fitness values and they are selected with an exponentially decreasing probability weight which is calculated using the expression $P(\mu)^{k}, \forall k=1 \ldots N$ where $P(\mu)$ is the probability of selecting individuals with a suboptimal fitness, that is to say, when the values of $P(\mu)$ are small the solutions with the best fitness are string preferred and as the value $P(\mu)$ tends to 1 the selection becomes a random process where the individuals are selected using an uniform deviate, see the Figure 5.1 for visualizing how the parameter $\mu$ affects the probability of picking an individual having higher cost values.

The selected parents are used for calculating the geometric average which in turn serves as the centroid measure $\mathcal{C}$ for the recombination process in a sense that all individuals in the current solution population are recombined by calculating the arithmetic mean of the solution value and the $\mathcal{C}$. For executing the recombination process two different approaches are used, one of them is chosen with a probability $P=1 / 5$ and other with $1-P$. The first approach uses a weight value calculated as $w_{k}=f\left(x_{k}\right) / \sum_{k=1}^{N} f\left(x_{k}\right)$ 
Probability $\mathrm{P}(\mathrm{mu}, \mathrm{Si})$

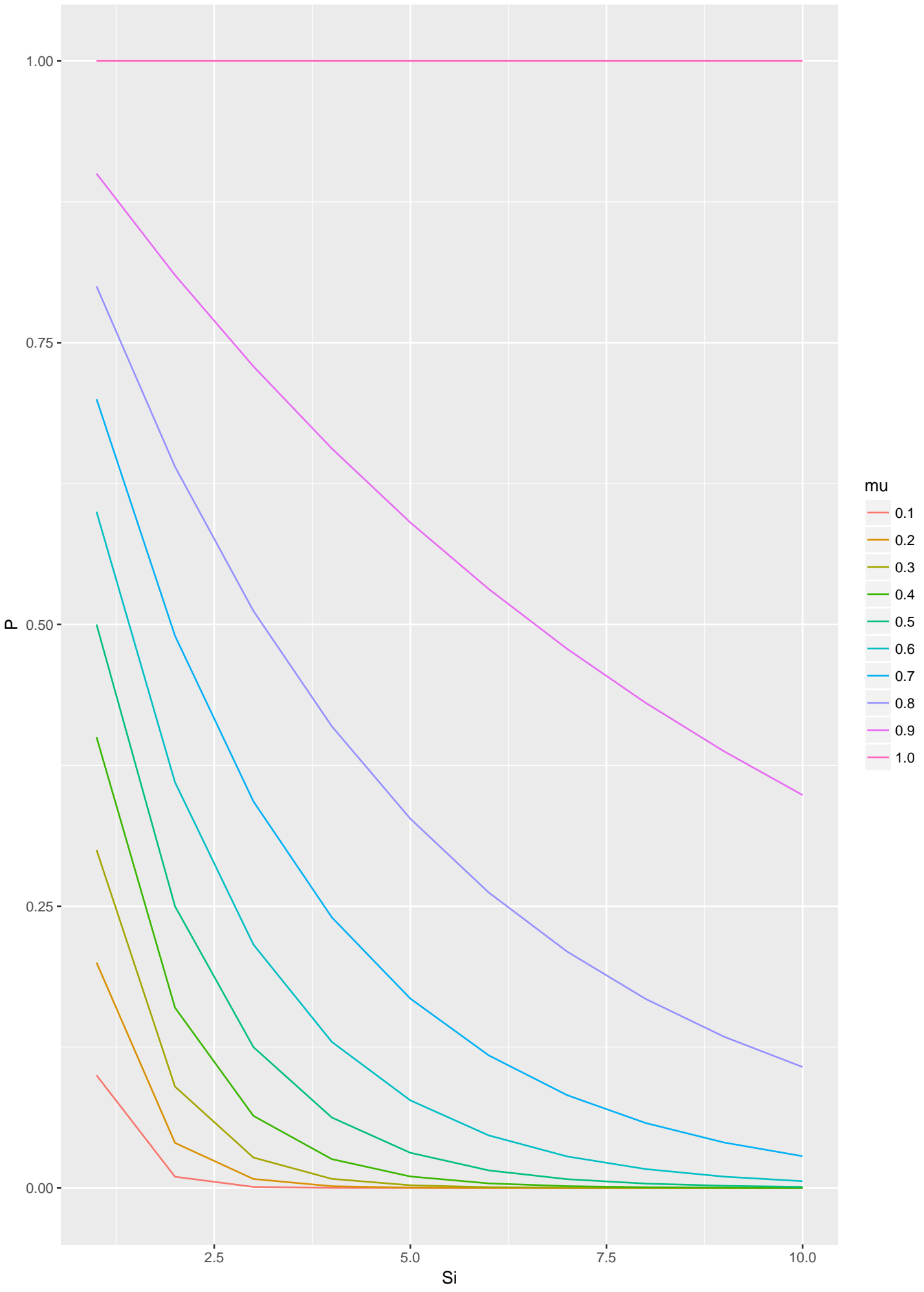

Figure 5.1: The probability $P(\mu)^{k}$ of selecting and individual having a suboptimal fitness value. 
for calculating the recombination component $\mathcal{R}=\left(x_{k}+\mathcal{C}\right) * w$. The new value of $x_{k}$ is the average $\left(x_{k}+\mathcal{R}\right) / 2$. The second approach selected with probability $1-P$ is simply the average of $x_{k}$ and $\mathcal{C}$. More details on the algorithm can be seen in Algorithm 5.2.

The last component of ees 1 metaheuristics is the environmental selection where the fittest individuals are selected for being part of next generation replacing the current solution elements. The best of all individuals from current population is always chosen for participating as a parent in the subsequent algorithm iteration and the remaining population components of suboptimal individuals can be replaced by the new individuals with a worse fitness value with a probability $P(S)$ which represents the strength of selective pressure. Thus, the range of $\kappa$ parameter may vary between $0 \leq \kappa \leq 1$, being the lower bound the absence of selective preference and the upper bound the maximum selective pressure over the population. In other words, a value of $\kappa=0$ means all elements but the best of current population are replaced by the offspring independently of their fitness. On the other hand, a value of $\kappa=1$ implies that only individuals improving the fitness of current solution are picked up for becoming part of next generation. For other values in the $\kappa$ parameter range, the selection process execute downhill movements with a probability $P(1-\kappa)$.

The second algorithm ees 2 is a very simple metaheuristic which is based on gradually reducing the initial dimensions of search space. The algorithm works by taking samples points of parameter space using Latin hypercube sampling scheme and allowing them to vary over their full range. Thus, for each iteration step, the initial set of problem solution points are further refined using the defined fitness metric as guidance for reducing the full span of variation of each of the analyzed input factors. The parameters of this algorithm are the population size $N$, and the fraction of population size $\rho$ which will be used for calculating the new ranges for model parameters. The default values of these parameters are $N=100$ and $\rho=0.05$ but it can be tweaked depending on the number of input parameters being estimated.

The Algorithm 5.3 shows the general outline for the algorithm. As can be seen, the initialization section generates a sampling of problem space using Latin hypercube using the initial vector of input parameters $\vec{p}$ and the population size $N$. The input parameter vector $\vec{p}$ contains tuples with the range of values for input factors. Once initialized, the fitness function is evaluated and the results are added to solution $S$ and sorted using as sort key the fitness value. Thus, the best input parameter combinations 


\begin{abstract}
Algorithm 5.2 . The pseudocode of evolutionary strategy 1 ees1 . The algorithm encompasses the standard components of an evolutionary strategy. First, the initial set of solutions is instantiated and evaluated. Subsequently, inside the main loop N/2 individuals are selected with a probability $\mathrm{P}$, for estimating the geometric mean which will be employed for being recombined with the current solution population using a fitness weighted arithmetic mean. The next step is the mutation process, consisting in making random changes in solution components with a probability $\rho$ from 1 to $p$ variables, being $p$ the number of model parameters. Finally, if new solution improves the fitness, the current best solution is updated. The current best population solution
\end{abstract} $S_{i}$, for $i>1$, is also updated with worst solutions with a probability $\kappa$.

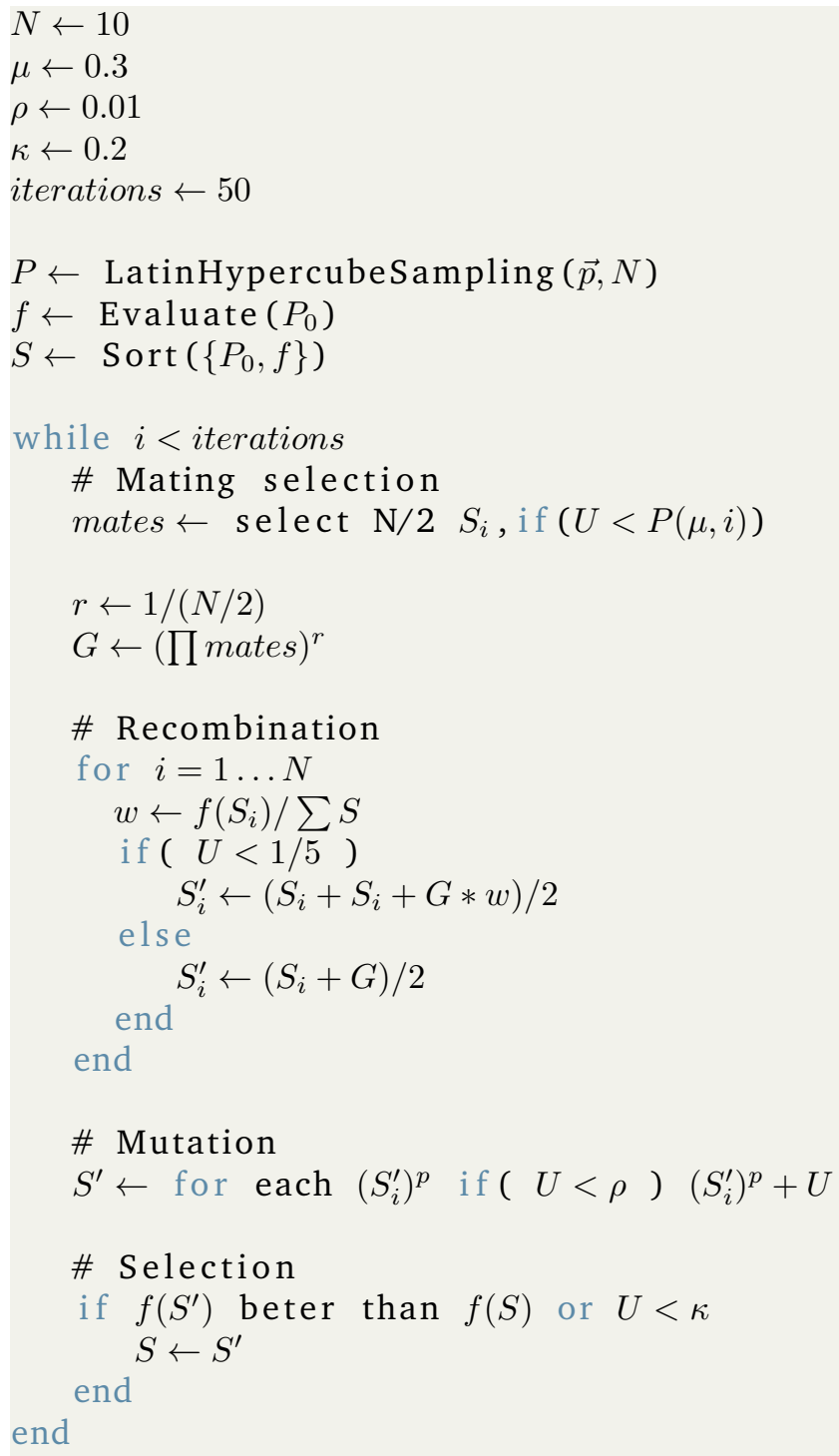




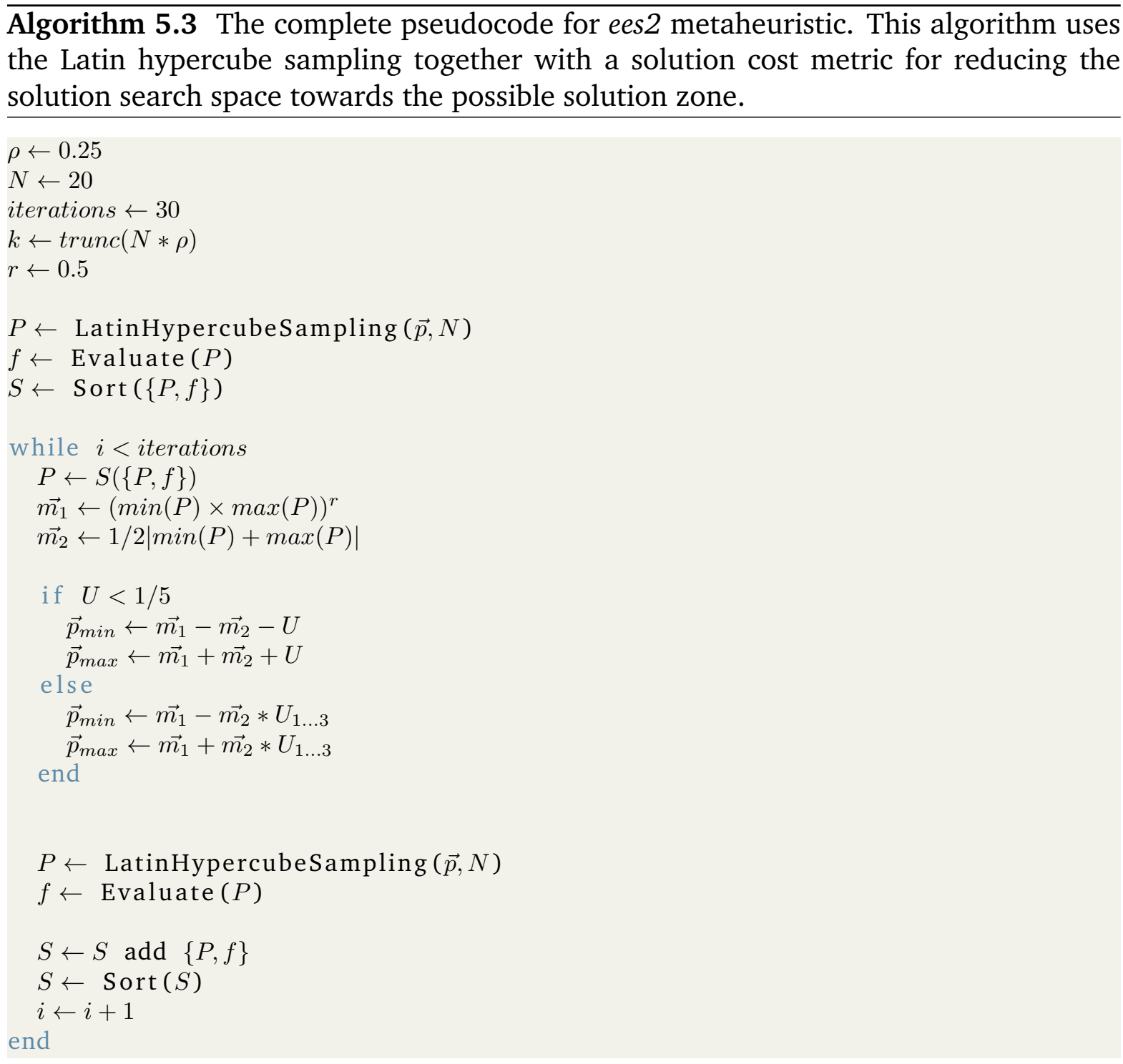

for the problem are on the initial rows of $S$. The solution $S$ is a matrix with $m=N$ rows and $n=|\mathbb{X}|+1$, being $N$ the parameter population size and $\mathbb{X}$ the set of input parameters, hence every row of $S$ have an instance problem input parameters and the fitness value for that parameter combination. The next section is the algorithm main loop where the search region is updated every iteration considering the first $k$ values from solution matrix. The number $k$ is calculated using the parameter $\rho$ and usually should be between $5-10 \%$ of population size $N$. With the subset of solution matrix, the 
value of interval $I$ is calculated as the arithmetic average for the minimum maximum values of first $k$ elements of solution matrix $S$. The minimum and maximum values of parameter range are then calculated using the average of first $k$ values of solution matrix $S$, the interval and a small random perturbation, then the new vector of input parameters $\vec{p}$ is used for generating the new round of sampling points using the Latin hypercube. The new population is evaluated and combined with the current solution $S$. The new solution is sorted and a new iteration takes place using again the first $k$ values from solution matrix. As can be observed the algorithm is pretty simple but effective for mapping promising zones of solution space with a relative few number of iterations. It has not been extensively tested yet but when applied to standard optimization problems produce consistent results.

The package was designed following an object-oriented approach, being structured around an entry point function and a class hierarchy representing an abstraction for the objective function to be optimized and by class encapsulating return type for the optimization methods. The classes abstracting the objective function are the basic input for the optimization algorithms available on the EvoPER package and can be extended for supporting other simulation platforms.

There is a parent class called ObjectiveFunction with two subclasses, namely the PlainFunction and the RepastFunction. The purpose of the first subclass is to allow the user to run the optimization algorithms using their own mathematical functions which can be useful for testing purposes or for wrapping other types of simulation subsystems. The second subclass encapsulates the Repast model calls for executing the chosen optimization algorithm for estimating the model parameters. The entry point function returns an object instance of Estimates class. A brief description of package with the partial structure of EvoPER classes for encapsulating the target of parameter estimation and the main methods is given in Boxes shown below but for a complete and updated reference please refer to the package manual.

\section{ObjectiveFunction}

The base class in hierarchy providing the skeleton for running the optimization algorithms. 


\section{ObjectiveFunction,Parameter}

The objective of this method is adding a parameter with range between a minimum and a maximum value. The parameters must coincide with those defined in the model.

\section{ObjectiveFunction,getParameter}

Returns a previously defined parameter. The companion method getParameterNames returns a list with the names of all user defined parameters.

\section{ObjectiveFunction,Evaluate}

The Evaluate method is responsible for wrapping the objective function evaluation and must be overridden by all those classes extending the parent class ObjectiveFunction.

\section{ObjectiveFunction,EvalFitness}

Executes the objective function and returns the results. The user code must prefer this method for executing the objective function.

\section{ObjectiveFunction,RawData}

Returns the complete raw output of objective function whenever it is available.

\section{ObjectiveFunction,stats}

Provides some basic statistics for the ObjectiveFunction execution.

\section{PlainFunction}

Allows the optimization of plain functions implemented in $\mathrm{R}$. 


\section{PlainFunction, initialize}

The initialize method must be overridden in subclasses of ObjectiveFunction and it is responsible for bootstrapping the real implementation of target function. In the case of Plainfunction it requires any user provided $R$ function as parameter.

\section{PlainFunction,Evaluate}

Overrides superclass method with the specific function call.

\section{RepastFunction}

Wrapper for the Repast Model objective function.

\section{RepastFunction,initialize}

This method is a wrapper initializing the Repast Model constructor. Requires the model directory, an aggregated data source, the total simulation time and a user defined cost function.

\section{RepastFunction,Evaluate}

Overrides superclass method with the specific function call. 


\section{Estimates}

The Estimates class serves as the standardized return type for all optimization methods available on the package. The initialized instance of this class stores the values of best value ever found during the metaheuristic execution, the list of best values for every algorithm iteration and finally the complete collection of all points which have been visited in the solution space which can be particularly useful for mapping the promising zones solution space.

\section{Estimates,getBest}

This method returns the best value ever found for the objective function.

\section{Estimates,getIterationBest}

Returns a list with the best values found for every iteration.

\section{Estimates,getVisitedSpace}

The method returns a collection which contains the results of all evaluations of the objective function.

The object-oriented approach allows the easy extension of the package for other types of Individual-based modeling tools or methods. As can be seen in the previous boxes with the classes and methods, the only requirement to apply the methods contained in the EvoPER package is to extend the ObjectiveFunction class and override the Evaluate method to support the new parameter estimation target. One of the useful aspects of EvoPER implementation is the possibility to specify constraints in the search space by individually setting lower and upper bounds for every parameter being analyzed using the ObjectiveFunction\$Parameter(name, min, max) method. That is an important point for limiting the parameter values only to the acceptable biological range.

The workflow for carrying out the parameter estimation consists in a simple sequence of steps. First, an object instance of any ObjectiveFunction subclasses must 
be created and properly initialized. As mentioned previously, currently we have two options available for parameter estimation: one for simple functions which could be used for testing purposes (PlainFunction) and another for estimating parameters of Repast models (RepastFunction). Once the objective function has been initialized, the required parameters must be provided with the appropriate lower and upper bounds. Finally, the extremize function can be applied to the previously defined function. The required parameters are the optimization method and the objective function instance. The function has a third optional parameter for providing the custom options for the underlying optimization method.

The overview of the most relevant EvoPER API functions. The current a list with implementation of metaheuristic methods for parameter estimation. The optimization functions available are shown in the Boxes presented below for providing an overview on the package contents. The EvoPER package is still in and early phase of development therefore the list could change over the time. The package manual will be the most updated source of information for the package contents.

\section{extremize}

This is the entry point function for all available parameter estimation methods and should be preferred instead of direct call to the underlying functions. It has three parameters, being the first two of them required and the third optional. The first parameter is a string indicating the metaheuristic algorithm, currently accepted values are ("pso"|" saa"|" acor"|"ees1"|"ees2") for particle swarm, simulated annealing, ant colony respectively, the evolutionary strategy 1 and evolutionary strategy 2. The next version will include also genetic algorithms (GA) and Tabu Search (TS). The second is an instance of the objective function and finally, the third one is an instance of a subclass of the Options class specific for the selected algorithm. If not provided the default options for the metaheuristic will be used. The extremize returns an initialized object instance of Estimates class containing the results for the optimization method. 


\section{abm.acor}

The abm.acor implements the Ant Colony Optimization for continuous domains. The function requires an instance of a subclass of ObjectiveFunction and an optional parameter with an instance of OptionsACOR. Currently there are two subclasses of ObjectiveFunction, one for optimizing plain $\mathrm{R}$ functions (PlainFunction) and another for Repast Models (RepastFunction).

\section{abm.pso}

The function call for running the Particle Swarm Optimization method. It is necessary to provide a subclass of the ObjectiveFunction and optionally an instance of OptionsACOR. If not options are given a default instance will be used for the maximum iterations, the swarm size, the acceleration coefficients, the inertia weight or constriction coefficient and finally the neighborhood type.

\section{abm.saa}

This is the implementation of Simulated Annealing algorithm and identically as in the previous cases the function requires an instance of the objective function and accepts an instance of OptionsSAA. The options class have acceptable default values for the initial temperature, the minimum temperature, the temperature length, the cooling ratio, the neighborhood distance as a fraction of parameter range and the neighborhood function. 


\section{abm.ees1}

The EvoPER Evolutionary Strategy 1 (ees1) is a simple evolutionary strategy which uses the geometric mean as the focal point for constructing the recombination model for the next generation of candidate solutions. The metaheuristic allows the configuration of several parameters, namely the solution size $(N)$, the mating selection strength $(\mu)$, the mutation rate $r h o$ and the selective pressure $(\kappa)$. The default values can be changed by providing an instance of options class OptionsEES1 with the desired values.

\section{abm.ees2}

That is not exactly an evolutionary strategy stricto sensu because the new generation solution is not created directly from parent solution but instead, parents are used for searching the new range of solution parameter space. The algorithm is based on reducing the initial parameter space and generating new solutions with the new ranges for each iteration. The solutions are generated using the Latin hypercube sampling scheme. The configurable parameters are basically the population size $N$, the number of algorithm iterations and the selection ratio $\rho$ which allows the specification of a fraction of $N$ for estimating the new boundaries. The default values for both parameters are 10 and 100 respectively. For modifying these settings, it is necessary to provide an instance of options class OptionsEES2 with the desired values. It is intended to provide an acceptable approximate for parameter estimation in fewer model executions.

The particle swarm optimization metaheuristic implementation requires a topological neighborhood function which provides the structure for the swarm particles allowing the algorithm to select the best position in the solution search space. The package provides three different implementations for the neighborhood selection: pso.neighborhood.K2, pso.neighborhood.K4 and pso.neighborhood.KN. The first topology function returns two neighbors of solution particle $x_{i}$, where the neighbors are the particles $x_{i-1}$ and $x_{i+1}$ using a ring topology [ZBCR13]. The second function returns four neighbors of particle $x_{i}$ using a von Neumann neighborhood function. Finally, the last function returns a complete graph with the whole set of particles. The 
default implementation uses the entire population as the neighborhood. In addition to these functions, it is also possible to provide a user defined neighborhood function creating a non-default instance of the OptionsPSO class and passing the reference to the alternative implementation using the method neighborhoodFunction() of Options class. The neighborhood function signature is shown in Figure 5.2. The function is invoked inside the PSO code which passes the position of current particle $i$ and the size of particle population $n$; the function must return a collection of integers with the neighbor positions, grouped with the $\mathrm{R} c()$ call.

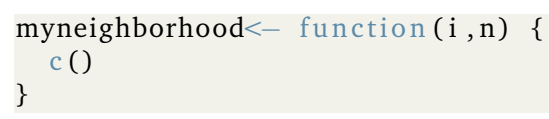

Figure 5.2: The function signature for the custom particle swarm neighborhood. The function can return any number of neighbors from $i$ to $n$ and the returned values must not be greater than $n$.

Most of the aspects implemented in the optimization code are standard and, perhaps the only points which are specific to the EvoPER package, are the neighborhood function for pso.neighborhood.K4 and saa.neighborhood. The von Neumann neighborhood for particle swarm optimization is generated using a topology created converting the linear collections of particles to a matrix using the $R$ code $\mathrm{m}<-\operatorname{matrix}(\operatorname{seq}(1, N)$, nrow $=(\operatorname{ceiling}(\operatorname{sqrt}(N))))$ where $N$ is the swarm size.

The case of generating the neighborhood solutions for simulated annealing has been attacked using the following logic for generating new solutions: first pick randomly the parameters to be perturbed ${ }^{1}$ and update them using two different paths selected randomly, preferring the second with a probability $P=0.8$. The first of them is based on drawing a number from a normal deviation given by the expression $S^{\prime}=\Delta Z+$ $\bar{S}$ where $S^{\prime}$ represents the new solution, $\Delta$ the standard deviation calculated as the range of parameter $p_{k}$ multiplied by the algorithm parameter distance $d=0.5$ (default value) finally, the $\bar{S}$ is the arithmetic average between the minimum and maximum allowed values of parameter $p_{k}$. The second one uses the expression $S^{\prime}=S+S *$ $U(-1,1)$ where $S^{\prime}, S, U$ are respectively the new neighbor solution, the current solution,

\footnotetext{
${ }^{1}$ The neighborhood functions currently implemented allows choosing from $1,1 / 2 \mathrm{n}$ or $\mathrm{n}$, being $\mathrm{n}$ the number of parameters which will be perturbed. The implementation can be easily extended for accommodating any user defined neighborhood algorithm.
} 
a uniform random number between $[-1,1]$.

The simulated annealing algorithm also needs a function for generating other points in the solution space close to the actual current solution. The currently available neighborhood functions for perturbing the best solution are: saa.neighborhood1, saa.neighborhoodH and saa.neighborhoodN. The difference between these implementations is basically the number of problem dimensions to be perturbed. Thus, the first function alters just one element of the current solution, the second changes half of the solution elements at a time and finally the last one modifis all dimensions of a solution. The solution components to be perturbed are chosen randomly. Again, it is possible for the package users to provide their own implementation for the neighborhood function.

The package provides acceptable default values for most of parameters related to the optimization method in use. In spite of the fact that the parameter estimation functions can be called directly, the users should use the function extremize $(m, f, o)$ which is the standard entry point for the optimization methods. As has been mentioned previously, the function has three parameters, which are respectively the method $(m)$, the objective function $(f)$ and the options $(o)$. Only the first two are required and the third is optional. When the options parameter is not provided the default values are used. If settings different from those default values are required, the user must pass an instance of the corresponding option class. For example, if more iterations are required for PSO method an instance of OptionsPSO must be created and the method setValue ("iterations", value) with the appropriate value. Many other parameters can be customized in order to fit the specific needs for the model being analyzed such as the neighborhood functions or the temperature update for the simulated annealing.

\subsection{Discussion}

In this section, we will show some small and illustrative examples about how to use the EvoPER package for estimating the parameters of different kinds of models. The first example includes the parameter estimation required minimizing the standard functions employed for testing optimization methods. The second example shows show to adjusting the output of a real individual-based model to match the experimental data. The next one is about how to tune the model output for oscillating with any specific 
user defined period. Finally, the last one gives an example on how to explore the parameter solution space for getting a landscape with suitable solutions for the problem being addressed.

\subsubsection{Optimizing simple functions}

It is worth mentioning that although the package is oriented to the application of evolutionary optimization methods to the parameter estimation of models developed using Repast Simphony it can also be used to minimize mathematical functions as well as, extended for other individual-based modeling frameworks. In the following example shown in Figure 6.5 we demonstrate the package usage applying it to the two variables Rosenbrock's function.

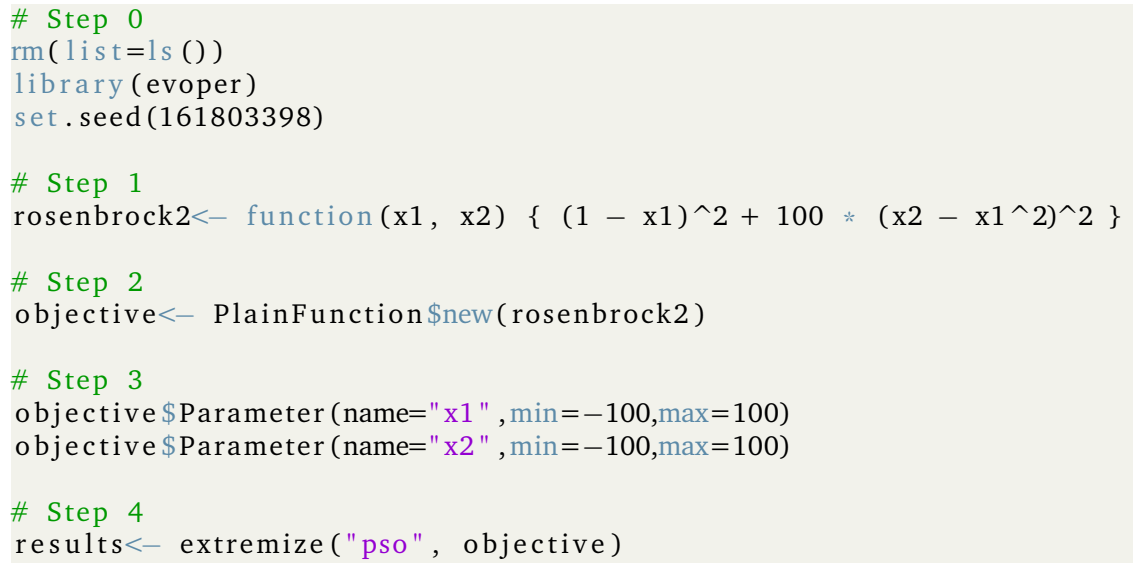

Figure 5.3: A simple example for minimizing the Rosenbrock's function using the EvoPER package.

As can be seen in Figure 6.5 the step 1 shows the definition of a simple function to be minimized; the step 2 demonstrates how to create an instance of PlainFunction class; in the step 3 the parameter ranges for each function's parameter are provided and finally in the step 4 the EvoPER extremize function is used to minimize the objective function. The results of running the example are shown in Figure 5.4 where we can see the estimated parameters, the value of fitness function, the execution time and the number of times the function has been evaluated. 


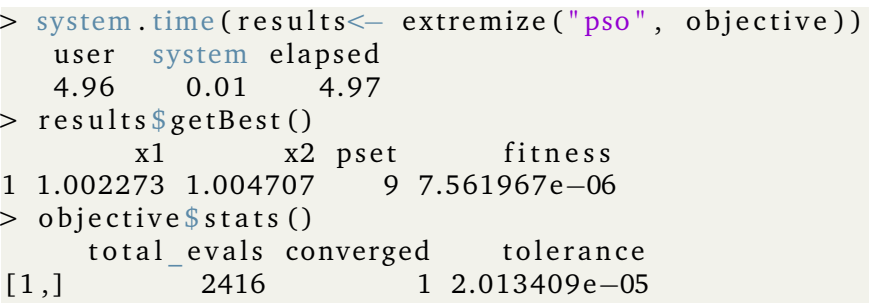

Figure 5.4: The $R$ console output session showing the results of running the previous example.

\subsubsection{Tuning oscillations}

The oscillatory behavior is a structural component of many types of systems requiring timers for controlling and coordinating its processes. It is an integral part of several types of systems, ranging from electronic to ecological or biological processes which normally relies on circadian clocks for regulating faithfully all their internal activities and interactions with environment. Therefore, the design of synthetic biological oscillatory circuits is an important research subject and tuning these circuits for oscillating with a precise period is a cumbersome and trial and error activity [KC10]. Fortunately, tuning the model parameters for finding the desired oscillatory behavior can be expressed as an optimization problem which can be solved using evolutionary algorithms. It is worth to mention that the parameters estimated for any model are just the starting point for the wet-lab work, because the reality is extremely stubborn insisting in not working in line with the values estimated by the model.

For illustrating how to turn that problem into an optimization problem we introduce an example shown in Figure 6.11 where the cost function is crafted for tuning the model parameters in order to accomplish a specific output. Specifically, it is a simple toy model representing the Lotka-Volterra ordinary differential equation system, also known predator-prey is presented and we want to estimate the parameters required to make the output oscillate with a specific period. The model, despite of being developed for modeling the predator and prey relationship, has a broad range of applications and can be used for representing many types of ecological and biological interactions [Sho08]. Additionally, the standard model can be extended for supporting $N$ species interactions. 
Table 5.1: The results of applying particle swarm optimization metaheuristic for period tuning. The table shows the optimum parameter values for the oscillation period.

\begin{tabular}{lllllr}
\hline Period & c1 & c2 & c3 & c4 & cost \\
\hline 12 & 1.798102 & 1.618035 & 1.192361 & 1.453045 & 0 \\
24 & 0.675586 & 1.375913 & 1.169076 & 0.8311187 & 0.04166667 \\
48 & 0.4558475 & 0.4602389 & 1.192546 & 0.5483637 & 0 \\
72 & 0.3297914 & 0.4675479 & 1.650108 & 0.778639 & 0 \\
\hline
\end{tabular}

The standard predator-prey model has four parameters which are necessary to estimate as can be seen in Equation (6.8),

$$
\begin{aligned}
& \frac{d x}{d t}=c_{1} x-c_{3} x y \\
& \frac{d y}{d t}=-c_{2} y+c_{4} x y .
\end{aligned}
$$

where the terms $c 1, c 2, c 3$ and $c 4$ which represent respectively the growth rate of prey, the predation rate, the predation effect on predator growth rate and finally the death rate of predator. The session output is of a model execution for tuning an oscillation period of 72 time units is presented in Figure 5.6 where the values for the parameters required to produce oscillations with the desired period are shown. Additionally, the Table 5.1 shows the complete results for all periods for which the model parameters have been estimated. The Figure 5.7 shows graphically the results for the tuning the model parameters for producing an oscillatory behavior with approximate periods of 12, 24, 48 and 72 time units. The example uses the particle swarm optimization metaheuristic for finding the parameter combination required for making the model produce oscillations. As can be observed, the objective function of metaheuristic can be tweaked for generating any desired output behavior. The most common one is to assess the quality of fit between simulated and experimental data but it is not limited and can be used to find parameter combinations which generate practically any global behavior. 


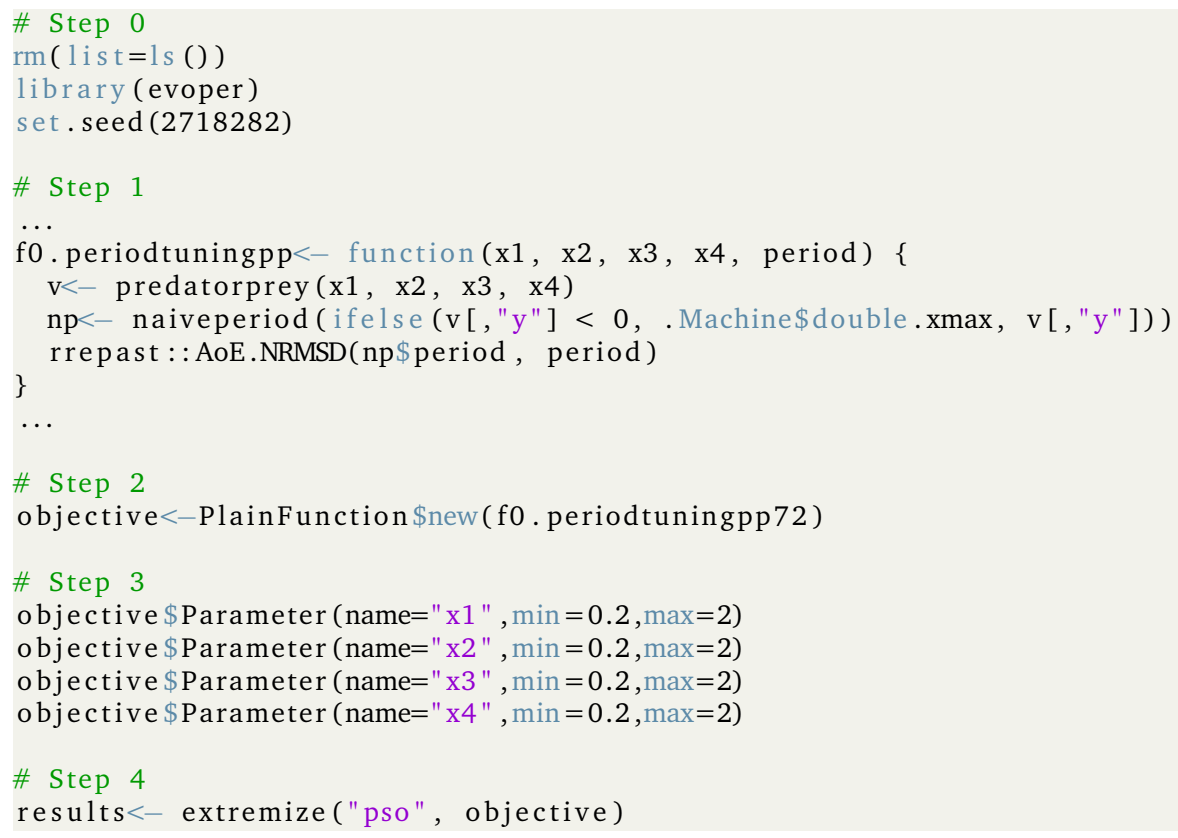

Figure 5.5: Tuning the oscillation period of predator-prey model. The listing has five sections identified by the tags Step 0 to Step 4. The first section consists in loading the library and setting the random seed. The next section is where the cost function is defined, consisting in solving the initial value problem with provided parameters and using the results of ODE for feeding the function named naiveperiod for finding the periods in the differential equation output which is later compared with the reference period using a normalized root mean square deviation (AoE.NRMSD). The subsequent sections encompasses: the initialization of function to be optimized which is a wrapper for the previously defined cost function; the definition of range of variation for the model parameters and finally the application of the metaheuristic with the extremized function call.

\subsubsection{Exploring the solution space}

The particularities of individual-based models which make them so appealing for modeling populations and ecosystems, such as the structural realism, the predictive power, the individual level stochasticity and the emergence of complex global dynamics from the elemental interactions [GAR16] also imply that parameter estimation becomes a complicated matter even when using approximated techniques as those presented in this work. Even simple models contain many levels of uncertainty and certainly presenting nonlinear behavior and possibly discontinuities consequently it is very com- 


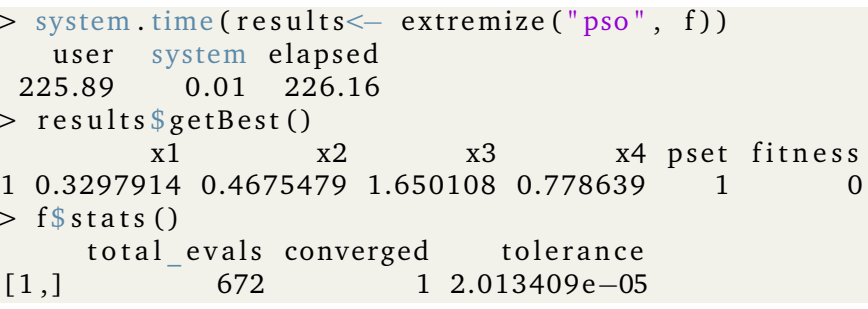

Figure 5.6: The $\mathrm{R}$ console output session showing the results of running predatorprey model in Figure 6.11 .

plicated, using a computationally tractable number of model executions, to make sure that the solution converges successfully to an optimum which is close to the better solution. Normally, modelers have not enough information about all model parameters and it is no uncommon to make assumptions or educated guesses for the acceptable ranges considering the physical or biological constraints. Of course, it is not a random choice but it is far from being a perfect process and, despite of guessed parameter ranges are hopefully within the same order of magnitude of their real values, usually they may diverge by a factor of two or three [MPN16] which may produce odd results when adjusting several parameters.

It is worth noting that it should not be expected a perfect match between the model predictions and the experimental data, consequently it is very unlikely that optimization algorithms converge using the tolerance levels used normally for numerical optimization of plain functions. One way to tackle with this situation is defining the objective function for the parameter estimation using a categorical approach with a not very strict range of acceptance but that may lead to loosing information which may be relevant and giving the false feelling that the right parameter combination has been found. That is a serious issue which may render impossible to draw any conclusion from the parameter estimation results. A possible alternative is not relying exclusively on the best value ever found for the cost function but instead, leveraging the intermediate results for analyzing the problem using it for building a landscape of solution space. The metaheuristics described in this work have a slight modification for saving the partial best results for every interaction and the complete set of points visited in the problem solution space which are made available as two methods of Estimates class, respectively getIterationBest() and getVisitedSpace(). Using these two methods the solution space 
(a) pso, period: 12 time units

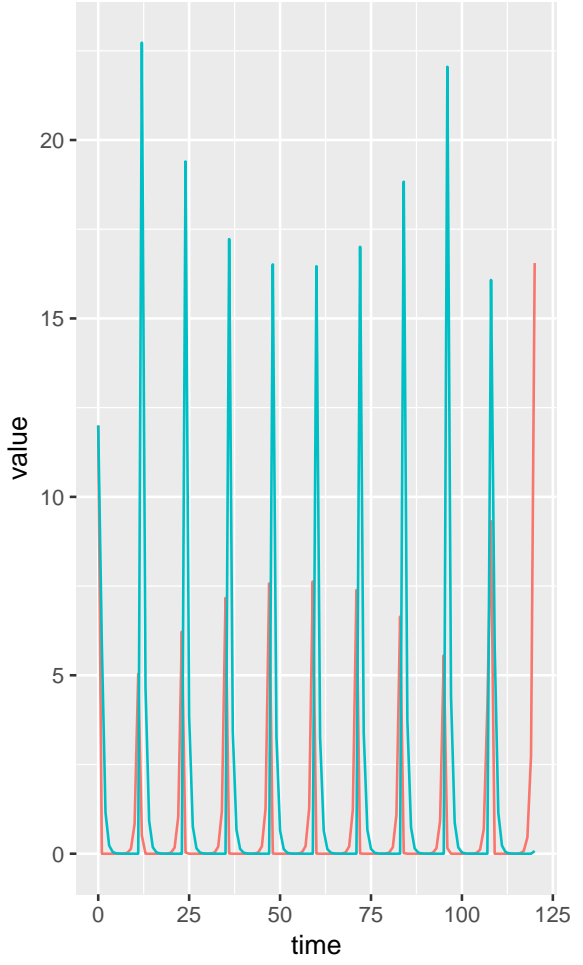

(c) pso, period: 48 time units

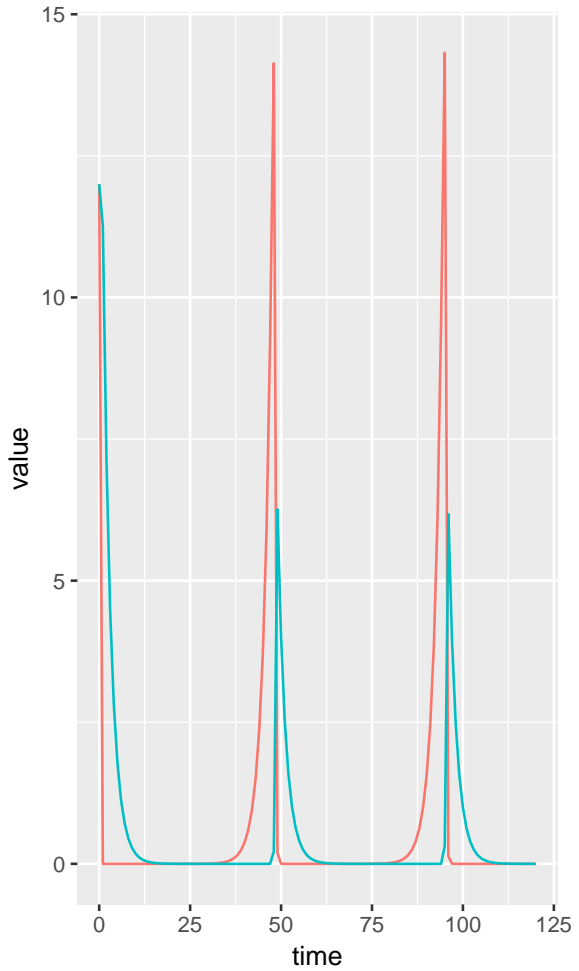

(b) pso, period: 24 time units

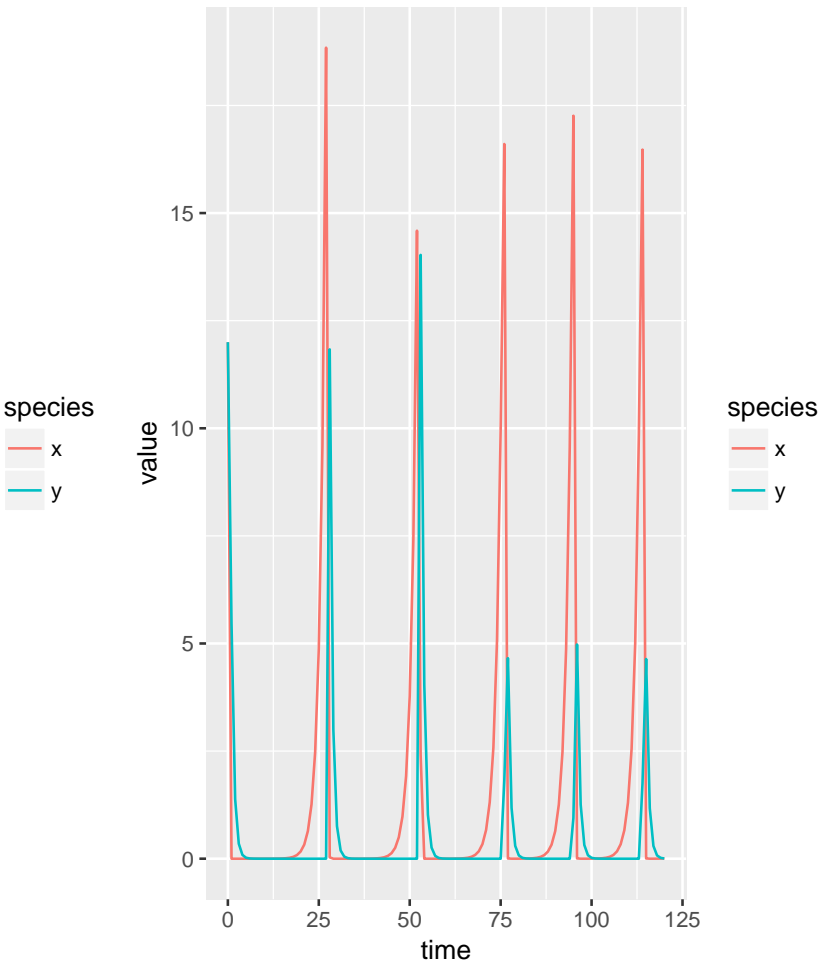

(d) pso, period: 72 time units

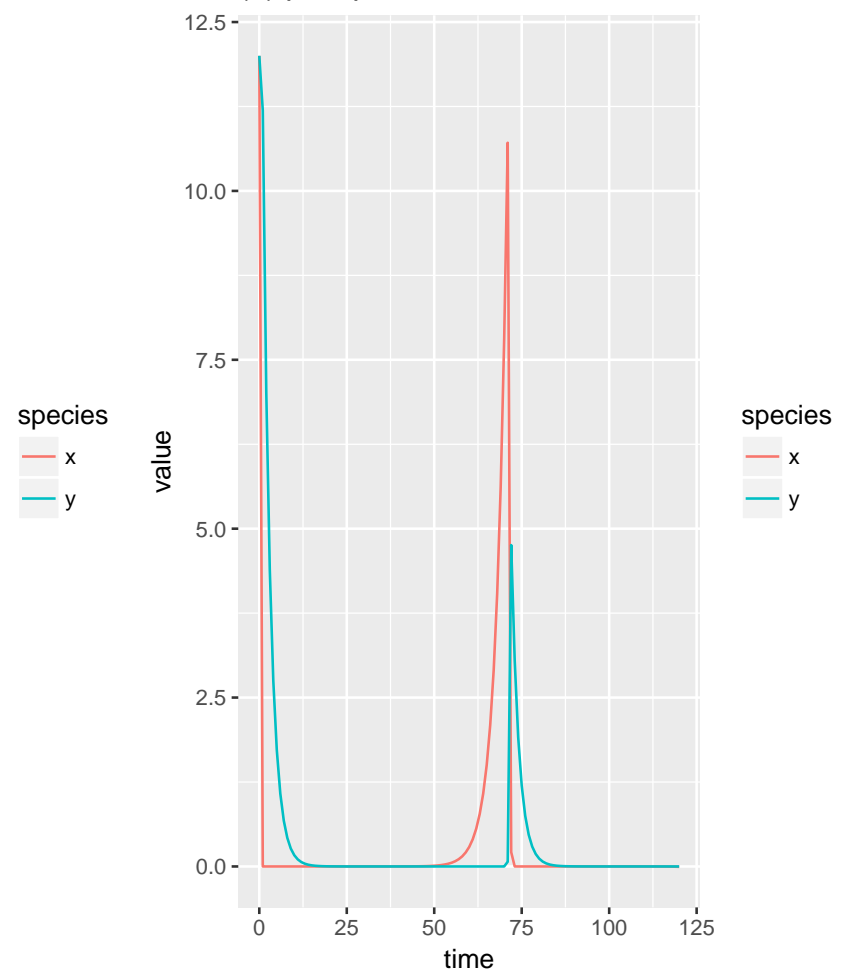

Figure 5.7: An example of tuning the oscillation periods of predator-prey model. In this figure, we can observe how $x$ and $y$ species, respectively the prey and predator components oscillates with different periods. The oscillations with periods of 12, 24, 48 and 72 time units as can be seen respectively in subfigures (a), (b), (c) and (d). 
can be mapped for viewing the most promising zones. The code in Figure 5.8 shows how to generate a contour plot for the solutions space for a four variables instance of Rosenbrock function.

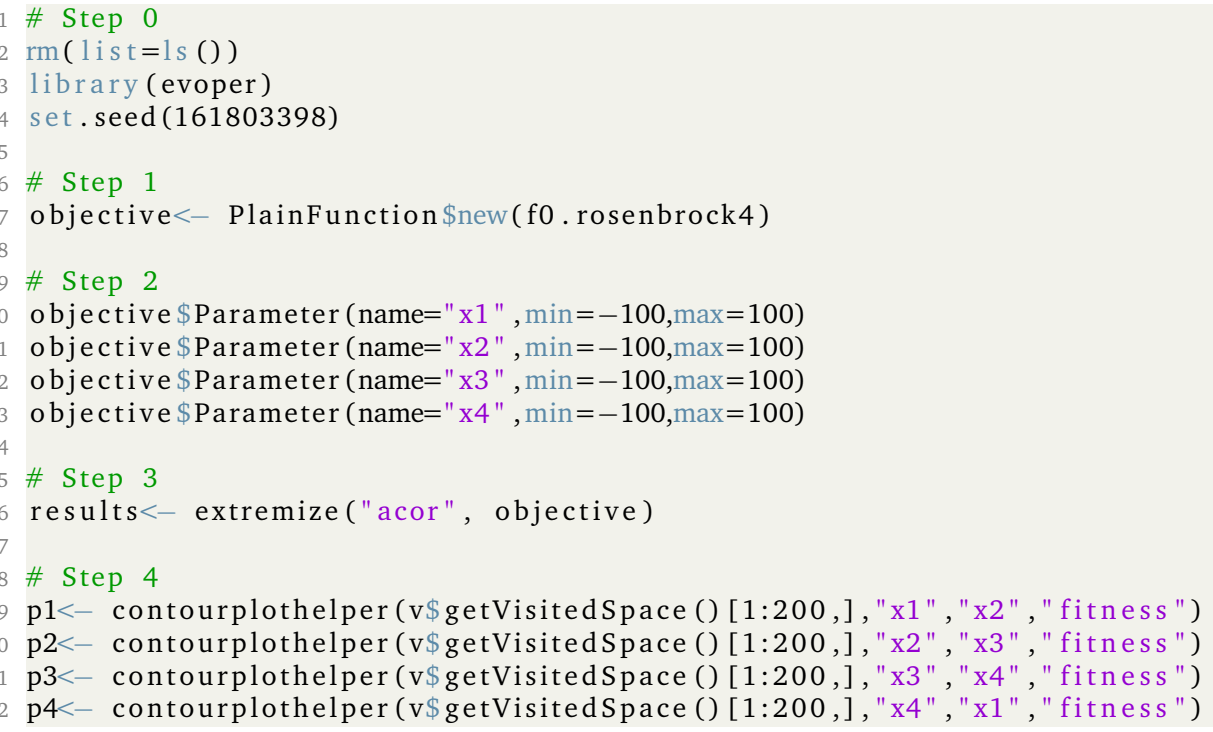

Figure 5.8: Exploring the solution space for Rosenbrock function of four variables using the Ant Colony Optimization for continuous domains (acor) algorithm. The sequence of steps is practically the same presented previously with the exception of Step 4 which show the generation of four contour plots using the first 200 values retuned by the getVisitedSpace() method which are sorted in ascendant order by the fitness value.

The complete plot generated with the fourth step of command sequence provided in 5.8 is shown in Figure 5.9. These contour plots facilitate mapping and visualizing the promising zones of solution space using the fitness of the solution generated as the $z$ value. The model parameters are disposed two by two making easy to find the zone where the best solution of adjacent parameters may be possibly situated. The algorithm employed was the Ant Colony Optimization for continuous domains which have not converged and the complete execution has required approximately $32 \mathrm{~K}$ model evaluations as the default options for the metaheuristic are 500 iterations using a population of 64 ants. The previous value is certainly not acceptable for a costly individual based model which may require from several hours to days for such high number of evaluations. Thus, it is necessary to tune the metaheuristics for reducing the total 
number of iterations or alternatively using the algorithm introduced in this work ees 2 for making the initial tour to the solution landscape. The ees 2 is not intended to find the minima but instead it is well suited for partitioning the problem space towards the good solution zones. The 5.10 shows the contour plots generated using ees 2 which required just 600 model evaluations for reducing the solution zone. The global minimum for the Rosenbrock function of $N$ variables is zero and is found setting all variables to 1 .

The Figures 5.9 and 5.10 are demarcating the possible zones were the problem solution can be found. In the case of Rosenbrock test function, which has been used in these examples, the best solution is known a priori to be zero when all parameters are 1. Thus, looking on the first plot, it can be easily observed that the hot zones marked with best fitness are those corresponding the best problem solution. Nonetheless, the complete solution landscape encompasses a very wide zone when compared to the solution estimated using the ees 2 which shows a much more restricted portion of solution space. It is important to note that real models normally do not generate results so evident as those generated with test functions. Usually, the real individual-based models will show discontinuities and possibly more than one zone with good fitness values owing to the nonlinear or second order interaction between model parameters.

\subsubsection{Comparing metaheuristics}

The task of choosing the most suitable metaheuristic for the parameter estimation problem is not an easy one, mainly because the available algorithms behave differently depending on the problem type. It is also a consequence of intrinsic stochasticity present on individual-based models as well as the random nature of algorithms itself. Therefore, it is hard to provide general recipes for deciding what method is right for a particular problem instance. The algorithms enclosed in this work are tuned with those parameters adequate for general cases but some tweaking may be required for achieving the best results. Specifically, the optimization metaheuristics can be very sensitive to the neighborhood structure and to the parameters controlling the balance between local search which accelerate the convergence speed and breadth of search which may avoid to get stuck in local optima failing to converge to the best solution. It may be necessary some trial and error approach, testing different algorithms, observing the convergence speed, the value of cost function and then tuning the algorithm 
parameters accordingly.

The application of optimization metaheuristics to individual-based models, as mentioned previously, poses an additional problem because every model execution is computationally costly when compared to other models types and, because of that the cost of the algorithm itself can be neglected. Therefore, one of the most important factors for selecting an algorithm is the minimal number of evaluations of objective functions which are needed for finding an acceptable solution satisfying the optimization target. Bearing this in mind, this section provides a systematic comparison between some of the metaheuristics mentioned in this work. The metaheuristics have been compared using the functions known as Cigar, Schaffer, Griewank and Bohachevsky [QLW $\left.{ }^{+} 16\right]$ [JY13] which are standard test functions commonly employed for benchmarking the optimization algorithms. The benchmarks were performed using the four variables version of test functions and the experiments were replicated seven times using randomly selected initial random seeds². The summarized numerical results obtained from the benchmark process are shown on Table 5.2 .

\footnotetext{
${ }^{2}$ The details and the code used for the benchmark are enclosed along the package sources which are available on https://github.com/antonio-pgarcia/evoper
} 
Table 5.2: The output of benchmarking metaheuristics algorithms. These results were produced using the function compare.algorithms1 included in package distribution and are the average values of seven replications with different initial random seeds. The convergence values are the ratio of replications which actually converged over the total number of performed experiments.

\begin{tabular}{llrrr}
\hline Function & Algorithm & Evaluations & Convergence & Fitness \\
\hline Cigar & saa & 458.14 & 1.0 & 0.04702100 \\
& pso & 2621.71 & 1.0 & 0.05033806 \\
Schaffer & acor & 2651.43 & 1.0 & 0.07268033 \\
& ees1 & 332.86 & 1.0 & 0.06308869 \\
& saa & 649.57 & 1.0 & 0.07438586 \\
Griewank & pso & 6269.71 & 0.3 & 0.57479139 \\
& acor & 2249.14 & 1.0 & 0.08662474 \\
& ees1 & 495.71 & 1.0 & 0.08821531 \\
& saa & 501.00 & 1.0 & 0.04579390 \\
& pso & 4214.86 & 0.6 & 0.09860997 \\
Bohachevsky & acor & 3812.57 & 1.0 & 0.07698225 \\
& ees1 & 308.57 & 0.9 & 0.07089164 \\
& saa & 258.14 & 1.0 & 0.05232314 \\
& pso & 5053.71 & 0.4 & 0.44120018 \\
& acor & 1362.29 & 1.0 & 0.04323902 \\
& ees1 & 258.57 & 1.0 & 0.06660127 \\
\hline
\end{tabular}

The experiments were conducted setting a tolerance level of $10^{-1}$ in order to avoid a time-consuming process and for mimicking tolerance levels which may be relevant for individual-based models which may be considered to converge with higher values than plain mathematical functions. These values can be taken as a starting point for deciding what algorithm is most likely to provide acceptable results for the optima with lower number of model evaluations. The Figure 5.11 presents graphically the results for the benchmark, showing the total number of model evaluations required for model converging with the provided tolerance level. As can be observed, the evolutionary strategy 1 (ees1) metaheuristic consistently require fewer evaluations of objective function than the other algorithms, excepting for the Bohachvsky function which required practically 
the same number of evaluations as the second better algorithm which is the simulated annealing (saa). The third best algorithm is the ant colony for continuous domains (acor) followed by particle swarm optimization (pso) that curiously has not behaved as expected with the parameters tuned according to the recommended values [Cle12] and we are evaluating other combination of parameters and neighborhood functions.

The Figure 5.12 shows the value of objective function when the algorithm terminates wither when convergence criteria is met or when the algorithm reaches the maximum number of configured iterations. It is important to note that the comparisons shown here are just an initial set of hints for providing a general overview for behavior of each of the algorithms mentioned in this work. The fact that simulated annealing algorithm has been the best performer is the expected result because, differently from other algorithms, it is using a population of size $N=1$ which means that for each iteration only one individual problem solution is being evaluated. The other algorithms, by default are using values of $N=16, N=64$ and $N=10$ respectively the number of particles of particle swarm optimization, the number of ants of ant colony optimization and the solution size of evoper evolutionary strategy 1 . This is one of the factors causing the differentiate performance figures.

Consequently, the initial parameter set, defined of each algorithm, should be seen as the starting point for tuning the metaheuristics for achieving the desired results and a considerable amount of testing may be necessary for getting the best results for the parameter estimation process of a particular model instance. Additionally, some algorithm can be more adequate for a problem than another, therefore, checking the initial outputs of multiple algorithms limiting the number of iterations, may be an interesting exercise for choosing the most suitable metaheuristic.

\subsubsection{Parameter estimation of individual-based models}

One of the remarkable aspects is that the syntax is simple and consistent independent of the function for which parameters are being estimated which means that the set of API primitives required for applying the metaheuristics are the same independently if the target model is a plain mathematical function, an ODE, an individual-based model implemented in Repast or in any other environment for with an add-on have been implemented. The following example consists in the search for the best parameter com- 
bination which minimizes the discrepancies between the simulated values for bacterial conjugation produced using the BactoSIM simulation model [PRP15b] [PRP15a] and the experimental values for conjugative plasmids taken experimentally [dCRC ${ }^{+} 12$ ]. The model provides several outputs but only two values will be used as reference for the parameter estimation process: the conjugation rates and the doubling time for donor and transconjugant cells because experimental observations are available only for these variables. Both model outputs conjugation rates and the doubling time are time series but we will just compare the first for every simulated time step and for the generation time the overall average will be employed for defining the cost metric. The conjugation rate metric used in the simulation experiments is the ration between the number of transconjugant cells and sum of transconjugant cells plus the uninfected recipients, defined as $T /(T+R)$. For building the metrics for comparing both experimental and simulated time, two approaches have been explored, one using a simple metamodeling [JDC03] [Sal08] using a linear model fit to the model output and the observed data and another using the dynamic time warping technique [ $\left.\mathrm{LFLZ}^{+} 15\right]$. These alternative approaches are presented In Figure 6.7 where the functions my.cost1 and my.cost 2 shows respectively the implementations of cost function using a metamodel and the dynamic time warp algorithm[Gio09] for comparing experimental and simulated conjugation rates time series. This example shows how to find the best combination of model parameters which minimize the differences between the observed and the simulated data for the simulated variables conjugation rate and doubling time.

The implementation of cost function shown in Step 1a creates two simple linear regression models of observed and simulated data for comparing the slope and the intercept coefficients which are serves as the distance metric for measuring how close are both time series using root mean square deviations. The cost function also considers the values of doubling time for creating a composite metric. Additionally, the function my.cost1 uses a hybrid categorical-quantitative metric for the doubling time output which is described in the Equations (5.3) and (5.4), respectively the cost estimator for the doubling time of donors (D) and transconjugant (D) bacterial cells. Basically, the cost is zero if the values estimated by the model falls within a limited range around the average value of experimental data or the root mean square devia- 
tion (RMSD) between the simulated and observed values otherwise.

$$
\begin{aligned}
& \mathcal{C}\left(g_{D}\right)= \begin{cases}0, & \text { if } 42 \leq g_{D} \leq 62 \\
\operatorname{RMSD}\left(g_{D}, 52\right), & \text { otherwise. }\end{cases} \\
& \mathcal{C}\left(g_{T}\right)= \begin{cases}0, & \text { if } 33 \leq g_{T} \leq 53 \\
\operatorname{RMSD}\left(g_{T}, 43\right), & \text { otherwise. }\end{cases}
\end{aligned}
$$

In both cost functions my.cost 1 and my.cost 2 , the first 60 minutes are eliminated from being used in the distance metric because during that time lapse the experimental data is a somewhat noise, possibly due to the lagging time or the adaptation to the culture medium. It is also worth to mention that before undertaking a full length run, which certainly would last a large amount of time, would be much better start trying several algorithms with a limited number iterations for getting an initial map of problem solution or using the ees 2 metaheuristic which is limited to 600 evaluations of objective function. An example of an initial mapping for a real individual-based model is shown in Figure 5.14. One of the things that can be perceived at a first glance is that this plot have more diffuse borders delimiting the best zones of problem solution space than the examples presented previously in Figures 5.9 and 5.10 where the object of study were plain mathematical functions. That is the commonly observed pattern for real models owing to the stochasticity and the nonlinear iterations between the model elements. Hence, it is normally necessary to make several initial mappings of the problem for recognizing the zones of best fitness and then making the adjustments on the initial parameter ranges for achieving best estimation results.

Returning to the Figure 5.14, despite of the lack of clearly defined limits for best parameter combinations, it can be observed that some zones are generating better cost values than others. Of course, the process should not be guided by just one algorithm with a single run but a more exhaustive exploration with several runs of available algorithms, also using different sets of random seeds. But for the sake of brevity and just illustrating the process, we will extract conclusions from this single execution. Thus, the first contour plot which shows the parameters cyclePoint ${ }^{3}$ and conjugationCost

\footnotetext{
${ }^{3}$ The cycle point parameter represents the point of time, from cell birth to division when the conjugation is most likely to happen.
} 
allows to detect interesting zones for both parameters circumscribed to those values between $25 \%$ and $75 \%$ with peaks for cyclePoint settled approximately over values of $40 \%$ and $70 \%$ and the conjugationCost being close to the $50 \%$. The plot relating the conjugationCost and the piliExpressionCost shows similar results for the first parameter as in the previous case, which have its better fitness values nearby the $50 \%$ of cell cycle, moreover the second has three promising zones at $25 \%, 40 \%$ and $70 \%$. Finally, the last two plots, relating the piliExpressionCost-gammaO and gamma0-cyclePoint are far from being conclusive but seems to indicate better performances rounding the zone of $\gamma_{0}=5$. The next step in the analysis of parameter space may include refining somehow the initial assumptions about the parameter bounds, for instance, limiting the initial range of parameter cyclePoint for the values found to contain better cost (25\%$75 \%$ ) and running again the exploratory analysis. Therefore, adjusting the range and running the simulated annealing algorithm with the maximum number of iterations limited to 50, new results are obtained and presented on Figure 5.15. These exploratory steps can be repeated a number of times before staring a complete run of metaheuristics. 

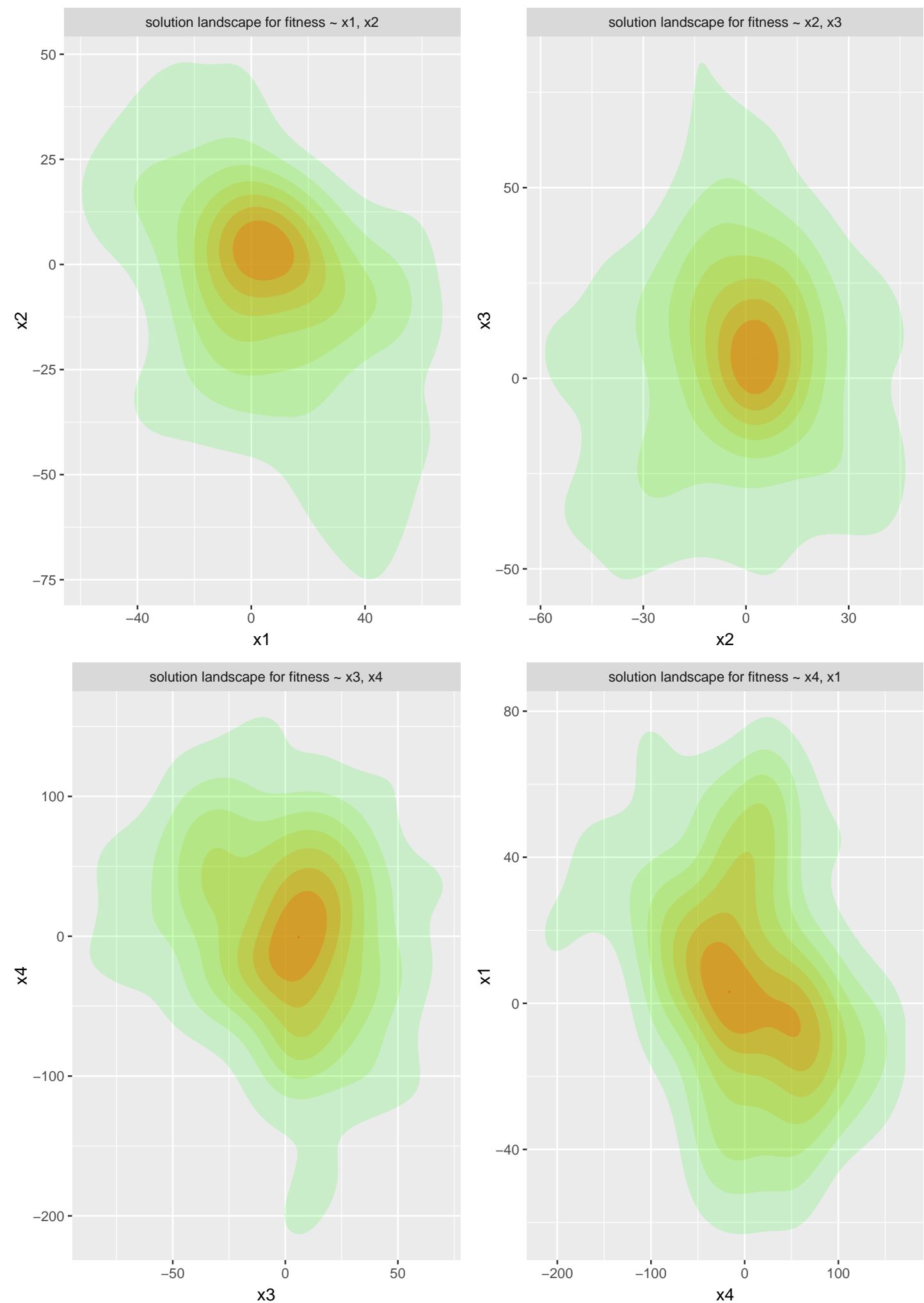

Figure 5.9: Exploring solution landscape for visited space generated during the execution of Ant Colony for Continuous domains (acor) algorithm. The four contour plots provides a panoramic view for the fitness surface of the variable pairs $(\mathrm{x} 1, \mathrm{x} 2),(\mathrm{x} 2, \mathrm{x} 3),(\mathrm{x} 3, \mathrm{x} 4)$ and $(\mathrm{x} 4, \mathrm{x} 1)$. The contour curves are employing a color scheme, from light green to red for indicating the cost value, which means respectively the worst and the best fitness for the function. 

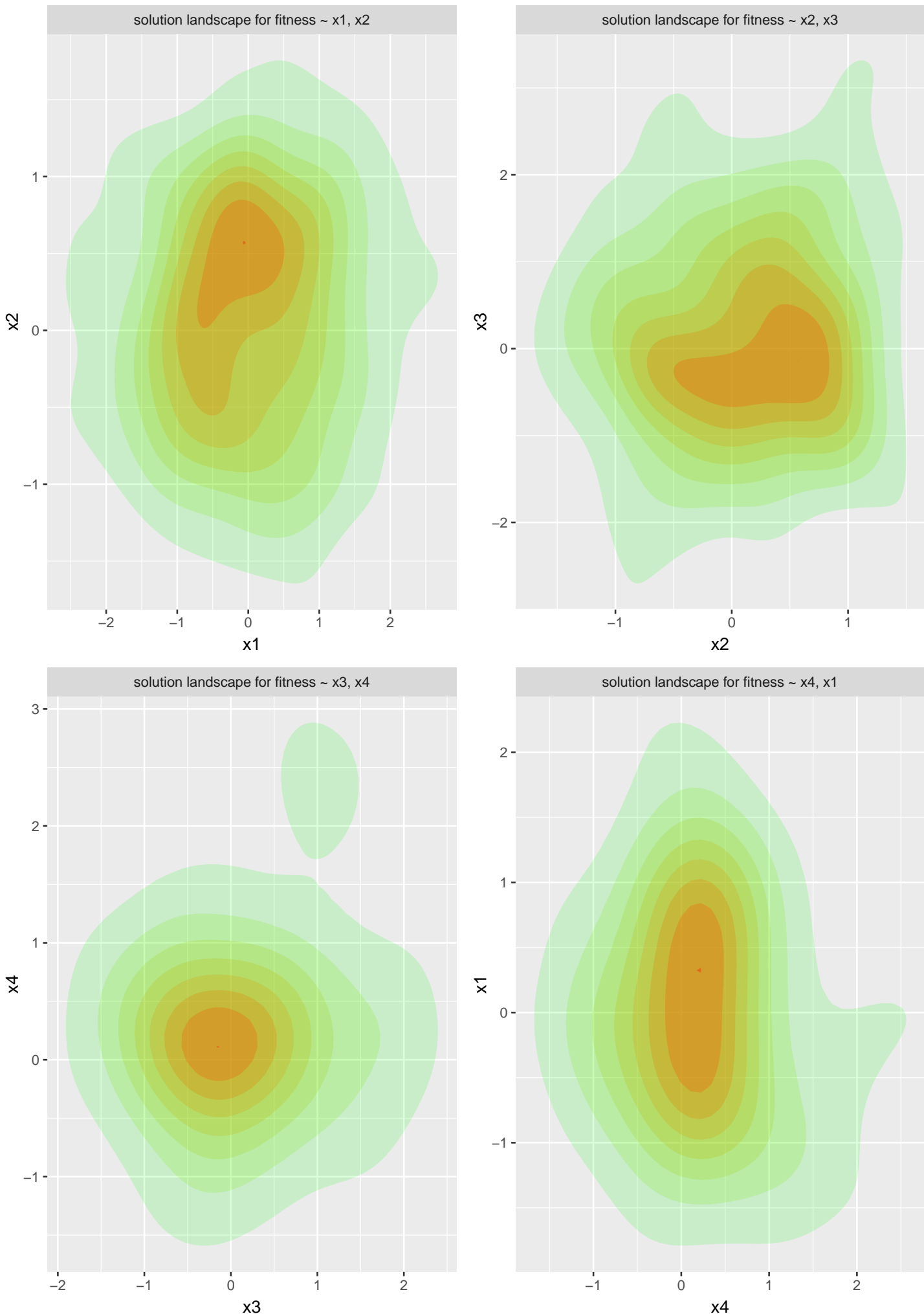

Figure 5.10: Exploring solution landscape for visited space generated during the execution of EvoPER Evolutionary strategy 2 (ees2) algorithm. The four contour plots provides a panoramic view for the fitness surface of the variable pairs $(x 1, x 2)$, $(\mathrm{x} 2, \mathrm{x} 3),(\mathrm{x} 3, \mathrm{x} 4)$ and $(\mathrm{x} 4, \mathrm{x} 1)$. The contour curves are employing a color scheme, from light green to red for indicating the cost value, which means respectively the worst and the best fitness for the function. 

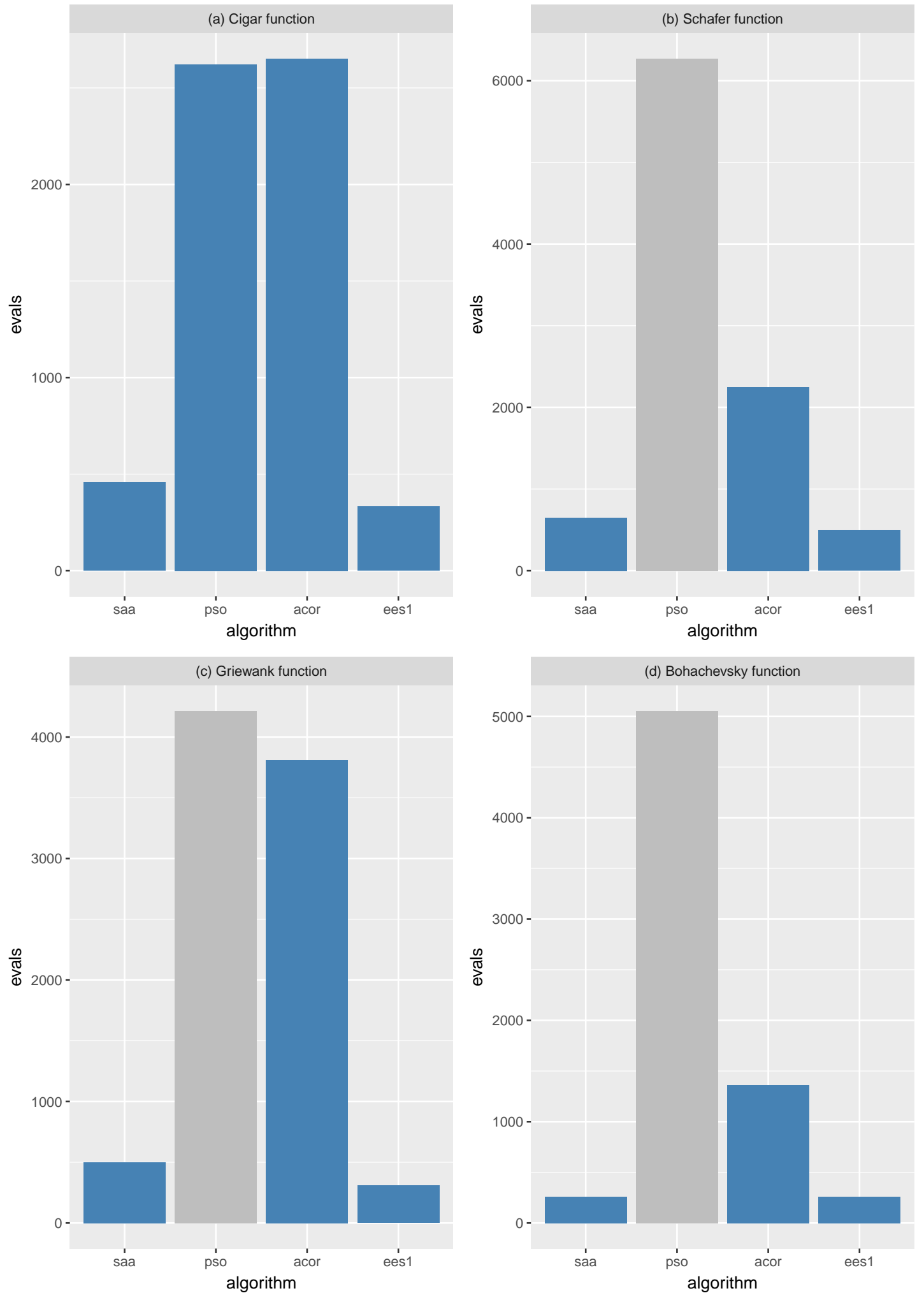

Figure 5.11: Comparing the number of objective function evaluations required for each algorithm. The subplots show the average number of model evaluations which the metaheuristics required for reaching convergence using the benchmark functions (a) Cigar, (b) Schaffer, (c) Griewank and (d) Bohachevsky. The meaning of the gray bar is that number of experiments which algorithm converge were inferior to $60 \%$. 

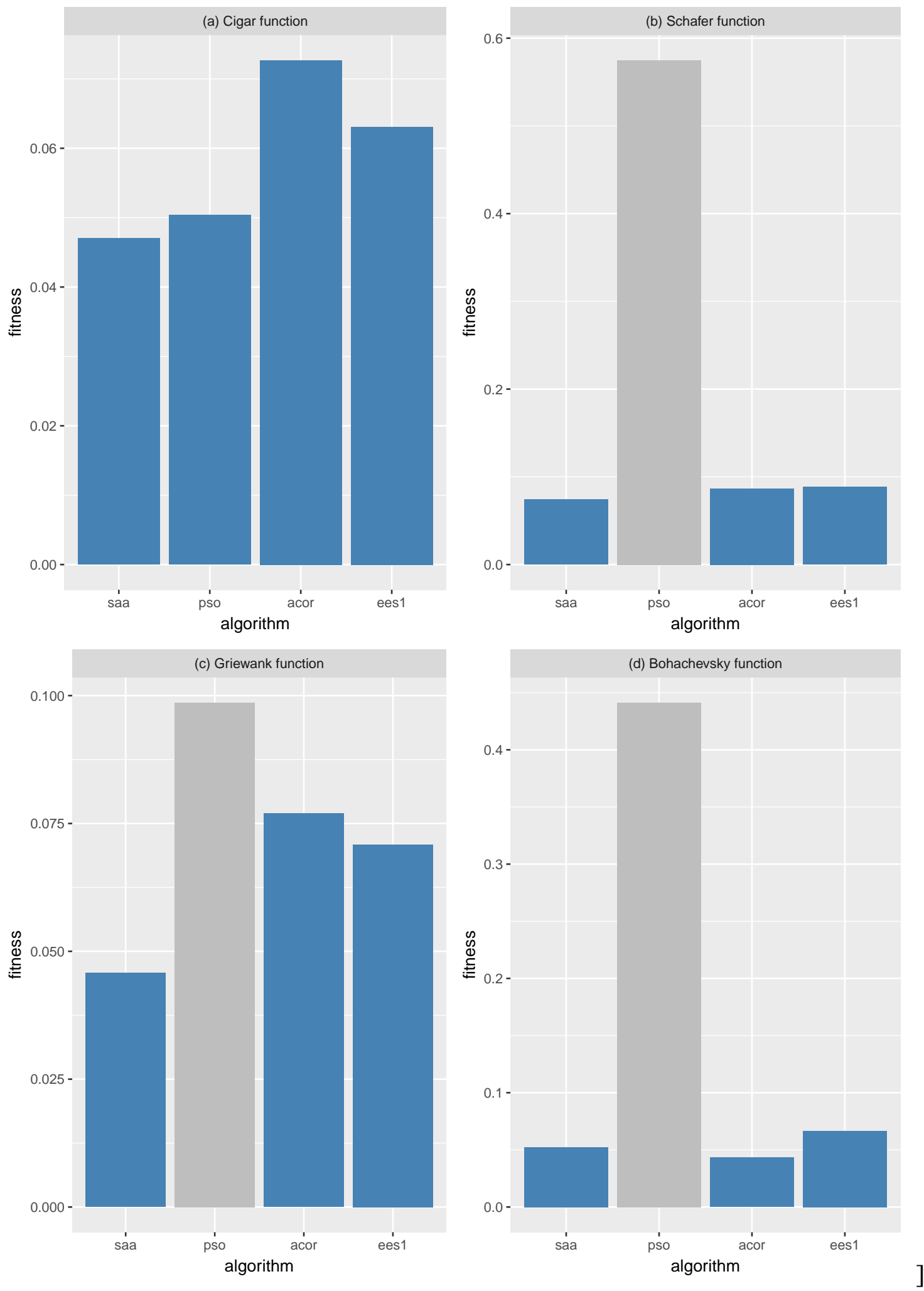

Figure 5.12: Comparing the final value of objective function (fitness) for each algorithm. The subplots show the average fitness value for the benchmark functions (a) Cigar, (b) Schaffer, (c) Griewank and (d) Bohachevsky. The meaning of the gray bar is that number of experiments which algorithm converge were inferior to $60 \%$. 


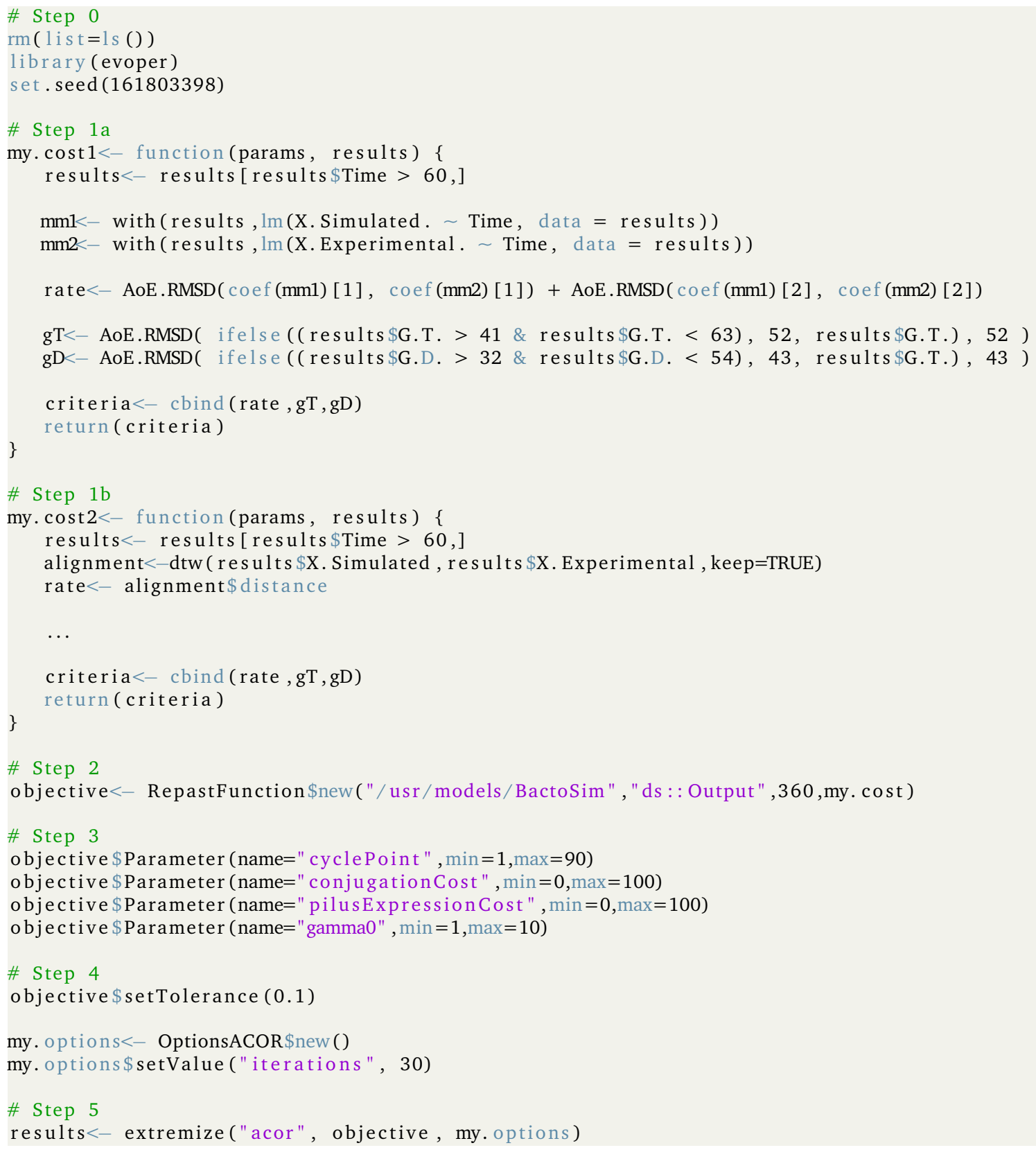

Figure 5.13: The code required running the parameter estimation for an individual-based model using the Ant Colony Optimization algorithm. This code snippet shows the implementation details for two alternative implementations of cost function. As can be seen, the sequence of steps required are: Step 0 loading the required libraries and sets the random seed; The Step $1 a$ and Step $1 b$ are the implementation of two alternative cost functions one using a metamodel fitted to the simulated data and another the dynamic time warping distance as the cost metric; Step 2 Creates an instance of a RepastFunction class for the underlying model, initializing the model directory and the total simulated time; The Step 3 initialize the model parameters of interest which can be a subset of all declared parameters; The Step 4 shows the creation of a non-default options class for setting the maximum number of algorithm iterations to 30; Finally, in the Step 5 the extremize function perform the optimization of cost function. 

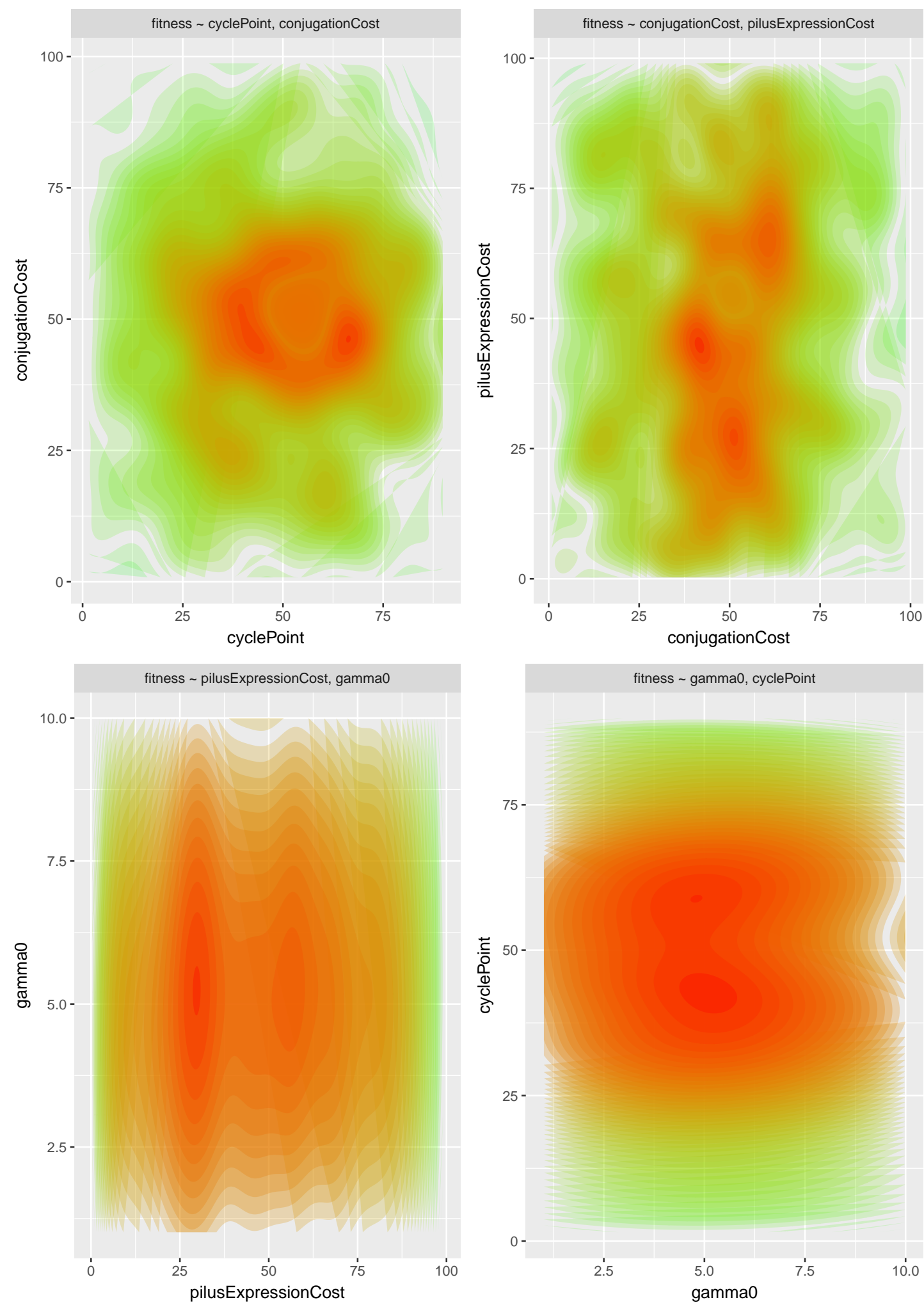

Figure 5.14: The mapping of solution space of BactoSIM individual-based model of bacterial conjugation dynamics. The series of four contour plot shows the effects on the fitness value, defined by the cost function $m y$.cost 1 , for the different values of parameter explored by the optimization algorithm. 

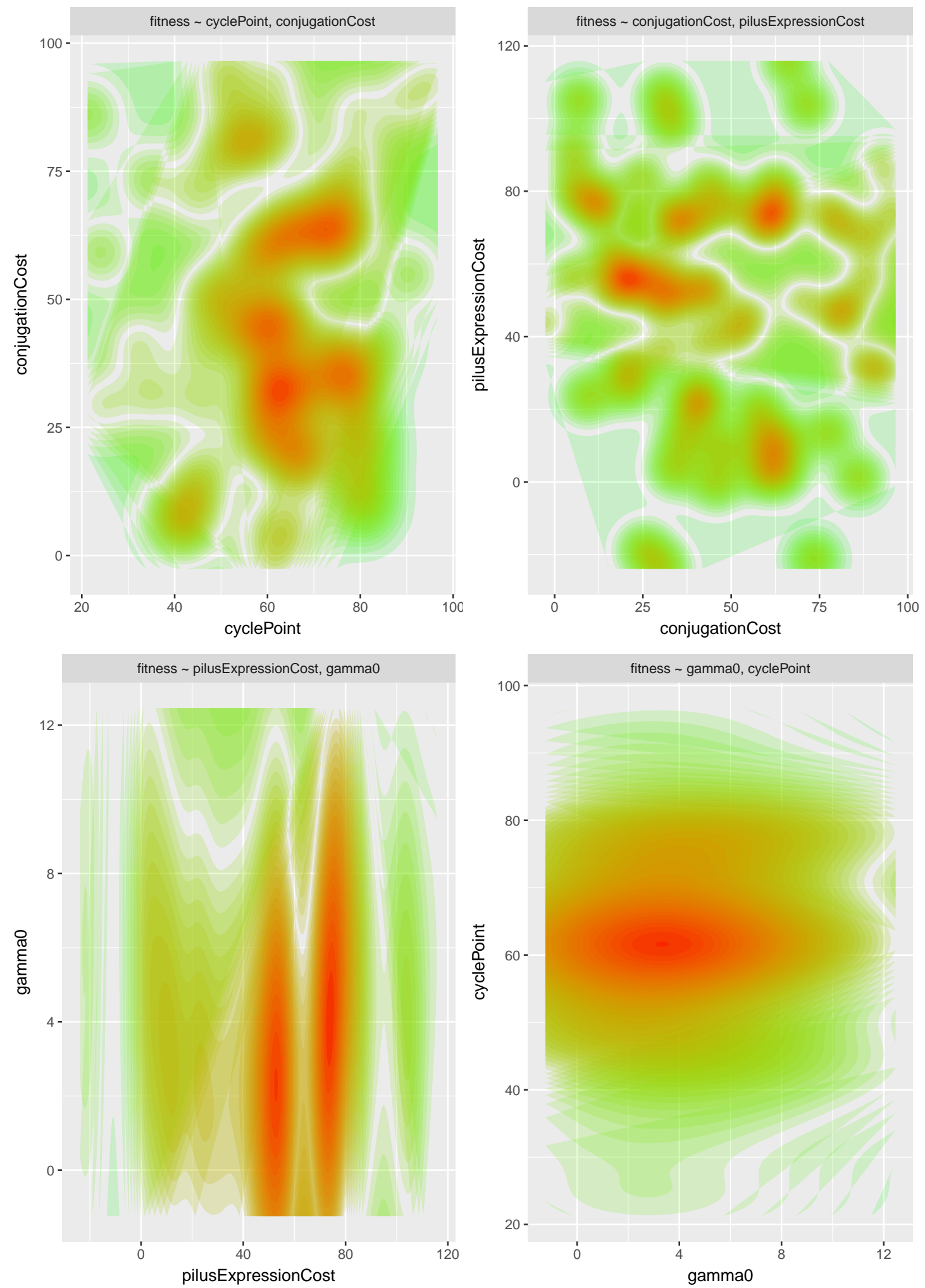

Figure 5.15: Refining the initial mapping of solution space of BactoSIM limiting the variation range of cyclePoint parameter based on the previous mapping results. 


\subsection{Summary}

The systematic parameter estimation should be a fundamental part of individual-based modeling but it is normally omitted by modelers. One of the main reasons is the relative complexity of available methods, the effort required for applying them and the lack of simple tools for the practitioners which usually come from different domains with different backgrounds. Ecology has always greatly benefited from the application of mathematical models to the description of complex processes and iterations. Recently, Individual-based models are becoming a lingua franca for ecological modeling but normally the acceptance of results produced are hindered by lack of a thorough parameter estimation and analysis. The cause may be attributed to the deficit of experience with methods and techniques required for carrying out the analysis of simulation output. The individual-based models are complex, stochastic and non-linear in their nature, therefore the evaluation input parameters for making the model reproducing reliably the reference data is a hard computation task. The best available approach is to estate the parameter estimation as an instance of an optimization problem and apply the existing arsenal of optimization metaheuristics.

Beating this in mind, we have introduced in this work the partial set of features available on EvoPER package alongside with illustrative usage cases, including one application to a real individual-based model with the interpretation of outputs produced and the steps necessary to the complete parameter estimation of the model. The package is being developed keeping in mind the idea of minimizing the effort required to the application of sophisticated methods in the parameter estimation process of Individual-based models. This package allows the modelers to try different alternatives without having to code ad hoc and complex integration code to the existent packages. 


\section{Chapter

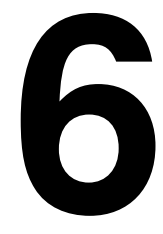 \\ Sensitivity analysis of individual-based models}

6.1 Introduction . . . . . . . . . . . . . . . . . . . . . . 111

6.1 .1 Model development . . . . . . . . . . . . . . . . . 114

6.1 .2 Sensitivity analysis . . . . . . . . . . . . . . . . . . 116

6.2 Overview of R/Repast package . . . . . . . . . . . . . . . . 123

6.2 .1 Design . . . . . . . . . . . . . . . . . . . . . . . . . 124

6.2 .2 The R/Repast R API . . . . . . . . . . . . . . . . . 126

6.2 .3 The objective function interface . . . . . . . . . . . . . . 131

6.3 Examples overview . . . . . . . . . . . . . . . . . . . . 132

6.4 Example 1: BactosIM . . . . . . . . . . . . . . . . . 134

6.4 .1 Model analysis . . . . . . . . . . . . . . . . . . . 135

6.5 Example 2: Predator-Prey . . . . . . . . . . . . . . . . . . 136

6.5 .1 Model description . . . . . . . . . . . . . . 136

6.5 .2 Entities, State variables and scales . . . . . . . . . . 137 
6.5 .3 Model analysis . . . . . . . . . . . . . . . . . . . . . 139

6.6 Example 3: T4SS Common Pool . . . . . . . . . . . . . . . 142

6.6 .1 Model description . . . . . . . . . . . . . . . . 142

6.6 .2 Process overview and scheduling . . . . . . . . . . 143

6.6 .3 Analysis of model $\ldots \ldots \ldots \ldots$. . . . . . . . . 145

6.7 Summary . . . . . . . . . . . . . . . . . . . . . . . . . . 147 


\subsection{Introduction}

Computational ecology is a relatively young field which relies extensively on mathematical computational methods and models for studying ecological and evolutionary processes. It is based on the construction of predictive and explanatory models as well as the quantitative description and analysis of ecological data [ $\left.\mathrm{HCD}^{+} 95\right]\left[\mathrm{PPH}^{+} 12\right]$. The continuous growth of computational power available for and end users, the existence of tools and the constant increment of empirical data available, makes viable for many scientists to develop and simulate tremendously complex models from their desktops. In addition, the intrinsic characteristics of ecological processes, maxim their temporal and spatial scale [DLM00], converts the task of carrying out controlled experiments a physical impossibility. Hence, in most cases the only feasible alternative is to simulate the process in order to make experiments spanning the full length of ecological and evolutionary scales. The computational ecology has its roots from the successful results achieved from mathematical ecology which has proven to be an essential tool for understanding the complexities which arise from ecological interactions.

It is a widely accepted that simple models with a small number of state variables and parameters provide best generalizations than the complex ones [Smi74] [EGJ ${ }^{+13}$ ] with a clear distinction between simulation models and theories as separate entities handling different kind of problems. It has been recently questioned the correctness of the idea the simple models lead to generality in ecology [EGJ $\left.{ }^{+} 13\right]$. We believe that the parsimony principle must always be taken into account when developing models, but this has a different meaning depending on the modeling formalism we are using. Simplicity does not have the same meaning when the referred modeling formalism is a deterministic ODE or when it is applied to Agent-based modeling, as long as every modeling techniques has its own idiosyncrasy and constraints. The Agent-based modeling is a flexible and versatile abstraction where the whole system under study is described or formalized by its component units, which facilitates a more natural description of a system and the comprehension of individual properties leading to the emergent phenomena [Bon02].

The Agent-based models (AbM) are much more fine-grained than their wholepopulation aggregated counterpart and as consequence they tend to be more complex requiring more equations, parameters and processes in order to represent the same 
phenomenon. That is not intrinsically a problem or a quality but simply a constraint imposed by the modeling formalism in use and it is up to the modelers to find the correct tradeoff between the purpose of the model and the level of details which should be part of the model structure.

The AbM have been being established progressively as a main-stream and valuable tool for modeling complex adaptive systems in many distinct areas of knowledge, ranging from social science, economics to any flavor of computational and systems science such as biology, ecology and so on [GR05b]. The reason is, amongst other things, the relative ease with which detailed structural information can be incorporated into a model without the constraints of other methodologies [HB09]. Nonetheless, the possibility of incorporating many details comes with the cost of models with a high complexity level, containing many rules and parameters for which the exact values are, in many cases, hard or impossible to determine experimentally, that is what is known as parameter uncertainty. When used in the context of ecological systems the Agent-based modeling is also known as Individual-based modeling (IbM) [GR05b].

The distinctive aspect defining what is an IbM is that individuals are represented by discrete entities and they also have a property or state variable which are unique in the population being simulated [Ber02]. Hence IbM is a valuable abstraction for simulating populations, communities or ecosystems capturing the individual variability, randomness and their complex dynamics. It is a bottom-up approach where the system under study is modeled using mechanistic explanations on the interacting system parts [FPL08]. Therefore, the global behavior shown by the system as a whole, is an emergent property derived from the local rules defining the individuals. That is particularly useful testing different hypothesis or phenomenological explanations for the individual processes in order to verify which of them are producing the global observed behavior [Pas05]. Moreover, differently from aggregate models, it is customary that IBM have a large number of state variables and parameters which in most cases are hard or directly impossible to elucidate experimentally leading to many levels of uncertainty in this kind of models. In order to tackle with the uncertainty and for making robust predictions, we have to use a sound methodology for applying what-if analysis to check how stable are the model outputs when varying the input parameters [TKG14]. There exist a large set of mathematical tools for analyzing the model output which are known generically as sensitivity analysis. Normally applying these techni- 
ques are cumbersome, requiring a lot of effort from modelers, hindering the throughout analysis of computational models.

According to [TKG14] the most of Individual-based models published tends to omit the systematic analysis of model output, mainly because modelers normally do not have the specific knowledge to implement the required methods. Therefore, it seems clear, that the availability of simple and user friendly tools for experiment design and analysis would greatly help modelers to improve the formal quality of their models.

In other scientific fields, which are strongly rooted on an extensive experimentalism, is practically impossible to conduct any kind of research without a well-designed experimental setup and a further statistical analysis and hypothesis test. Perhaps the reasons are that these experimental fields already have a complete and mature toolbox for design and evaluation of experiments [LH78] [MW95] leaving no room for deviation from these standards. On the other hand, in silico based experiments are still on early stage and verification and validation procedures are not well established yet. In addition, the real value of a computational model depends much on the ability of other researchers to reproduce and enhance the results elsewhere; in other words, results must be reproducible. Hence, in order to achieve reproducibility, research methods should be stated clearly and should preferentially being backed by standard methods and software tools.

Bearing this in mind we introduce R/Repast a GNU R package for running Repast [NCO $\left.{ }^{+} 13 \mathrm{~b}\right]$ models from GNU R environment as well as for carrying out global sensitivity analysis on the model results. In the following sections we will contextualize the problem providing a basic background for understanding what is being addressed in this work and we will also provide a basic description about the package functionalities. Finally, we will show three worked examples on how the package can help modelers to make the conclusions drawn from model results much more robust. The first example explores the basic aspects of bacterial conjugation process. The second is an individual-based implementation of the classic predator-prey model enclosed as part of the standard Repast Simphony distribution. Finally, the last example was developed ex professo for this work and it is an instance of common pool problem in the context of two plasmids "sharing" the genes required for the expression of conjugative system. 


\section{Background}

\subsubsection{Model development}

Model development is an iterative and objective driven activity and the first step required to develop a model is to have a clear and ideally unambiguous statement about the model purpose. Therefore, every experimental study carried out using modeling and simulation should follow the experimental life cycle based on the successive sequence of four cyclic steps, starting from (a) Conjecture, which defines the model purpose and why the model is being developed; (b) Design phase where the model is translated to some runnable implementation; (c) Experiment step which means the execution of model following a well-established plan oriented to confirm or reject the initial conjecture and finally the (d) Analysis step where the data generated in the previous step is analyzed with a sound methodology which, hopefully will generate new insights, uncover model flaws and iteratively improve the initial conjecture and design [BD87]. A simple graphical representation of these four iterative steps is shown in Figure 6.1.

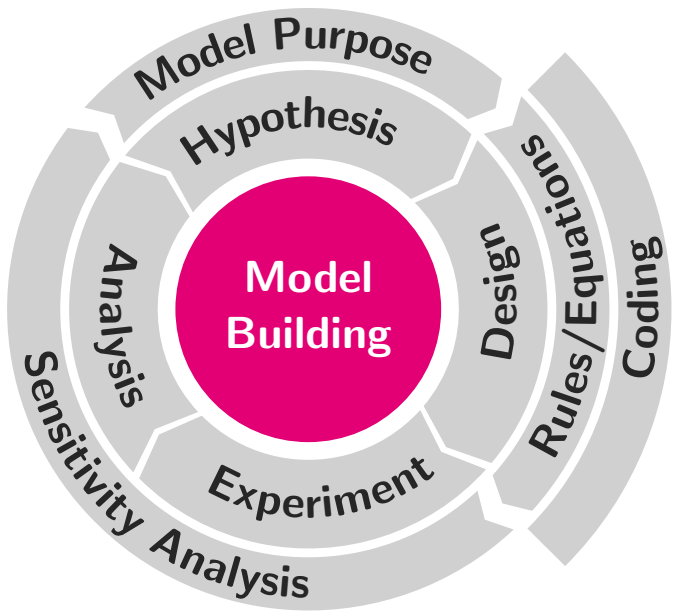

Figure 6.1: The iterative model development life cycle. This figure shows the relationship between the modeling phases and their associated tasks when applied to an individual-based model.

Part of design phase consist in convert the model equations and rules to a computer 
code implementation. Currently there are several frameworks available for developing individual-based models. These frameworks are designed to address some specific requirement such as usability [TW04], flexibility or scalability [NCO $\left.{ }^{+} 13 \mathrm{~b}, \mathrm{LCRP}^{+} 05\right]$ or support to multiple modeling paradigm, such as AnyLogic [ESJ07]. Certainly, the most widespread framework in ecological modeling is NetLogo [TW04] which is considered to provide an easier development environment based on extensions to Logo paradigm especially suited for those which are not much familiar with modern programming languages. One of the main drawbacks of NetLogo is the scalability. NetLogo tends to show some performance issues when simulating a large number of agents. On the other hand, Repast Symphony framework has a steep learning curve but provides a fast and flexible java-based environment with many interesting features for simulating large scale computational ecology models. These features include, amongst others things, the integration with Weka, exporting the model output to $R$ environment, support for running distributed batch simulations and some built-in facilities for parameter sweeping $\left[\mathrm{NCO}^{+} 13 \mathrm{~b}\right]$. Finally, Mason is, in some extent, very similar to Repast but less mature than it is; It has been designed focusing on providing faster execution speeds $\left[\mathrm{LCRP}^{+} 05\right]$. The only of these frameworks providing integrated sensitivity analysis capabilities is AnyLogic, the other frameworks: NetLogo, Repast and Mason which are all free software do not have built in support to sensitivity analysis.

The Repast framework is widely used in many different fields for building individualbased simulation models of dynamic processes [WNF ${ }^{+}$15] [GBP ${ }^{+}$15] [TLNV15]. In addition, Repast also has a framework for high performance computing using the $\mathrm{C}++$ programing language with similar conceptual entities as those found in Repast-java. Repast also has support for running GNU R code [R C15] [Cra07] from inside the user interface but until now it has not been feasible to run Repast models from $R$ environment for controlling model in order to implement experimental designs, calibration, parameter estimation and sensitivity analysis, therefore hindering a throughout and comprehensive validation of Individual-based models developed using Repast Simphony. 


\subsubsection{Sensitivity analysis}

Because of sensitivity analysis is a broad and complex subject, a throughout discussion would be lengthy and out of the scope of this work. Instead we will try to provide a more amenable and practical approach keeping the discussion at a general level but rigorous enough to let the practitioners gain the knowledge required to understand, apply and interpret the results. For a more detailed review please refer to [STCR04] $\left[\mathrm{PBF}^{+} 16\right]$. It is interesting to start the discussion providing the exact meaning of some the many expressions which are used commonly in the analysis of models. There are several terms used in the context of sensitivity analysis for which is important to provide the formal meaning. For instance, the jargon of sensitivity analysis includes model calibration and parameter estimation which many times are used as they were equivalent, even though they are different objectives. Other terms such as uncertainty analysis, omitted variable bias, objective function or cost function are also important part of SA lexicon.

Generally speaking, the objective of $S A$ is to understand the effect of varying input factors on the model output [STCR04]. Under this very general statement we have a wide range of methods and techniques which are suitable for distinct kinds of models. In order to improve this definition, it is convenient to provide a more formal definition to the entity which is the target of SA: the model. Formally speaking, a model is a functional relation between a number $k$ of input factor, also called independent or predictor variable and the output variable, sometimes referred as dependent or response variable [BD87] as depicted by the expression $\eta=f\left(x_{1}, x_{2}, \ldots, x_{k}\right)$, being $\eta$ is the average value of response variable considering any specific setting for the input factors $x_{i}$. Therefore the value of a single model run is given by $y=f\left(x_{1}, x_{2}, \ldots, x_{k}\right)+\epsilon$, where $\epsilon$ is difference between the value of $y$ and the expected value $E(y)=\eta$. The error $\epsilon$ is consequence of stochasticity introduced by design in the structure of model to capture the population variability. Finally, recognizing that most real world models usually have more than one response variable, the structure of an individual-based model $M$ can be generalized for $n$ outputs as can be seen below 


$$
M=\left\{\begin{array}{l}
y_{1}=f_{1}\left(x_{1}, x_{2}, \ldots, x_{k}\right)+\epsilon \\
y_{2}=f_{2}\left(x_{1}, x_{2}, \ldots, x_{k}\right)+\epsilon \\
\vdots \\
y_{n}=f_{n}\left(x_{1}, x_{2}, \ldots, x_{k}\right)+\epsilon
\end{array}\right.
$$

Therefore, being $y_{i}$ some output of model $M$, the model calibration process consists in comparing these outputs to some reference values [ZPK00] which are normally, in the case of ecological or biological studies, experimental or observed data. The target of calibration process is minimizing the discrepancies between simulated and reference values. The function used for computing how far $y_{i}$ output is from the reference values is known as objective function or cost function. There are many options for implementing the objective function and the only requirement is that the return of objective function should be inversely proportional to the quality of fit, being zero the return value for the perfect fit. Common implementations for objective function are based on the definition of acceptable ranges, least squares or even a combination of both. For instance, let $y_{i}$ be the output of some hypothetical model $M$, assuming this variable represents the net reproductive rate $R_{0}$. The reference values $R_{v}$ for the output variable must fall between 0.8 and 1.2 , hence any $y_{i}$ value within this interval is considered to have a perfect fit, bearing this in mind the cost function could be given by the following expression

$$
C\left(y_{i}\right)= \begin{cases}0, & \text { if } 0.8 \leq y_{i} \leq 1.2 \\ 1, & \text { otherwise }\end{cases}
$$

That is what is known as categorical calibration criteria [TKG14]. The main drawback of this approach is that it does not provide any information about how far is the response value from the reference value. A better alternative is to apply some distance function $d\left(y_{i}, R_{y}\right)$ to the output and the reference values, even standalone or in combination with categorical calibration. The most commonly used metric is some of the multiple forms of squared deviation but any distance function can be alternatively employed as long as two properties hold: $d\left(y_{i}, R_{y}\right)=0$ if $x_{i}$ and $R_{y}$ are equal and $d\left(y_{i}, R_{y}\right)>0$ when $x_{i}$ and $R_{y}$ are not equal.

Whilst calibration is a general term, meaning fundamentally the comparison of 
some value to a reference value, the term parameter estimation has a more subtle and specific goal. The parameter estimation is normally considered an inverse problem because the objective is finding the values for the model parameters providing the best adjustment to the reference values. In other words, knowing the expected values for response variable the target is estimating the suitable values for the model parameters. Usually the terminology parameter refers to the constants which are part of models with clear distinction between parameters and independent variables, [BA77], for instance in the growth differential equation shown below

$$
d N / d t=r N
$$

the model parameter would be only the growth rate $r$ and the independent variable the time, but for the purpose of this work we consider indistinctly the model constants and independent variables as being parameters.

The two main objectives of sensitivity analysis are understanding how robust are the model results considering the existing uncertainties and quantifying the effect of input factors on the variance of output [STCR04] [ $\mathrm{PBF}^{+}$16] [Law05]. The intrinsic characteristics of individual-based models which relies on mechanistic descriptions favors the production of models with many sub-processes, state variable and parameters. The design is normally based on incomplete knowledge resulting in several levels of uncertainties in the model parameters, in the model response variables and in the model structure itself. The model structure is also related to the identifiability problem where not all model parameters can be uniquely estimated. The sensitivity analysis can be also used for assess the effect of model structure on the output considering the alternative model implementations as being another parameter. This can be useful for analyzing the omitted variable bias, which basically means that some parameter of model can be over or under-estimated because another important parameter was not included in the model structure. The sensitivity analysis can be carried out letting the parameters varying over the full range of parameter space or restricted to a small region close to the average value, respectively referred as global sensitivity analysis and local sensitivity analysis. Sensitivity analysis can also be performed varying one factor at time (OAT) leaving all others fixed or varying all factors at the same time (AAT). The application of second method is required in order to capture interaction between 
parameters and non-linear effects.

The central point of SA methodology is the estimation of sensitivity indices or coefficients. The sensitivity coefficients allow the quantitative comparison of the contributions from distinct parameters to the model output. In its classical form [BA77] the sensitivity indices are defined as the first derivative with respect to some model parameter $x_{i}$. Considering the general model $y=f(X)$, being $X$ the parameter vector of size $k$, the sensitivity index $S_{i}$ is given by

$$
S_{i}=\frac{\partial Y}{\partial x_{i}}
$$

It is also important to take into account that the partial derivatives can have different units, hence can be necessary to scale them in order to make them comparable. In this approach, input factors are perturbed one-at-time, being that measure of sensitivity suitable for local $\mathrm{SA}\left[\mathrm{PBF}^{+16}\right]$.

Several methods for estimate sensitivity indices which are adequate for global sensitivity analysis are available, such as meta-modeling approach [HKB06], correlation based methods, regression based methods, Fourier Amplitude Sensitivity Test (FAST) [XG11], for a more in depth discussion please refer to [TKG14] [STCR04] [Sal08] $\left[\mathrm{PBF}^{+} 16\right]\left[\mathrm{PlwcfSDV}^{+} 15\right]$. The Figure 6.2 show how are related the different methods for assessing the importance of input factors in simulation models, also including screening techniques [BK96] [AH93]. In this work we will focus on those methods based on the variance decomposition which are suitable for a wide range of situations, including those which are commonly found in individual-based models, such as nonlinear mappings between input factors and outputs variables [ZR06]. In addition to first order effects, the variance decomposition methods, also allows the quantification of second order effects sometimes referred as total order effects. Total order effects indices are useful for the assessment of the interaction between factors which cannot be expressed by a simple lineal superposition. 


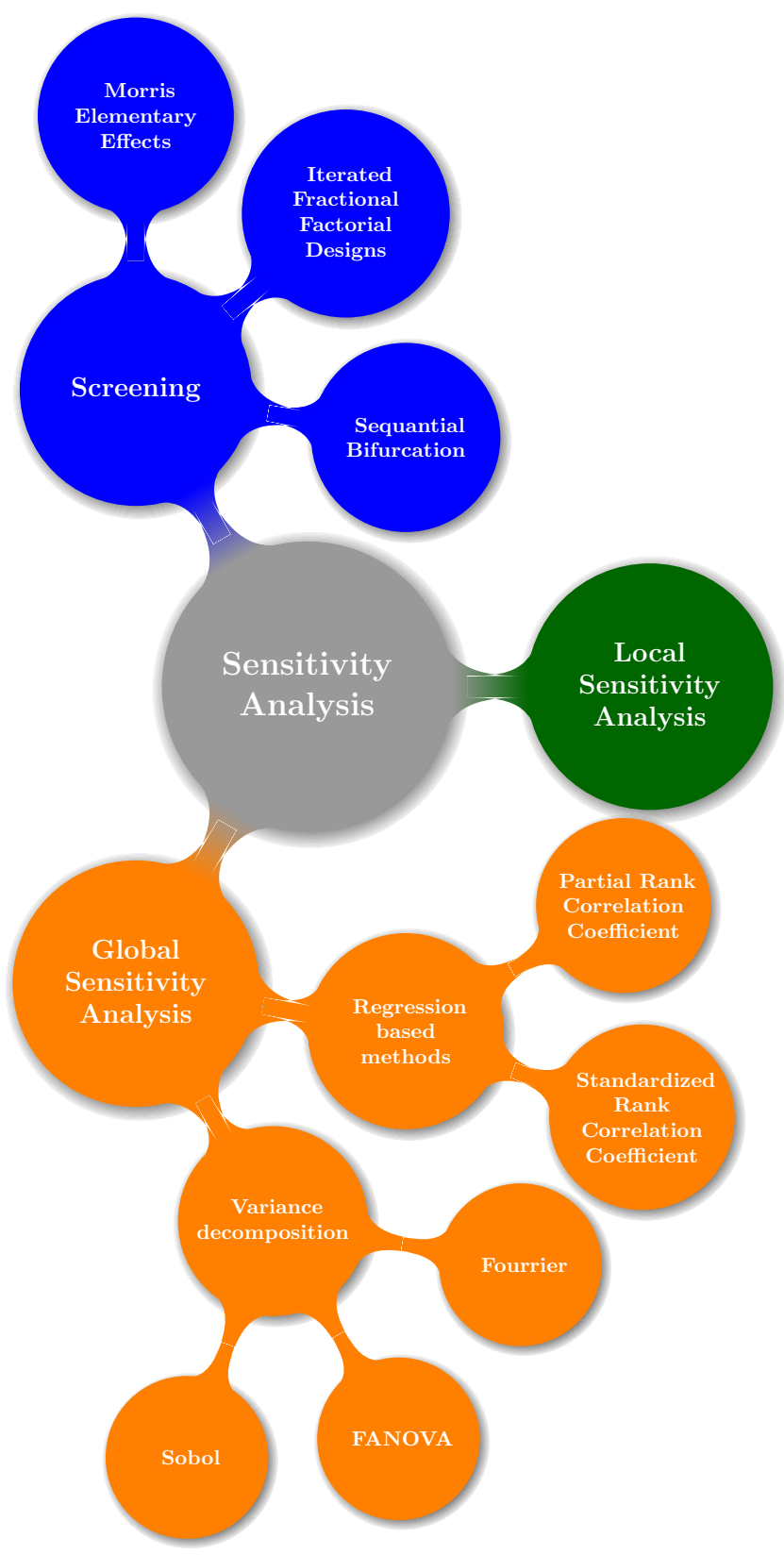

Figure 6.2: The different types of sensitivity analysis and their associated methodologies and techniques.

One of main drawbacks for applying variance decomposition methods on large spatially explicit individual-based models is the requirement of very high number of model evaluations in order to produce consistent results [HKRW13]. An alternative 
approach, in those cases where it is impractical or computationally unfeasible a fully quantitative analysis, is the application of the Morris screening method. The Morris method deliver qualitative information allowing to rank the importance of input factors requiring less model evaluations, which in some case can one order of magnitude inferior to the Sobol method [Sal08].

The Sobol is a method for sensitivity analysis based on the decomposition of the variance of model output and is particularly suitable for discovering the effect of high order interactions between input factors. The interaction means non-linearity where the total effect of two input factors $x_{1}$ and $x_{2}$ on the model output $Y$ are not equivalent to the sum of the individual effects. The general form of sensitivity indices for Sobol methods are shown in Equation (6.1) and Equation (6.2), respectively the first order and total order indices.

$$
\begin{aligned}
S_{i} & =\frac{V_{i}}{V(Y)} \\
S_{T i} & =1-\frac{V(Y)-V_{i}}{V(Y)},
\end{aligned}
$$

where the terms $V_{i}$ and $V(Y)$ are respectively the variance contribution attributed to the ith parameter and the total variance. The expression $V(Y)-V_{i}$ represents the total variance with exception of the variance which is generated by the parameter $i$. The total order index $S_{T i}$ is the contribution of all input parameters but one, the ith parameter, and hence estimating the effect of that parameter on the variance reduction [Sal08].

The total variance $V(Y)$ for a model with $n$ input parameters can be expressed as shown in Equation (6.3) as long as the orthogonality of input factors precondition holds.

$$
V(Y)=\sum_{i} V_{i}+\sum_{i<j} V_{i j}+\sum_{i<j<k} V_{i j k}+\cdots+V_{12 \ldots n},
$$

being $V(Y)$ the total variance from model output and the components $V_{i}, V_{i j}$ and $V_{i j k}$ respectively the variance contribution from the parameter $i$, the variance contribution form input parameters $i$ and $j$ and the variance contribution form input parameters $i$, 
$j$ and $k$. Finally, the component $V_{12 \ldots n}$ express the interactions from all parameters present in the model.

The application of Sobol method, as have been mentioned, can be computationally expensive and sometimes could be useful to reduce the problem dimensionality filtering only the most significant parameters or even simplifying the model structure considering only the parameters accounting for the most of the variability in the model output. It can be accomplished using the Morris screening method to rank the importance of input parameters. The Morris method is an OAT method, meaning that it changes just one factor keeping all other input parameters fixed. The input factors are allowed to vary in discrete levels within the relevant parameter range [Mor91]. The method is considered to be more effective when the number of most significant input parameters are a small subset of model parameters [STCR04].

The original work of Morris [Mor91] define two metrics for ranking input factors which are depicted by $\mu$ and $\sigma$ values ${ }^{1}$. Further, another metric termed $\mu^{*}$ has been suggested by [CCS07] which use absolute values in order to handle effects of distinct signs canceling each other. These metrics for ranking input factors are calculated from what has been termed elementary effects. Therefore, considering a model with $k$ input parameters and being $x=\left(x_{1}, x, 2, \ldots, x_{k}\right.$ any value from the region of experimentation $\Omega$, the elementary effects are calculated according to the Equation (6.4).

$$
e e_{i}(x)=\frac{y\left(x_{1}, \ldots, x_{i-1}, x_{i}+\Delta, x_{i+1}, \ldots, x_{k}\right)-y(x)}{\Delta}
$$

The region of experimentation $\Omega$ is a grid defined by the number of $k$ input factors and by the $p$ discrete levels for every parameter. The recommendations for the values of $p$ and $\Delta$ are respectively that the first should be an even number of levels and the second calculated by the expression $\Delta=p /(2(p-1))$ [Mor91] [STCR04]. The value of $\Delta$ has important implications in the model analysis. It has been shown that in some situations choosing an alternative value calculated as $\Delta=1 /(p-1)$ can detect nonmonotonic behaviors that the suggested standard calculation are not able to capture otherwise [VHBC11].

The metrics of Morris method are calculated over the $F_{i}$ and $G_{i}$ distributions for

\footnotetext{
${ }^{1}$ Not to be confused with population mean and standard deviation
} 
every input parameter. These distributions are generated taking random samples of $x$ from $\Omega$ for calculating the elementary effects and the only difference between them is that $G_{i}$ uses the absolute values of elementary effects $\left|e e_{i}(x)\right|$ as described in [CCS07] [Sal08]. The estimation of Morris metrics are carried out by taking $r$ samples from $F_{i}$ and $G_{i}$ distributions according to the Equations (6.5), (6.6) and (6.7).

$$
\begin{aligned}
\mu & =\sum_{i=1}^{r} \frac{e e_{i}(x)}{r} \\
\mu^{*} & =\sum_{i=1}^{r} \frac{\left|e e_{i}(x)\right|}{r} \\
\sigma & =\sqrt{\sum_{i=1}^{r} \frac{\left(e e_{i}(x)-\mu\right)^{2}}{r}}
\end{aligned}
$$

These three metrics can be used to extract valuable information about the model behavior, in addition to ranking the input factors. For instance, a low value of $\mu$ and a high value of $\mu^{*}$ is high, points that the input factor under scrutiny, possibly has a nonlinear behavior having different signs in function of the system trajectory [STCR04]. A high value of $\mu$ indicates that the input has a monotonic effect on the model output.

The sensitivity analysis methods require significant samples from input space in order to provide reliable results. It is customary to choose between some experimental design [Hic93] for generating the collection of input parameters needed by evaluating the model and allocating the variance contribution of every model parameter. The most generally applied sampling schemas are based on random sampling, full factorial designs or Latin hypercube sampling.

\subsection{Overview of R/Repast package}

In the previous sections we have seen some fundamental ideas on model building and the role occupied by sensitivity analysis methods in the iterative modeling life-cycle. We have also introduced the basic principles of sensitivity analysis focusing on two main techniques namely the Morris Elementary Effects Screening [Mor91] and the Sobol GSA method for variance decomposition [Sal08]. Both methods have a wide 
range of applicability, making them suitable for their use in the analysis of Individualbased models. These methods require the model be evaluated many times with a different set of input parameters, making completely impractical undertaking a manual analysis introducing individual parameters manually on a graphical user interface. The Repast is an extremely flexible framework for object-oriented development of Agentbased models using Java language but it lacks from model analysis tools. On the other hand, the GNU R is a superb open source tool for data analysis with a vast and active community developing and adding new methods to the core R system. Bearing this in mind, we introduce our package R/Repast which bring together the best of both worlds. Roughly speaking, the R/Repast package have two main objectives: (a) Provide an interface for running Repast models from $\mathrm{R}$ and gathering the simulation data generated and (b) Automating the application of sensitivity analysis and simple model calibration methods to the Repast models. The R/Repast is an open source project delivered under the MIT license system. The package provides a powerful and simple $\mathrm{R} \mathrm{AP{ } ^ { 2 }}$ which reduces the code required for running the most commonly used experimental methods suitable for. The software and the user manual can be downloaded from CRAN website and the complete project source code from GitHub repository. Both are available respectively from the following URLs:

- https://cran.r-project.org/web/packages/rrepast/

- https://github.com/antonio-pgarcia/RRepast

\subsubsection{Design}

The R/Repast was intended primarily for invoking Repast Simphony models from inside GNU R environment. Additionally, the package contains more high level and value added features for experimental design and experiment analysis to address the specific need of individual-based models. The underlying implementation idea is to provide a set of turnkey features for facilitating the task of applying the sensitivity analysis to models. Functionally, the package consists in four modules which interoperate together for instantiate and running the Repast code inside R. All these four components

\footnotetext{
${ }^{2}$ Application Programming Interface
} 
are (a) the Repast Integration Broker, (b) the Repast Integration Engine, (c) The R Integration wrapper and finally, (c) the R API for Experiment design. An schematic view of package architecture is shown in Figure 6.3

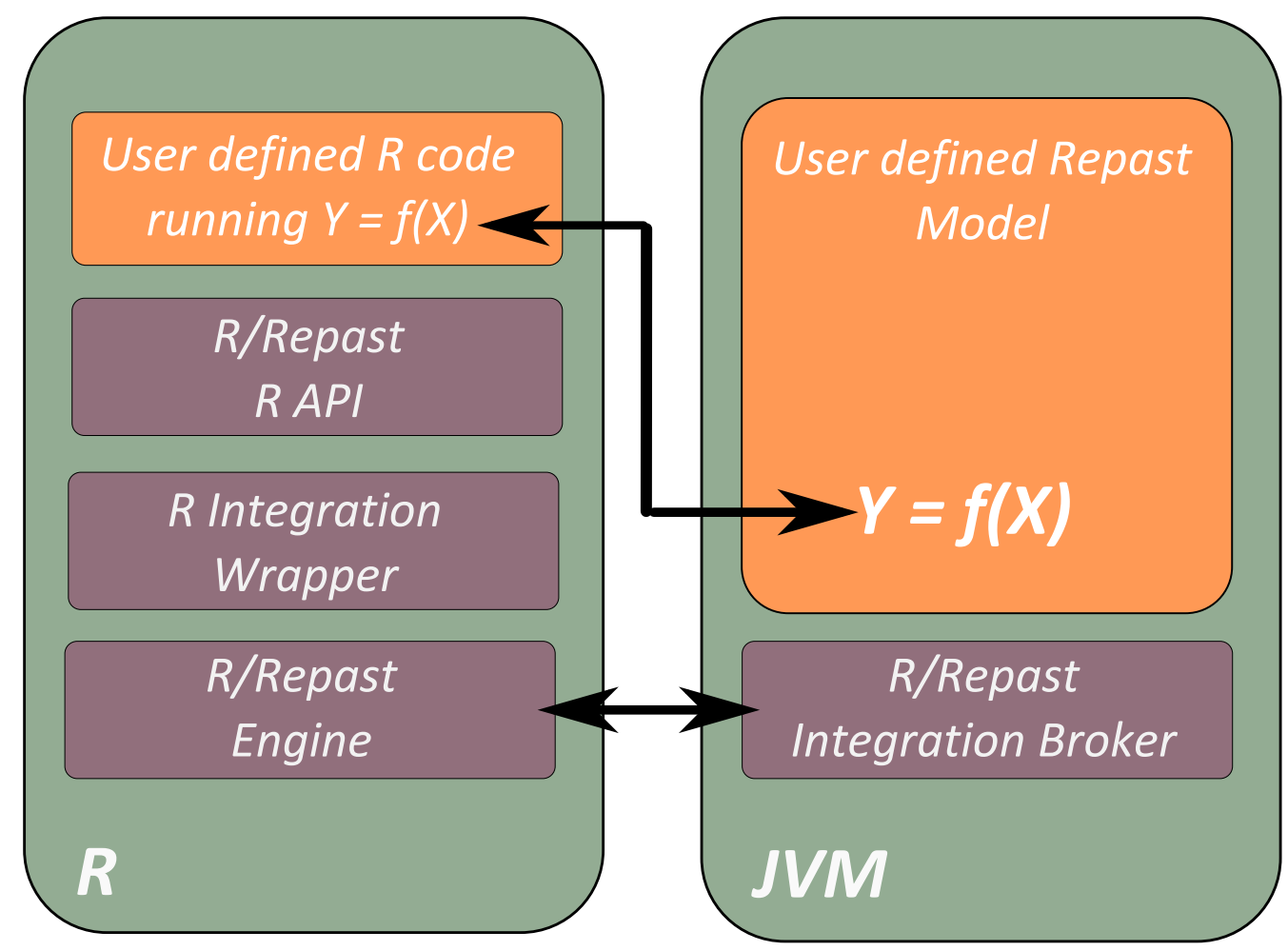

Figure 6.3: The approach for wrapping an individual-based model using the $\mathrm{R} /-$ Repast general architecture. The scheme shows in the left box the $R$ environment and the associated components of $\mathrm{R} /$ Repast. The right box represents the Repast Simphony model running within a Java Virtual Machine as well as the R/Repast integration broker component.

The $\mathrm{R} /$ Repast integration broker and the R/Repast engine are both written in java code and are required for instantiating and loading the Repast Simphony model in batch mode. The R/Repast engine contains also the required hooks for transferring the model output data from Java to $R$ environment. The engine can transfer data from aggregated data set defined by the modeler on the Repast model. An aggregated data set is a Repast Simphony entity used for collect data about the simulation model agents which can be used for plotting or saving the model output data to a file using a file sink. A File Sink is Repast component for saving simulation data to a file. The 
aggregated datasets use some kind of aggregate operation, such as counting, averaging, summing or any other used defined aggregate operation [ $\left.\mathrm{NCO}^{+} 13 \mathrm{a}\right]$, [NCO13c], $\left[\mathrm{NCO}^{+} 13 \mathrm{~d}\right]$. The $\mathrm{R}$ integration wrapper is the $\mathrm{R}$ code for linking together the $\mathrm{R}$ and $\mathrm{Re}-$ past subsystems. This module consist in several wrapping functions for encapsulating the Java code calls implemented using the rJava package [Urb16]. These functions are prefixed with the [Engine] keyword and, although exported in the R/Repast package, they are not intended for general use.

\subsubsection{The R/Repast R API}

The module entitled R/Repast R API is the primary entry point for the user defined code and relies on the subsystems mentioned previously for providing three group of functionalities for facilitating modelers to analyze the simulation output. These group functionalities are the following:

- Execution and control of Repast Simphony code.

- Basic functions for experimental design.

- High level functions for a complete experiment in one call.

The functionalities on the first group are those required for the basic interface between Repast and R system, such as instantiating and running a Repast Simphony model, retrieving the declared model parameters, getting their default values, setting parameter values as well as running basic experimental designs and saving simulation data. The partial list of these basic R/Repast API methods are shown in the following boxes:

\section{Model(d, t, o, 1)}

This function creates an object instance for linking the Repast model to an R object. The required parameters are the directory where the model has been installed $(d)$, the duration of simulation in Repast ticks $(t)$, the name of any aggregated dataset of model for draining data generated by the model simulation (o) and a Boolean flag $(l)$ which tells the function to call the Load method. The default value is FALSE. 


\section{$\operatorname{Load}(\mathrm{m})$}

This function loads the Repast scenario from model's directory. The only required parameter $(m)$ is an instance of Repast Model created with previous function.

\section{$\operatorname{Run}(\mathrm{m}, \mathrm{r}, \mathrm{s})$}

The purpose of this function is to execute a single round of simulation using just one parameter set. The parameters for this function are a model instance $(\mathrm{m})$, the number of repetitions $(r)$ and a collection of random seeds $(s)$ to be used for each one of the repetitions. The only required parameter is the model instance, created with the Model() function. The default value for $r$ is one.

\section{RunExperiment(m, r, d, F)}

Execute a complete experimental setup for different sets of parameters. The parameters required are a model instance $(m)$, the number of replications $(r)$, the experimental design $(d)$ and finally a user provided calibration function $(F)$. The experimental design parameter is an $\mathbf{R}$ data frame containing a complete set of model's parameter per row. The function returns a list with three data frame elements: the paramset, the output and dataset which holds respectively all simulated input parameters, the result of user provide calibration function and the complete dataset produced during the experiment execution.

\section{GetSimulationParameters(m)}

Returns the complete list of parameters declared by the model. The parameter ( $m$ ) is an instance of Repast model generated with Model() call described previously. 


\section{SaveSimulationData( $(t, e)$}

Exports the results of Run or RunExperiment to a csv or excel files. The parameters $t$ and $e$ are respectively the format of exported data (xls or csv) and the experiment results returned by RunExperiment()

The second group of methods within R/Repast R API contain the functionalities required for setting up and applying a complete experimental design to a Repast simulation model. The group include functions for adding the input factors and the relevant input range which the modeler wants to evaluate. The group also have functions for generating the experiment inputs using different sampling approaches. It is not required to add as input factors all declared model parameters, the modeler can just evaluate a small subset keeping the other factors fixed.

The Experimental Setup API functions. These functions are used for experimental design, parameter calibration and sensitivity analysis. The functions of this group are presented below.

\section{$\operatorname{AddFactor}(\mathrm{f}, 1, \mathrm{k}, \mathrm{b}, \mathrm{u})$}

Creates the parameter collection for the experimental setup. The function requires the data frame $(f)$ where parameter will be added, if this parameter is not provided a new data frame will be created. The second parameter $(l)$ is the random function used internally, the default value is runif which will be the valid choice in many cases, the next parameter $(k)$ is the name of factor, the value provided must match some parameter defined in the repast model. The following two parameters $(b),(u)$ are the lower and the upper range, respectively. The function returns the updated $(f)$ data frame with the new parameter.

\section{AoE.RandomSampling $(n, f)$}

Also known as Monte Carlo sampling, generate an experimental design based on making random samplings of parameter space. The function takes two parameters, the sample size ( $n$ ) and the factor ( $f$ ) data frame created using AddFactor(). The function returns the design matrix for the provided parameters. 


\section{AoE.LatinHypercube(n, f)}

Generates an experimental design using the Latin Hypercube stratified sampling technique which is a more efficient sampling scheme, in terms of model evaluations, than the pure random sampling. The parameters $(n, f)$ and return values are the same already described for the function AoE.RandomSampling().

\section{BuildParameterSet(d, p)}

Constructs the data frame required for executing RunExperiment(). The function takes two parameters: the design matrix $(d)$ created with one of previous functions and the declared parameters $(p)$ defined in the Repast Model with the default values retrieved using the function GetSimulationParameters(). The functions returns a data frame with varying and fixed parameters for the experimental setup of choice.

Finally, the third group contains the "Easy" API functions. These functions are intended to provide a complete method implementation which is accessible using just one $\mathrm{R}$ function call. The user has to introduce few initialization parameters such as the directory location where the Repast model is installed, the objective function and the parameters relevant to the specific method. The currently available Easy API methods are shown in the boxes presented below. The objective function is a user defined $\mathrm{R}$ function over the model output for calculating and returning a cost metric for the simulation outputs of interest. The return of objective functions is the target for the application of the analysis method.

The easy API functions. These functions are the preferred entry point for the eventual users. These "Easy" functions lump together a complete experiment task in just one call, reducing the number of lines of code required. 


\section{Easy.Stability $(\mathrm{d}, \mathbf{o}, \mathrm{t}, \mathrm{f}, \mathrm{s}, \mathrm{r}, \mathrm{v}, \mathrm{F})$}

Evaluate the behavior of model output in order to determine the minimum required number of replication of the chosen experimental setup. The function accept the following parameters: the model installation directory $(d)$, the aggregated data source defined within the Repast model (o), the simulation time in Repast ticks $(t)$ which default value is 300 ticks, the input factors to be sampled (f) created with the previously mentioned function AddFactor(), the number of parameter samples $(s)$, the desired number of replications to be tried $(r)$ being the default value 100 , the output variables of interest which will be checked for their stability and convergence of the coefficient of variation $(v)$; if this parameter is leaved empty all output variables are checked and finally the user provided calibration function $(F)$ for determining the best input parameter combination.

\section{Easy.Morris $(\mathrm{d}, \mathrm{o}, \mathrm{t}, \mathrm{f}, \mathrm{p}, \mathrm{s}, \mathrm{r}, \mathrm{F})$}

This function performs all required tasks for carrying out the method of Morris for screening. The parameters are practically the same as described for the previous function with exception of parameters $(p)$ and $(s)$ which are respectively the levels of input factors and the number of sampling points of Morris method [PIwcfSDV ${ }^{+15}$ ].

\section{Easy.Sobol $(\mathrm{d}, \mathrm{o,t}, \mathrm{f}, \mathrm{n}, \mathrm{r}, \mathrm{F})$}

Encapsulate all required steps for performing sensitivity analysis using Sobol method. The method of Sobol is a global sensitivity analysis technique based on the decomposition of output variance ([STCR04, PIwcfSDV +15]). The parameter semantics are the same already described: the model installation directory (d), the aggregated data source defined within the Repast model (o), the simulation time in Repast ticks ( $t$ ), the input factors to be sampled ( $f$ ), the sample size ( $n$ ), the desired number of replications $(r)$ and calibration function $(F)$. 


\section{Easy.Calibration $(\mathrm{d}, \mathbf{o}, \mathrm{t}, \mathrm{f}, \mathrm{n}, \mathrm{r}, \mathrm{F})$}

This function estimates the best set of input parameters or factors, performing a set of model executions in order to sample the calibration function. The objective of this function is to minimize the output of calibration function provided by the user.

\section{Easy.Setup(d,1)}

The parameters (d) and (l) are respectively the directory where repast model is installed and the location of R/Repast deployment directory. If omitted, it assumes as the default value, the directory where the Repast model is installed. The function is required for automatically making the changes in the model configuration for adding the integration code, for deploying the Java jar files with the integration code and for preparing the deployment directory. That directory will hold the JVM logs and the saved model output data sets.

\subsubsection{The objective function interface}

The last piece of $\mathrm{R} /$ Repast architecture is the definition of the objective function which actually allows the flexible definition of the model analysis target decoupling it from the Repast dataset output. As we have mentioned previously, any model is a functional relationship between a vector of input parameters $X$ and a scalar dependent variable $y$ and expressed as $y=f(X)$. On the other hand, usually the dataset collected from Repast model execution will be a time series where the aggregated measure will be collected at fixed intervals. Therefore, some transformation must be applied in order to obtain a value consistent with the functional definition. In addition, even though the value returned from the Repast model were a scalar one, it would add much more maintainable and flexible a to act upon it directly from $\mathrm{R}$ without have to making changes in the Repast code. The objective function is also necessary for calibrating, where the output values are compared to some reference data or even for more complex tasks, such as tuning oscillations in the population output. It is also the place for normalizing he model outputs. The objective function is a required parameter for all methods 
presented here.

The specification of R/Repast requires the objective function having two input parameters. The first of it, which is passed for the objective function is the set of input parameters used for executing the Repast model, the second parameter are the results generated by executing the model and corresponding to and aggregated data set in the Repast model. The objective function must return one or more scalar values grouped using the cbind () [Cra07] R function. The complete function signature is shown in Figure 6.4.

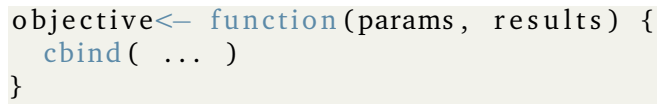

Figure 6.4: The skeleton of objective function. The function has two parameters and must return a one or more scalar values.

\subsection{Examples overview}

In the next sections we will provide examples on how the R/Repast can help modelers on the analysis of their simulation models. Three examples will be used for illustrating the application of some package's functionalities and what kind of information these functions can offer about the simulation outputs. For clarifying what every model does a summary version of ODD will be given for facilitating a general idea about these models. The Overview, Design concepts and Detail (ODD) is a protocol [GBB $\left.{ }^{+} 06\right]\left[\mathrm{GBD}^{+10}\right]$ which has been proposed as a standard way to specify and describe Individual-based models. A brief description on the model structure and parameters will be given in order to allow the readers to understand the kind of questions the model is intended to answer and how R/Repast is can be used for analyzing the model outputs. The last section for each model under the title of Model analysis is not part of ODD protocol but it is included to show the results of running the R/Repast model analysis methods.

The first model used as example here is a spatially explicit individual-based representation of bacterial conjugation using BactoSIM for simulate the plasmid spread on a surface attached bacterial colony[PRP15b]. The example will be used for showing 
the application of Easy.Stability method for finding the number of replications of simulation experiments required for obtaining consistent outputs. The second example is a Repast implementation of the omnipresent predator-prey model describing the interaction between two species. This one is part of examples coming along the standard Repast distribution and will be used for showing the application of Easy.Morris function. Finally, the third example is an instance of the common pool problem in the context of bacterial conjugation. This model was developed exclusively for this work. This model will be used to exemplify the use of Easy.Sobol method. The complete sources for all projects are available respectively in the following locations:

- BactoSIM: https://github.com/antonio-pgarcia/haldane

- Predator-Prey: The sources come with the Repast distribution.

- T4SS Common Pool: https://github.com/antonio-pgarcia/PoolT4SS

For convenience, in order to facilitate the experiments shown in this paper being reproduced elsewhere, we also provide the pre-built installers for the three projects mentioned previously. The installers can be download from URL shown below:

- BactoSIM: http://goo.gl/YYIt10

- Predator-Prey: http://goo.gl/cJ5z2r

- T4SS Common Pool: http://goo.gl/zq4LHO

In order to reproduce the examples that will appear in the next sections, it is required a computer with a Java JVM and GNU R installed. The examples have been produced and tested on a windows box with java 1.8 and GNU R 3.3.1. If these preconditions are met, just proceed to download and install the examples and the R/Repast package. The installation of R/Repast is carried out using the install command install.packages("rrepast") on the R environment. Once the previous steps have been completed, just copy and paste the examples shown in this paper, taking care of changing the references to the model installation directory to the directories where the models have been installed locally. 


\subsection{Example 1: BactoSIM}

Typically, one of the advantages of using individual-based models for biological or ecological processes is the possibility of incorporating variability at an individual level. Therefore, unlike deterministic model, in order to get trustworthy results, the simulation must be repeated a number $N$ of times to achieve stable value on the output variance. The objective of the first example is to show the application of a simple method for finding the minimal number of replications of a simulation model which is required for the variance of response variables become stable, converging to a common value. $A$ straightforward way to determine the output stability has been suggested in [TKG14] [LHM12] and consists in to compute the coefficient of variation 3 of the output of interest with and increasing number of repetitions while keeping the input parameters fixed. The number of replications for which the values of coefficient of variation stop to vary are the minimum number of repetitions necessary for getting robust results. In R/Repast we have implemented that method which is accessible through the Easy.Stability API call.

For this example will be used the BactoSIM[PRP15b] model. This is an individualbased model of bacterial conjugation process. The bacterial conjugation is a form of lateral genetic transfer which occur naturally in bacterial colonies [AF13]. The conjugation consists in the transference of a conjugative plasmid from a donor cell to a recipient cell. The plasmids are small circular DNA sequences which replicates independently from the main chromosome of their hosts [BLL00]. The conjugation is considered one the causes of the rapid evolution and adaptation of bacterial colonies and the spread of antibiotic resistance [CCD05] [SBTT08]. The BactoSIM model is currently being used for an evaluation of the main factors governing the plasmid dispersion. A preliminary evaluation has shown that the point in the cell cycle are the principal factor responsible for the global dynamics of plasmid infective dispersion[PRP15a] which is consistent with some observations[SYD+11] taken from individual bacterial cells. The complete description for the BactoSIM, including the ODD protocol can be found in section 7.2 .

\footnotetext{
${ }^{3}$ Also known as relative standard deviation given by $C V=\sigma / \mu$ which provides a normalized version of the standard deviation expressed relatively to the output mean
} 


\subsubsection{Model analysis}

The objective of stability analysis is to find the minimum number of experimental setup replications required for achieving reliable results. Thereby, the model output response is evaluated for an increasing number of repetitions allowing the evaluation of the convergence for output variance of simulation outputs. The complete listing for carrying out the stability check for the BactoSIM model is shown in Figure 6.5. As can be observed, the complete implementation of model analysis encompasses five steps. These steps are conserved for all high level functions available in $\mathrm{R} /$ Repast package. The step 0 clean all existing $R$ objects, loads the $R /$ repast package and set the random seed for the analysis. The step 1 is the definition of the objective function which can be any user provided function following the R/Repast API specification. It is not strictly necessary for the Easy.Stability as the coefficient of variation is calculated for the model output variables. In this example the objective function is basically the comparison of simulated data and experimental data using the normalized root mean square error API call AoE.NRMSD. The step 2 is adds the model input factors for which the importance on the model output will be assessed and their biologically relevant range of variation. It is necessary to add at least one parameter which will be varied, while all other model parameters are keep fixed using the default value or with a value previously set using the R/Repast API SetSimulationParameter. The purpose of step 3 is to configure automatically the Repast model with the integration broker and for initializing the integration directory. Finally, the step 4 is where the analysis method is invoked, all analysis methods will return a list holding three objects, namely the experiment, the object and the charts. The experiment contains simulation parameters and results, the object is method specific and finally the charts are pre-generated graphs for the method results 4

The method will generate automatically one chart for each model output5. One of the output chart of model is shown in Figure 6.6 for the variable named X. Simulated. As can be observed, the coefficient of variation of these variable decreases as the sample size increases. The variation starts to become acceptable with a sample size of 25 and approximately with sample size of 50 we can see that coefficient of variation

\footnotetext{
${ }^{4}$ In the currently API version there is a function for accessing the charts for the Easy.Stability method, named Easy.getChart(), please refer to the user manual for the complete syntax.

${ }^{5}$ It is possible to limit it passing to the method a subset of model outputs
} 


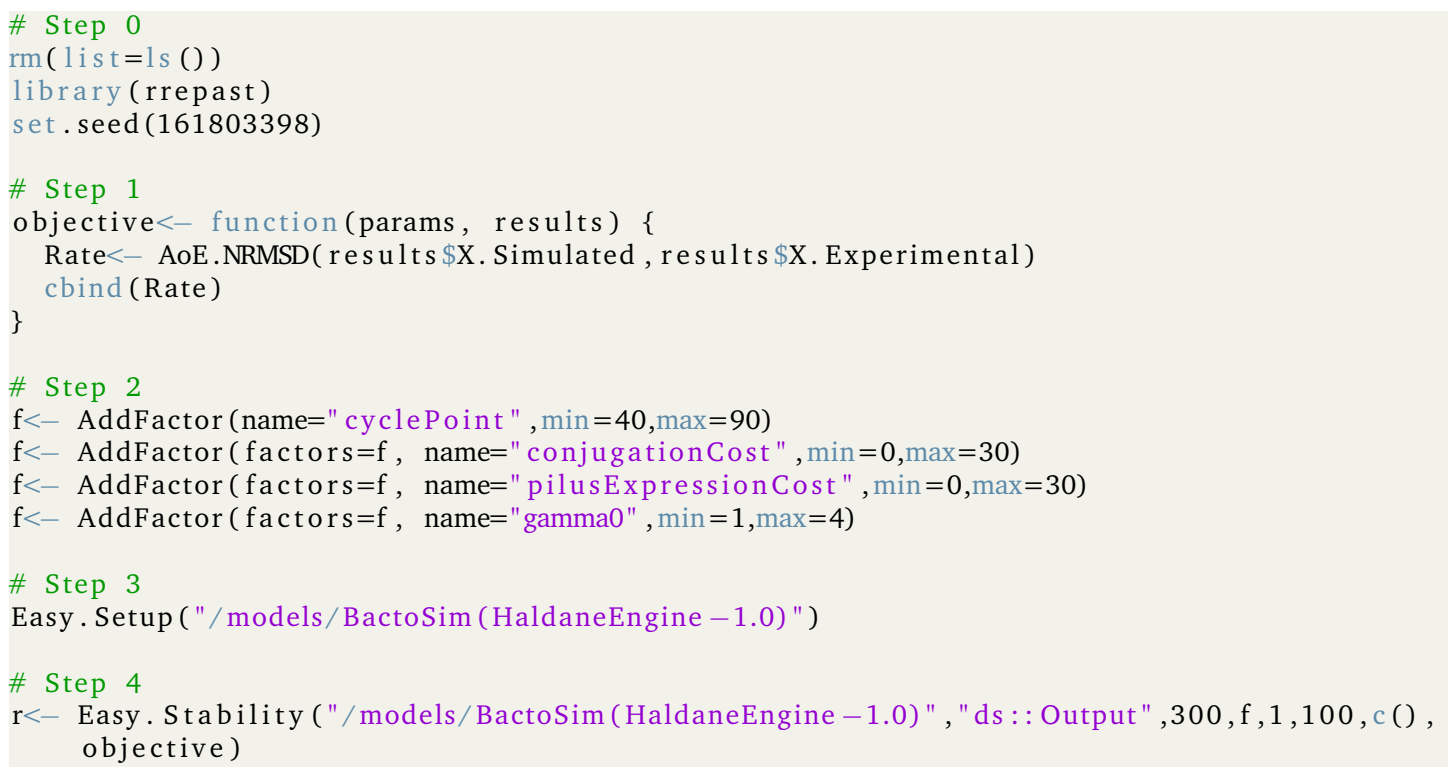

Figure 6.5: The listing for stability of model output method using the Easy.Stability function from R/Repast.

become stable. Therefore, we can feel relatively confident with or model results with a number of replications greater than 25. Certainly, it is important to take into account the computation cost of our model in order to select a value for the number of repetitions.

\subsection{Example 2: Predator-Prey}

\subsubsection{Model description}

\subsubsection{Purpose}

The purpose of Predator-Prey model presented here is to provide and alternative individual based-model implementation for the classic ODE model describing the association between two species. The model will be used to show the application of Morris method for ranking the most important parameters. 


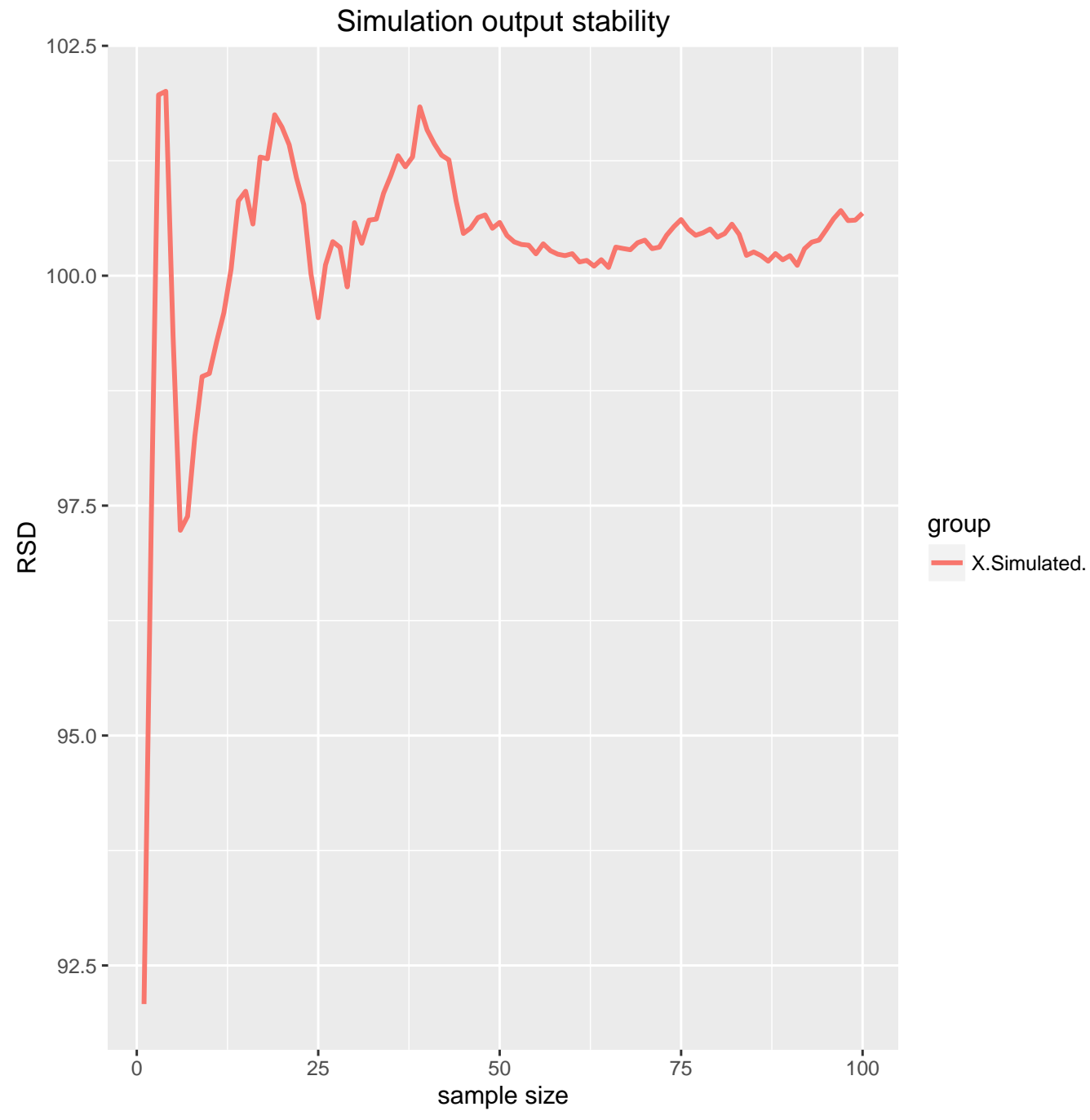

Figure 6.6: The stability of model output. It is possible to observe how, insofar that the number of replications of the experimental setup increases, the value of the coefficient of variation converges to a common value.

\subsubsection{Entities, State variables and scales}

The model comprises three entities or agent types, the wolves, the sheep individuals and the grass. These agents evolve in a computational domain of a $50 \times 50$ units with periodic boundaries, representing a large portion of space. The agents are positioned in a continuous bi-dimensional space and are free to move. On the other hand, the grass agent is placed in a discrete grid. 


\subsubsection{Process overview and scheduling}

The agents are defined by the execution of a set of processes depicting the agent movement and search of food source, the consumption of food, the process incrementing the agent reserves, the reproduction and finally the death process driven by predation or starvation. The fundamental idea behind the model formulation is that both predator and prey individuals incrementing their "energy" levels by predation or by consuming the available grass respectively. Both agent types search for their food in the current patch where they are placed. The agents move a unit of space at time selecting randomly the heading.

The individual-based version of this model is a spatially explicit representation and have a few parameters more but is still very succinct. The list of model parameters are shown in Table 6.1.

Table 6.1: The input parameter collection for the Repast implementation of Predator-Prey model.

\begin{tabular}{ll}
\hline Input parameter & Description \\
\hline $\begin{array}{l}\text { initialnumberofwolves } \\
\text { initialnumberofsheep }\end{array}$ & $\begin{array}{l}\text { The initial population of predators. } \\
\text { The initial population of preys. } \\
\text { The rate of predator energy is incremented every time a } \\
\text { prey is consumed. }\end{array}$ \\
$\begin{array}{l}\text { wolfreproduce } \\
\text { sheepgainfromfood } \\
\text { sheepreproduce } \\
\text { grassregrowthtime }\end{array}$ & $\begin{array}{l}\text { The prey rate energy increment for grazing grass. } \\
\text { The reproduction rate of prey individual. } \\
\text { The amount of time required for grass be available again } \\
\text { once consumed by a prey. }\end{array}$ \\
\hline
\end{tabular}

The original formulation of Lotka-Volterra consists in a system of two differential equations with four parameters, namely the predator and the prey growth rate, the effect of predator on the prey growth and finally the effect of prey on the predator growth as can be seen in Equation (6.8). 


$$
\begin{aligned}
& \frac{d x}{d t}=c_{1} x-c_{3} x y \\
& \frac{d y}{d t}=-c_{2} y+c_{4} x y .
\end{aligned}
$$

There is a conceptual correspondence between the predator $c_{2}$ and prey $c_{1}$ growth rates with the model parameters wolfreproduce and sheepreproduce as well as with between the parameter wolfgainfromfood and the constant $c_{4}$.

\subsubsection{Model analysis}

The implementation code for the Morris screening exercise is shown in Figure 6.7 and, as has been mentioned in the previous example, we have the same sequence of steps, starting with the library loading and the selection of the random seed. Subsequently we define the objective function, which in this case is a very simple one consisting in the arithmetic average of the population sizes of sheep individuals and wolves. The next step is the selection of model input factors for the screening method and providing the range of variation for each them. Then, the step 3 shows the call to the Easy.Setup function which initializes the Repast Model with the R/Repast integration code. Finally, the function Easy.Morris is called and the results stored in the variable $r$. The example uses five levels with ten sampling points for Morris method. The results consist in a $\mathrm{R}$ list holding the experiment carried out, the Morris object and a list with charts generated by the experiment 6 .

The Figure 6.8 presents the $\mu^{*}$ vs $\sigma$ chart for both predator and prey average population sizes. At a first glance, the most important input factor for both Predator and Prey populations is the sheepgainfromfood. The second most significant for the Predator output is grassregrowthtime. The other parameters are not very significant for the average of Predator individuals. It is also interesting to note that wolfgainfromfood has very high value of $\sigma$ which could indicate that the parameter significance strongly depends on the values of other parameters. On the other hand, it could mean that the num-

\footnotetext{
${ }^{6}$ In order to plot the charts the user should use a R code for accessing the chat list members. There are three members, namely mustar, musigma and mumu. In order to get the mumu chart for the second objective function output we must use the R call: $r \$$ charts [2,] \$mumu.
} 


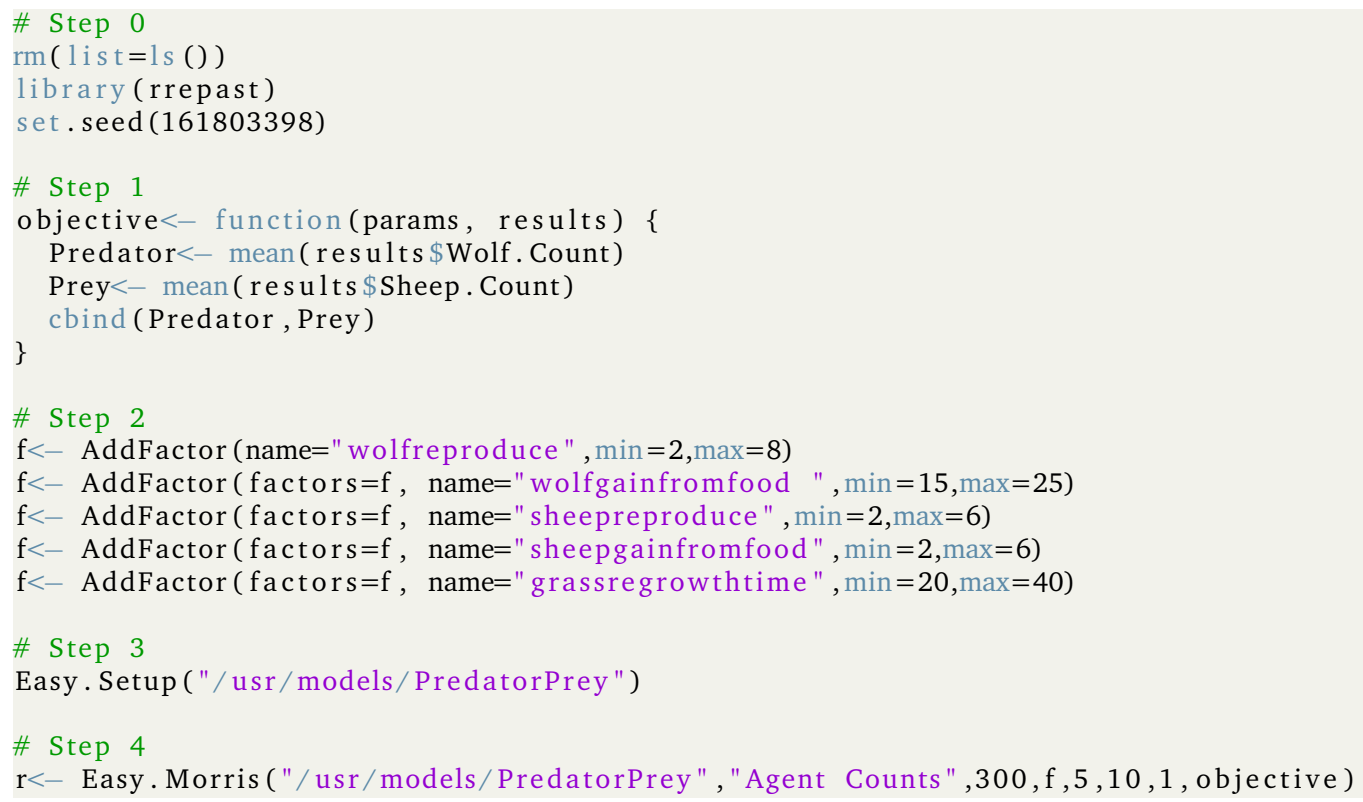

Figure 6.7: The listing for Morris screening method using the Easy.Morris function from $\mathrm{R} /$ Repast.

ber of sampling points or replications should be increased. The Prey output presents three important parameters, which in order of importance are the sheepgainfromfood, the sheepreproduce and grassregrowthtime. These input parameters also have a high $\sigma$ values which possibly indicate some non-linear effects or that the values of these input factors are influencing each other. These results can be explained by the dependence of wolf population on the availability of prey. The common observed pattern in that kind of model is the population of predators lagging in phase behind the prey population.

The chart of $\mu$ vs $\sigma$ for model output is shown in Figure 6.9. It seems to provide very similar results and the only significant difference is the contribution of grassregrowthtime. That input parameter was considered important by $\mu$ vs $\sigma$ but here it has a negative value. In order to interpret this sensitivity measure, we must recall that $\mu^{*}$ takes the absolute values of elementary effects. Therefore, the elementary effects of grassregrowthtime possibly has effect of opposite sings depending on the values of that input parameter.

Finally, we have the 6.10 showing the chart of $\mu^{*}$ vs $\mu$ where the value of both measures can be observed together allowing the appreciation of the differences of 


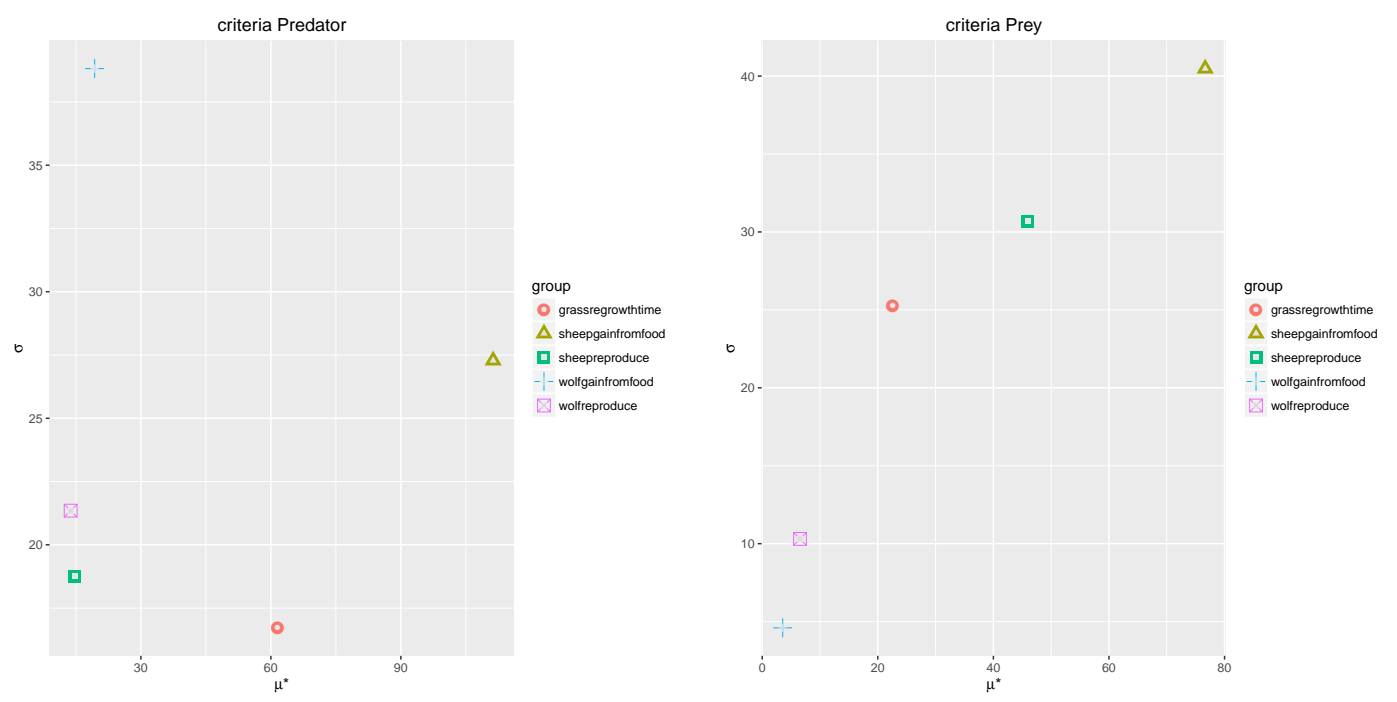

Figure 6.8: Results of Morris screening method for Predator-Prey model. The graph shows the $\mu^{*}$ and $\sigma$ sensitivity measures for Predator and Prey outputs.

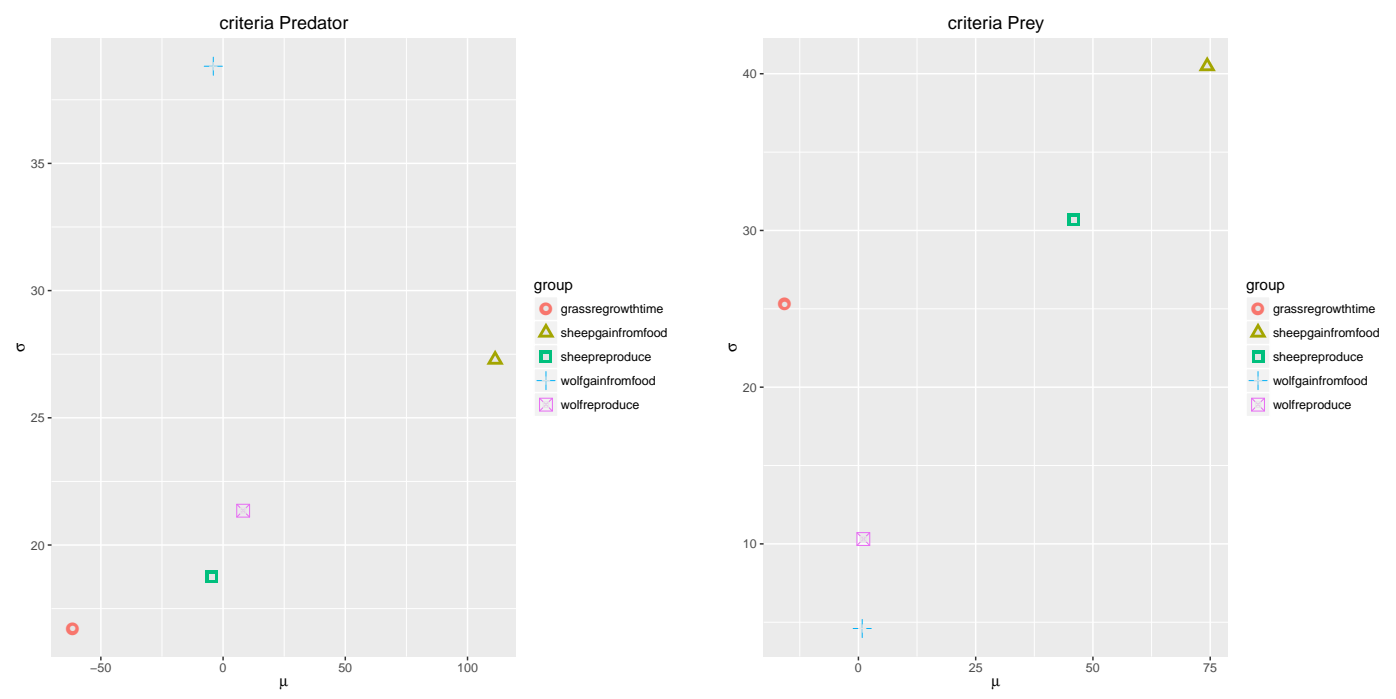

Figure 6.9: Results of Morris screening method for Predator-Prey model. The graph shows the $\mu$ and $\sigma$ sensitivity measures for Predator and Prey outputs.

both, which possibly indicates that the input factors present effects with different signs which, in other words, means non-linearity in the model behavior. 

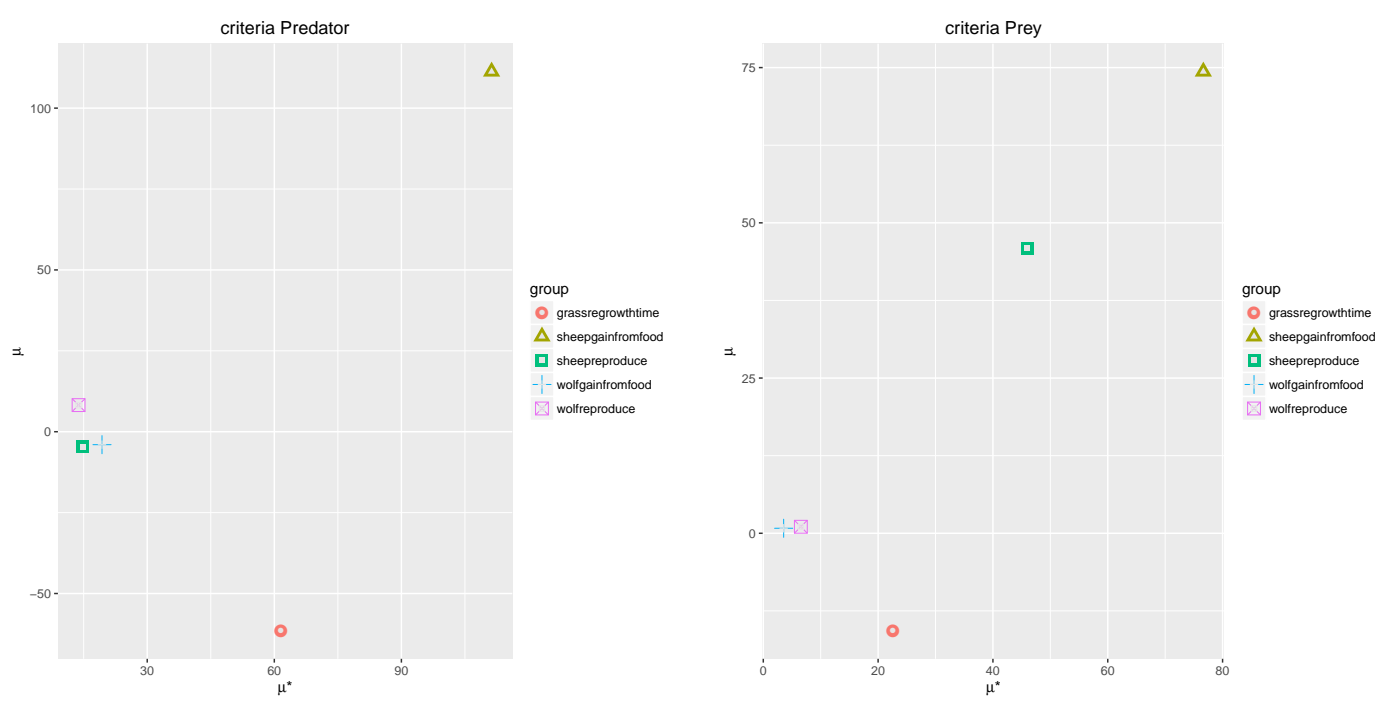

Figure 6.10: Results of Morris screening method for Predator-Prey model. The graph shows the $\mu^{*}$ and $\mu$ sensitivity measures for Predator and Prey outputs.

\subsection{Example 3: T4SS Common Pool}

\subsubsection{Model description}

\subsubsection{Purpose}

The objective of this model is to explore the conditions where two plasmids can coexist in a population competing for a common resource required for their horizontal transfer. The common resource is the set of genes required for conjugation because one of the two plasmids have lost these genes.

\subsubsection{Entities, State variables and scales}

The model uses two types of entities, namely the agents representing the bacterial cells and a ValueLayer, which is a Repast specific structure, for holding the nutrient available for the bacterial growth. The agents interact and grow in a computational domain of $100 \times 100 \mu \mathrm{m}$ squared lattice with periodic boundaries representing a total real surface of $0.01 \mathrm{~mm}^{2}$. Despite of being a lattice the bacterial cells are positioned and allowed to move in a continuous space system. The agents are also allowed to overlap to each other. Explicitly, the agents are defined by the five state variables: (a) 
heading, (b) mass, (c) division mass, (d) plasmid $P 1$ infection state and (f) plasmid P1 infection state. The current position of every bacterial cell in the coordinate system is available implicitly through a Repast API call.

\subsubsection{Process overview and scheduling}

Every bacterial cell in this model is abstracted as the execution of a series of successive processes capturing the basic tenets of bacterial life-cycle. These processes are the nutrient uptake, the bacterial cell growth, the division and the conjugation. The input parameters required for initialize the model are shown in Table 6.2 .

Table 6.2: The input parameter collection for the conjugative plasmid common pool model.

\begin{tabular}{ll}
\hline Input parameter & Description \\
\hline doublingTime & The doubling time of plasmid free cells \\
p1P $\left(P\left(\gamma_{0}\right)\right)$ & The probability of cell conjugate at least one time. \\
p1Cost & $\begin{array}{l}\text { The cost imposed by the plasmid P1 including the meta- } \\
\text { bolic burden required to express the conjugative appara- } \\
\text { tus. }\end{array}$ \\
p2Cost & The metabolic cost of plasmid P2
\end{tabular}

\subsubsection{Design concepts}

Basic Principles - The plasmid dispersion rely upon an intricate balance between metabolic costs associated to horizontal and vertical dispersion strategies. The conjugative proficiency requires the expression of set of transmembrane proteins which are known collectively as Type IV Secretion Systems (T4SS) [LKGF03b]. The presence of conjugative plasmids and the expression of conjugative machinery is detrimental for the host cell fitness [RARE ${ }^{+} 04$ ] but there is no consensus on the valid ranges of metabolic costs imposed by the conjugative process. Therefore, in this model the short term dynamics of two plasmid system $P 1$ and $P 2$ is simulated. 
The plasmid $P 1$ is a complete conjugative plasmid containing all genes required for horizontal transfer and the plasmid $P 2$ is a cheater, which having lost its conjugative genes, depends on the T4SS system from the plasmid $P 1$. In other words, the model is used to assess how large should be the cost difference required for the lack of conjugative apparatus become a true competitive advantage making P2 dominate over $P 1$.

Emergence - The colony growth pattern, the population distribution and the dominance of a plasmid over another on the bacterial population are global properties arising from local properties defining the agent behavior and the interaction constraints.

Adaptation - All agents adapt their growth rate, as well as the conjugation rates, according to the local availability of nutrient.

Fitness - The bacterial cells infected by any plasmid are considered to behave less efficiently than the plasmid free cells. The fitness of plasmid bearing cells are explicitly specified by the cost input parameters.

Objectives - No objectives are taken into account in this model.

Prediction - The model will provide predictions on the possible ranges of plasmid metabolic cost which can favorable to the cheaters plasmid strategy.

Sensing - The agents representing the virtual bacterial cells sense the environment to the extent that the nutrient availability controls the growth and the conjugation rates. Interaction - Bacterial cells interact with their nearby individuals for nutrient access, cellular division, mate pair formation and plasmid transfer.

Stochasticity - Stochasticity is introduced at an individual level for all cellular process sampling a normal deviate and fitting the value to corresponding process.

Collectives - No collectives are taken into account in this model.

Observation - The model provides two kind of outputs, one is numeric and contains the total number of bacterial cells which are plasmid free or are infected by the plasmids $P 1, P 2$ or both. These outputs are generated for every time step. The model also has an 2D view of colony growth updated every time tick. 


\subsubsection{Analysis of model}

The global sensitivity analysis using the Sobol variance decomposition method for the T4SS Common Pool model is shown in Figure 6.11. We can observe the same sequence of steps which has been previously mentioned. The objective function is defined for the average values of the model outputs named P1, P2 and Both. These variable are respectively the bacterial population size infected by the P1 plasmid, infected by the cheater plasmid P2 and finally the number of individuals infected by both plasmids. The Sobol sensitivity indices will provide the measures of the importance of every input parameter shown in step 2 of Figure 6.11 with respect to the results returned by the objective function, that is to say, the average population sizes.

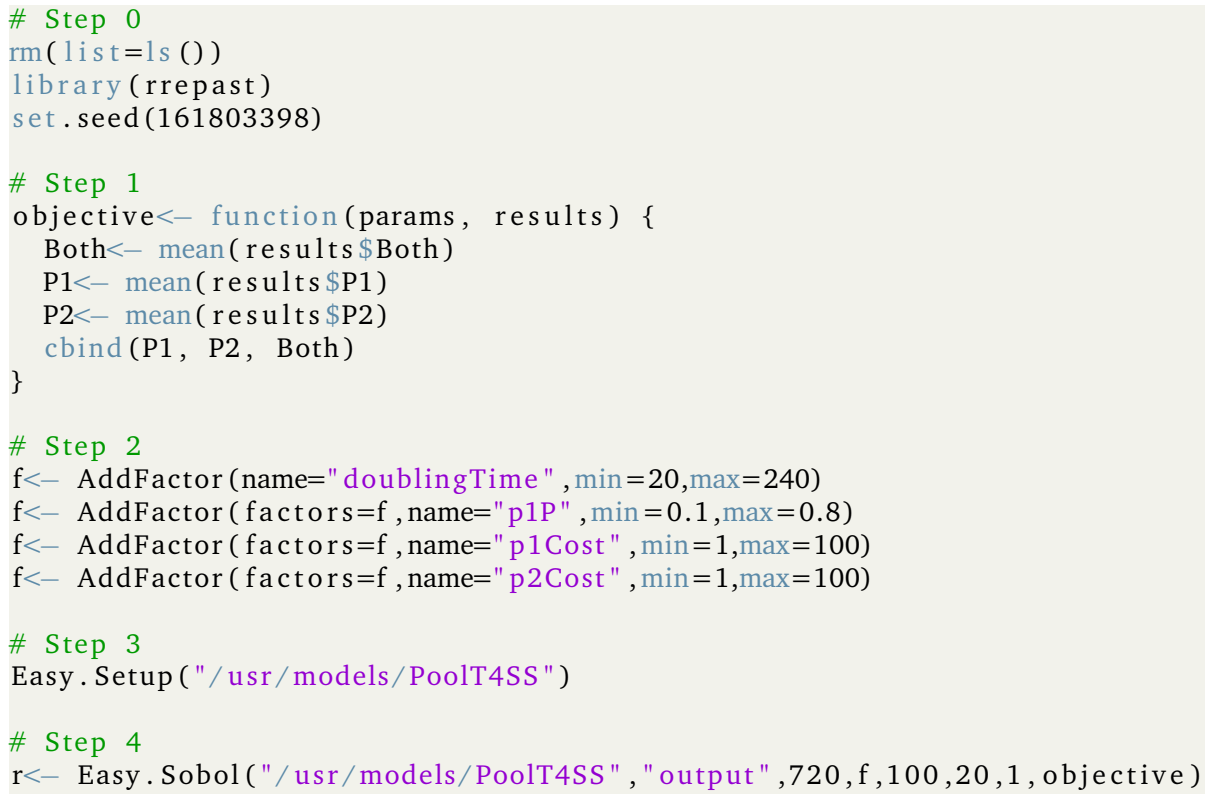

Figure 6.11: The listing for Sobol GSA variance decomposition method using the Easy.Sobol function from R/Repast.

The Figure 6.12 shows the first and total order indices for the model output P1. That output is the average number of bacterial cells infected just by the plasmid P1. As can be observed the most important input parameter is the bacterial cell doubling time followed by the probability $P\left(\gamma_{0}\right)$. This is an expected result because the rule for the conjugative transfer implemented here, have a dynamics linked to the host cell 
cycle and therefore depends on the bacterial state with respect to the total elongation and the division point[SYD+11], being consistent with other studies where the timerelated parameters are the most important ones[$\left[\mathrm{MLS}^{+} 11\right]$. Other interesting aspect to note is the negative values of first order indices. Obviously the sensitivity indices should not be negative. This is consequence of a small sample size and to correct the problem we must increase it. The other important input factors for the plasmid P1 output are, in order of importance, the probability $P\left(\gamma_{0}\right)$, the cost of plasmid P2 and the cost of plasmid $\mathrm{P} 1$, both with similar sensitivity indices.
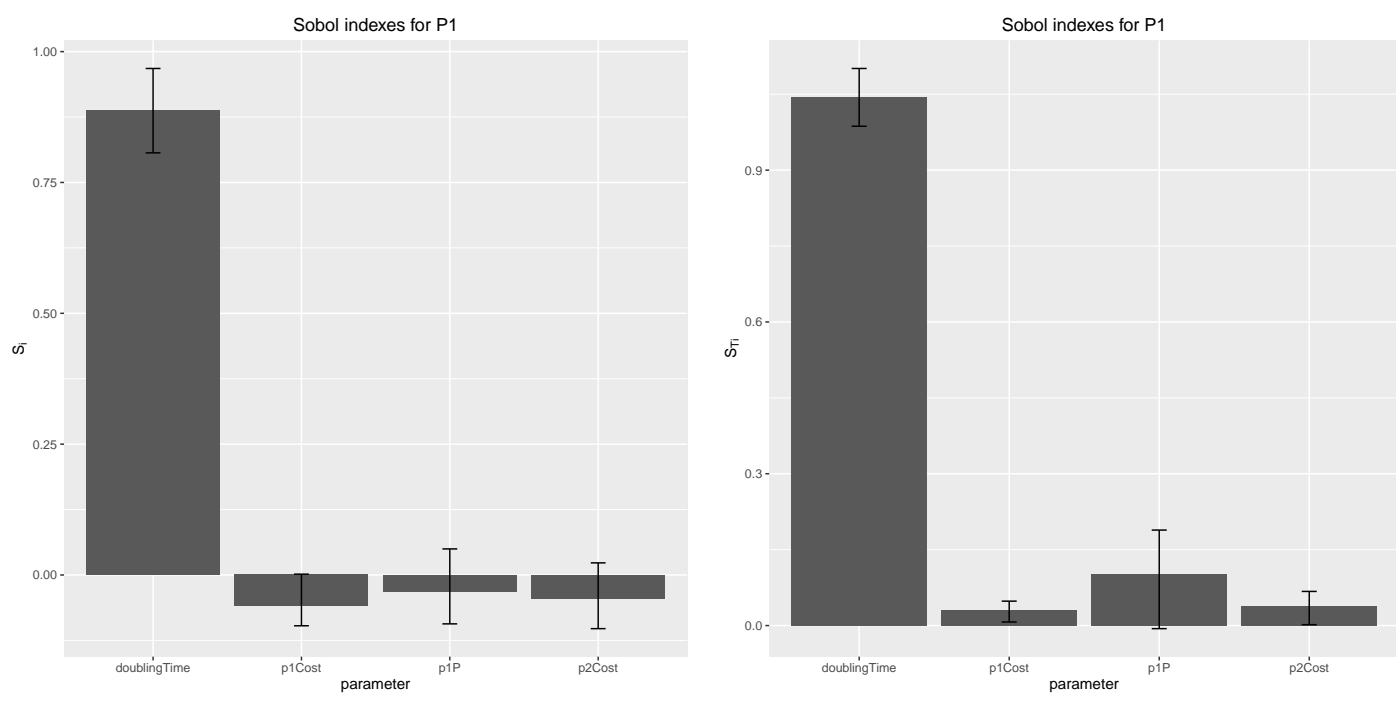

Figure 6.12: Results of Sobol variance decomposition method for T4SS Common Pool model. The graph shows the first and total order indices sensitivity measures for bacterial population infected by plasmid P1.

The first and total order indices for the model output showing the average population size of plasmid P2 can be seen in Figure 6.13. It is possible to appreciate again, that the sensitivity indices show that the most important factor is the length of cellular cycle. The reason is simple, and can be attributed to the fact that plasmid P2 alone is only transferred vertically and depends on the plasmid $\mathrm{P} 1$ for horizontal transmission, being both aspects related to the cell cycle. Following in importance the doubling time we have the cost of plasmid $\mathrm{P} 1$, the cost of plasmid $\mathrm{P} 2$ and the probability $P\left(\gamma_{0}\right)$, being the sensitivity index of $\mathrm{P} 1$ cost, noticeably higher than the other two indices. This could be attributed probably because the plasmid $\mathrm{P} 2$ require a significant cost difference in order to outcompete the plasmid P1 with just the vertical transfer component. 

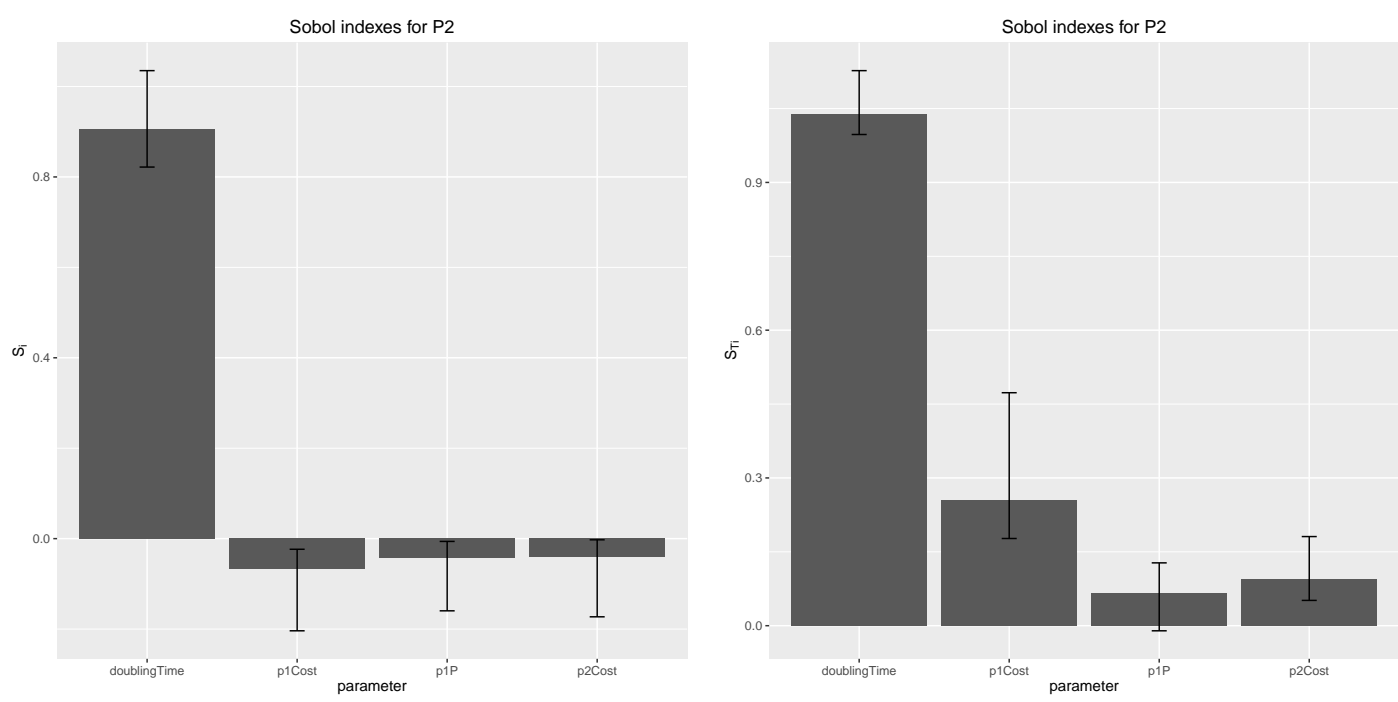

Figure 6.13: Results of Sobol variance decomposition method for T4SS Common Pool model. The graph shows the first and total order indices sensitivity measures for bacterial population infected by plasmid P2.

Finally, in the Figure 6.14 we have the sensitivity indices for the output of model accounting for the average population size of bacterial cells infected by both plasmids. The importance of factors is consistent with the explanations for the previous sensitivity indices. Again, the most important model parameter is the doubling time of bacterial cell followed by the P1 and P2 cost parameters and by the probability $P\left(\gamma_{0}\right)$.

\subsection{Summary}

The ecological modeling is a complex subject which can be normally perceived as being simpler than it actually is. Specifically, the Individual-based models are subject to many levels of uncertainty, which means that it is hard to get completely fixed the values of model inputs, the model structure and the outputs. Normally there is no complete experimental or observational data to construct mechanistic descriptions of individual and therefore many assumptions and simplifications must be made in order to implement a model. The same is truthful regarding the input values, which are particularly critical in the case of the ecology of microorganism, as normally just very few input parameters are directly observed and the most of them are estimated from whole population experiments. Therefore, it is always important to bearing mind that 

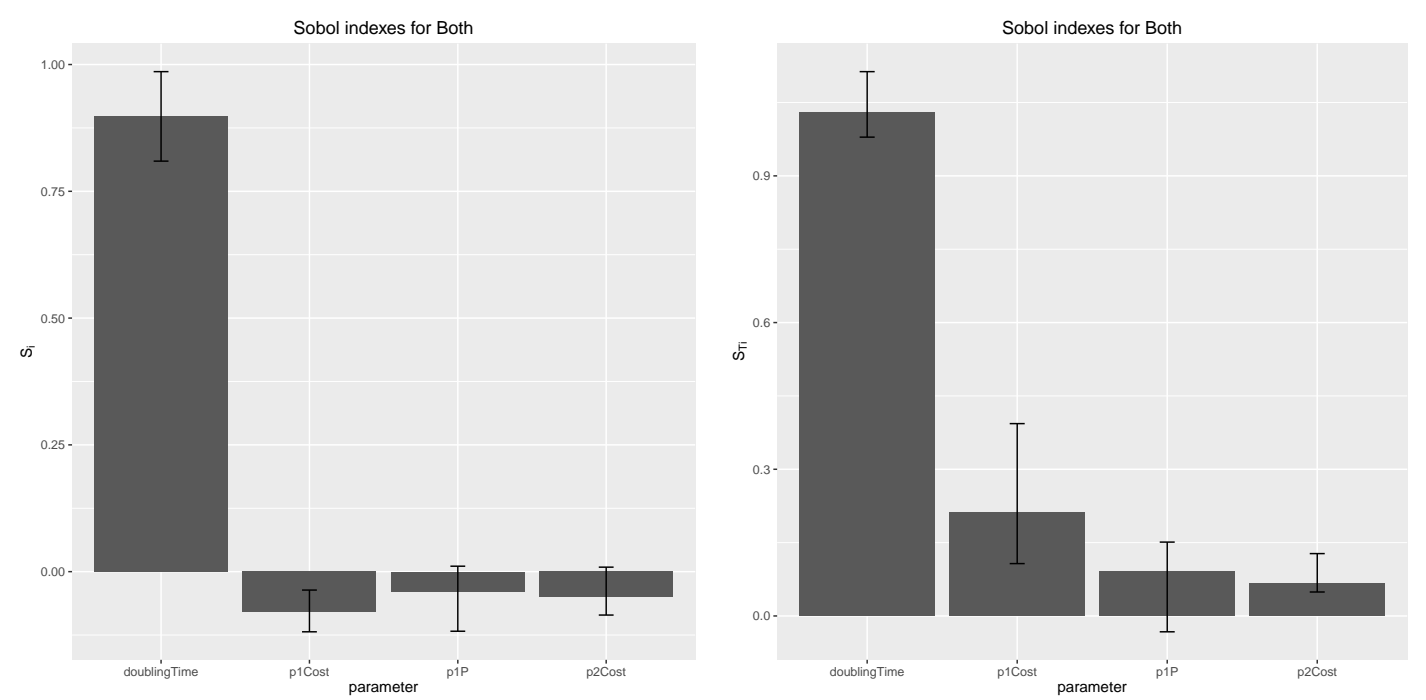

Figure 6.14: Results of Sobol variance decomposition method for T4SS Common Pool model. The graph shows the first and total order indices sensitivity measures for bacterial population infected by both plasmids P1 and P2.

modeling is an iterative task which must incorporate compulsorily some what-if analysis of model outputs.

Several methods exist for assessing the uncertainty and for estimate the relative importance of input parameters in the model output. We have provided here and overview on those methods which are based on the variance decomposition because they have a wider application scope and are specifically suitable for their use on individualbased models. These methods, although conceptually simple, are computationally intensive and can be somewhat hard to apply because the required tools are either unavailable or they do not provide an easy integration pattern. Roughly speaking, the sensitivity analysis methods require the generation of large sample of the parameter space and the model evaluation for each of them which, of course, makes the manual execution an infeasible option.

The in silico experimentation is becoming a vital tool for understanding complex phenomena in a way that cannot be done without modeling. The effective application of computational ecology methods requires a high level of proficiency in many diverse domains of knowledge which sometimes are neither feasible nor practical. Therefore, it is indispensable to have a ready to use arsenal of reusable computational tools for modeling and analysis. In this work we have introduced the R/Repast package and 
shown how it can help modelers to improve the robustness and quality of individualbased models results by using the functionalities inside the package for analyzing systematically the model outputs. The package can save a lot of effort for modelers by providing simple wrappers for complex methods within a simple and consistent API. We hope that these R/Repast functionalities can facilitate dramatically the systematic analysis of Individual-based models implemented in Repast. 


\section{Chapter \\ An individual-based model of bacterial conjugation}

7.1 Introduction . . . . . . . . . . . . . . . . . . 152

7.2 High-level model description. . . . . . . . . . . . . . . . 153

7.3 Material and methods $\ldots \ldots \ldots \ldots \ldots \ldots$

7.3 .1 Purpose . . . . . . . . . . . . . . . . . . . 157

7.3 .2 State variables and scales. . . . . . . . . . . . . . . 157

7.3 .3 Process overview and scheduling . . . . . . . . 158

7.3 .4 Design concepts . . . . . . . . . . . . . . . . . 158

7.3 .5 Initialization . . . . . . . . . . . . . . . . 161

7.3 .6 Sub-models . . . . . . . . . . . . . . . . . . . 163

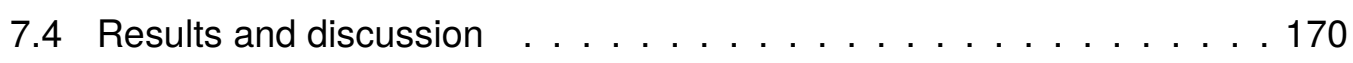

7.5 Summary . . . . . . . . . . . . . . . . . 178 


\subsection{Introduction}

In this chapter we will provide a brief introduction to the BactoSIM simulation platform which we have used for studying the cell to cell dynamics of the horizontal genetic transfer. The main objective of the software is serving in a future as a computational workbench for using bacterial plasmid as a "wiring protocol" for harnessing the power of bacterial cell-cell communication as a tool for multicellular synthetic biology. But we claim that for achieving such goal it is necessary first to understand better the underlying cellular mechanism which promotes the plasmid epidemic spread in a bacterial colony. We have tried to accomplish that goal using an integrative approach where molecular level and individual-based observations are incorporated, sometimes very schematically, into the simulation model. Therefore, all relevant data about the system under study are part of this model as an input parameter or as the guidance and the reference for implementing the internal individual rules for updating the state variables. It is worth to mention that we have been very parsimonious for adding complexity to the model and this representation is the result of several previous attempts.

The construction of an agent-based model uses a bottom-up approach where we specify how agents must evolve by implementing the rules for updating their internal state variables which additionally are also taking into account the stochasticity inherent to biological processes. The whole-population outcomes are a result of these local rules which generates an emergent global behavior. That kind of models is also useful for producing new insights about the process being studied, suggesting sometimes counter intuitive ideas about what local processes are responsible for some global system comportment. The simulation software was developed using the Repast Symphony multi-agent simulation platform $\left[\mathrm{NCO}^{+} 13 \mathrm{~b}\right]$.

The spatially explicit population dynamics models can be classified taking into account the scheme used to describe the population size, space and time [Ber02], thus IBMs uses discrete representations for these three variables. Bearing these ideas in mind we have built a spatially explicit IBM to explore the behavior of conjugative plasmids trying to elucidate the local parameters of conjugal activity and the effect of growth in the plasmid invasion. The spatially explicit individual-based model are par- 
ticularly adequate to generate bottom-up models for microbiological systems in both natural and laboratory conditions accounting for the spatial heterogeneity and furthermore allowing linking the population or system-level properties to the individual parameters helping in the realization about how such global system properties are emerging from the local and individual interaction rules [HB09].

One of the most important aspects shaping the conjugative dynamics is the timingrelated factors according to the results shown in previous studies[MLS $\left.{ }^{+11}\right]$ where the growth dependence is explored as an explanatory reason for the limited invasion in structured colonies. The same idea is also proposed in works about the end-point method [? ZDF+12] which supports that it is impossible to separate the effects of growth and conjugation on the plasmid invasion. Finally the spatial structure itself impose some limitations on the number of reachable neighbors to accomplish a conjugative transfer adding a delay over the propagation front due to the recovery and maturation periods required to forward the plasmid.

The BactoSIM code and binaries are available for download1 The simulator is a part of the European project PLASWIRES which also includes the simulator BactoSIM II[BSRP15]. BactoSIM will serve as the base for an agent-based platform for simulating the conjugation in spatially structured bacterial populations, which are the conditions typically found on naturally occurring colonies such as biofilms or in agar-based laboratory cultures. The model provides a set of key indicators which can be visualized in real time as the simulation evolves and saved for further analysis. The main idea behind this simulator is to provide a simple workbench for verifying several aspects related with the most suitable computational representation for the conjugative process in order to produce a simple and usable operational model.

\subsection{High-level model description}

The simulation model is comprised by a discrete set of agents each of them representing a single bacterial cell $\beta_{i}, i=1 \ldots N$ where $N$ is the current population size at the simulated time $t$. The agents interact with other agents and with the environment which

\footnotetext{
${ }^{1}$ http://goo.gl/TDGxNr
} 


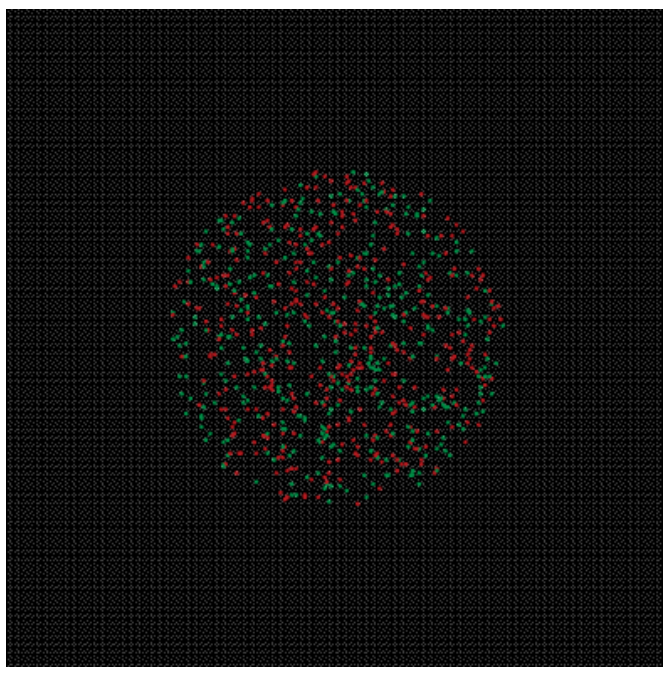

(a) Initialization.

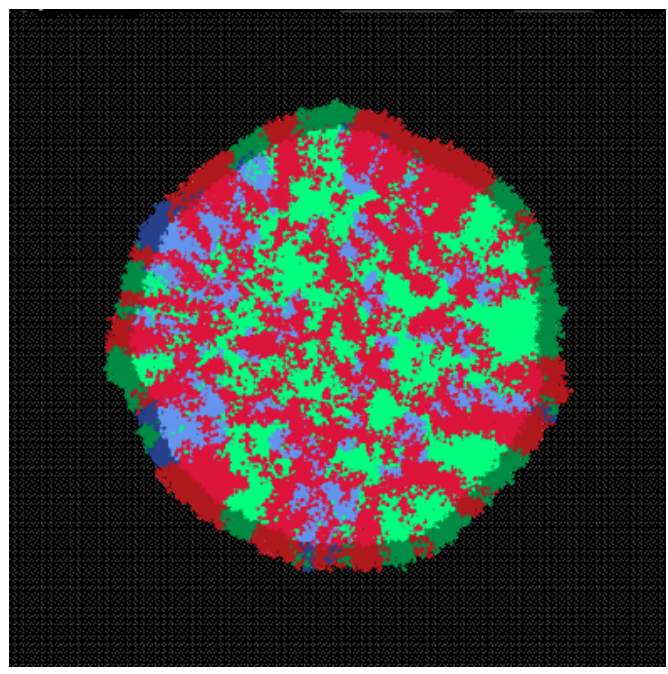

(b) $t=600$ minutes.

Figure 7.1: The BactoSIM Virtual Agar-Plate view for simulated version of $R 1$ plasmid. The color scheme used is green for $R$ cells, red for $D$ cells and blue for $T$ cells. The light-green, light-read and light-blue colors are used to represent depleted nutrient zones where cells are no longer dividing.

holds some amount of nutrient. The environment is implemented using a value layer of Repast framework [ $\left.\mathrm{NCO}^{+} 13 \mathrm{~b}\right]$. The $\beta_{i}$ agents lives and evolve in a computational domain represented as a $1000 \times 1000{\text { discret }{ }^{2}}^{2}$ grid, corresponding to a real surface of $1 \mathrm{~mm}^{2}$. In approximately 20 minutes of wall clock, BactoSIM is able to simulate 600 minutes of colony growth with a final population of $10^{5}$ bacterial cells.

The bacterial cells evolve during the simulated time having their state variables updated by some processes representing the intra and intercellular behavior of every agent $\beta_{i}$. Hence, at a functional high level, the model have a set of processes $\mathcal{P}=\left(p_{1} \ldots p_{5}\right)$ each of them standing respectively for the Uptake, Diffusion, Division, T4SS expression (a protein needed for plasmid conjugation), Shoving relaxation and finally the Conjugation. The execution of these processes is shuffled in order to avoid any bias and the state of agents and the value layer holding the nutrient particles are updated asynchronously. The bacterial cells have three different states, namely $R, D$ and $T$ standing respectively for plasmid free (or recipient cells), plasmid donors (cells

\footnotetext{
${ }^{2}$ Actually it is implemented as a repast multiOccupancy $2 \mathrm{D}$ allowing cells to overlap in some extent, in the same way it really happens in a real bacterial colony
} 
originally infected by plasmid) and transconjugant cells which are those plasmid free cells that have been infected by a donor or transconjugant cell.

The model requires the input of two groups of parameters, the first group is related to the definition of virtual plasmid-host features which will be simulated and the second group allows the specification of the initial population sizes. The first group includes the parameters $G$ which is the doubling time for plasmid free cells $3^{3}$, the point of cell cycle where conjugation is deemed most prone to happen[SYD+11], the T4SS expression and the conjugation cost. The value of $\gamma_{0}$ must be also introduced, that parameter tells the model how many conjugations, on average, are performed by bacterial cells. Model requires also two flags which allow the definition of repressed and conjugative plasmids.

The second group of parameters includes the value of $N_{0}$ or the initial population size which must be introduced in the model as a concentration in cells $/ \mathrm{ml}$ and the initial proportion of donor cells as a percentage of $N_{0}$.

Besides of these two groups the model has an optional parameter for a polynomial equation fitted to the experimental data which serve as a simple visual assessment of the quality of simulated data, an example of this can be seen in Figure $7.2 \mathrm{a}$.

The model generates several outputs with both quantitative and qualitative data about the evolution of the agents and the colony global outcomes. With respect to qualitative data a sample output is show in Figure 7.1 which represents, in some extent, a virtual agar-plate where bacterial agents interact and evolve during the simulated time.

The virtual-plate provides a simple visual tool to assess whether the colony is growing in a realistic way. Besides of the virtual "plate view", BactoSIM generates other six performance indicators summarized in Figure 7.2 the $T /(T+R)$ rate in Figure $7.2 \mathrm{a}$, the population size and the doubling time in Figure $7.2 \mathrm{~b}$ and $7.2 \mathrm{C}$, the $\gamma_{0}, R_{0}$ (Basic Reproduction Number) and the ratio of new infections caused by horizontal transfers in Figures $7.2 \mathrm{~d}$, $7.2 \mathrm{e}$ and $7.2 \mathrm{f}$ respectively. These sample output have been generated using the experimental data for the plasmid pSU2007 with an initial donor density of $5 \%$. As can be easily observed, the model produce structurally consistent behaviors as expected, for instance the generation time of donors and transconjugants are greater than the plasmid free cells which is an emergent property derived from the application of two metabolic costs.

\footnotetext{
${ }^{3}$ The values of doubling time for $D$ and $T$ cells are both emergent properties of model
} 


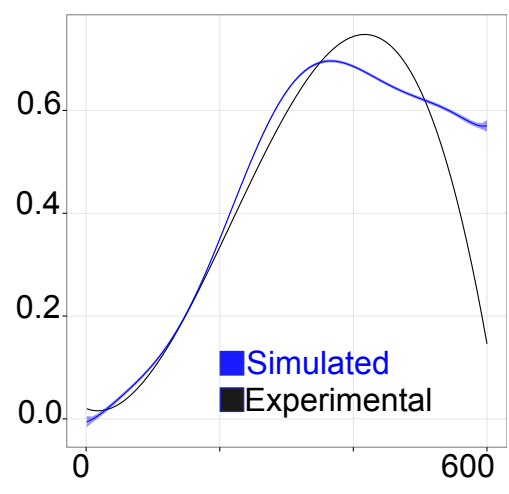

(a) $T /(T+R)$

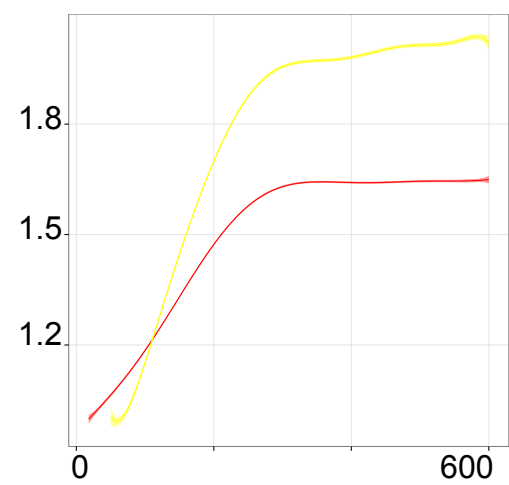

(d) $\gamma_{0}$

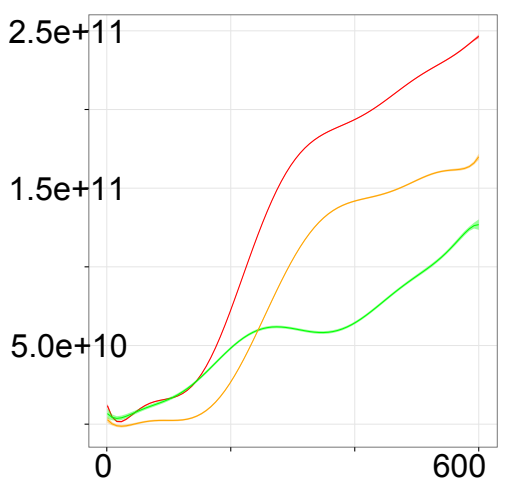

(b) Population

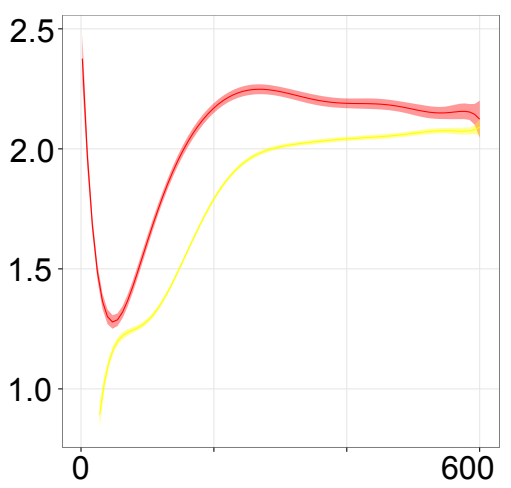

(e) $R_{0}$

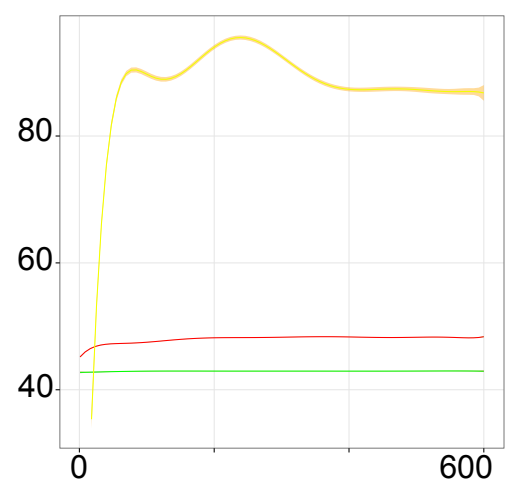

(c) Doubling time

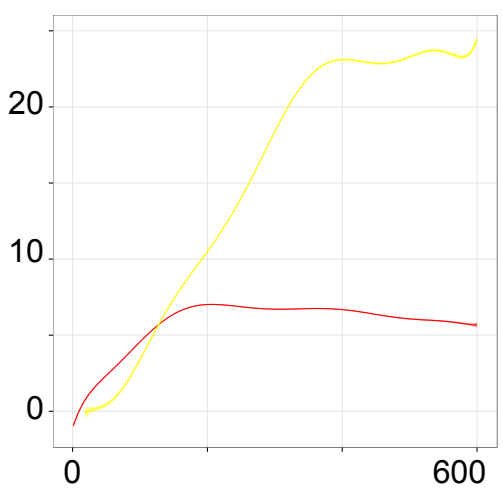

(f) \% Horizontal

Figure 7.2: Sample of the output provided by the BactoSIM model. The subfigure (a) shows the conjugation rates of simulated and experimental populations expressed as the ratio $T /(T+R)$. The subfigure (b) represents the population of different cell types scaled for expressing a concentration in cells $/ \mathrm{ml}$. The next subfigure (c) provides the generation times for recipient, donor and transconjugant cells. The figures (d) and (e) are respectively the so called intrinsic conjugation rate and the basic reproductive number for donors and transconjugant cells. Finally, the subfigure ( $f$ ) is the contribution of donors and transconjugants which can assigned to the conjugative transfer in the creation of infected individuals. The color code used here is green for plasmid free recipients (R), red for initially infected donors (D) and yellow for transconjugant cells (T). The horizontal axis of all subfigures are the simulated time in minutes. 


\subsection{Material and methods}

In this section we present a comprehensive description of our model using the ODD (Overview, Design concepts and Detail) protocol [GBB+06, $\left.\mathrm{GBD}^{+} 10\right]$ which is becoming a standard de facto for describing agent-based and individual-based models in many domains. The model was implemented entirely in java language using Repast Simphony agent-based simulation framework $\left[\mathrm{NCO}^{+} 13 \mathrm{~b}\right]$.

\subsubsection{Purpose}

The main objective $\epsilon^{4}$ of this model is the assessment of what strategy/model type for capturing the structure of conjugation rule, provides the best fit to experimental data and better captures the real structure of the process. Hence, a structurally simple model has been implemented using three different approaches for describing the process of infecting a plasmid free cell. The model results have been compared to the experimental data obtained from wet-lab for assessing the quality of these three strategies. In further works the best strategy can be used to build models which could serve as a predictive tool for synthetic biology and to explore some aspects which are hard to observe directly in experimental studies of plasmid spread. The key points of our model lie on the idea of the existence of a local or intrinsic conjugation rate that has been termed $\gamma_{0}$ which stands for the number of plasmid transfer events, or conjugations on a cell life-cycle basis and that the infection wave speed depends directly from the point along the cell cycle when the conjugative event is triggered.

\subsubsection{State variables and scales}

The model comprises two entity types, namely the bacterial individuals or agents and environment. The environment contains the rate limiting amount of nutrient particles required for the cell metabolism and growth. All agents evolve in a computational domain defined by a $1000 \times 1000 \mu m$ squared lattice divided in $10^{6}$ cells of $1 \times 1 \mu \mathrm{m}$ representing a real surface of $1 \mathrm{~mm}^{2}$. In this model the agents that represents the bacterial cells are defined individually by two main state variables, namely the plasmid infection state

\footnotetext{
${ }^{4}$ The model provide several outputs allowing to evaluate also other aspects of the bacterial conjugation dynamics such as the relative importance of the horizontal transfers.
} 
and the $t_{0}$. The plasmid infection states are $\mathcal{Q}=R, D, T$ and the respective transition function for conjugative plasmids, $\delta$ is shown in (7.1). For the oriT construction only the first transition rule applies since transconjugant cells are sterile. The $t_{0}$ is the time of cell birth or the time of the last cellular division, it is employed in the estimation of agent doubling time used in the division decision rule. The T4SS pili is also taken into account and the agents have a state variable representing the number of pilus already expressed and available in cell surface.

$$
\delta=\left\{\begin{array}{l}
(D, R) \rightarrow(D, T) \\
(T, R) \rightarrow(T, T)
\end{array}\right.
$$

Finally the environment will hold the initial nutrient concentration for every lattice cell. In the model initialization, a fixed amount of substrate particles will be distributed evenly over all lattice sites.

\subsubsection{Process overview and scheduling}

The dynamics of bacterial conjugation is modeled as the execution of following set of cellular processes: the cellular division, the T4SS pili expression, the shoving relaxing which avoid bacterial cells to overlap and allow a more realistic colony growth and the conjugation process. The state variable update is asynchronous. The order of execution of this process is shuffled to avoid any bias due to a purely sequential execution of model rule base, see 7.3. The conjugation process is modeled in three different ways with respect to the time when conjugation event is most prone to happen and the results are compared. Thus the conjugation is defined by to variables: the value of intrinsic conjugation rate $\left(\gamma_{0}\right)$ which determines how many transfers should be performed by a single bacterial cell and the cell cycle point which defines the time when the conjugative events must occur.

\subsubsection{Design concepts}

Basic Principles - The model incorporate some fundamental ideas, which can be axiomatically assumed for all biological system. Therefore, the intercellular competition for nutrients and space is captured by the rules for updating the state of the individual agents. The intracellular competition for metabolic resources sequestered 


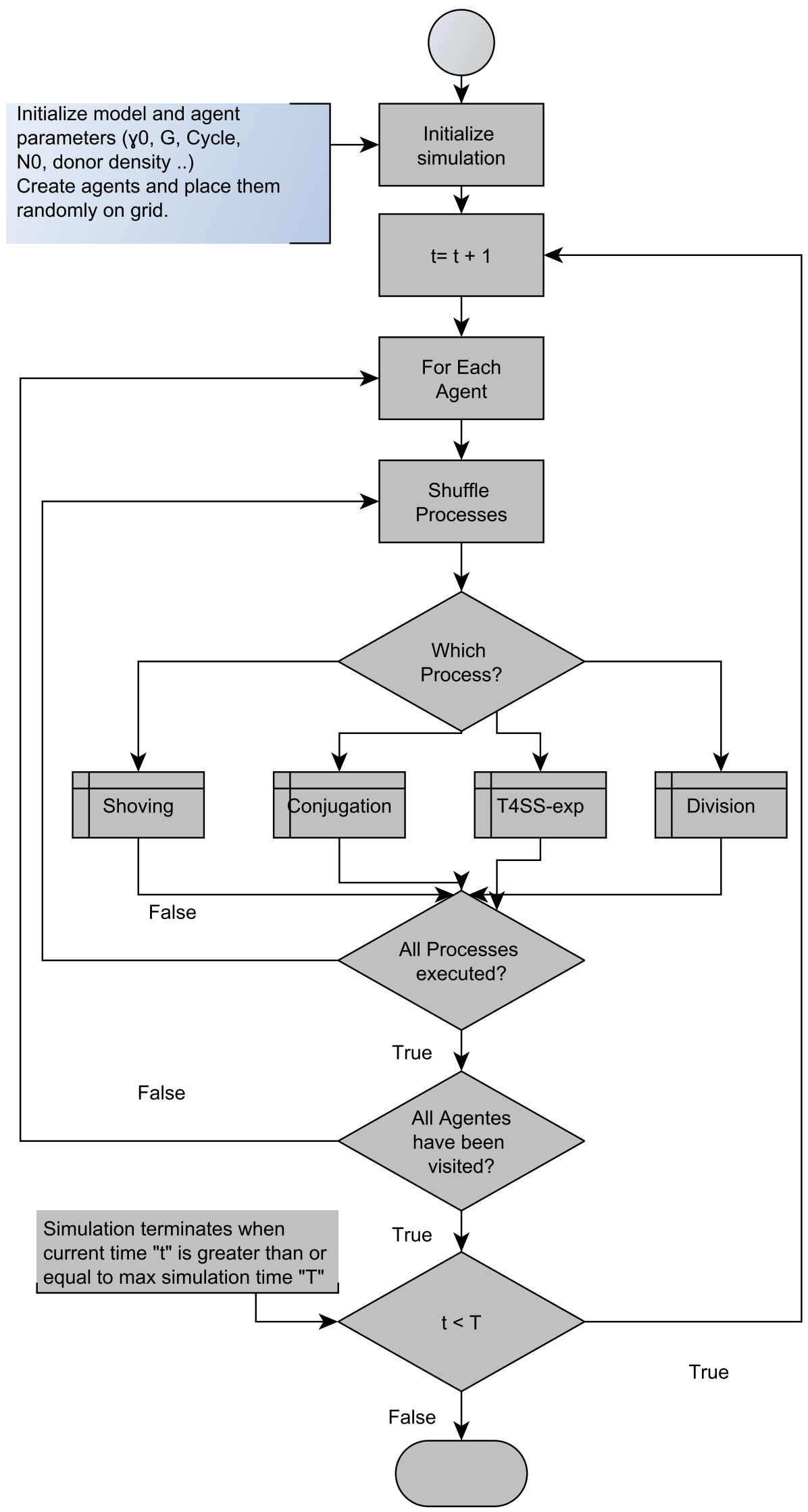

Figure 7.3: The general schematic view for the process scheduling of BactoSIM. 
by the plasmid housekeeping is also taken into account as the associated costs incorporated in the model logic. Three alternative model formulations differing in the way the conjugation rule is implemented and their results compared to the available experimental data. The best strategy can be used to build models which could serve as a predictive tool for synthetic biology and to explore some aspects which are hard to observe directly in experimental studies of plasmid spread. The key points of this model lies on the idea of the existence of a local or intrinsic conjugation rate, which has been termed $\gamma_{0}$. This intrinsic rate stands for the number of plasmid transfer events, or conjugations on a cell life-cycle basis. In addition, the global infective wave speed depends directly from the specific point in the bacterial cell cycle when conjugative event is triggered.

Emergence - We want to find out what will be the global outcome which will arise as function of local rules defining the evolution of the bacterial cells and the interaction with other adjacent cells. With this objective, the model incorporates the most significant aspects of the structure and possible behavior of the structure as cellular processes that are interrelated. Specifically the values of the generation time of donor and transconjugant cells are one emergent property depending fundamentally from the metabolic penalizations applied both for conjugation and for the expression of T4SS genes.

Adaptation - All agents adapt their growth according to the local availability of nutrient and space. It is considered implicitly to the extent that plasmid free individuals will present a better adaptation in terms of growth rate than plasmid bearing cells. Furthermore in one of the model executions we explicitly impose a fitness penalty every time that conjugation occurs in order to compare the resulting bacterial colony state.

Prediction - The model is intended to provide prediction regarding the range of possible values for the number of plasmid transfer per cell cycle and the cell cycle point when conjugative transfer are most likely to happen.

Sensing - All process defined over the agents implicitly sense the local environment and the close neighborhood for their decisions.

Interaction - Bacterial cells interact with their nearby individuals for nutrient access, cellular division, mate pair formation and plasmid transfer.

Stochasticity - Stochasticity is introduced at individual level for all cellular process 
sampling a normal deviate and fitting the value to corresponding process.

Collectives - No collectives are taken into account in this model.

Observation - All state variables will be saved at intervals of one minute of simulated time.

\subsubsection{Initialization}

The simulation model is initialized with a population of plasmid free $(R)$ and plasmid bearing $(D)$ cells according to input parameters. The agents are placed randomly within a circular surface centered over the lattice central position. The radius of circle where agents are placed is calculated as function of $N_{0}$ in order to be consistent to the desired initial cell density[ZZF+ ${ }^{+}$12]. The simulation environment is also initialized with a number of nutrient particles 5 in order to support the half of the estimated number of cellular divisions and the rationale behind it is to capture the intercellular competition for nutrient access.

The model input and initialization requires the parameters shown in Table 7.1. The costT4SS is the total cost of pili expression. The cost applied for a single pilus expression is costT4SS/param(maxpili). The param (maxpili) is currently a constant, having the value of 5 for the virtual E. coli cells simulated with BatoSIM.

The cellCycle parameter, in the BactoSIM version 1.0 indicates two things: the type of modeling rule and its parameter. A value of -1 set the model to conjugate as soon an infected cell finds a susceptible one. Setting the parameter to 0 will randomize de conjugation time between $t_{0}$ and $G$. Finally using a value greater than zero indicates the specific point in the cell cycle for conjugation. In subsequent versions, a new input parameter has been added to the BactoSIM specification for indicating the model type. Therefore, in versions greater than the 1.0 the semantics of the cyclePoint parameter, only indicates when conjugative activity is most likely to happen and the parameter only is used when the model type is equal to 3 . The model also needs an equation, fitted to the experimental data, where the dependent variable represents the conjugation rate $T /(T+R)$. Setting isConjugative flag to false creates a simulation where the transconjugant cells are unable to conjugate. The parameter equation is used for

\footnotetext{
${ }^{5}$ In the current version the number of nutrient particles is also an input parameter.
} 
assessing the quality of simulation output.

Table 7.1: The list of initialization parameters for BactoSIM 1.0 model.

\begin{tabular}{lll}
\hline Parameter & Unit & Description \\
\hline G & minutes & Average doubling time for plasmid free cells \\
cellCycle & $\%$ of $\mathrm{G}$ & The percentage of cellular cycle for conjugation \\
costConjugation & $\%$ of $\mathrm{G}$ & The penalization due to a conjugative event \\
costT4SS & $\%$ of $\mathrm{G}$ & The Pilus expression cost \\
$\gamma_{0}$ & c/cycle & Upper limit for conjugations performed by an \\
& & agent \\
isConjugative & true-false & Defines a conjugative or a mobilizable plasmid \\
isRepressed & true-false & The T4SS expression state for the plasmid \\
$N_{0}$ & cells/ml & Initial population expressed in cells $/ \mathrm{ml}$ \\
donorRatio & $\%$ of $N_{0}$ & The initial density of donor cells $(D)$ \\
Equation & $\mathrm{N} / \mathrm{A}$ & An equation for experimental data \\
\hline
\end{tabular}

The parameter Equation previously mentioned in the Table 7.1 is basically a third degree polynomial equation which has been fitted to the experimental data kindly provided by [ $\left.\mathrm{dCRC}^{+}{ }^{12}\right]$. This polynomial model has been generated using the standard $\mathrm{R}$ functions $\mathrm{lm}$ and poly. The reason for using an equation is due to practical reasons for making easier the visual assessment between the simulated and the experimental data, as well as, for defining the objective function required for the application of evolutionary methods to the model parameter estimation problem. The equations are shown in the Table 7.2 which contains the fitted models for the plasmids $p A R 118, p A R 115$, $p S U 2007$ and $p R 388$ (OriT) for two experimental setups with varying the initial donor densities 5 and $50 \%$. The data is representative of repressed, de-repressed and of non-conjugative/mobilizable plasmid. 
Table 7.2: The polynomial equations fitted to the experimental data of an experimental setup with conjugative four conjugative plasmids with different intial densities.

\begin{tabular}{lrc}
\hline Plasmid & D (\%) & Polynomial equation \\
\hline pR388(OriT) & 5 & $y=0.0017-2.5 e^{-4} \times x+4.6 e^{-6} \times x^{2}-6.8 e^{-9} \times x^{3}$ \\
pR388(OriT) & 50 & $y=0.0056+1.9 e^{-3} \times x-1.7 e^{-8} \times x^{2}-2.0 e^{-9} \times x^{3}$ \\
pSU2007(R388) & 5 & $y=0.038-2.1 e^{-3} \times x+1.7 e^{-5} \times x^{2}-1.9 e^{-8} \times x^{3}$ \\
pSU2007(R388) & 50 & $y=0.02-5.1 e^{-4} \times x+1.5 e^{-5} \times x^{2}-2.3 e^{-8} \times x^{3}$ \\
pAR115 & 5 & $y=-0.046+1.6 e^{-3} \times x+1.1 e^{-5} \times x^{2}-2.0 e^{-8} \times x^{3}$ \\
pAR115 & 50 & $y=-0.13+1.1 e^{-2} \times x-3.4 e^{-5} \times x^{2}+3.5 e^{-8} \times x^{3}$ \\
pAR118(R1) & 5 & $y=0.012-2.0 e^{-4} \times x+1.6 e^{-6} \times x^{2}-2.2 e^{-9} \times x^{3}$ \\
pAR118(R1) & 50 & $y=0.065-3.5 e^{-4} \times x+4.1 e^{-6} \times x^{2}-6.0 e^{-9} \times x^{3}$ \\
\hline
\end{tabular}

\subsubsection{Sub-models}

Nutrient Diffusion - The diffusion process uses the same approach described in $\left[\mathrm{KLF}^{+} 07\right]$ which is quite simple but effective capturing the essence and effects of diffusion process. Roughly speaking, the technique allows nutrients to be consumed not just from local lattice cell but also from its neighborhood. Thus, a size 3 Moore's neighborhood is used (a block of $7 \times 7$ lattice cells) for nutrient uptake but always giving priority to the locations closer to the cell. Once the corresponding nutrient uptake is calculated, then the model logic try to retrieve such quantity from the local lattice site if possible. If there are no nutrient particles available at the current cell site we select a random place at a neighborhood distance equal to 1, if the nutrient concentration at this site is greater than zero perform uptake from site otherwise remove it from list and try again the same process at lattice distances of 2 and 3 . When no nutrient particles are found, return to some randomly selected lattice site at distance equal to one and repeat the process until find a site with a nutrient concentration greater than zero or when all sites were visited. Such search is symmetric and makes nutrients closer to the bacterial cell position, much more prone to be consumed which in some extent emulates the real flow of nutrient particles towards clusters of bacterial cells due to resource depletion in such areas $\left[\mathrm{KLF}^{+} 07\right]$. 
Nutrient Uptake - The uptake process always tries to consume one nutrient particle, assuming a simple functional dependence between the growth rate $\mu$ and the rate limiting substrate availability as can be seen in (7.2), that is to say when no nutrients are available the growth rate is zero and when substrate $S$ is greater than zero, the growth rate will be the maximum growth rate, as defined indirectly in the doubling time (g) parameter.

$$
\mu(S)=\left\{\begin{array}{l}
S=0, \mu(S)=0 \\
S>0, \mu(S)=\mu_{\max }
\end{array}\right.
$$

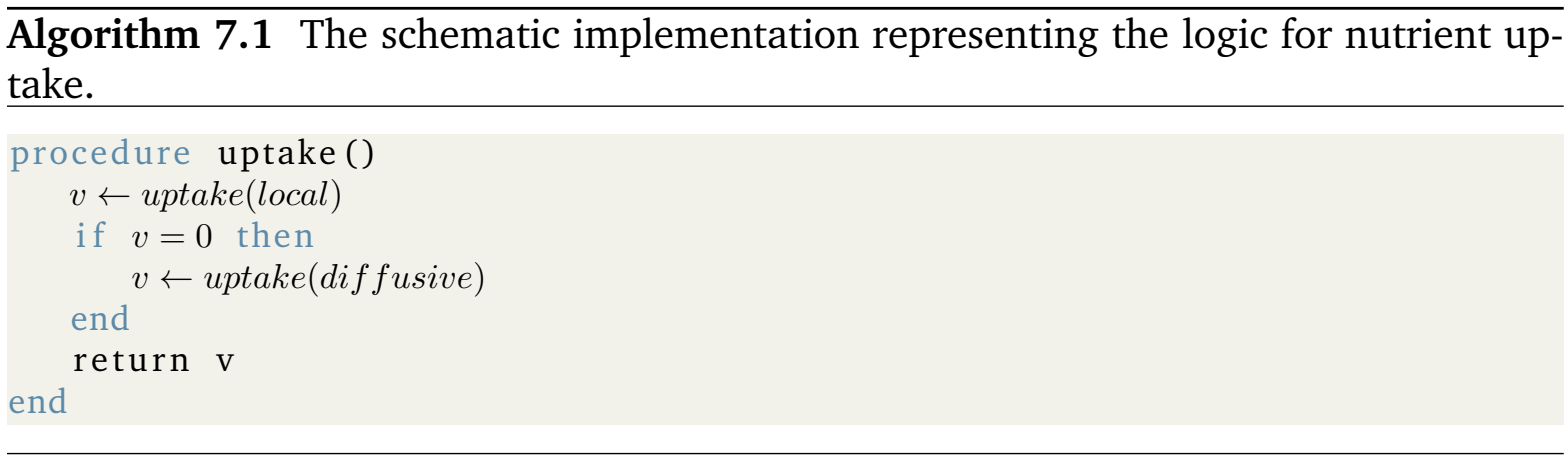

The complete nutrient uptake process is presented in 7.1 which basically shows that the local nutrient consumption will be tried first and in the case that local site contains no nutrient particles the aforementioned nutrient diffusion process is used.

Cell division - For each time step of simulation process a normally distributed random variable $Z_{g}$ with a mean equal to the user provided parameter generation time (G) and standard deviation $\sigma_{g}$ will be generated as shown in (7.3)

$$
Z_{g}=\sigma_{g} Z+\bar{g}
$$

Where $Z_{g}$ is a random variable sampled from a standard normal distribution, the $\bar{g}(R)$ is the average doubling time for plasmid free cells. The generated value $Z_{g}$ is then compared to the actual cell time defined as $\Delta_{t}=t-t_{0}$ being $t$ the current simulation time tick and $t_{0}$ the time of last cellular division. The standard deviation is assumed from an coefficient of variation of $10 \%$ which is the common values for a exponentially 
growth cultures.

Algorithm 7.2 The schematic implementation of time-based logic for bacterial cell division.

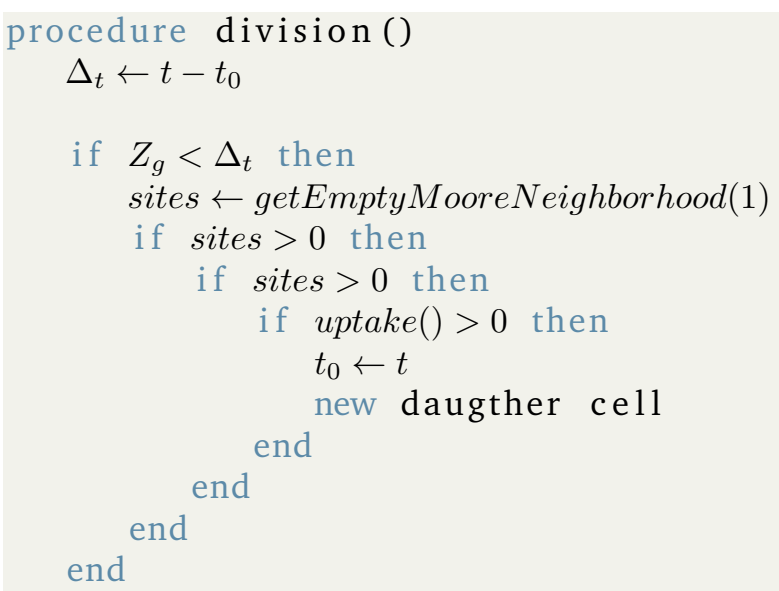

Thereby, the effective division rule can be seen in Algorithm 7.2. First the current value of $\Delta_{t}$ is compared to $Z_{g}$ and, if it is greater than or equal to the estimated doubling time $g$ for the current agent the process continues and the availability of empty sites is checked. Subsequently the agent will try to consume one particle of nutrient, first from current grid cell and, if there is no available nutrient in the local lattice site, the agent will try to consume the required nutrient particle using the diffusive process as described previously. Finally, if all conditions are met, a new daughter cell with the same conjugative state of the parent cell will be placed randomly at one of available empty sites.

Conjugative transfer - We have modeled the conjugation process using three different approaches which we have called strategies or model types. The distinctive point between these alternative implementations is the decision criteria for the time when the conjugative event will be signaled, that is two say, when a donor or transconjugant cell, that have already found a plasmid free recipient, will try to infect this recipient. The complete algorithms for the strategies 1, 2 and 3 are shown in Algorithm 7.3, 7.4 and 7.5 respectively. 
Thus, the first strategy, described by the Algorithm 7.3, does not take time into account and conjugation will take place simply when any recipient cell is found on the donor neighborhood.

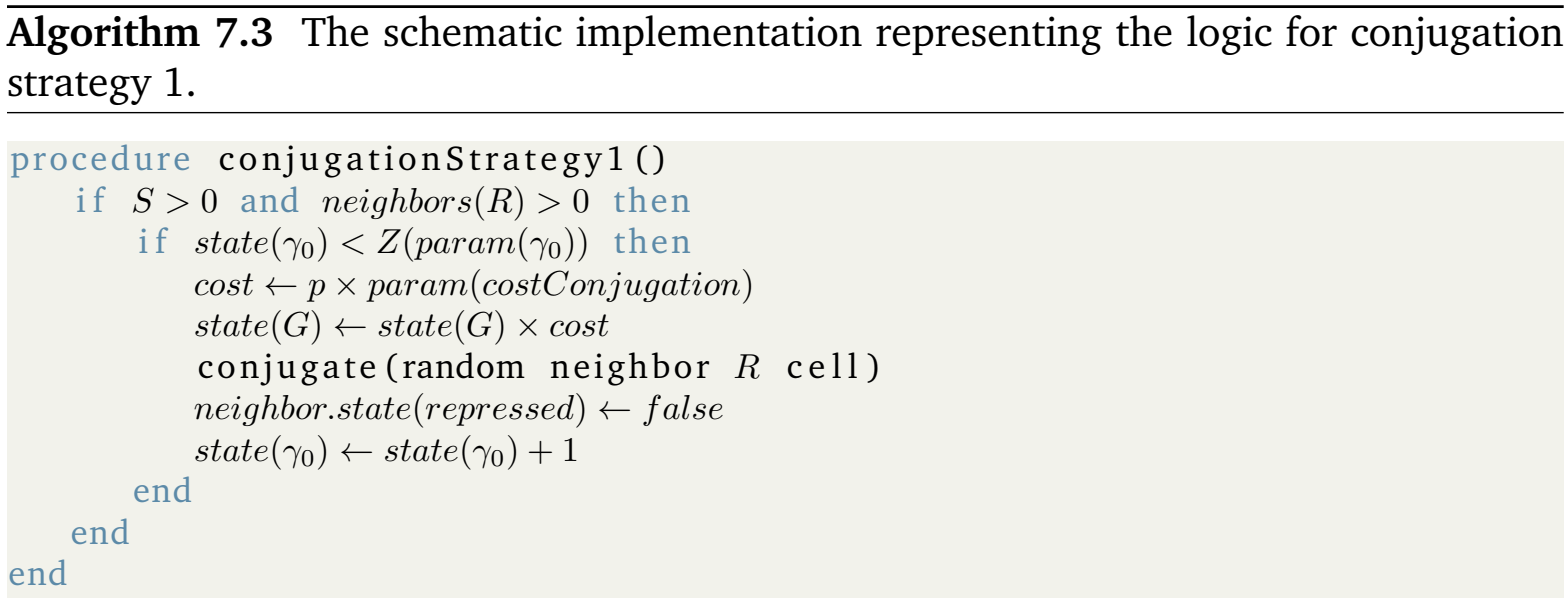

The second strategy, shown in Algorithm 7.4, uses a random number drawn from a uniform deviate being the number in a range of 1 and the cell doubling time, thus on average, conjugation will must prone to happen at a $50 \%$ of cell cycle but with a high standard deviation which is given by the expression $G-1 / 3.464102$.

Finally, in the strategy 3, depicted in Algorithm 7.5, a more specific time selection mechanism is used being the uniform random variable replaced by a normally distributed one with a small coefficient as low as a ten percent which is approximately three times lower than the case of strategy 2.

In all strategies the actual value of local $\operatorname{state}\left(\gamma_{0}\right)$ is compared to a normally distributed random variable $Z\left(\gamma_{0}\right)$ generated using the model parameter $\operatorname{param}\left(\gamma_{0}\right)$ and assuming again a coefficient of variation equals to $0.1\left(Z\left(\gamma_{0}\right)=0.1 \gamma_{0} Z+\gamma_{0}\right)$. The $\operatorname{state}\left(\gamma_{0}\right)$ is just the number of conjugations which have already been performed by the agent. All strategies also check for the nutrient availability $(S)$ and the presence of infectable $R$ cells on the agent neighborhood. The parameter $\gamma_{0}$ can be less than the unit and in this case it is indicating the relative frequency of agents which undertake at least a conjugation round. Thus, the parameter is used for deciding if an agent 

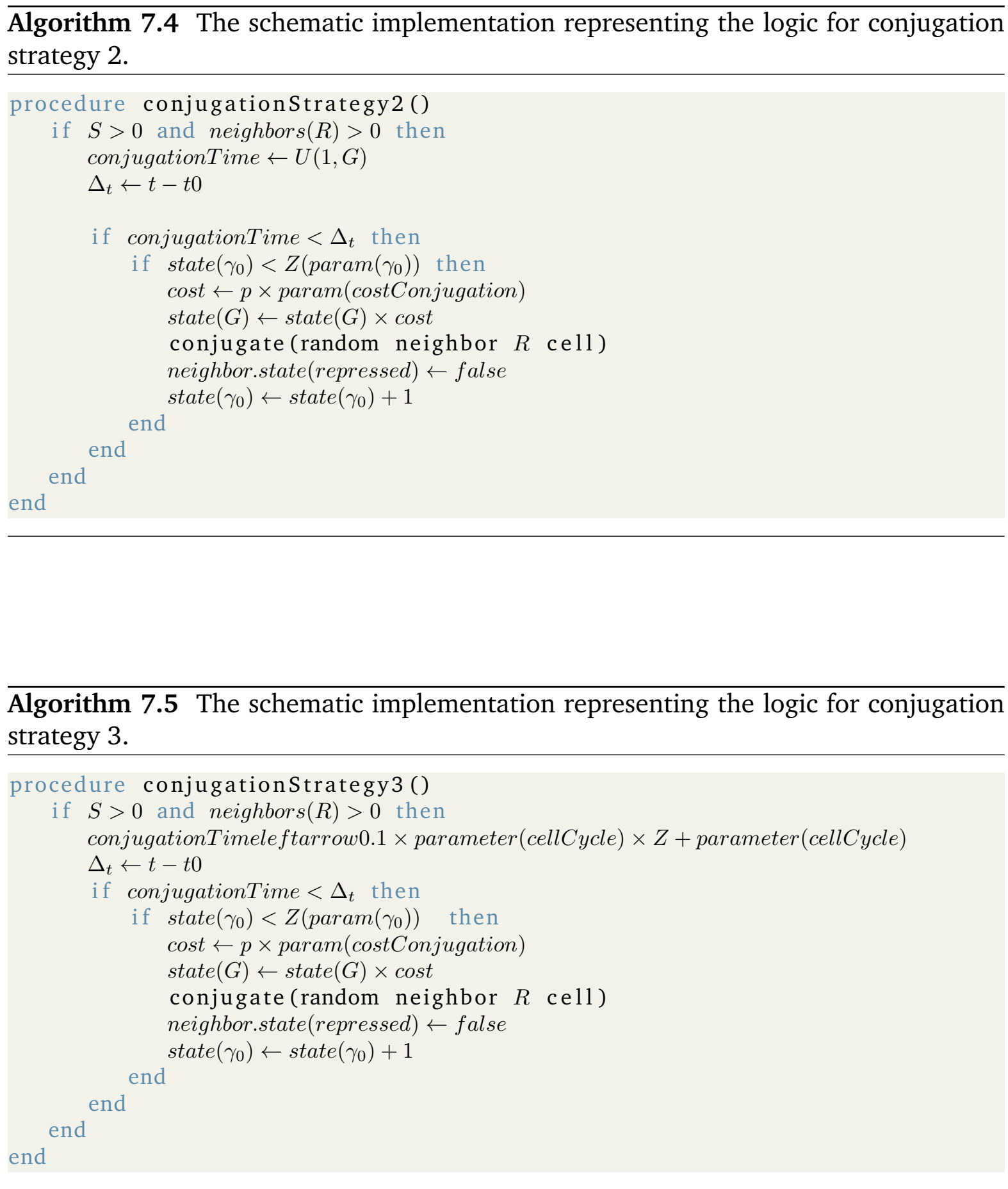
must conjugate, comparing a uniformly distributed random variable with the value of $\gamma_{0}$ divided by the agent doubling time.

Shoving relaxation - In order to capture the effect of bacterial cells pushing each during the colony growth and expansion we had implemented a simple version of the shoving relaxation process. We are interested mainly on emulating the net effect of colony expansion in the modification of the local neighborhood structure besides of building a relatively realistic colony expansion pattern. Our implementation assumes that bacterial cells are simple circular entities with a radius $r$. Although we are using a discrete grid with every agent occupying exclusively just one of the grid cells, the bacterial agents are allowed to have radius with values greater than actual grid cell which actually is a square of $1 \times 1 \mu \mathrm{m}$.

Thus, the cell radius $r$ is estimated using a simple linear relation between the current cell cycle point and the cellular elongation as shown in equation (7.4).

$$
r=s_{0}+\left(s_{g}-s_{0}\right) \frac{\Delta_{t}}{Z_{g}}
$$

Where $s_{0}$ and $s_{g}$ are respectively the half of minimum cell size and the cell size at division, which are assumed to fall between 0.5 and $3.0 \mu \mathrm{m}$ for rod shaped cells.

The shoving vector for every bacterial cell which overlaps with a neighbor is calculated in the same way as described in [KBW98]. For every bacterial cell present in the computational domain we calculate a so called shoving vector using a Moore's neighborhood size 1 , that is to say, accounting for the eight closest neighbors. The shoving vector $\vec{s}$ is calculated as using the equation (7.5).

$$
\vec{s}=\sum_{i=0}^{n} \frac{k r+r_{i}-\left\|\overline{P N_{i}}\right\|_{\hat{u}_{i}} .}{2}
$$

Where $\vec{s}$ is the shoving vector, $k$ is a constant used to set the maximum allowed distance or overlap between two adjacent cells, $r$ is the radius of current bacterial agent, $r_{i}$ is the radius of neighbor $i, \overline{P N_{i}}$ is the vector defined by the point centered at current cell and their neighbor $i$ and finally $\hat{u}_{i}$ is the unit vector from the center of neighbor $i$ to the current cell. The bacterial cell position is then moved according to the vector $\vec{s}$. The rationale is to model the effect of passive movement of cells in the modification of the neighborhood structure which may facilitate plasmid donor cells to 
reach more individuals susceptible to be infected.

T4SS expression - This rule models the expression of conjugative pili which required for conjugative events take place. The Type IV secretion systems (T4SS) is a sort of transmembrane protein responsible, amongst other things, for the mating pair stabilization and the injection of single strand DNA into the target recipient cell. On E. coli hosts, the average number of conjugative pili over cell surface is rounding five. The T4SS is an indispensable part of conjugative plasmid and, depending on the plasmid type, can be found constitutively expressed or repressed. The plasmids that have constitutive expression are continuously keeping the level of expression constant and as consequence the number of pili over cell surface is also stable. On the other hand, repressed plasmids, once the negative feedback control system has been established, do not enforce the expression of conjugative pili and the direct effect is that plasmids become unable to perform conjugations. Nonetheless, bacterial cells which have been recently infected present what is known as transitory de-repression allowing the transconjugant cells express the conjugative machinery and conjugate at higher frequencies.

We have implemented an straightforward and simplified version of the real process but capturing yet the most significant aspects of the pili expression subsystem, namely the intra and inter-cellular competition. The intra-cellular competition is modeled as a cost in the cell doubling time applied every time the cells require the expression of conjugative apparatus. The inter-cellular competition is achieved by requiring the uptake of a nutrient particle for the expression of pili. The logic of T4SS expression is shown in the Algorithm 7.6. Initially, in lines 1-2, we check whether plasmid is repressed and if the current expression level is above the desired level, in both cases no further actions are taken and we simply exit the process. When the plasmid is not repressed and the number of conjugative pili is under the average maximum number of pili, we start the expression logic but first trying to uptake a nutrient particle, as can be observed in line 4, in order to capture the inter-cellular competition.

The next step is to estimate the number of pili which will be generated and this is carried out simply by sampling a random deviate with uniform distribution between 1 and the parameter representing the average number of maximum number of pili present in cellular surface. The next step is to estimate the total cost to be applied and this is carried out multiplying the pili number by the unity cost of pili expression 
as is shown in line 6. In the next steps the current cell generation time is increased proportionally to the estimated cost. Finally the cell is returned to repressed state if the plasmid is a repressed one in line 10 and that is, transitory de-repression logic. It must be reminded that during conjugation we set the state(repressed) to false in the new transconjugant cell.

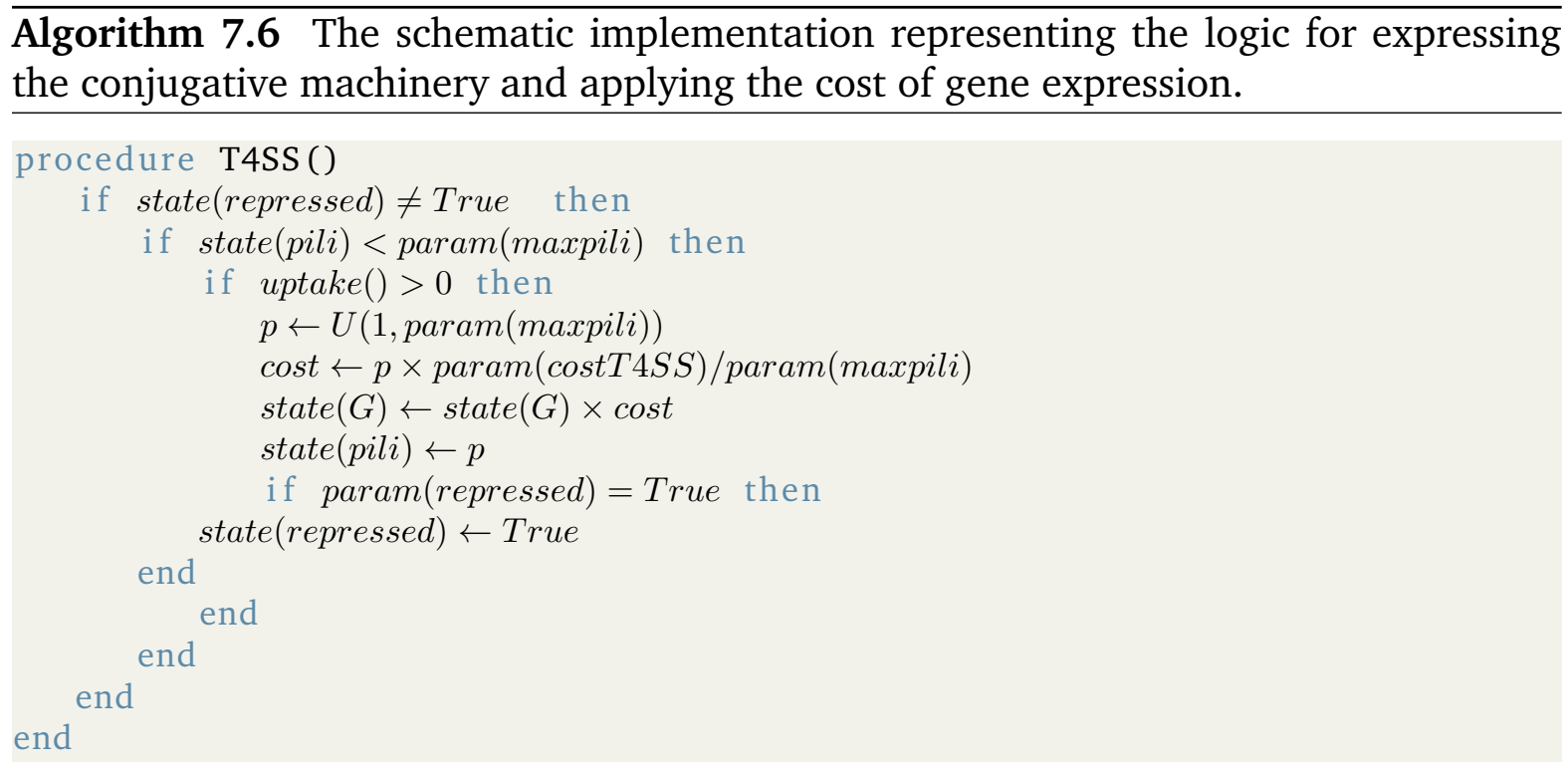

\subsection{Results and discussion}

Using the model described in the previous section, we have simulated and informally assessed two different plasmids, namely the pSU2007, which is based on the R388 backbone and the pAR118 which is a R1 derivative plasmid. The first plasmid is constitutively expressing the conjugative T4SS apparatus whereas the second is a naturally repressed and new formed transconjugant cells undertake the transitory de-repression and repression cycles. The experimental values of conjugation rates, which has been used for assessing the model quality, serving as reference data, has been kindly provided by the Intergenomic $\mathrm{Lab}\left[\mathrm{dCRC}^{+} 12\right]$. The parameters used in the simulation rounds are shown in Table 7.3. It is worth to note that these values are simple approximations obtained using some assumptions about the conjugation and a trial and error process. 
Table 7.3: The initialization parameters for conjugation experiments. The first column is the name of real plasmid being simulated and the number inside parentheses indicates what strategy is being used. The cost $_{1}$ and cost $_{2}$ are respectively the cost of conjugation and the cost required to express the T4SS conjugative machinery.

\begin{tabular}{lrrrrrrrl}
\hline Name & G & cellCycle & cost $_{1}$ & cost $_{2}$ & $\gamma_{0}$ & $N_{0}$ & $\mathrm{D}(\%)$ & repressed \\
\hline pSU2007(S1) & 43 & N/A (-1) & 5 & $60 \%$ & 3 & $9 \times 10^{9}$ & $50 \%$ & false \\
pSU2007(S2) & 43 & N/A (0) & 5 & $60 \%$ & 3 & $9 \times 10^{9}$ & $50 \%$ & false \\
pSU2007(S3) & 43 & $75 \%$ & 5 & $60 \%$ & 3 & $9 \times 10^{9}$ & $50 \%$ & false \\
R1(S1) & 43 & N/A (-1) & 5 & $40 \%$ & 2 & $9 \times 10^{9}$ & $50 \%$ & true \\
R1(S2) & 43 & N/A (0) & 5 & $40 \%$ & 2 & $9 \times 10^{9}$ & $50 \%$ & true \\
R1(S3) & 43 & $75 \%$ & 5 & $40 \%$ & 2 & $9 \times 10^{9}$ & $50 \%$ & true \\
\hline
\end{tabular}

As can be observed by the simple visual assessment of the Figure 7.4, under the same initial conditions, the strategy 1 and the strategy 2 clearly overshoots the experimental curve significantly, both in slope as in the final values of conjugation rates. In the case of repressed plasmid pAR118, the first two strategies do not provide a good adjustment to the experimental data. On the other hand, the strategy three seems to provide naturally a good fit to the real data, being both curves pretty close and with similar shapes. The strategy 1 is apparently the worst for implementing the local conjugation rule for agent-based models.

The second strategy, although the conjugations events occur on average at a $50 \%$ of cell cycle is also very far from the experimental data. That seems to indicate that there are some timely mechanisms which control the plasmid infection wave in a bacterial colony. The exact underlying mechanism is not yet known but, it seems to exist some weak evidence on that point, that is to say, if conjugation is allowed to happen two early in the cellular cycle the plasmid is flooded to the whole colony at a much higher speed that would be expected. These points are linked to the research questions Q1 and Q2. 


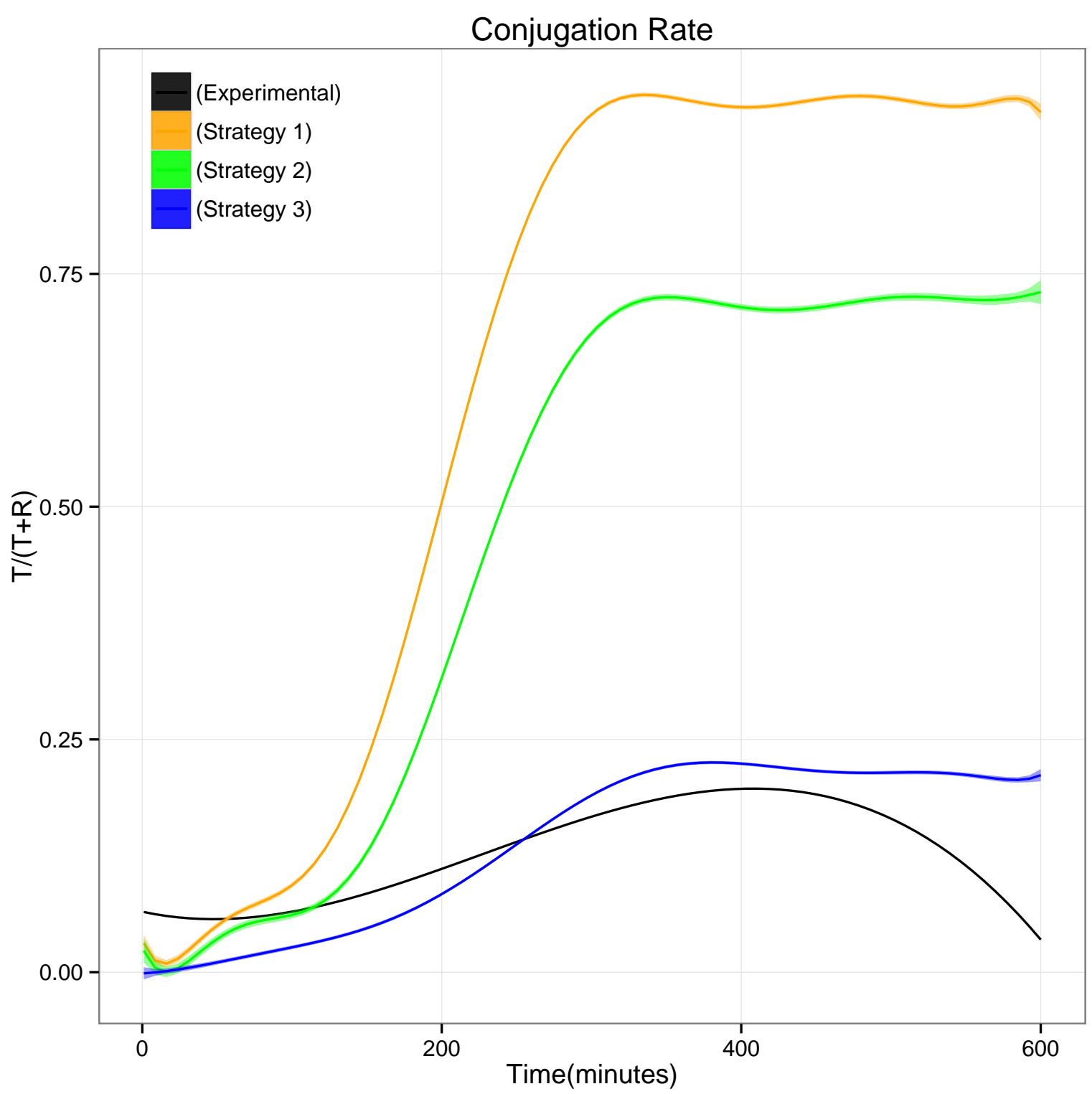

Figure 7.4: The simple visual assessment of three strategies for implementing the conjugation rule for the plasmid $p A R 118$ (R1) with an initial donor density of $50 \%$. The black line is the reference experimental data.

Similarly, the preliminary results for the de-repressed plasmid pSU2007 shown the Figure 7.5, are telling practically the same thing as in the previous case. The both strategy 1 and strategy 2 provides a very poor fitting, falling very far from the experimental curve. Again, the winner is the strategy 3 following the reference data very close and 
naturally.

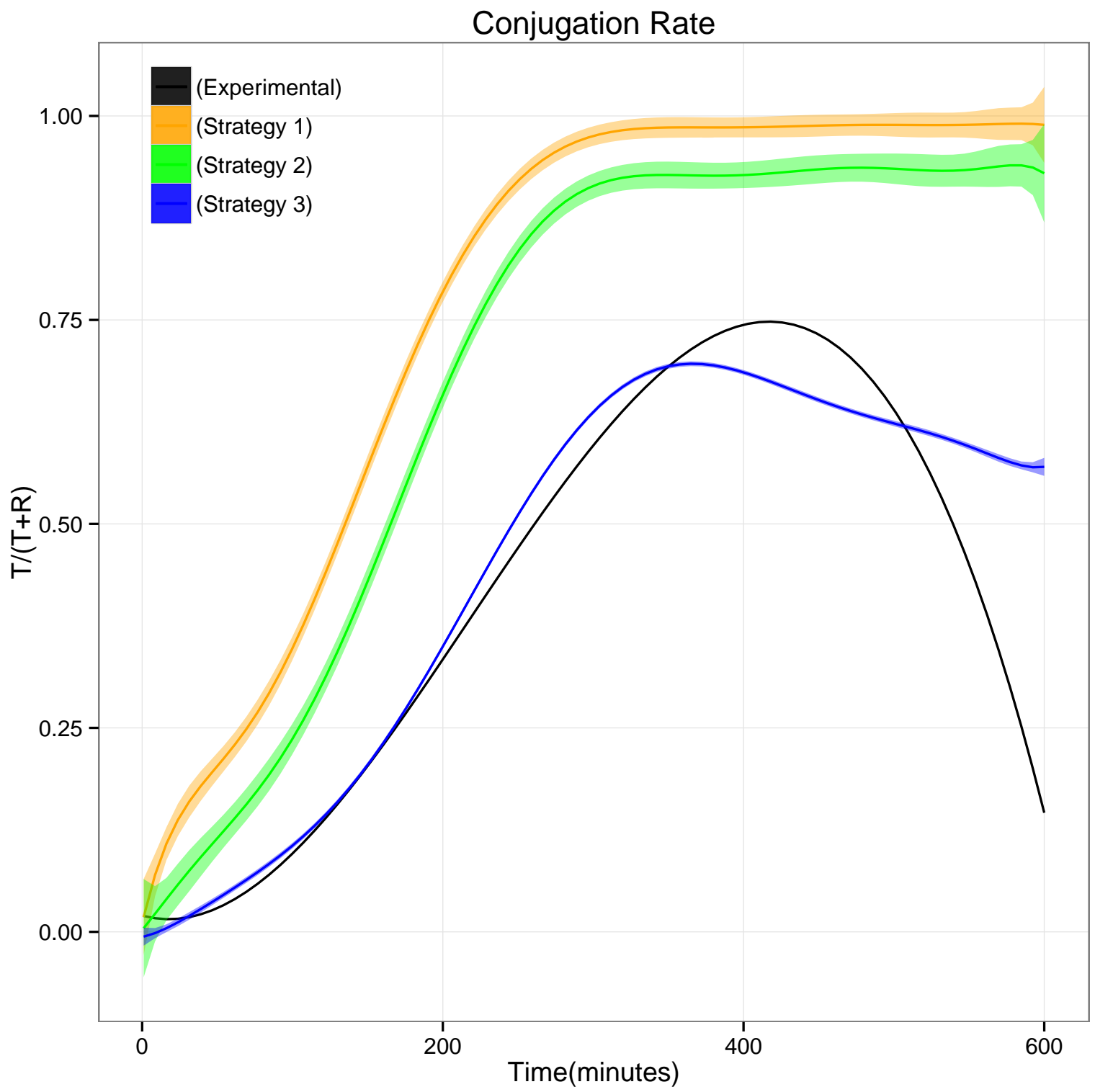

Figure 7.5: The simple visual assessment of three strategies for implementing the conjugation rule for the plasmid pSU2007 with an initial donor density of $\mathbf{5 0 \%}$. The black line is the reference experimental data.

Returning to the Figure 7.1, we can observe how the donors, transconjugants and uninfected cells gradually, along the colony growth, become physically separated from 


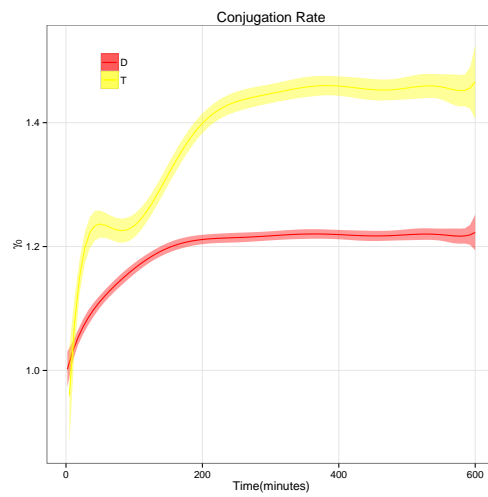

(a) Strategy 1

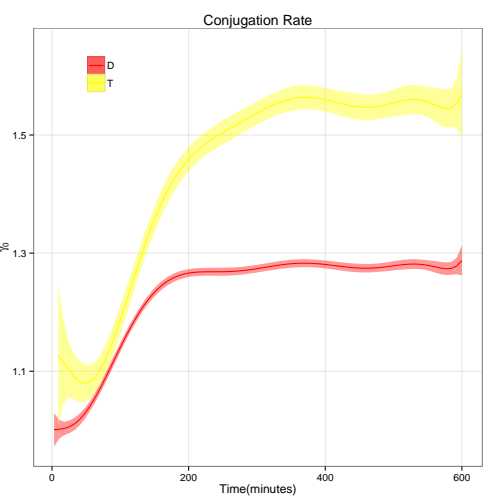

(b) Strategy 2

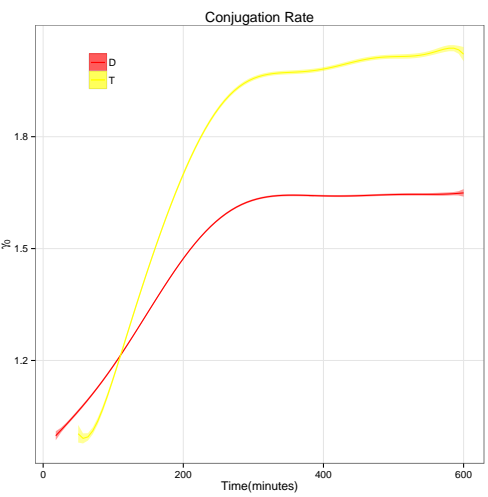

(c) Strategy 3

Figure 7.6: The average values of $\gamma_{0}$ output of donors (D) and transconjugant (T) cells generated using the BactoSIM model for comparing the effect of the three different strategies (model types). These are the results of simulating a derepressed plasmid (pSU2007) using an initial donor density equal to $50 \%$ of the total population. The color scheme used here is red and yellow respectively for donors and transconjugant cells.

each other (research questions Q3 and Q9), forming demes of similar individuals as the zones of active expansion move towards the edges. Hence, even when substrate is readily available, the conjugative active remains restricted to the small strip connecting both sub-population areas. This observed growth pattern seems to be in line with the results reporting that the genetic drift within the bacterial colony may stop the conjugative activity[[FKJC14].

The average output for the plasmid pSU2007, using an initial donor density of 50\% are shown in the Figures 7.6, 7.7 and 7.8 each of them presenting, respectively the metrics $\gamma_{0}, R_{0}$ and the percentage of total plasmid infections due to the horizontal transfer for the three strategies/model types addressed in this work. The input parameters used for running these simulations are those shown in table 7.3 for the plasmid pSU2007.

It is interesting to note, in the Figure 7.6, the apparently counter-intuitive effect of conjugation pace in the average values of $\gamma_{0}$. The first two strategies despite of the higher speed of infection wave have lower values of $\gamma_{0}$ than the strategy 3 . The cause of that apparent contradiction can be attributed to the fact that if cells are able to forward 
the plasmid earlier more cells are infected and able to infect soon which implies an average small number of conjugations performed on a cell basis.

Interestingly, the values of $\gamma_{0}$ estimated by all strategies are, on average, lower than the values provided as the model parameter (see the Table 7.3). Apparently, this is a consequence of physical separation between infected and uninfected cells which limits the number of infectible neighbors (research questions Q3 and Q9). Although, some of the simulated donors or transconjugants in the whole population can reach the upper limit, the most of individuals cannot because of other physical constraint, such as the neighborhood structure or nutrient availability.

The intrinsic conjugation rates of donor cells stabilizes sooner than the transconjugants which continues increasing reaching higher values of $\gamma_{0}$. This reflects the fact that once the donors cells initiates the process, rapidly become surrounded by transconjugant ones which become the dominant factor in the infection speed possibly due the barrier of formed. Additionally, the transconjugant cells are susceptible of overexpressing the conjugative genes owing to the transitory derepression[SGSL90][RWZ12][LL86][FLdCR $\left.{ }^{+} 14\right]$. But even without the transitory derepression the biophysical aspects would play an important role. In order to provide a more graphical example for this situation, let us define an optimal donor which is completely surrounded by uninfected cells. This optimal donor is so proficient that is able to completely infect all of it plasmid free neighbors and hence all of it become transconjugants very quickly. This leads to an apparent paradoxical situation because on average the transconjugant cells will have higher values of gamma $_{0}$ than the optimal donor.

The Figure 7.7 shows the metric $R_{0}$ which stands for the basic reproduction number or basic reproductive ratio for the simulated plasmid pSU2007 using an initial donor density of $50 \%$. This metric plays a central role in epidemiology and could be approximately defined as the mean number of secondary infections produced by an infected individual[BC93][KG00]. It serves as a reference for analyzing whether an infection will dominate of fadeout in some population. Usually, in most infections, the metric refers exclusively to the horizontal process alone, but in the case of the host-plasmid associa- 


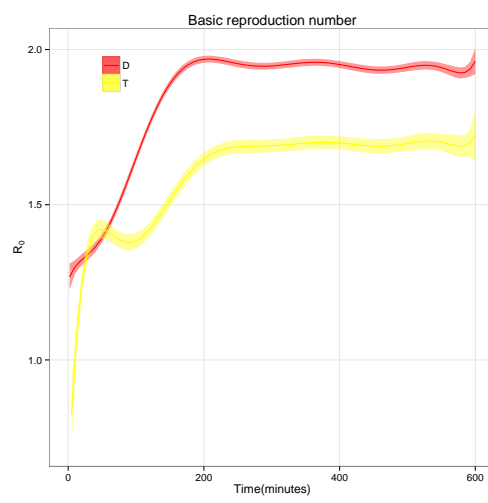

(a) Strategy 1

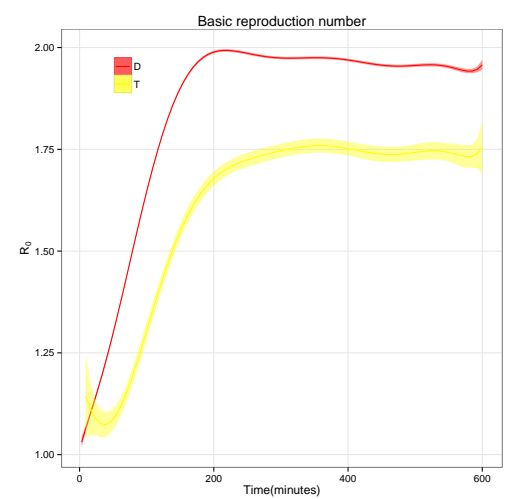

(b) Strategy 2

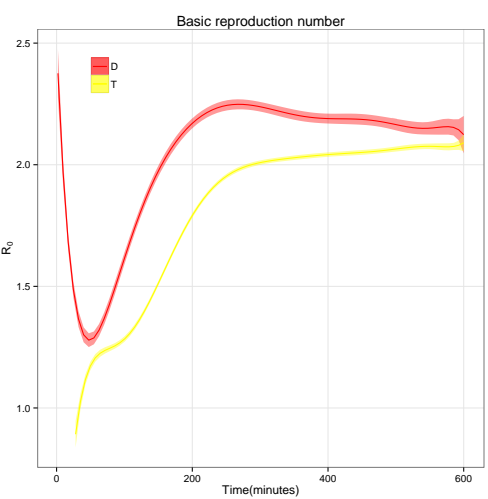

(c) Strategy 3

Figure 7.7: The average values of $R_{0}$ output of donors (D) and transconjugant (T) cells generated using the BactoSIM model for comparing the effect of the three different strategies (model types). These are the results of simulating a derepressed plasmid (pSU2007) using an initial donor density equal to $50 \%$ of the total population. The color scheme used here is red and yellow respectively for donors and transconjugant cells.

tion $R_{0}$ is the sum of both vertical and horizontal components [LCWF05] [LNEM95]. As can be observed, the pattern are the same for the three strategies/model types with the values of $R_{0}$ being greater for donors than for the transconjugants cells and increasing until become stable when reaching the 200 minutes of simulation.

The main idea which can be figured out from the values of $R_{0}$ metric (research questions Q3, Q4, Q5, Q6, Q7 and Q8) shown in the Figure 7.7 is about how the different epidemic strategies 6 are affecting the evolution and the maintenance of the plasmid in the population. The values for the basic reproductive number of the donor sub-population clearly outcompete the transconjugants cells, showing a more effective plasmid persistency strategy. The focal point here is that the donor cells (D) have to invest less metabolic resources in the horizontal transfer which could be attributed to the plasmid-host coadaptation and possibly to the fact that the donor cells already have expressed to an stable level, most of the genes associated to the conjugation. Consequently, donor cells are able to reproduce faster than the transconjugants, which increments the vertical component of $R_{0}$ making them more efficient. On the other

\footnotetext{
${ }^{6}$ Not to be confounded with the modeling strategies
} 


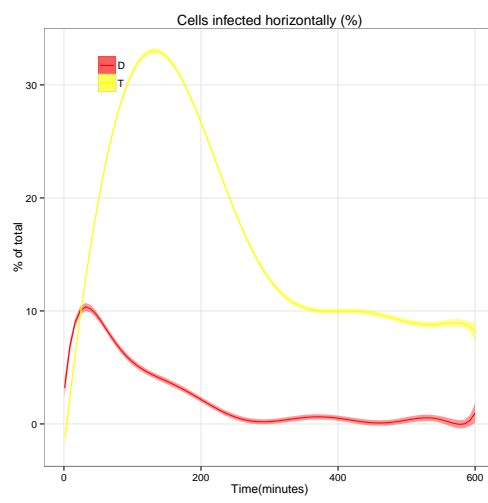

(a) Strategy 1

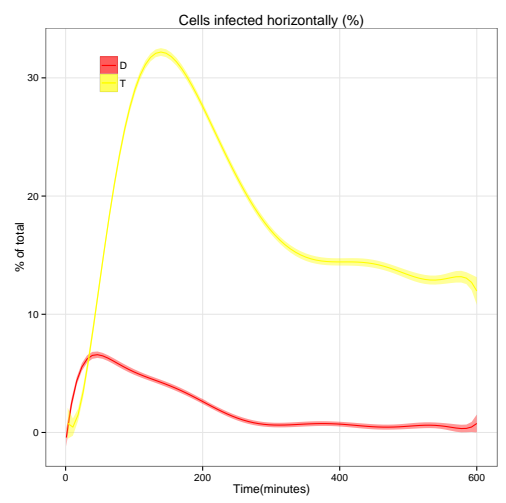

(b) Strategy 2

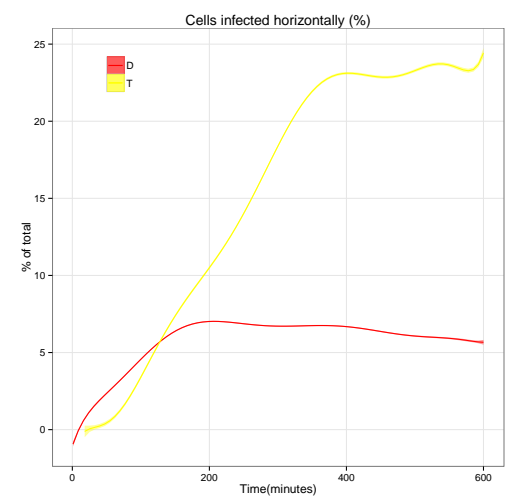

(c) Strategy 3

Figure 7.8: The average values of horizontal spread output of donors (D) and transconjugant $(\mathrm{T})$ cells generated using the BactoSIM model for comparing the effect of the three different strategies (model types). These are the results of simulating a de-repressed plasmid (pSU2007) using an initial donor density equal to $50 \%$ of the total population. The color scheme used here is red and yellow respectively for donors and transconjugant cells.

hand, the transconjugants have to dedicate a lot of resources for becoming proficient donors making them globally less efficient than donor cells. Hence, it can be assumed safely that if the metabolic penalizations are separated in a system of two components, one related to the cost of conjugation $C_{1}$ and another to the cost of expressing the conjugative apparatus $C_{2}$, then $C_{2}>C_{1}$.

The last metric shown in the Figure 7.8 is the relative importance which the horizontal component in the newly infected individuals for the three modeling strategies analyzed. The strategy 1 and the strategy 2 have both a similar pattern which indicates that the donor cells are only engaging in horizontal plasmid transmissions during the start of the simulation process. This certainly is no realistic and is possibly a consequence of the donor cells are conjugating two early forcing the reachable recipients to become quickly depleted (research questions Q1 and Q2). The strategy 3 again seems to produce results which are close to the expected behavior. As can be observed, the donor cells are keeping the average horizontal component under a very low threshold, approximately a $7 \%$ of total infections are due to the conjugation. The 
remaining infections are vertically transmitted to the offspring generated by the donor cells.

On the other hand, the horizontal contribution of transconjugant cells are significantly higher with an average value of approximately $25 \%$ of newly infected cells attributed to the conjugative transfer. The value of this metric is incrementing linearly until the simulated time is equal to 400 minutes. This is basically telling us that the infection strategy of transconjugant cells are clearly favoring the conjugation despite of the metabolic effort required for expressing the conjugative machinery and for the conjugative transfer itself. It would be much more reasonable, that donors choose that strategy as they have an inferior overall cost. Apparently, it is an evolutionary nonsense but with a possible underneath justification which is telling us that even at a micro-scale the spatial separation may play a significant role delimiting the adaptive landscape.

\subsection{Summary}

In this chapter we have introduced our modeling approach which has been called BactoSIM. The model structure has been thoroughly described using the ODD protocol and the output metrics generated by the model have been critically discussed. The model has been assessed informally and the results have allowed us to better understand the individual-level components which are shaping the emergent dynamics. It is important to make clear the terms used here for making reference to the alternative implementations of the conjugation rule, as they will be analyzed in the next chapter. We have defined three different implementations, differing with respect to the time in the cell cycle when the conjugative transfer takes place. These alternative implementations have been called strategies. In order to select one of these strategies the model implementation has an input parameter denominated model type which may assume the values 1,2 or 3 (models M1, M2 or M3). Hence, M1 is equal to strategy 1, M2 is equal to strategy 2 and $\mathrm{M} 3$ is equal to strategy 3 . The cyclePoint parameter is used only in the M3/strategy 3 and on the other strategies it has no effect as have been described previously in this chapter.

Finally, it is also worth to note that the values of parameters and outputs itself are not really important since there are a lot of uncertainty in these kind of models and all values should be taken as approximates. But what must be noted are the relationships 
between them and their relative magnitudes. The parameters for which experimental data are available must fall in realistic ranges and in their relative magnitudes. For instance, it could not be stated that the costs of T4SS expression and conjugation are those used in our simulations but it can be assumed safely that if some parameter is greater than other in the model the same property possibly holds in the real system. 


\section{Chapter}

\section{Analysis and discusion of the model output}

8.1 Overview $\ldots \ldots \ldots \ldots$. . . . . . . . . . . . . . . . . . . . 182

8.2 Definitions . . . . . . . . . . . . . . . . . . . . . . 183

8.3 The effect of temporal structure $\ldots \ldots \ldots$. . . . . . . . . . . . 184

8.4 The effect of cell density on initial contact delay . . . . . . . . . . . . 191

8.5 Description of model analysis . . . . . . . . . . . . . . . . . 194

8.6 Preliminary screening of model output $\ldots . \ldots 196$

8.7 Assessing the fitness of different models . . . . . . . . . . . . . . 208

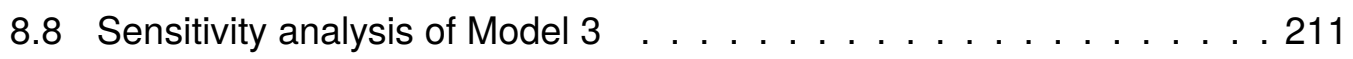

8.8.1 Sobol indices for a repressed plasmid . . . . . . . . . . 213

8.8 .2 Sobol indices for a de-repressed plasmid . . . . . . . . . 217

8.8 .3 Sobol indices for a mobilizable plasmid . . . . . . . . . . . 223

8.9 Summary . . . . . . . . . . . . . . . . . . . . . 238 


\subsection{Overview}

The primary purpose of this thesis is to provide a robust operational model for conjugation using an individual-based approach which can be easily adapted and used a standard modeling tool for simulating the kinetics of conjugative plasmids. In order to accomplish that goal, we must bring to the light some hidden aspects of conjugation which cannot be observed in whole population experimental setups and only can be understood using a bottom-up modelling. That approach has an added value because at the same time that we produce a more dependable model, we are generating knowledge and trying new hypothesis about the of the local structure, as well as, the intra-intracellular and the individual-level factors responsible for the global dynamics.

That is not an easy task because it is necessary to make many assumptions and simplifications to provide a usable abstraction for the process. It has been used in other works as the operational abstraction for bacterial conjugation, a set of rules relying on parameters like some arbitrary probability value, the pilus scan speed, the action radius of conjugative pili [MLS ${ }^{+11]}$ or even simply the number of infected individuals on the neighborhood [KLF+ 07$]$.

As a general rule, the good initial assumptions for individual-based models are those which are biologically consistent and could be almost axiomatically accepted. The assumptions, which fall in this category, are fundamentally that most of the processes inside of any cell are uphill which basically means that they have fight against an energy gradient or in other words, that they have a cost. The second assumption, is that cellular processes are subjected to a precise set of timing constraints for all cellular activities and the deviation on these timers is normally disruptive for cellular activities (temporal structure).

In order to thoroughly understand how the plasmids are distributed and evolve in a bacterial population it is necessary to separately identify the different aspects that affect the progression of cell to cell transmission and the invasion in the whole population. The final outcome of the process leading to the partial or total infection a bacterial colony, can be seen as a non-linear sum of a set of contributions due to vertical and ho- 
rizontal transfers as well as how much the metabolic burden contributes, as a negative feedback loop, to the pace of conjugative process.

In this chapter, we will analyze the individual-based model for bacterial conjugation, which has been described in the previous chapter. This model was implemented using an ensemble of component process which has been used to evaluate the better alternative for modeling and understand the conjugation systemically. Thereby, using this modular design, we have plugged different approaches (strategies/model types) for representing the conjugation with respect to the cell cycle in order to verify what is the best approach producing a natural fit to a complete and diverse experimental data. These alternative modeling approaches have been informally assessed in the previous chapter.

\subsection{Definitions}

In this section, we will provide some more stricter definitions for some terms we have used in our conjugation model. This terms are the intrinsic conjugation rate, the cycle point and the degrees of freedom.

\section{Definition of $\gamma_{0}$}

The term $\gamma_{0}$ is the total number of conjugative transfers carried out by a single bacterial cell between the period of two successive divisions. It is a metric representing partially the efficiency of an individual cell for infect its reachable susceptible neighbors. Actually, it has two different interpretations depending on its value. The first possibility is that $\gamma_{0}<1$ in this case it is as a probability in a binomial experiment, signaling the fraction of cells which could try to conjugate. The cells which conjugate will only perform one conjugative transfer. On the other hand, when $\gamma_{0} \geq 1$ all proficient donors could, at least theoretically, try to conjugate a number of times equal to the value of $g_{a m m a}$. The actual number of successful conjugations depends also on the availability of infectible recipients and on the cell cycle. 


\section{Definition of $\gamma_{1}$}

The term $\gamma_{1}$ represents the fraction of bacterial cell cycle when the conjugative transfer is most likely to happen. It must be a value from 0 to 1 , being 0 the birth of a new cell and 1 the time where the divides generating a new daughter cell.

\section{Degrees of freedom}

The total degrees of freedom in the number of model parameters minus the number of constraints defined over the model paramters.

\subsection{The effect of temporal structure}

The purpose of this section is to expose the rationale behind the idea that the conjugation is most likely to happen later in the cell cycle. In order to accomplish this objective we have created a simple abstract representation for the process which eliminates all other factors. Take into account that wave speed of plasmid spread in a bacterial population constitutes a complex and non-linear process and, as such it is hard to grasp any intuitive ideas about the main factors controlling the global behavior of the system as a whole. Hence, for understanding the spatiotemporal behavior and visualize the processes some simplifications are required to be made. Hence we have used a network analogy, comparing bacterial cells to network nodes which store and forward messages, being the conjugative plasmids the message transmitted from cell to cell.

It is known that most cellular processes follows a tight time schedule and is natural to think that the bacterial conjugation must be subject to the same constraints. Nonetheless until now, no works have studied how conjugative events are distributed across the cell cycle duration. With respect to time and ignoring other constraints, such as the availability of reachable susceptible individuals, we may state the following biologically relevant hypotheses: 


\section{Hypotheses}

- The time of conjugative transfer event is completely independent of bacterial cell cycle and grown conditions being merely a function of plasmid endogenous factors.

- On the other hand, plasmids could behave as all other cellular processes being linked to the host metabolic level and growth rate.

The main implication of first hypothesis, as should became clear in the next paragraphs, is that the larger is the bacterial generation time more efficient becomes the conjugative process because the infection rate surpasses the host growth rate allowing the complete plasmid invasion.

In the second hypothesis, the efficiency of a conjugative plasmid depends on the host growth regime and also on the plasmid intrinsic factors. Hence the time where conjugation will occur can be expressed as a percentage of bacterial doubling time indicating the fact that conjugative activity depends and is coordinated by the host dynamics.

It has already been pointed out recently[MLS $\left.{ }^{+11}\right]$ that time-related parameters (and consequently the associated delays) are the most important parameters governing the globally observed dynamics of plasmid spread in bacterial populations. The authors have used the sensitivity analysis[ $\left[\mathrm{MLS}^{+} 11\right]$ for identifying the input factors which are causing the variability in the model output. In this work, we are also trying to show that the temporal structure of the process, that is to say, the elapsed interval from the time when a cell become infected until it is able to forward the plasmid in a secondary infection round, is an aspect to be take into account.

The bacterial cellular dynamics controlling the growth and division process has a theoretical framework which has been pointed out as the central dogma bacterial cell division cycle or simply of Cooper-Helmstetter model[ZWV11]. The model describes the bacterial cell process relating a set of events such as the chromosome replication and division to the changes in the cell mass.

According to the BactoSIM model description presented previously in Section 7.3 , 
we have three basic cases for the temporal properties of bacterial conjugation, taking into account the bacterial cell cycle. We have called this as strategies/model types and in the context of this work the term strategy means the algorithm itself and the term model is the input parameter of BactoSIM allowing the user to select an specific strategy. The meaning of the model types are enumerated again in the box presented below:

\section{Cell cycle effects}

- Model 1 (M1) - Conjugation is independent of host dynamics and conjugation may take place as soon a suitable recipient is found on the neighborhood of plasmid bearing cell.

- Model 2 (M2) - The frequency of conjugative events is evenly distributed across the cell cycle.

- Model 3 (M3) - Finally, conjugation is linked and coordinated to the host dynamics being the frequency of conjugative events concentrated in some specific point late in the cell cycle.

The time related parameters of a conjugation model can be grouped, for the sake of simplicity, as a plasmid infection forwarding delay which stands for the lag time required for some plasmid being forewarned to any adjacent cell. Thereby we can study the forwarding delay effect on the slope of flooding speed.

In the most elemental level, the rate at which the infection progresses, depends on how many times every single cell can spread the plasmid, which hereafter we call intrinsic conjugation rate, it also depends on how fast the transmissions from donor cells to recipient cells and further retransmission from transconjugants cells can be accomplished. Thus, the intrinsic conjugation rate, ignoring other aspects, depends almost exclusively on the bacterial growth regime and on the time required for a complete plasmid transfer. Just to put forward a simple example to clarify that idea: Using a generation time $\mathcal{G}=20^{\prime}$, the average transfer speed, which is approximately $45 \mathrm{~kb} / \mathrm{m}$ and the archetypical $\mathrm{F}$ plasmid which has a size of $100 \mathrm{~kb}$, the minimal amount of time required for conjugation to complete would be roughly two minutes and the maximum 
intrinsic transfer rate would be 10 conjugations per cell cycle.

Let us assume a simple and yet idealized bacterial population of $N$ individuals with a growth rate $\mu=0$. The population will be sessile, which implies that the topology of network will be static and individuals cells will be distributed side by side conforming an interaction structure which could be abstracted by a linear graph as can be seen in Fig. 8.1 .

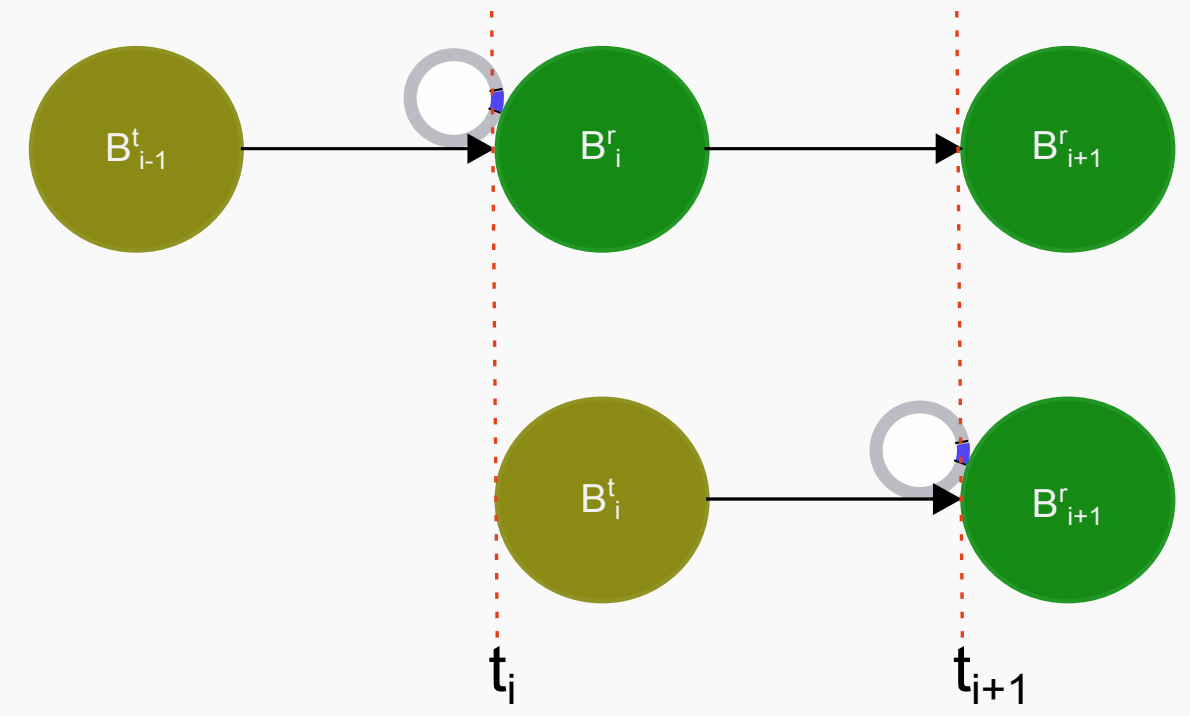

Figure 8.1: The scheme representing the imposed delay on bacterial networks. The $B^{r}$ and $B^{t}$ are respectively the recipient and transconjugant bacterial nodes. The figure shows the meaning of forwarding delay which is the time elapsed since a bacteria $B_{i}^{r}$ is infected becoming a transconjugant $B_{i}^{t}$ and infect the next recipient cell $B_{i+1}^{r}$ in the linear graph shown.

Hence assuming the functional similarity between conjugation and networks a common metrics can be used to analyze the process. One of these metrics is the total delay required for flooding all nodes in the network with some message $\mathcal{M}$ which in the bacterial context is given by the Equation (8.1)

$$
\tau=N \times T+(H-1) \times P+(H-1) \times T,
$$


being $\mathrm{N}$ the number of messages being forwarded, $\mathrm{T}$ the time required for transmitting the message, $\mathrm{P}$ the time needed for each node to process the message before being able to forward it again and finally $\mathrm{H}$ stands for the number of hops in or network. Putting it in terms of bacterial conjugation we have that $\mathrm{N}$ represents the plasmid size in kilobases, $T$ the time required to move a single kilobase from a cell to another, $\mathrm{P}$ may represent the two sources of delay which are referred as recovery and maturation time for donors and recipient cells respectively. Finally $\mathrm{H}$ is the actual number of bacterial cells in the linear topology already mentioned.

The aggregated value referred globally as maturation time includes, amongst other factors, (a) the time required for adding the second strand to the plasmid inside the new formed transconjugant cells, (b) the time required to express all genes coding the proteins for the trans-envelope apparatus, and (c) possibly the effect of SOS response triggered by entrance of single stranded DNA into the transconjugant cell[BBM10].

The Figure 8.2 shows the temporal evolution of the linear graph which denotes the bacterial population being infected. In this graph every donor cell, depicted by $B_{i}^{t}$ only have a reachable neighbor which can be infected in a conjugative event. In other words the plasmid bearing cell $B_{i}^{t}$ can only interact and conjugate with the bacterial agent $B_{i+1}^{r}$. Thus, in the simplest case where the forwarding delay is fixed, the infection speed can be approximated by a straight line in a form of $y=\alpha+\beta x$ being $\alpha$ the intercept and $\beta$ the slope. The value of slope for the most general case is given by the expression shown in Equation (8.2)

$$
\beta=\frac{\left|B^{t}\right| t_{i}-\left|B^{t}\right| t_{i-1}}{\Delta_{t}}, \forall i>0
$$

where $\left|B^{t}\right| t_{i}$ and $\left|B^{t}\right| t_{i-1}$ are respectively the counting of $B^{t}$ cells at $t_{i}$ and $t_{i-1}$ and $\Delta_{t}=t_{i}-t_{i-1}$. The intercept $\alpha$ is zero since at $t_{0}$ the number of $\left|B^{t}\right|$ cells are also zero. The choice of $\left|B^{t}\right|$ as the indicator of network flooding is just for the sake of generality but the same principles will also hold if instead of it the value of $T /(T+R)$ were used.

The Equation (8.2), in the case of unitary increments in the infected nodes which is exactly what is happening in the linear graph show in Figure 8.2 , can be simplified to 


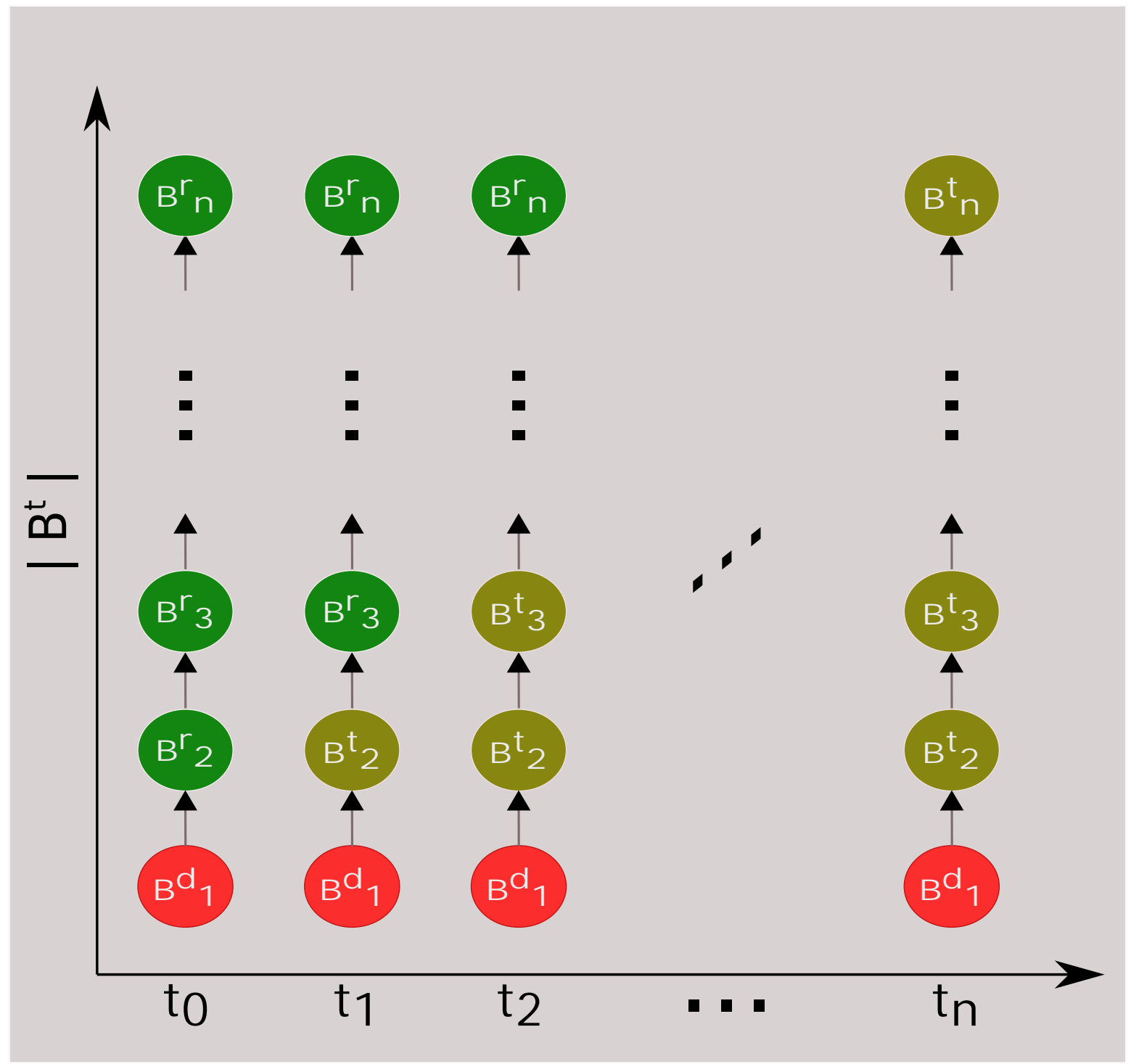

Figure 8.2: The simplified interaction graph. This figure shows from left to right the snapshots for the temporal evolution of the linear graph representing our bacterial population. The cell types are $B^{r}, B^{d}$ and $B^{t}$ standing respectively for recipients, donors and transconjugants.

$\beta=1 / \Delta_{t}$. Withal it is easy to realize that the slope have the following $\lim _{\Delta_{t} \rightarrow \infty} 1 / \Delta_{t}=0$ $\lim _{\Delta_{t} \rightarrow 0} 1 / \Delta_{t}=\infty$ lower and upper limits.

The Figure 8.2 may seems hard to interpret at a first sight but it is really simple and 


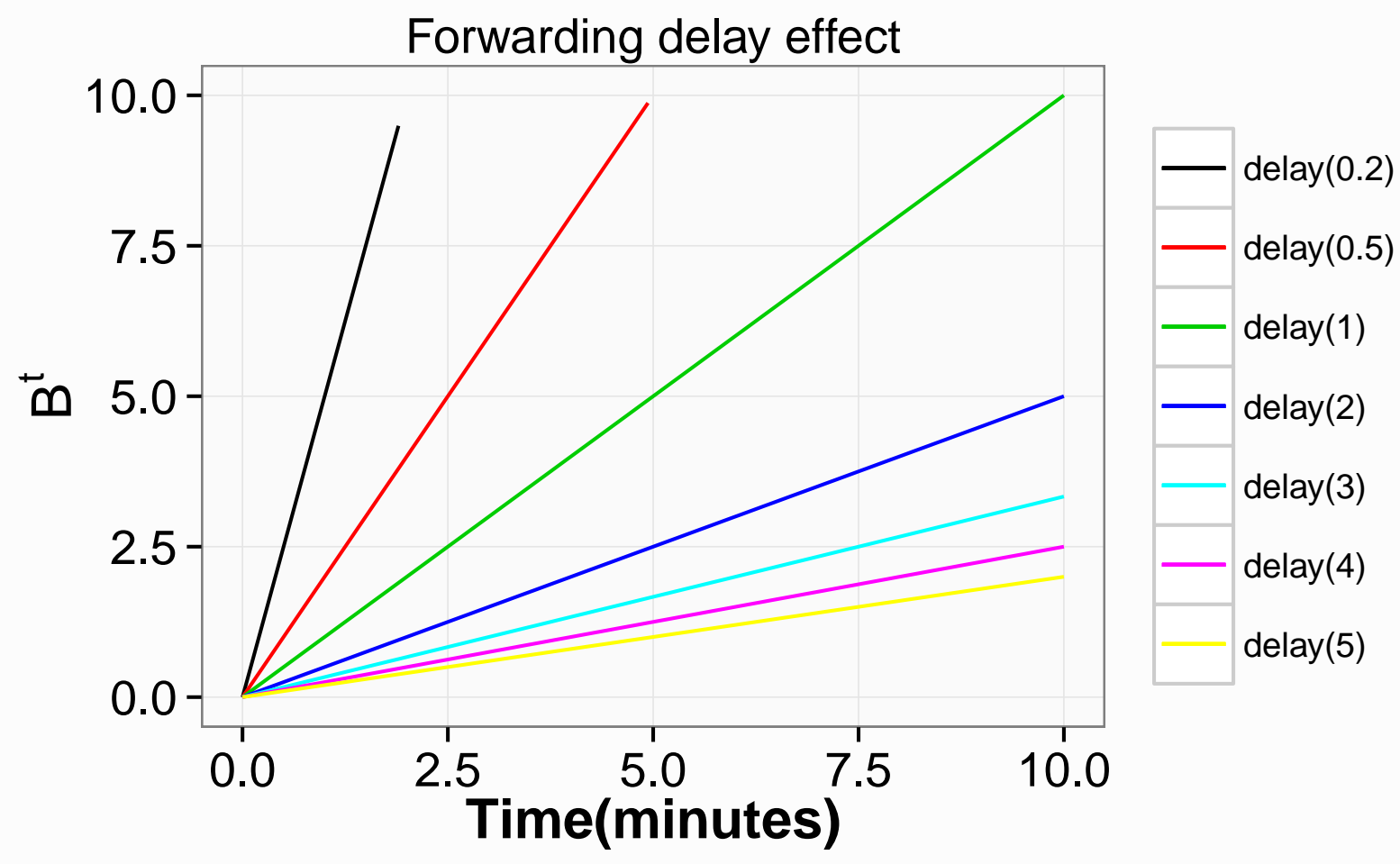

Figure 8.3: The effect of forwarding delay in infection speed. This graphic shows the effect of increasing delays in the plasmid forwarding network. As can be observed the slope is inversely proportional to the forwarding delay.

its objective is just to try to provide an explanation for why the time when conjugation happens plays a crucial role in the overall observed kinetics. That is the reason why we decided to isolate it from all other components which may difficult the understanding the effect on an exponentially growing colony. This figure simply shows a group of bacterial cells placed one after the other, being the first one, at $t_{0}$ a plasmid donor and the remaining just uninfected cells. As can be observed, at successive time points $\left(t_{1}, t_{2}, \ldots, t_{n}\right)$ one recipient bacterial cell $B^{r}{ }_{i}$ is infected by its predecessor becoming a transconjugant cell until the time point $t_{n}$ when all recipients are converted to transconjugants. The time points on the $x$ axis are arbitrary time intervals representing the total amount of time required by a bacterial cell $B^{t}{ }_{i}$ infect its successor neighbor $B^{t}{ }_{i+1}$ since the time the $B^{t}{ }_{i}$ have been infected by predecessor $B^{t}{ }_{i-1}$. 
The effects of reducing or incrementing the distance between these time points are shown on the Figure 8.3, where different intervals of $t_{i+1}-t_{i}$ are plotted together $\left(t_{i+1}-t_{i}=0.2,0.5,1.0,2.0,3.0,4.0\right.$ and 5.0). As can be seen, we show the straight lines representing how the distance between the time points of Figure 8.2 are affecting the slope of conjugation rate (in this case simply the number of transconjugant cells). This example may be a bit naïve but capture the essential aspect of the question, that is to say, increasing the time required for accomplish a conjugation round the slope of conjugation rate decreases. The same effect is observed in the Figures 7.4 and 7.5 shown in the previous chapter for an exponentially growing colony mixed with the effect of more factors.

\subsection{The effect of cell density on initial contact delay}

The initial inoculum concentration and the carrying capacity of culture medium available for bacterial cells, has vital implications for elucidating accurately the conjugation rates. These parameters together may be conditioning the in-silico experimental results. Both parameters are influential on the average distance between bacterial cells, which consequently affects the mating pair formation, the time required for the initial contact and how long takes for the first conjugative event be observed. In addition, the lag period also imposes a delay for the average time required for proficient donors have access to plasmid free cells.

A simple approximation to the problem of scaling how many bacterial cells disposed over a surface are equivalent to a volumetric measurement, is to consider the total carrying capacity of both and determine the scale factor which is given by the expression $N / L^{2}$, where $\bar{N}$ is the carrying capacity of liquids and $L^{2}$ the total amount of lattice sites [ZDF+12]. Thus, assuming the computational domain is a grid of $1000 \times 1000 \mu m$ with a carrying capacity of $10^{6}$ agents and that one milliliter of solution can hold approximately $10^{9}$ bacterial cells then, the scale factor would be $10^{9} / 10^{6}=10^{3}$. That approach has been used for scaling the results of end-point estimates [SGSL90] of 2D computational models to make them comparable with those values found on liquid cultures. The values reported for the average number of cells $/ \mathrm{ml}$ of solution present great variability ranging in orders of magnitude from $10^{7}$ to $10^{9}$ for studies using the E. coli $\mathrm{K}-12$ strain MG1655 [SJPD07]. Therefore, the actual values provided in the experimental 
setups must be taken with care as a reference for approximating the parameters of a computational model and not as absolute values.

Nonetheless, it is important to consider carefully the initial volume inoculated on the experimental plates alongside with the reference $O D_{600}$ values reported for the laboratory experiments being simulated. Both values are required for generating a realistic initial distribution of individuals over the computation domain. With these parameters plus the surface area of the devices where the inoculum will be plated, it is possible to generate some basic estimates for the initial average distance between individuals which are responsible for the time required for the occurrence of first conjugative event. One of the most straightforward ways of generating estimates for the average distance between individuals is using the relation between nearest-neighbor and population density [Bla58] which is given by the Equation (8.3)

$$
\bar{r}=\sqrt{\frac{1}{4 m}}
$$

where $\bar{r}$ is the average distance between individuals and $m$ the population density expressed as the number of individuals per surface area. The average distance will be used in the computation model to distribute the initial population aiming to mimic the existing real separation between bacterial cells.

The value of density $m$ can be calculated by the expression shown in Equation (8.4)

$$
m=O D 600 * I / S * C * 1 / S
$$

where the term OD600 depicts the density readings in cells $/ \mathrm{ml}$, the next term $I$ is the volume of solution in $\mu L$ inoculated on the experimental plates, the variable $S$ represents the surface of experimental plates in squared centimeters and finally $C$ is the conversion factor to express the value of density with the appropriated surface unit, thus for expressing $m$ in cells $/ \mathrm{mm}^{2} \mathrm{C}$ must be equal to $10^{-} 5$.

Using the data of a conjugation assay for determining the conjugation rates in solid surfaces[ $\left[\mathrm{dCRC}^{+}{ }^{12}\right]$ we can illustrate the idea presented above. This experimental setup uses 24-well microtiter plates which have a surface of $1.9 \mathrm{~cm}^{2}$ which have been inoculated with $15 \mu \mathrm{L}$ of a cell solution of approximately $6 \times 10^{8} \mathrm{cells} / \mathrm{ml}$ given by an OD600 of 0.6 units. Therefore, the value of $m$ is calculated as shown in Equation 


$$
m=6 \times 10^{8} \text { cells } / m l * 15 / 1,9 * 10^{-} 5 * 1 / 1.9
$$

which give that $m$ is approximately 25000 cells $/ \mathrm{mm}^{2}$. Introducing that value in Equation (8.3) we have

$$
\bar{r}=\sqrt{\frac{1}{4 \times 25000}}=0.003 \mathrm{~mm}
$$

converting to micrometers $\left(0.003 * 10^{3}\right)$ we have an average initial distance between individuals equal to $3 \mu \mathrm{m}$. If we increase one order of magnitude the value of initial concentration of cells from $6 \times 10^{8} \mathrm{cells} / \mathrm{ml}$ to $6 \times 10^{9} \mathrm{cells} / \mathrm{ml}$ we would obtain an average distance between individuals equal to $r=0.001$ or $1 \mu \mathrm{m}$ of separation between bacterial cells.

The overall effect of using different initial densities in the separation of bacterial cells can be seen in the virtual culture plates presented in Figure 8.4. As can be observed, the subfigures $(a)$ to $(c)$ shows visually the effect of decreasing the initial concentration of bacterial cells in the increment in the average distance between individuals distributed randomly over a culture plate. The Subfigures 8.4a, 8.4b, 8.4c and $8.4 \mathrm{~d}$ are respectively the virtual plates for initial densities of $10^{9}, 10^{8}, 10^{7}$ and $10^{6}$.

The quantitative effects of varying the initial density of bacterial cells in the required time for engaging the first conjugative contact, is shown in Figure 8.5 which contains the simulation results of an experimental setup consisting in $30^{1}$ replications keeping fixed all parameters but the initial cell density (the model parameter $N_{0}$ ). The curves are the average of this 30 replications for each initial density ${ }^{2}$. Additionally, the Figure also shows the curve for the reference experimental data. As can be easily observed, the effect of initial density on the time required for making the cell-to-cell contact and consequentially the conjugative transfer activity is quite evident. The initial densities of $10^{9}$ and $10^{8}$ implies a minimal initial distance between cells in such extent the conjugative activity takes places almost in one generation time as can be seen in the first two curves from left to right. The reference data [ $\left.\mathrm{dCRC}^{+12}\right]$ shows conjugative activity

\footnotetext{
${ }^{1}$ The number of 30 replication has been chosen taking into account the model output stability which has been previously discussed in this work.

${ }^{2}$ The complete $\mathrm{R}$ code for this experiment is available on the Appendix $\mathrm{A}$
} 


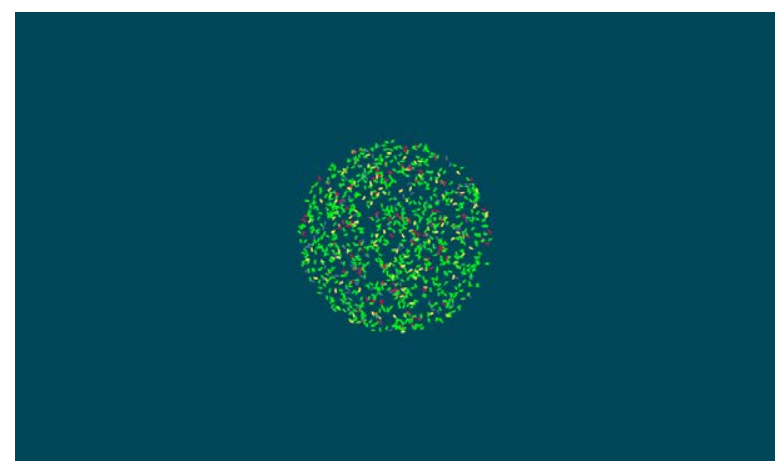

(a) Initial density $5 \times 10^{9}$

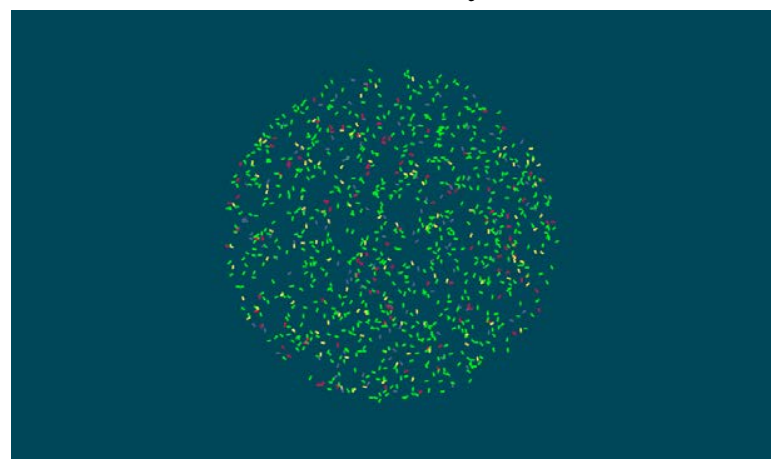

(c) Initial density $5 \times 10^{7}$

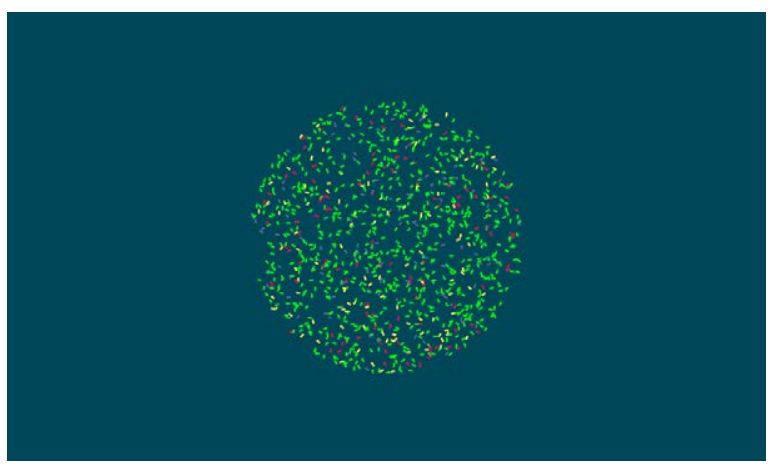

(b) Initial density $5 \times 10^{8}$

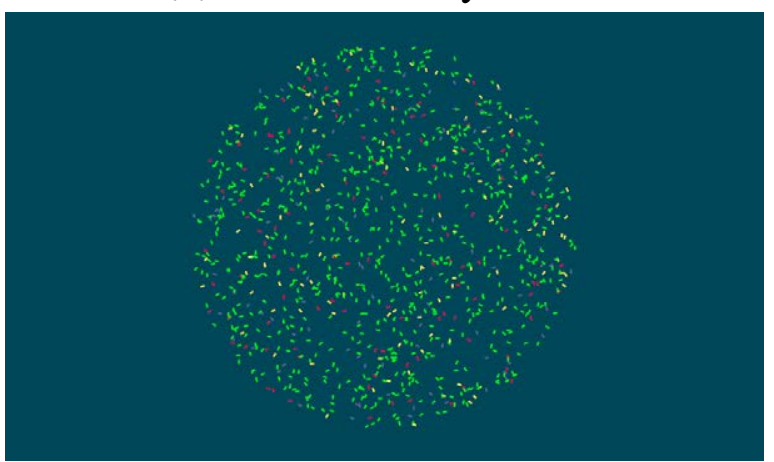

(d) Initial density $5 \times 10^{6}$

Figure 8.4: Comparing the effect of initial density on the average distance between individuals in a bacterial culture.

(a conjugation rate $\mathrm{T} /(\mathrm{T}+\mathrm{R})$ higher than 0.75$)$ approximately from 120 minutes. The reference data is flanked by the curves simulating the initial densities of $10^{7}$ and $10^{6}$ at left and right respectively. In the last experiment, the conjugative activity is observed very late, approximately on the 190 minutes of simulated time which is roughly more than four generations.

\subsection{Description of model analysis}

In order to fully validate the structural aspects of how we have modeled the conjugation in our individual-based model BactoSIM, a throughout analysis has been carried out using the methodological approaches previously described in this work, namely the sensitivity analysis and the parameter estimation. Therefore, three sensitivity analysis experiments have been performed, for exploring the following base parametrization 


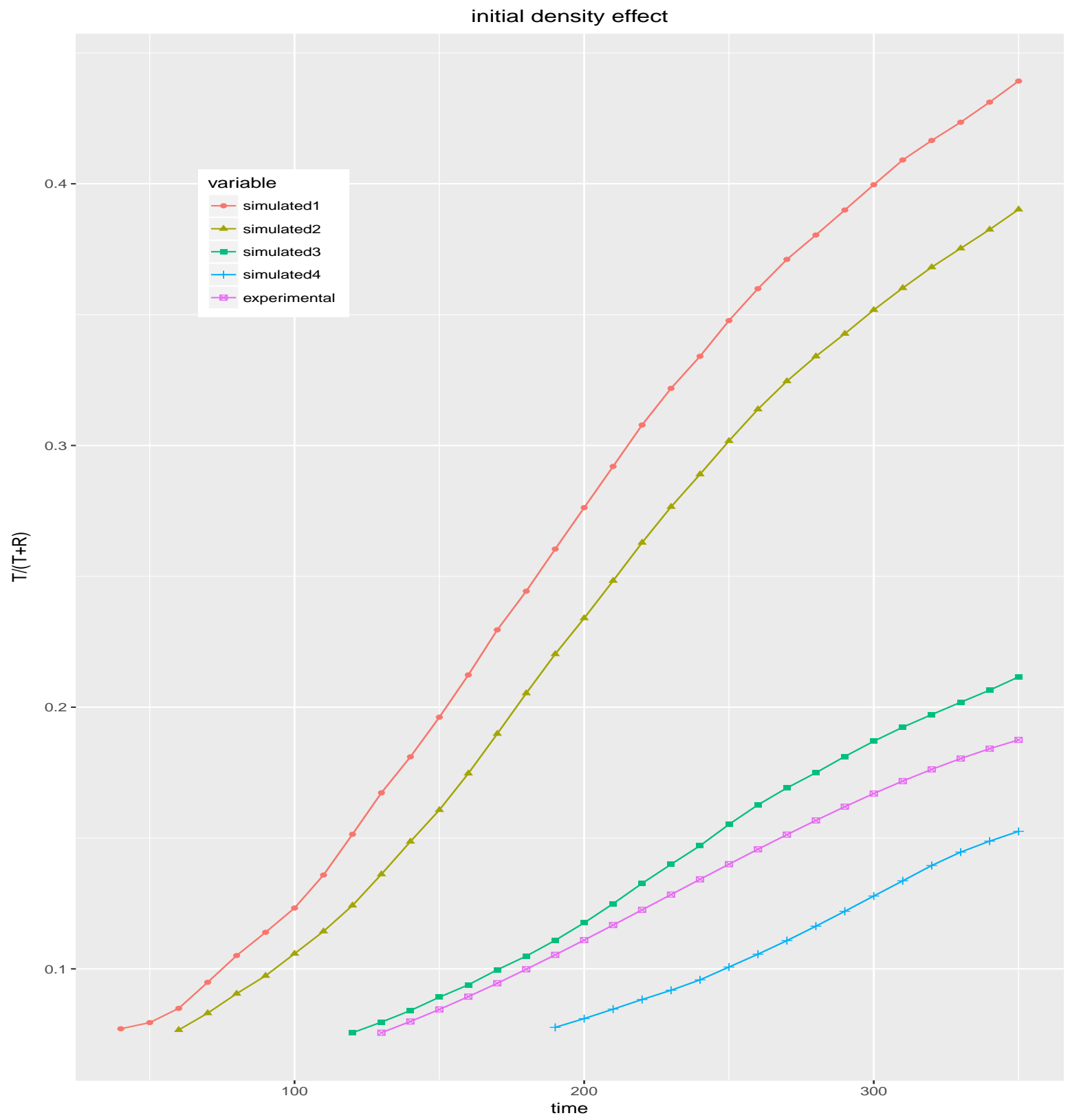

Figure 8.5: The effect of varying the initial densities $\left(N_{0}\right)$ on the conjugation rates, showing how it affects the time required for the initial contact. These curves represents, from left to right respectively the simulation output of using $N_{0}=10^{9}$ (red), $N_{0}=10^{8}$ (olive green), $N_{0}=10^{7}$ (green), the curve fitted to the experimental data (magenta) and finally, the simulation output of using $N_{0}=10^{6}$ (blue). 
options:

- Case 1 - Study of repressed plasmids.

- Case 2 - Study of non-repressed plasmid.

- Case 3 - Study of non-conjugative mobilizable plasmids.

The objective of differentiate these three approaches is owing to the fact that the model follow distinct trajectories for each of these initial cases and the impact of some model parameters on the model output variability are not the same depending on the type of plasmid being simulated. For instance, in the Case 1 we have an additional parameter which allow to specify the initial ratio of donors which are de-repressed and conjugative proficient and obviously it has no meaning for initially non-repressed plasmids. These updates have not been made on the model structure itself, but instead they are just slight superficial modifications on the required input parameters for exposing some previously fixed values or separating the conjugation model from the cell cycle allowing them to be evaluated using the sensitivity analysis techniques depicted on the chapter 6. Consequently, the input parameters which are assessed here are the same already presented previously on the chapter 7 but including the new initialization options included in the Table 8.1.

Table 8.1: The list of BactoSIM additional model initialization parameters.

\begin{tabular}{lll}
\hline Parameter & Unit & Description \\
\hline model & N/A & $\begin{array}{l}\text { The model type. Accepted values are 1,2 or } 3 \text { which } \\
\text { are respectively the previously discussed models for } \\
\text { conjugation. }\end{array}$ \\
$\begin{array}{lll}\text { nutrients } \\
\text { initialDRDratio }\end{array}$ & N/A & $\begin{array}{l}\text { The amount of nutrient particles. } \\
\text { The ratio of initial donors which are derepressed. }\end{array}$ \\
\hline
\end{tabular}

\subsection{Preliminary screening of model output}

In this section we will show the preliminary results obtained for the sensitivity analysis process which have been used for evaluate and understand the outputs generated by 
the BactoSIM model in order to substantiate some conclusions of this work. Thus, the first aspect we want to verify, is whether the modeling approach for representing the conjugation has some significant impact on the model outcome as has been visually evaluated in the previous chapter. Bearing this in mind, we have applied the screening method of Morris for an initial approximation to the problem, evaluating the effect of model choice in for the two main cases mentioned in the previous section, namely the case 1 which represents the plasmids naturally repressed and the case 2 which encloses those plasmids which are constitutively expressing the conjugative genetic machinery.

The main objective of using an screening method, as have been mentioned previously in the chapter 6 , is to undertake an initial delimitation of the factors which are responsible for the most of the output variance. In this specific case we want to verify whether or not the model is an important factor. For ensuring the reproducibility of the results shown here, the scripts used for each of the experiments discussed in this chapter will be listed in the Appendix A.

The results shown in Table 8.2, in Table 8.3 and in Table 8.3 are the metrics $\mu$, $\mu^{*}$ and $\sigma$ calculated for the elementary effects which have been estimated using the method of Morris. These values allows to rank which factors are causing the most of variance for the rate $(T /(T+R)$ output variable. The results are sorted by the $\mu *$ metric from the most to the less significant factors. As can be observed the model input parameter has a very significant effect and is above all others remaining parameters for the three cases of study, namely the case 1 , case 2 and case 3 , respectively the model for repressed, de-repressed and mobilizable plasmids. 
Table 8.2: The Morris metrics for the elementary effects of BactoSIM model. This table contains the results for the case 1 .

\begin{tabular}{lrrr}
\hline Input parameter & $\mu$ & $\mu^{*}$ & $\sigma$ \\
\hline model & -0.151139889 & 0.16720867 & 0.44574069 \\
conjugationCost & 0.030000238 & 0.03398144 & 0.07126060 \\
$\gamma_{0}$ & 0.020518046 & 0.02849222 & 0.06534011 \\
nutrients & 0.020736414 & 0.02277778 & 0.04429573 \\
cyclePoint & -0.001234407 & 0.01706222 & 0.03081159 \\
initialDRDratio & 0.007167759 & 0.01545636 & 0.03506770 \\
pilusExpressionCost & 0.014500096 & 0.01453345 & 0.02439932 \\
\hline
\end{tabular}

It is interesting to recall that the model types differs fundamentally by the timing when conjugation is triggered and that the values for Morris metrics are very similar which seems to confirm, in some extent, the previous results, obtained from a simple visual assessment, which were pointing in the same direction.

Table 8.3: The Morris metrics for the elementary effects of BactoSIM model. This table contains the results for the case 2 .

\begin{tabular}{lrrr}
\hline Input parameter & $\mu$ & $\mu^{*}$ & $\sigma$ \\
\hline model & 0.032421289 & 0.07310568 & 0.11969663 \\
cyclePoint & 0.017944503 & 0.04340028 & 0.06430294 \\
nutrients & 0.032973536 & 0.03821871 & 0.03778982 \\
initialDRDratio & 0.002396539 & 0.03696602 & 0.05696925 \\
pilusExpressionCost & -0.032051696 & 0.03267854 & 0.03101232 \\
conjugationCost & -0.025976738 & 0.03093565 & 0.04885718 \\
$\gamma_{0}$ & 0.018859565 & 0.02691402 & 0.02644715 \\
\hline
\end{tabular}


Table 8.4: The Morris metrics for the elementary effects of BactoSIM model. This table contains the results for the case 3 .

\begin{tabular}{lrrr}
\hline Input parameter & $\mu$ & $\mu^{*}$ & $\sigma$ \\
\hline model & 0.037685503 & 0.05526285 & 0.09896928 \\
cyclePoint & 0.019857680 & 0.04926191 & 0.07241082 \\
initialDRDratio & 0.003399129 & 0.03650807 & 0.04455440 \\
$\gamma_{0}$ & 0.016580862 & 0.02704785 & 0.03759677 \\
nutrients & 0.025812398 & 0.02581240 & 0.03260287 \\
conjugationCost & -0.013923998 & 0.01916376 & 0.02748788 \\
pilusExpressionCost & -0.018641321 & 0.01921991 & 0.02054882 \\
\hline
\end{tabular}

Figures 8.6, 8.8 and 8.10 shows graphically the numeric results for the metric $\mu$ of the screening exercise which has been previously shown in the Tables 8.2 and 8.3 . Additionally, the metric $\mu^{*}$ is shown in Figures 8.7, 8.9 and 8.11. Both metrics $\mu$ and $\mu^{*}$ are taken from the elementary effects for model output representing the simulated conjugation rate $T /(T+R)$ for a repressed, de-repressed and mobilizable plasmids, the case 1, case 2 and case 3 respectively. These plots allows the easy visualization the effects of model choice in the variability of conjugation rate output.

The results seem to be consistent with the assumption that the approach used for representing the conjugation process, is clearly responsible for the most of the model output variation. In other words, the input parameter model type, which allows the selection of the conjugation strategy, is clearly and consistently ranked as the most important according to the results of Morris method. It means fundamentally that temporal structure of the process (the time when the conjugative transfer is most likely to happen) is responsible for the most part of the global dynamics. It is necessary to mention that the Morris method is not estimating the model parameters but just ranking the effects of varying these parameters.

In the case of the derepressed plasmid, the other parameters in importance are the nutrient availability, the $\gamma_{1}$ (cyclePoint) and the $\gamma_{0}$ falling all very close in the rank but $\gamma_{1}$ is the input parameter of this group with the greater $\sigma$ which means a high effect of 
variating the parameter input values. The mobilizable plasmid shows a similar results with $\gamma_{1}$ and nutrient availability ranking high but having the first a higher value for $\sigma$ which, as we have mentioned previously indicates that the variation on the values of this input parameter causes a large variability in the estimated elementary effects and consequently on the model output.

On the other hand, for the repressed plasmid, we may observe a group of three parameters with high importance which are the frequency of initial derepressed donor ratio in the population (initialDRDratio), the model type and the metabolic cost of expression the conjugative machinery (pilusExpressionCost). The parameter model type has the higher value of $\sigma$ which means that the model output will be very sensible to variations on its values. This group is followed by the conjugatioCost which also shows a high value of $\sigma$. It is important to mention that the differences observed for the (pilusExpressionCost) in the metrics $\mu$ and $\mu *$ (see Section 6.1.2) are a possible indication of effects of different signs which could indicate a non-monotonic ${ }^{3}$ effect on the output. The effect of (pilusExpressionCost) possibly depends non-linearly on other model components, mainly those affecting the initial number of transconjugant cells, such as the initial derepressed ratio.

Finally, despite of not being a formal statistics hypothesis test, the results of Morris screening method can serve as reference for discarding, with relative safety that the way the conjugation rule is implemented with respect to the timing aspects, are not equal. The Morris method allows the input parameters of a model to be ranked in order of its importance using the metric known as elementary effects. Therefore, we are not directly testing whether $\mathrm{M} 1, \mathrm{M} 2$ and $\mathrm{M} 3$ are equal or not but ranking the effects of setting this input parameter. Indirectly, if the model type has no effect, it should have been ranked low, but it is not the case. Hence, we may affirm that model type (how conjugation rule is implemented) has an effect on the model output. We will extend this observation applying a formal hypothesis test approach in the next section.

\footnotetext{
${ }^{3}$ We mean by non-monotonic effect, that the model output does not follows a linear relation with the input parameter domain. In other words, increasing some input parameter value may cause the model output to increase for a specific set of values and decrease for others. This normally indicates that model contains feedback loops and non-linear components.
} 
The hypothesis we are analyzing in this work is presented on the box below. The null hypothesis basically says that the model formulation (the model type is a parameter for choosing one of the strategies presented in the previous chapter) has no effect being all approaches equivalent. According to the model description presented previously, in the M1 (strategy 1) the conjugative transfer may occur as soon a donor cell finds a suitable plasmid free recipient. In the M2 (strategy 2) the conjugation is uniformly distributed along the cell cycle and finally in the M3 (strategy 3 ) the conjugation is assumed to occur in a precise point of time, specified by the parameter $\gamma_{1}$ (cyclePoint).

\section{Hypotheses}

$h_{0}, \mathcal{M}_{1}=\mathcal{M}_{2}=\mathcal{M}_{3}$ $h_{1}, \mathcal{M}_{1} \neq \mathcal{M}_{2} \neq \mathcal{M}_{3}$ 


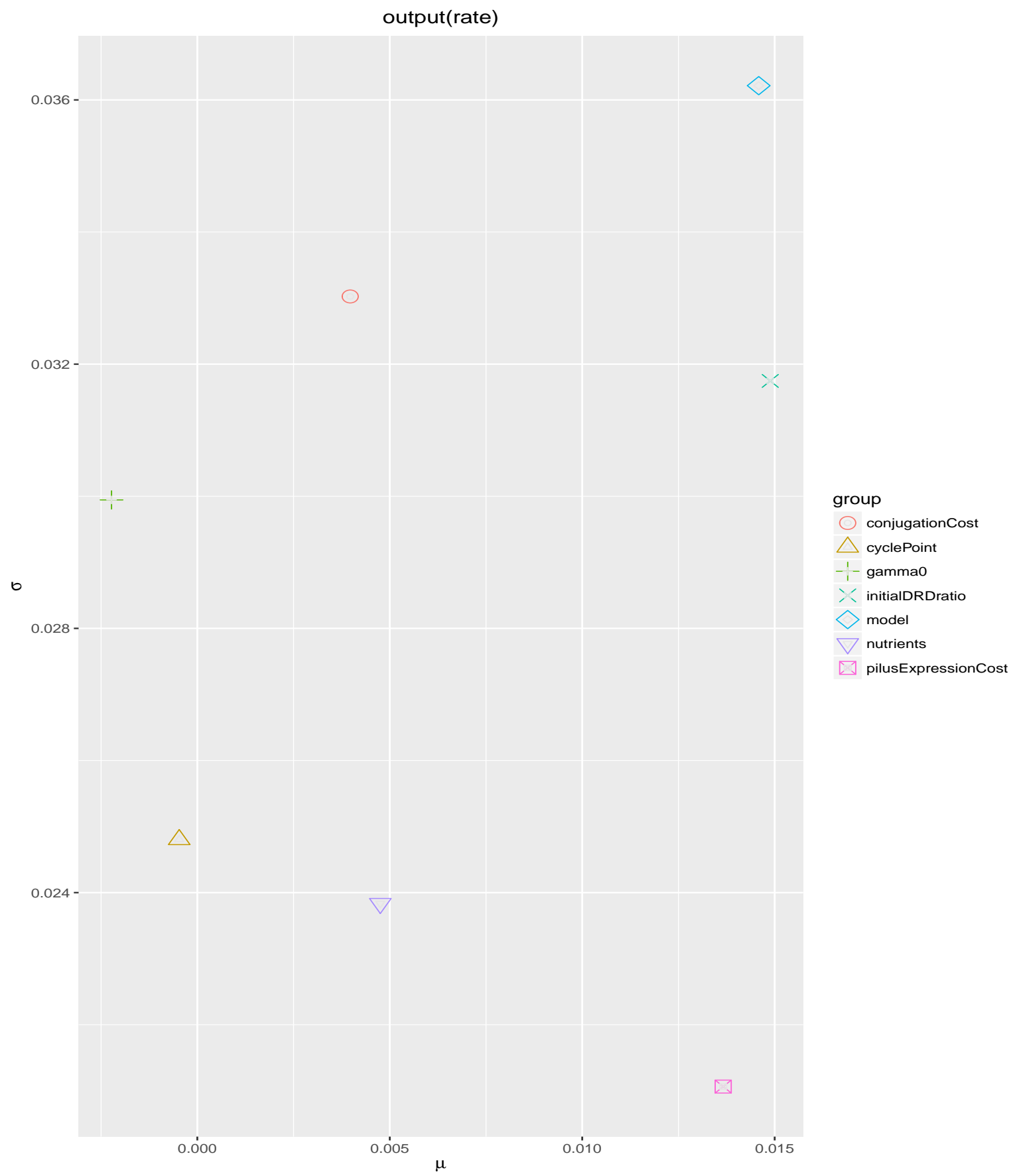

Figure 8.6: Results of Morris screening method for the BactoSIM output simulating a repressed plasmid (case 1 ). This figure shows the $\mu$ sensitivity metric for BactoSIM $T /(T+R)$ output. 


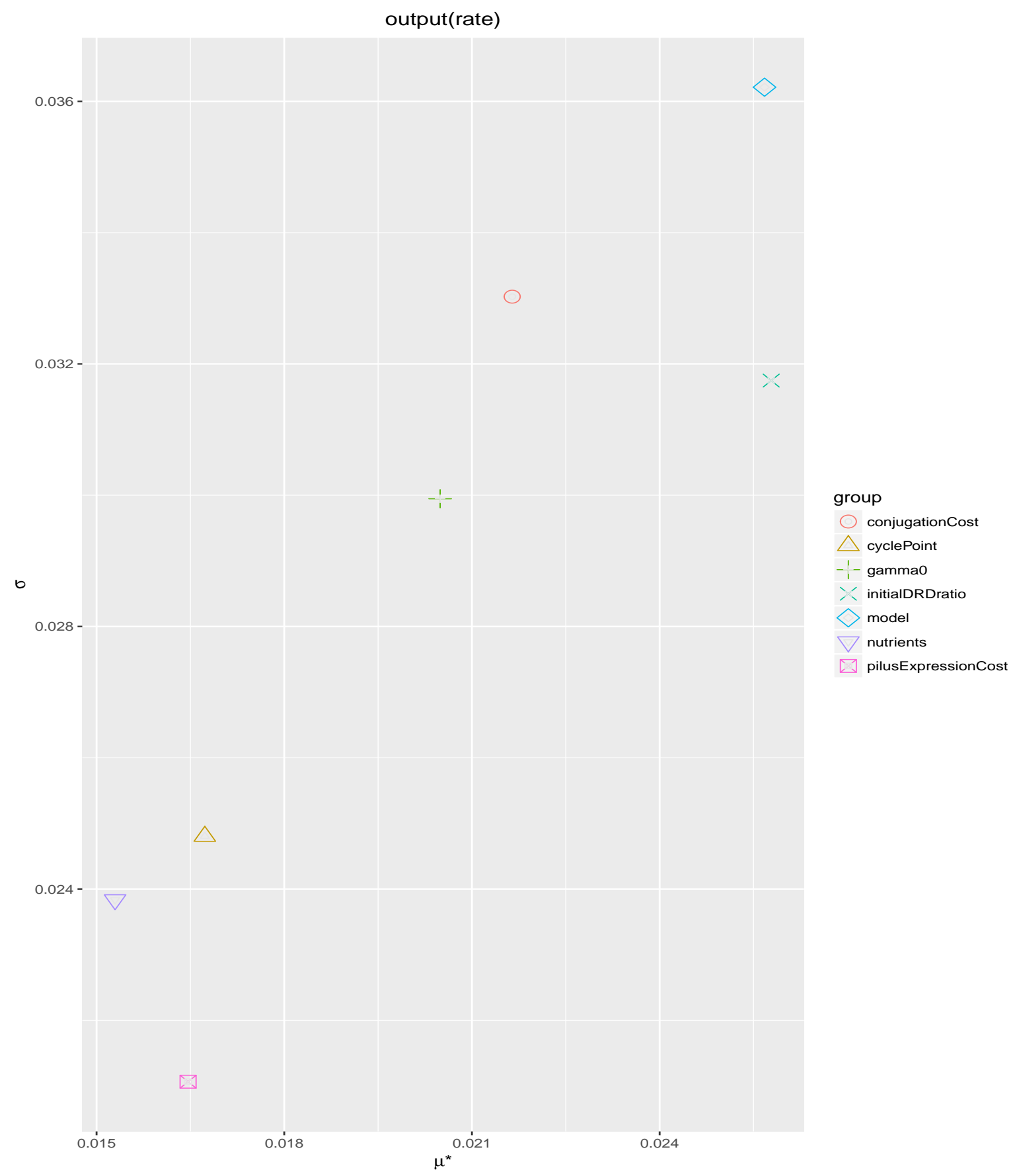

Figure 8.7: Results of Morris screening method for the BactoSIM output simulating a repressed plasmid (case 1 ). This figure shows the $\mu^{*}$ sensitivity metric for BactoSIM $T /(T+R)$ output. 


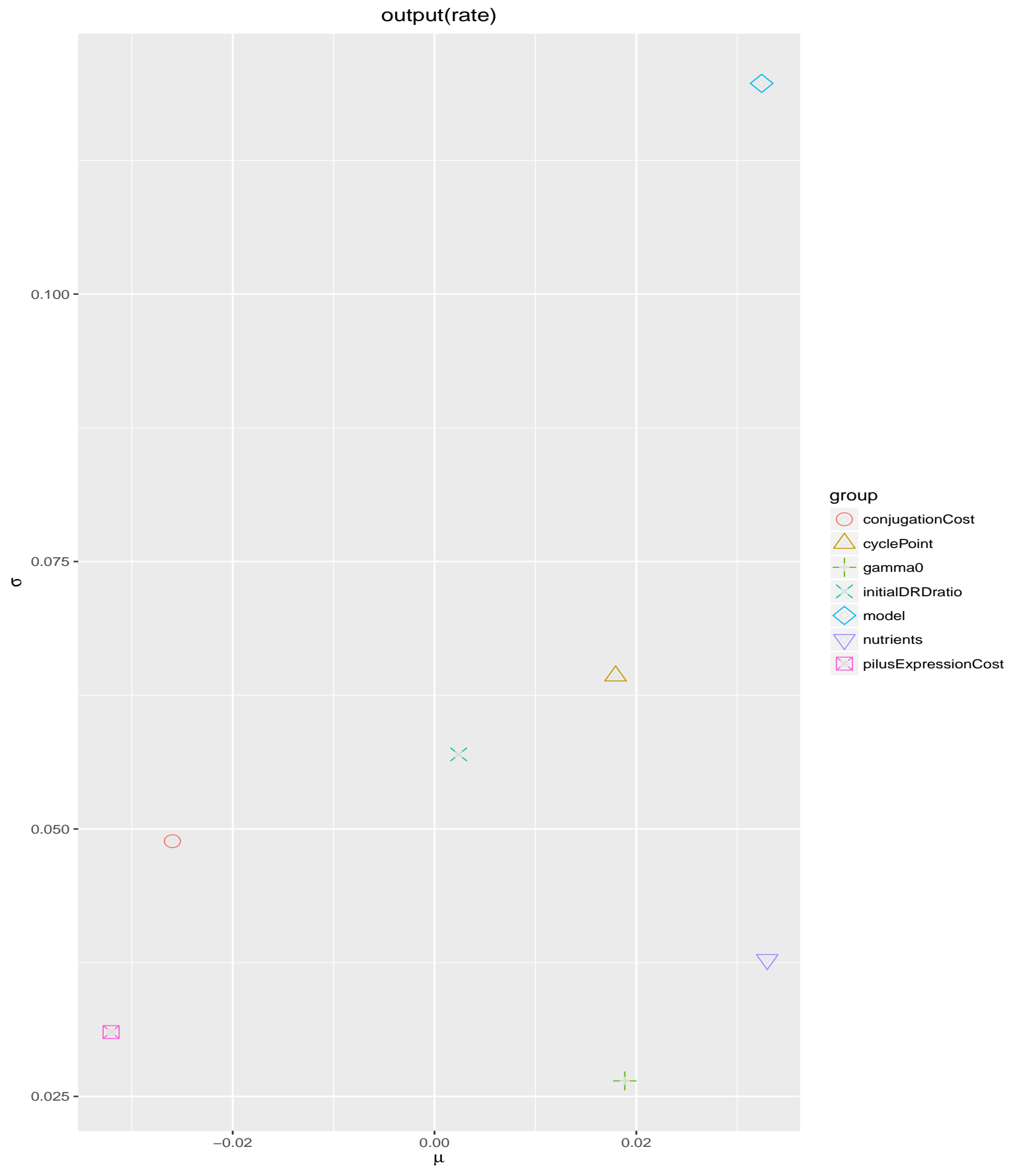

Figure 8.8: Results of Morris screening method for the BactoSIM output simulating a derepressed plasmid (case 2). This figure shows the $\mu$ sensitivity metric for BactoSIM $T /(T+R)$ output. 


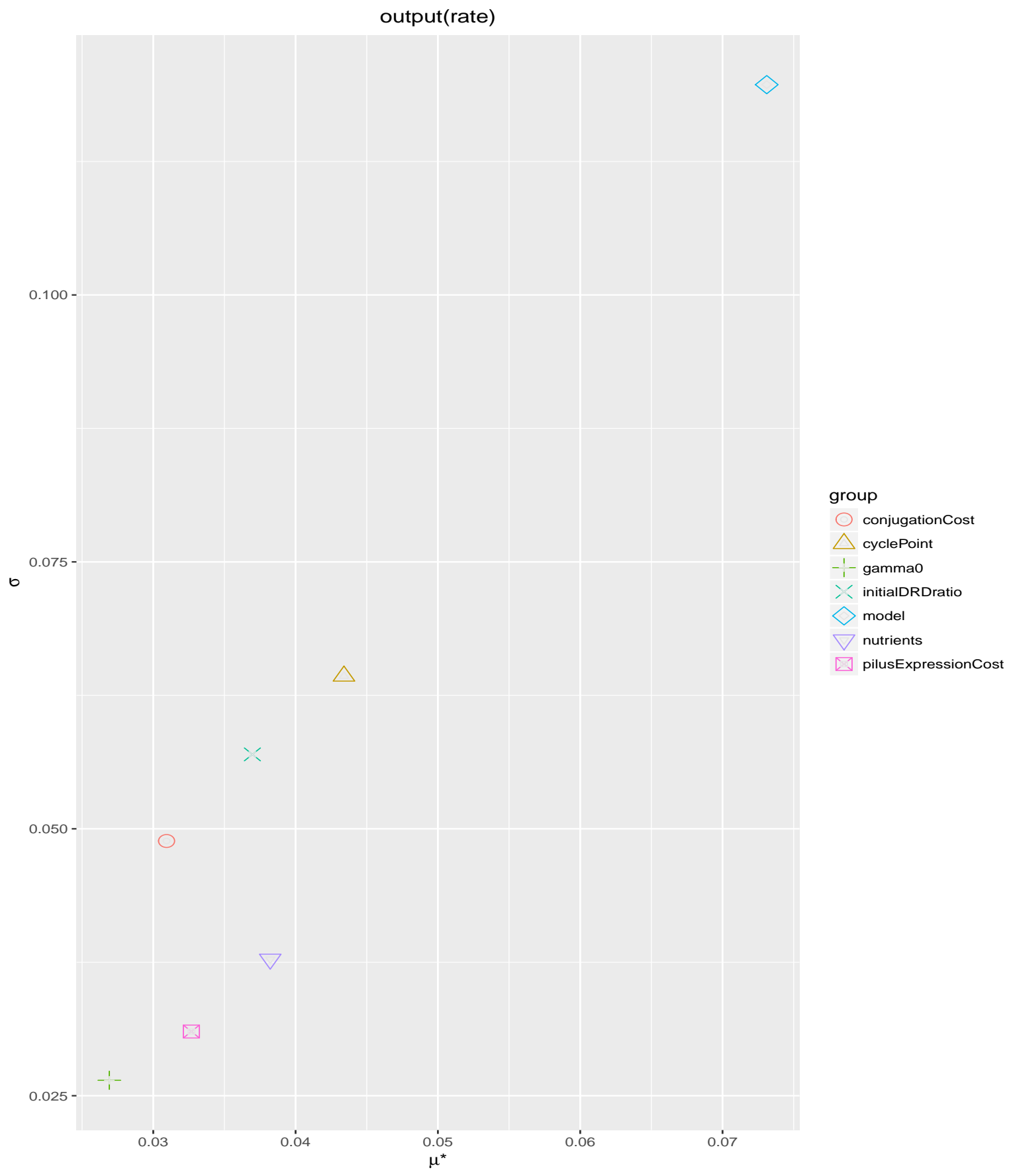

Figure 8.9: Results of Morris screening method for the BactoSIM output simulating a derepressed plasmid (case 2). This figure shows the $\mu^{*}$ sensitivity metric for BactoSIM $T /(T+R)$ output. 


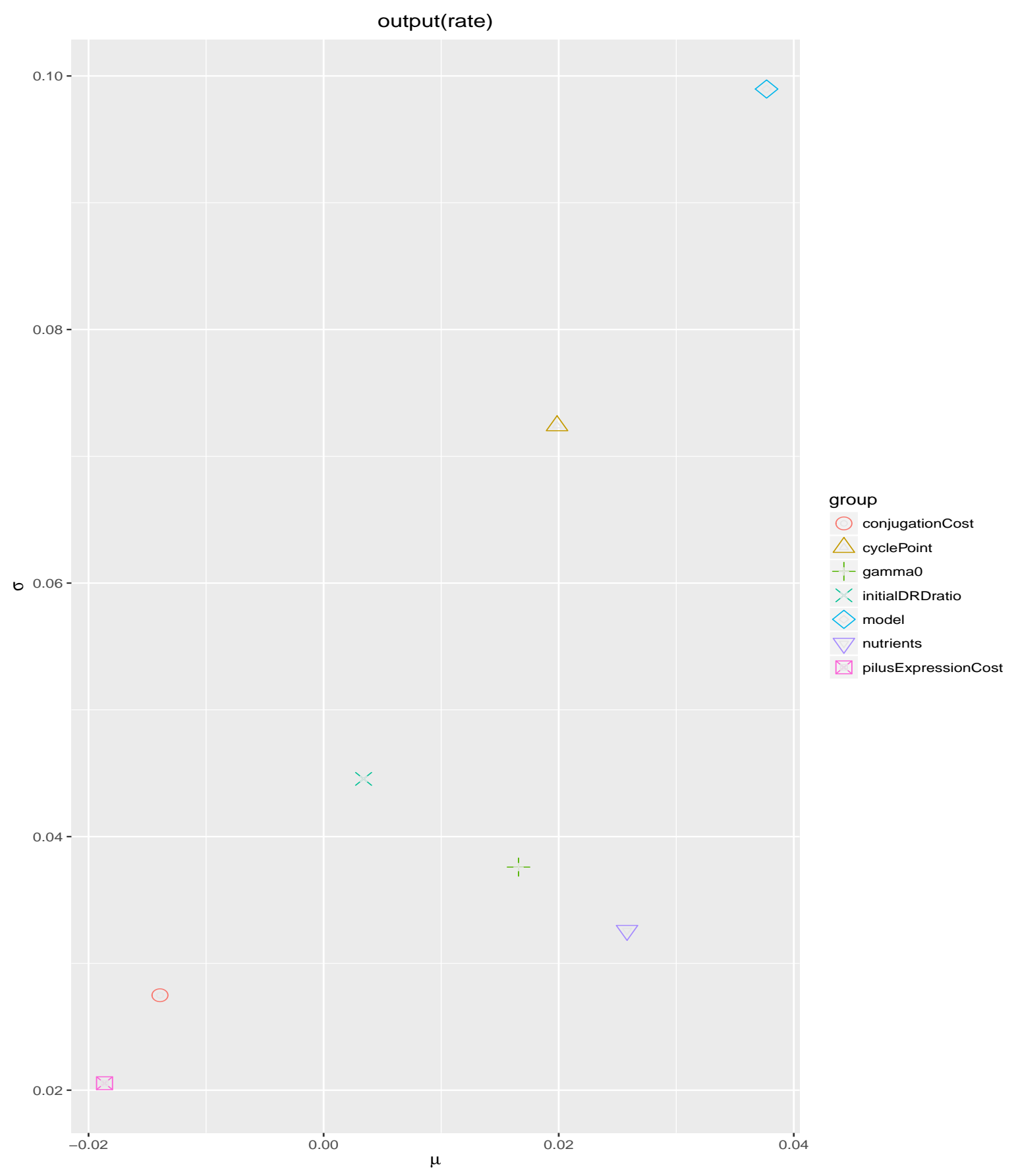

Figure 8.10: Results of Morris screening method for the BactoSIM output simulating a mobilizable (OriT) plasmid (case 3). This figure shows the $\mu$ sensitivity metric for BactoSIM $T /(T+R)$ output. 


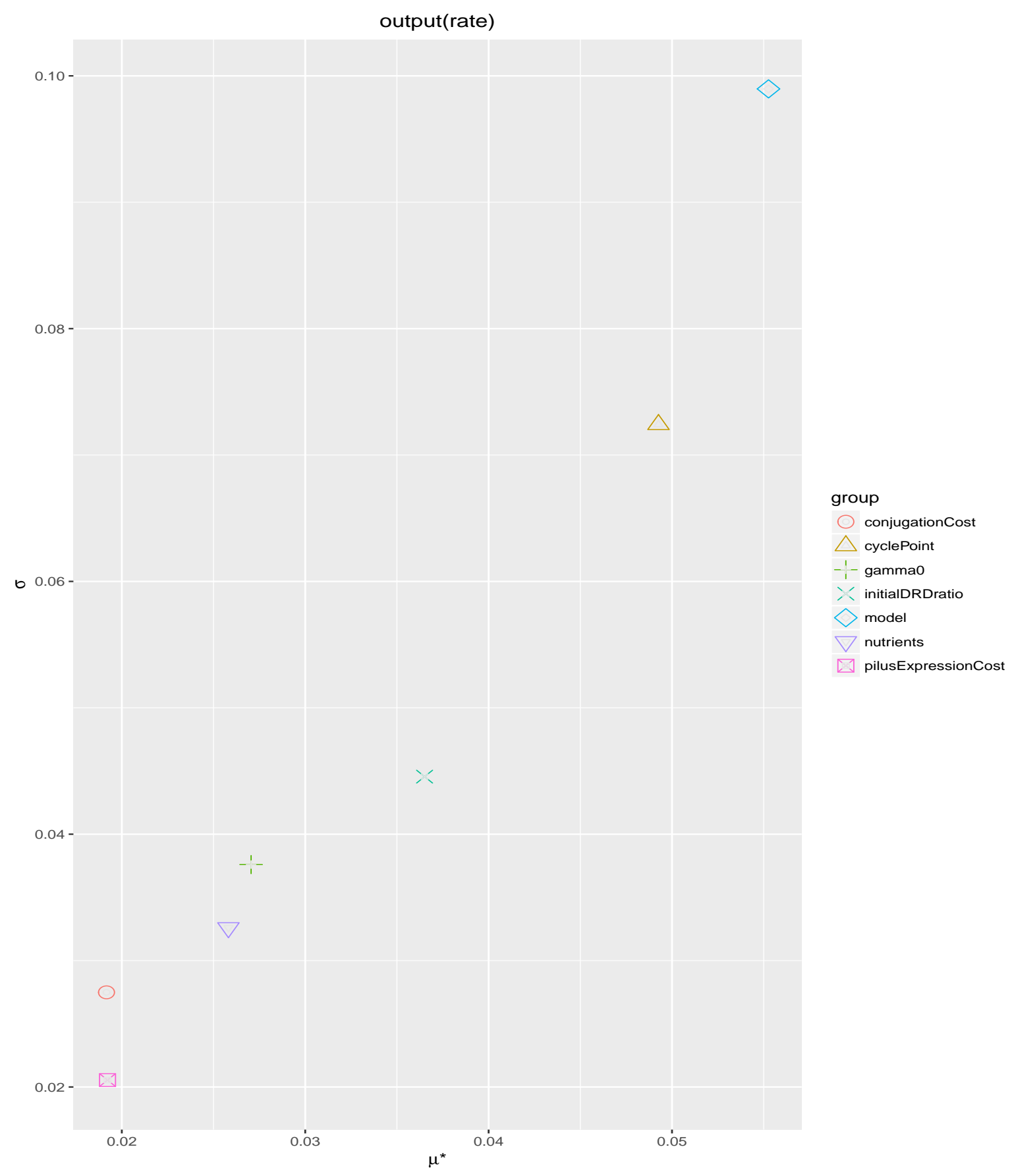

Figure 8.11: Results of Morris screening method for the BactoSIM output simulating a mobilizable (OriT) plasmid (case 3). This figure shows the $\mu^{*}$ sensitivity metric for BactoSIM $T /(T+R)$ output. 


\subsection{Assessing the fitness of different models}

In the previous section we have found that the parameter model type (M1, M2 and M3) significantly affects the behavior of conjugation rate estimated by the BactoSIM model. The model type is the input parameter which allows the selection of modeling strategy defined in Sections 7.3 and 8.3 the possible values are 1, 2 and 3 respectively for $M 1$, M2 and M3. In this section we will undertake a parameter estimation study, separating the analysis in three groups keeping fixed the model type for each of them in order to provide a comprehensive view about which could be the possible best approach for representing the bacterial conjugation. For undertake the parameter estimation process, we will use the EvoPER package described previously in the chapter 5 . We have used initially the simulated annealing algorithm for the parameter estimation owing to the fact that it requires fewer model evaluations than other choices and provide good approximations to the model optima. The scripts for running the parameter estimation and the data analysis of this section are provided in Appendix A.

The Table 8.5 shows the fitness cost of each model type as well as the averaged values of the solutions found for each of the three approaches for modeling the conjugative transfer. The best values for the optimization criteria was achieved by the model 3 followed by the model 2 and model 1 . On the other hand, when considering the average values of cost functions the models 2 and 1 change its order but the model 3 still is the best positioned. In other words, we have applied an optimization algorithm to find the best combination of parameters for the three strategies/model types which have been fully described in the ODD protocol of BactoSIM model presented in the Section 7.3. Roughly speaking, the process consists in keeping fixed the parameter which allows the selection of the model/strategy (this input parameter is called model and may have three values 1,2 and 3) and allowing all other parameters to vary over their domains, repeating the operation for the model $=1,2,3$. With this approach we have found the possible best combination for each of them which shows that the strategy/model 3 provided the minimal distance between experimental and simulated data.

The word fitness or the expression fitness cost used in this section is unrelated to 
the evolutive meaning of the term. Here, the term fitness basically means a function to be optimized, which in most cases means to find the function minima. Hence, our fitness cost function is distance measurement between the experimental data for the conjugation essays and the simulated results generated with our BactoSIM model. Therefore, in order to find the best parametrization for our model we want to minimize the fitness function.

Table 8.5: The results for parameter estimation of BactoSIM using the simulated annealing metaheuristic. The table shows estimated best values satisfying the optimization criteria. The estimated model parameters shown in this table are $\gamma_{1}, C_{1}, C_{2} \gamma_{0}$ and ratio $(d r d)$, respectively the cell cycle point, the conjugation cost, the t4ss pilis expression cost, the intrinsic conjugation rate and the ratio of donors which are initially de-repressed.

\begin{tabular}{lrrrrrrr}
\hline Model & fitness & average & $\gamma_{1}$ & $C_{1}$ & $C_{2}$ & $\gamma_{0}$ & ratio $(d r d)$ \\
\hline model 1 & 4.551496 & 9.55 & 0.0 & 43.38 & 0.84 & 0.45 & 0.05 \\
model 2 & 4.489114 & 12.94 & 50.0 & 0.17 & 6.99 & 1.02 & 0.01 \\
model 3 & 2.618539 & 6.88 & 81.14 & 7.56 & 62.04 & 1.94 & 0.08 \\
\hline
\end{tabular}

It is also worth to note in Table 8.5 that the parameters estimated for the models 1 and 2 shown values for the metabolic cost which could be inconsistent with the experimental reality. For instance, it was estimated that the conjugative cost should be approximately $43 \%$ and $0.17 \%$ respectively for model 1 and 2 . The model 1 shows a value for the pilus expression cost inferior of the conjugation cost which does not match with the expected behavior. On the other hand the model 3 shows very consistent estimations for cell cycle and for both cost which possibly are very close to the real values. 
Table 8.6: The Anova results for verifying if the values estimated by the different models are statistically significant.

\begin{tabular}{lrrrrr}
\hline & Df & Sum Sq & Mean Sq & $F$ value & $\operatorname{Pr}(>F)$ \\
\hline model & 2 & 3584.41 & 1792.20 & 34.40 & $3.65 \mathrm{e}-14$ \\
Residuals & 297 & 15472.31 & 52.10 & & \\
\hline
\end{tabular}

In order to verify that the null hypothesis can be reject we have applied the ANOVA test to the estimated fitness values. The $h_{0}$, as have been stated previously, is that all alternative model formulations (model/strategy 1,2,3) are fundamentally equal and therefore the $\gamma_{1}$ plays no role in such extent that conjugation may take place in any point of bacterial cell cycle.

The results of this test is shown in table 8.6 and in addition we have also performed a non-parametric of Kruskal-Wallis for avoiding to rely on any restrictive assumption about the distribution of the fitness values. This test have given a $p$ value of $2.448 e-11$, therefore in both case we can reject safely the null hypothesis.

It is worth noting that normally we should not rely exclusively on statistical tests for assessing the outputs of computational models. It is considered that ANOVA significance tests are not well suited for analyzing the effects alternative model formulations or for quantifying how significant are the contribution of the input factors on the model output [WRS ${ }^{+14}$ ] for two important reasons. First, they claim that the null hypothesis is known a priori to be false $4^{4}$ which violates the premise underlying the statistical tests which dictates that the outcome for the hypothesis under study is unknown. The second point is that the $p$-values are dependent on the number of model replication and consequently, with a high number of simulation replicates, low $p$-values are achieved leading (incorrectly) to take some effect as highly significant. In any case the significant test present here are simply a comparison for the average values for the model

\footnotetext{
${ }^{4}$ We cannot completely agree with this assumptions in the case of alternative model formulations, because it is perfectly possible to build models with different implementations producing similar results as there is not a unique mapping between a model and the system under study.
} 
fitness metric and should be seen as auxiliary tool for supporting the hypothesis that these specific model formulations differences should be considered as relevant.

\subsection{Sensitivity analysis of Model 3}

In this section we show and discuss the results of Sobol variance decomposition method which has been used for the global sensitivity analysis of the model type 3 output. As we have previously mentioned in this work, the model/strategy type 3 consists basically in one of the alternative formulations of the conjugation rule used in this study which takes into account the parameter $\gamma_{1}$ (cellCycle) for linking the conjugative event to the host cell cycle. The methodology employed for analyzing systematically the representativeness of every model parameter in the total variance of the output has been introduced previously in the Chapter 6 , Section 6.1.2, consisting in the estimation of both first and total order indices[STCR04][Sal08] for model output under study.

The model type 3 , according to the data which has been shown in the previous sections of this chapter as well as in the previous chapter, provides the best computational representation for the plasmid spread dynamics, when compared to the two alternative representations. The objective of performing this type of analysis is to understanding how the output variance should be apportioned to each model parameter in order of being able to handle consistently the uncertainty on the model inputs. Additionally, the results of this method may provide another evidence for supporting our claim about the importance of time-related aspects when building a discrete time conjugation model.

We have analyzed the model contrasting its outputs to the experimental dataset (The experimental data was kindly provide by the Professor Fernando de la Cruz laboratory) using the time series of conjugation rate from three different plasmids, namely the pAR118, the pSU2007 and a pR388(MOB) which are representative examples, respectively of a repressed system, a de-repressed one which constitutively express the conjugative machinery and finally the last plasmid is a non-conjugative construction. The pAR118 is a derivative of R1 plasmid and the last two are based on the pR388 backbone. In order to undertake the sensitivity analysis, we have defined an objective 
function for comparing the subset of model outputs for which the experimental data were available. Specifically, these outputs are the time-series for the conjugation rate $T /(T+R)$ and the doubling of both donors and transconjugant cells. The conjugation rate data was not used directly in the objective function, instead for simplifying the comparison between experimental and simulated data, an simple third degree polynomial equation was fitted to the reference data which are shown in the Table 7.2 presented previously in this manuscript.

Bearing in mind the reproducibility of the experimental results obtained from the application of Sobol method, all code required for running the same experiments are shown in the Appendix A.

It is important to remark that the results presented here are preliminary ones because it is not feasible, using the computational resources of a desktop computing, to perform the required number of model execution for reaching an stable output. According to the stability results, which have been previously shown in the Figure 6.6, we may realize that due to the individual variability the model require a minimum number of replications for the same parameter set in order to produce stable outputs5.

The computational cost of applying the Sobol method is $N \times(p+2)$ model evaluations, where $N$ is the number of samples and $p$ the number of input parameters being analyzed. Therefore, assuming a value of $N=1000$, the number of input factors are $p=6$ and the minimal number of experimental replications are $r=15$ we find that we need to execute the model at least $64 K$ times. This is a very high number of executions for a costly individual-based model which requires approximately 30 minutes for simulating 6 hours of bacterial growth using a grid size equal to $1000 \mu m^{2}$ hence, as the model results are stable (data no shown) with a reduced grid dimensions, we have chosen to use a grid size of $250 \mu \mathrm{m}^{2}$ for practical reasons.

Even using the reduced grid size, the above number of model executions are still prohibitive without the use of a large parallel cluster 6 . The complete sensitivity analysis for each experimental setup would take approximately 192 hours of wall time in a hardware based on Intel(R) Core(TM) i7-2630QM processor at $2.00 \mathrm{GHz}$ clock with

\footnotetext{
${ }^{5}$ It is required approximately 50 replications for each set of input factors for achieving a completely steady model output. But it can be also observed that with roughly 30 replications the model variability starts to converge very significantly. Finally, with replication values greater than 15 , the amplitude of the observed oscillations can be accepted as the minimal number of replications for getting stable results.

${ }^{6}$ We are planning to reassess the model distributing the workload across several machines
} 
$8 \mathrm{~GB}$ being the model execution parallelized across all available cores7. Additionally, the system resources become depleted due to the lack of resources devoted to the heap space of all virtual machines running in parallel. Thereby, for performing the model analysis we have had to sacrifice some precision in order to circumvent the these practical limitations. In the experiments shown in this chapter, we have had to reduce the number of samples, using a $N=500$ with one replications of each set of input factors.

In some cases, this setup have produced somewhat unreliable results, possibly due to the interactions between the model parameters and the large variability as consequence of the individual levels stochasticity. In these cases we have also analyzed the sensitivity analysis outputs but noting that certainly the experiment would require more replications in order to provide adequate outputs. The current simulation setup requires approximately 20 hours to complete.

\subsubsection{Sobol indices for a repressed plasmid}

In general the experiment for the repressed plasmid pAR118 has shown a some instability with respect to the input parameters producing indices with negative values, indicating that is necessary to increment the number of model samples in order to achieve a more stable results.

Figures 8.12, 8.13, 8.14 and 8.15 contains the first order indices and the total order indices calculated using the Sobol method of variance decomposition applied to simulation output of the repressed plasmid pAR118 using and initial donor to recipient ratio of $5 \%$ for the input factors conjugationCost, $\gamma_{0}$ and cyclePoint. Figure 8.12 shows the contribution of the input parameters on the conjugation rate expressed as the ratio $T /(T+R)$. The Figure 8.13 has the effect of the model input parameters on the generation time of donor cells. The Figure 8.14 shows the effect that every input parameter has on the generation time of transconjugant cells. Finally, the 8.15 contains the effects of the model input parameters on the composite output which is the sum of these three previous outputs.

As can be observed in the Figure 8.12 , where the Sobol indices for the conjugation

${ }^{7}$ The last version of R/Repast parallelize the individual model execution using the doSNOW package https : //CRAN. R-project . org/package=doSNOW 


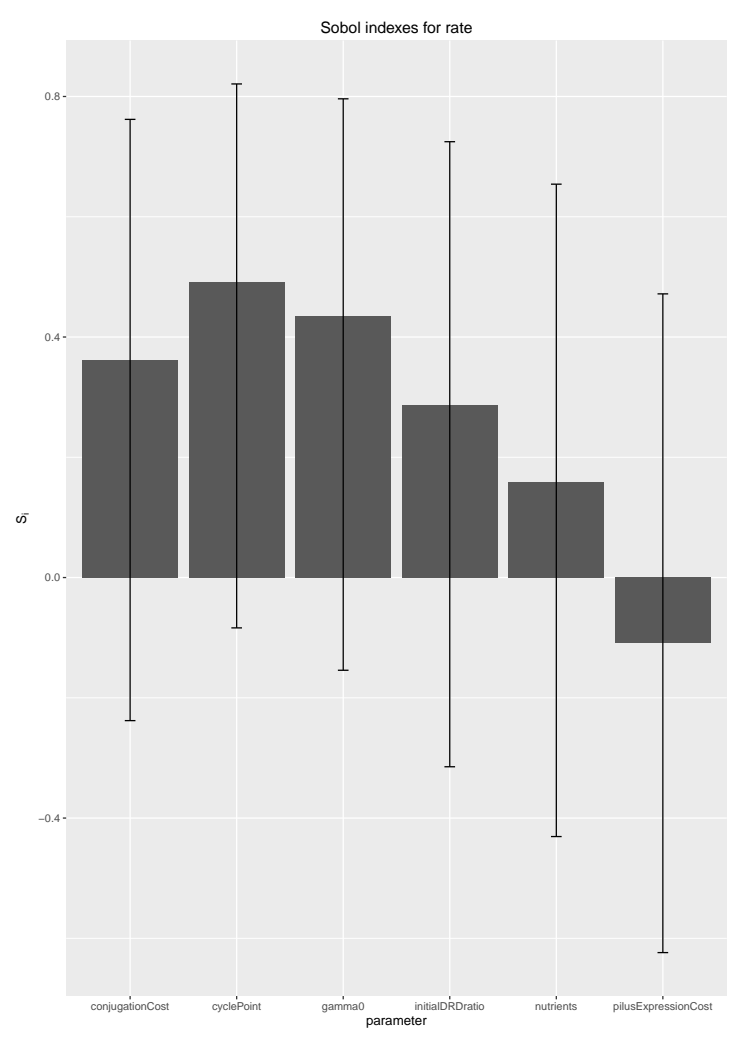

(a) First order

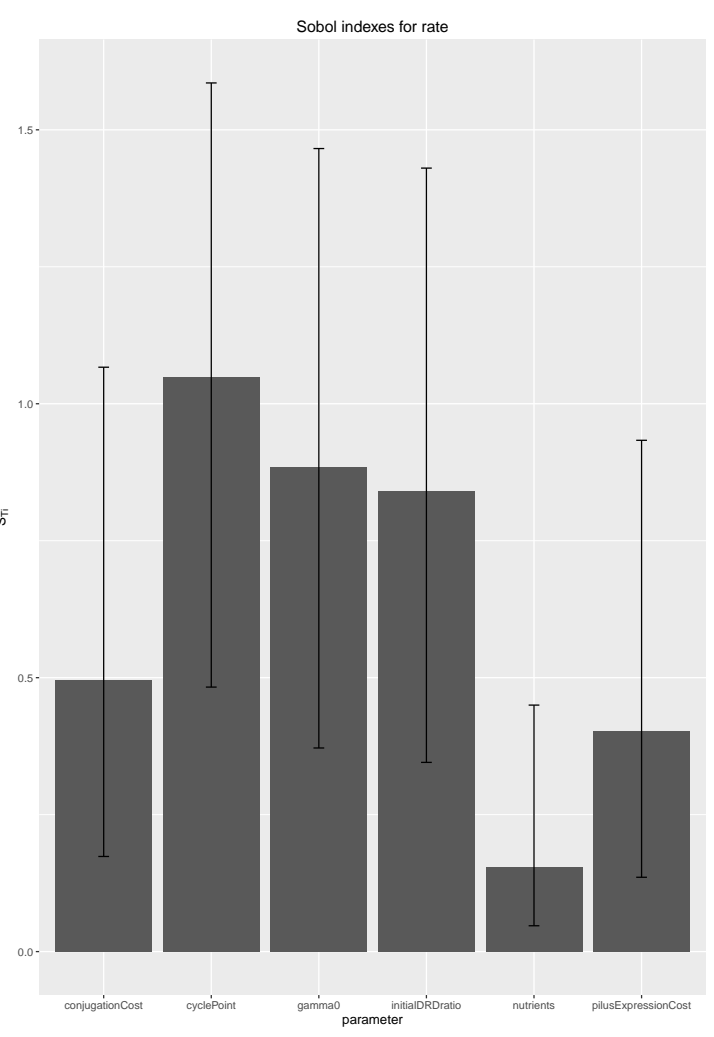

(b) Total order

Figure 8.12: Sensitivity analysis for the plasmid pAR118 using an initial donor density of 5\%. The first and total order Sobol sensitivity metrics, respectively the subfigures (a) and (b), for the BactoSIM model parameters with respect to the conjugation rate $T /(T+R)$ output.

rate $T /(T+R)$ are shown, both first and the total order indices are telling us that the most important parameter is the point in the cell cycle when conjugative transfer is most likely to happen, followed by the $\gamma_{0}$, the initial de-repressed donor ratio, the conjugation cost, the cost of expressing the T4SS conjugative pili and finally the nutrient availability. These results seems to be consistent which the expected model behavior and with the logic behind the conjugative process. The first order indices $S_{i}$ shows a negative value for pili expression cost which could be possibly attributed to need of increasing the number of samples but also due to the non-linear interactions between the model input factors.

The Sobol indices for the doubling time output of both donor and transconjugant 


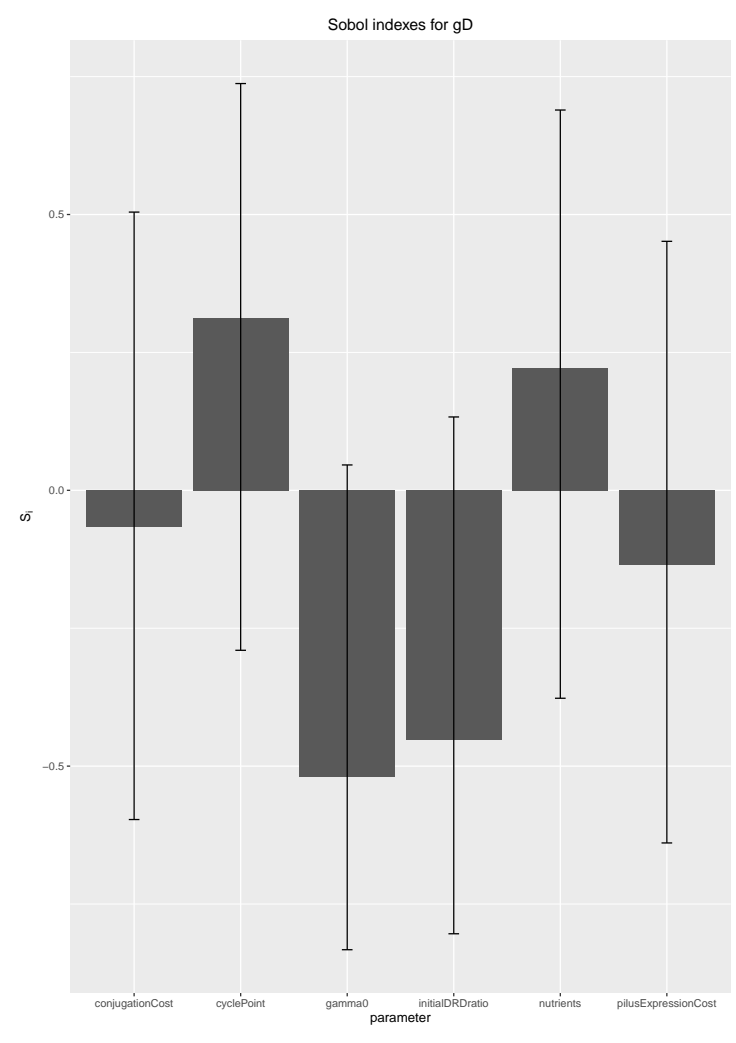

(a) First order

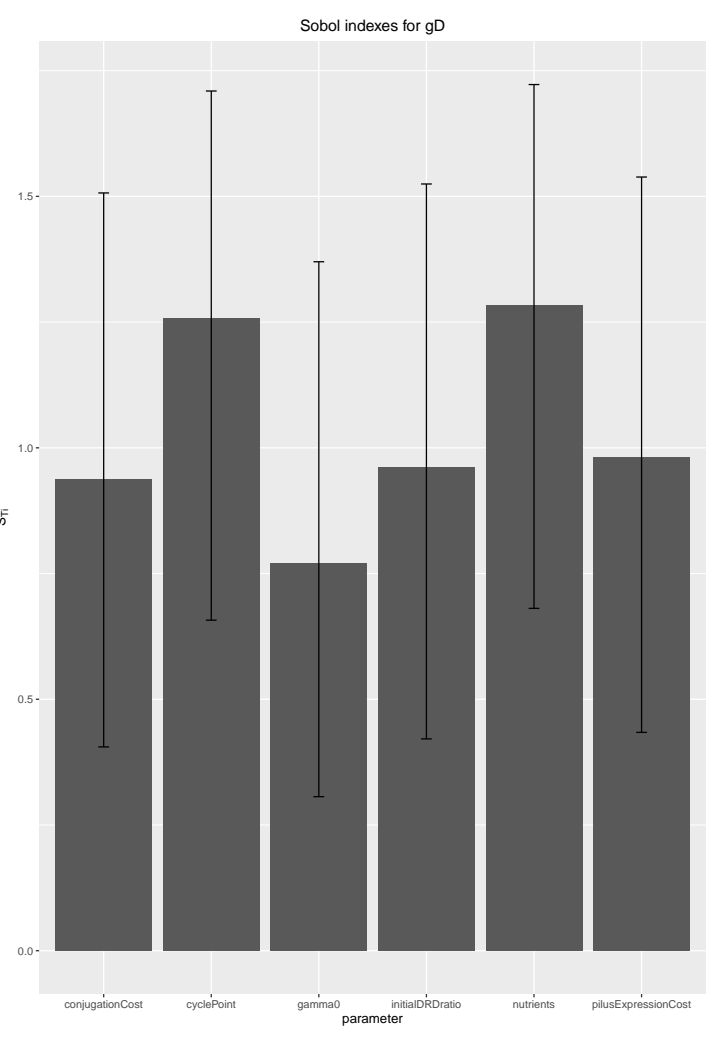

(b) Total order

Figure 8.13: Sensitivity analysis for the plasmid pAR118 using an initial donor density of 5\%. The first and total order Sobol sensitivity metrics, respectively the subfigures (a) and (b), for the BactoSIM model parameters with respect to the generation time of plasmid donor cells (D) output.

cells shown in Figures 8.13 and 8.14 have some noise and requires more samples for obtaining better results. The first order indices $S_{i}$ of both outputs present four negative values therefor we avoid to draw any conclusions about it. Nonetheless, the total order indices $S_{T i}$ shows for the doubling time of donors that the cost of conjugation and the expression of conjugative apparatus are equally important which is consequence of the fact that donors cells also require some expression level of T4SS genes. On the other hand the most significant input factor for transconjugant cells is the cost of T4SS expression. It is also worth to mention that the initial de-repressed donor has a high impact on the doubling time output of both donors and transconjugants.

Finally, the overall effect on the composite output is shown in Figure 8.13 which 


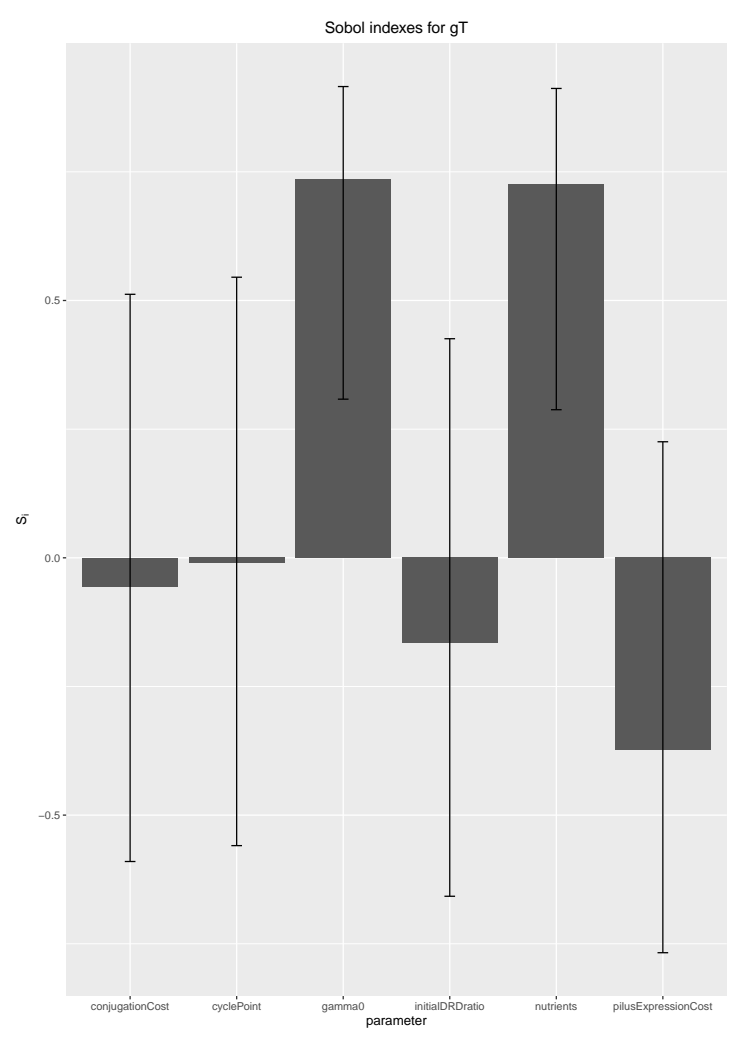

(a) First order

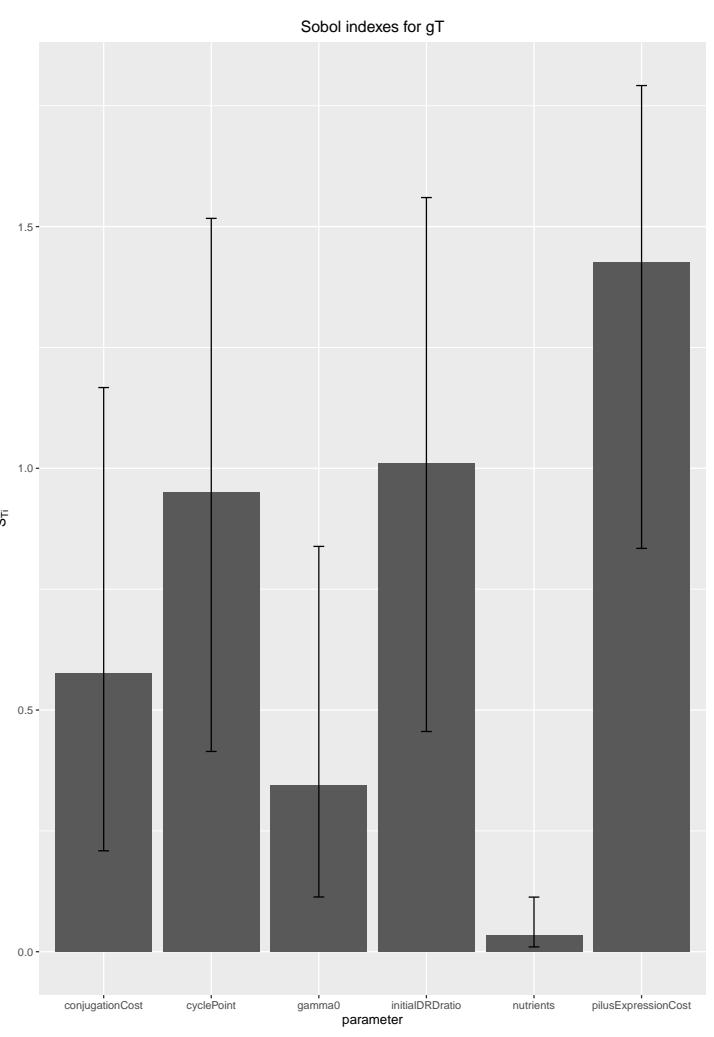

(b) Total order

Figure 8.14: Sensitivity analysis for the plasmid pAR118 using an initial donor density of 5\%. The first and total order Sobol sensitivity metrics, respectively the subfigures (a) and (b), for the BactoSIM model parameters with respect to the generation time of transconjugant cells (T) output.

is noise but seems to confirm the global significance for the cost of expressing the conjugative machinery, followed by the cycle point and the de-repressed donor ratio.

Figures 8.16, 8.17, 8.17 and 8.19 contains the first order indices and the total order indices calculated using the Sobol method of variance decomposition applied to simulation output of the repressed plasmid pAR118 using and initial donor to recipient ratio of $50 \%$. The Figure 8.16 shows the contribution of the input parameters on the conjugation rate expressed as the ratio $T /(T+R)$. The Figure 8.17 has the effect of the model input parameters on the generation time of donor cells. The Figure 8.18 shows the effect that every input parameter has on the generation time of transconjugant cells. Finally, the 8.19 contains the effects of the model input parameters on the composite 


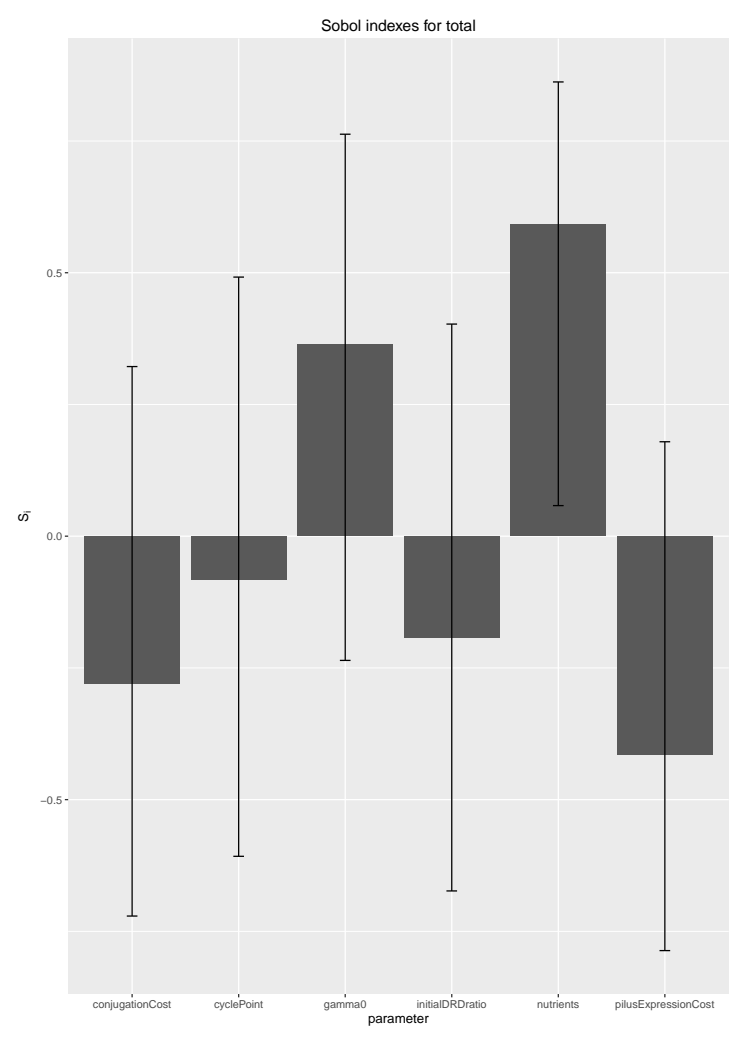

(a) First order

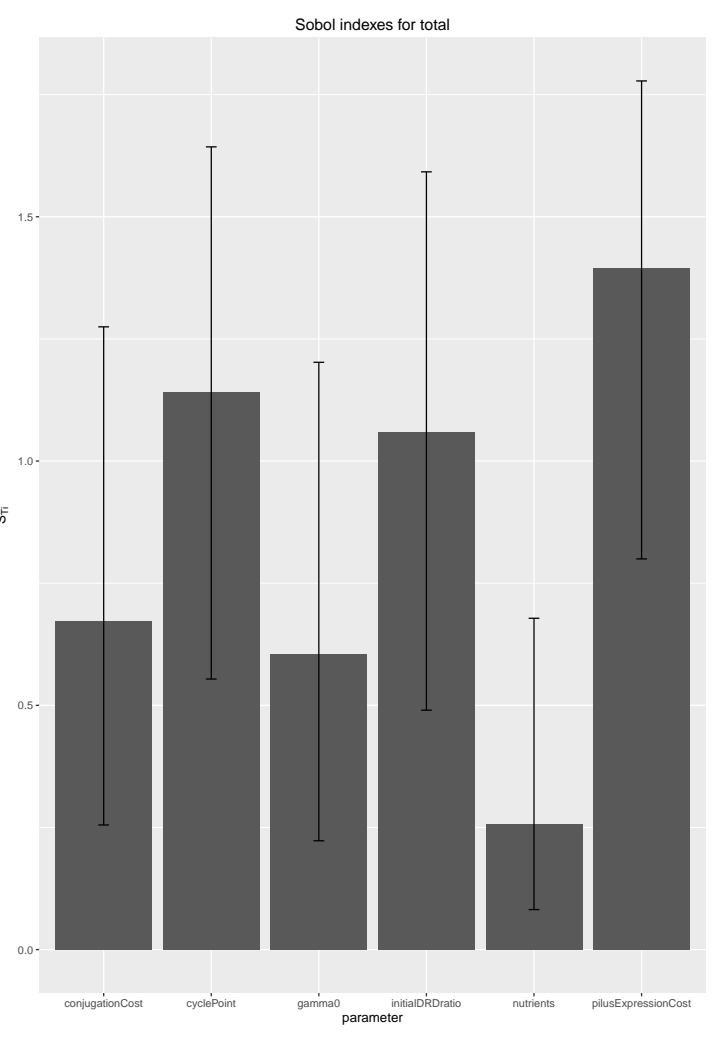

(b) Total order

Figure 8.15: Sensitivity analysis for the plasmid pAR118 using an initial donor density of 5\%. The first and total order Sobol sensitivity metrics, respectively the subfigures (a) and (b), for the BactoSIM model parameters with respect to the composite effect output which is the sum of individual effects over $T /(T+R)$ plus the effects on the generation time of donors and transconjugant cells.

output which is the sum of these three previous outputs.

\subsubsection{Sobol indices for a de-repressed plasmid}

Figures 8.20, 8.21, 8.21 and 8.23 contains the first order indices and the total order indices calculated using the Sobol method of variance decomposition applied to simulation output of the repressed plasmid pSU2007 using and initial donor to recipient ratio of $5 \%$. The Figure 8.20 shows the contribution of the input parameters on the conjugation rate expressed as the ratio $T /(T+R)$. The Figure 8.21 has the effect of the model input parameters on the generation time of donor cells. The Figure 8.22 shows 


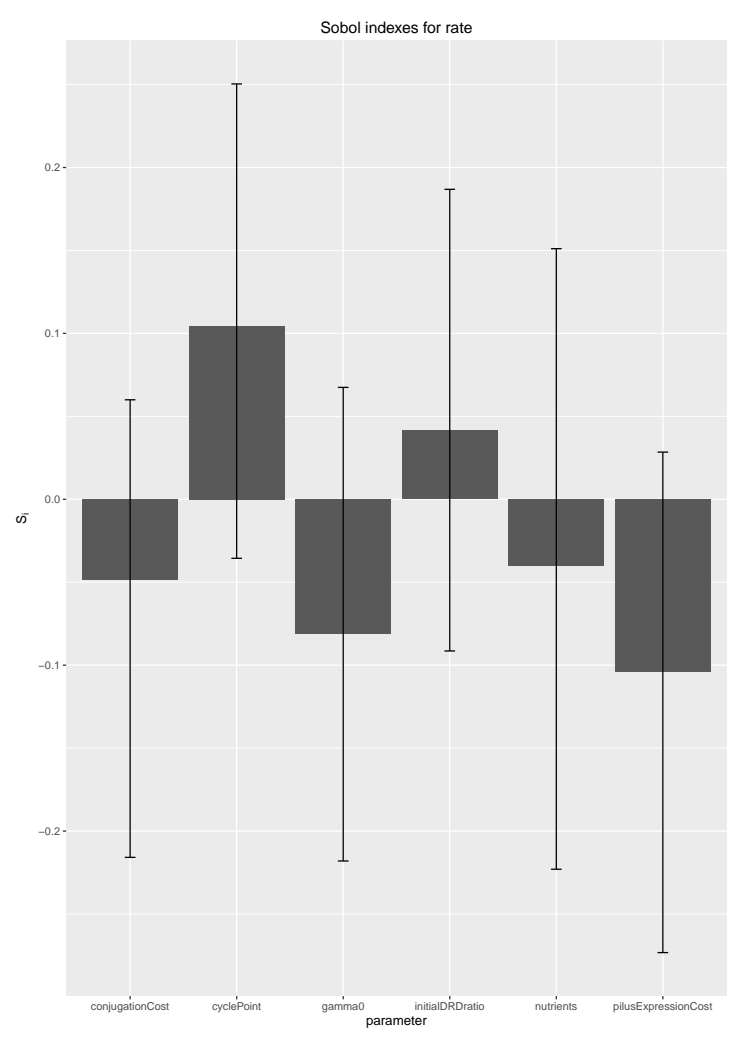

(a) First order

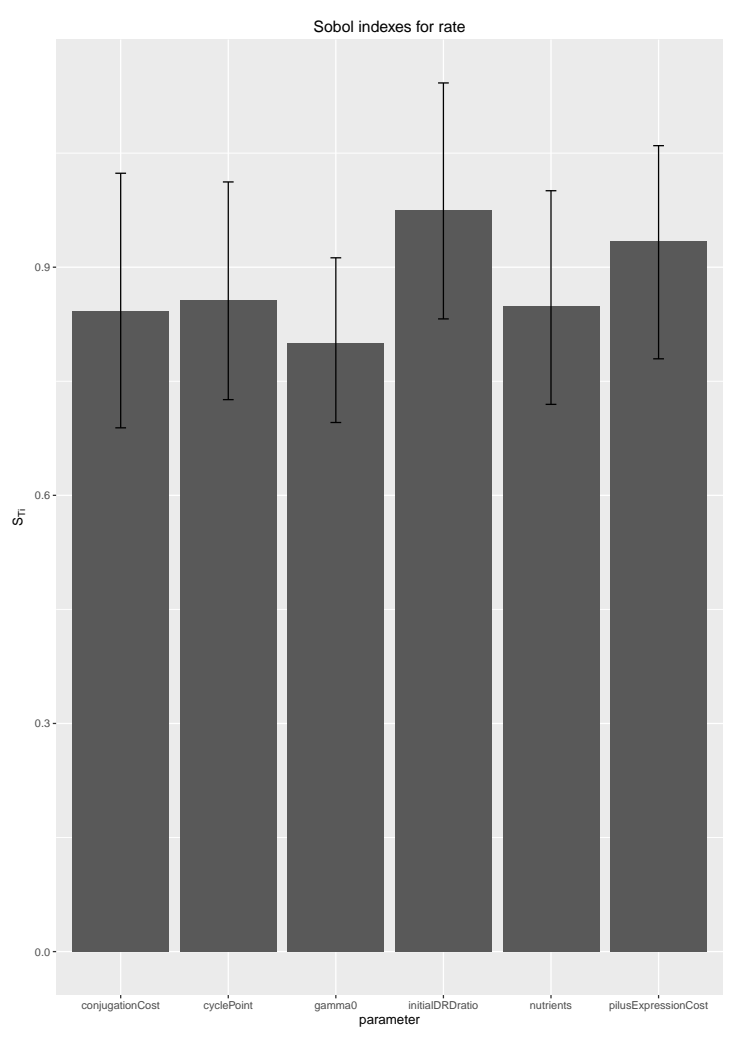

(b) Total order

Figure 8.16: Sensitivity analysis for the plasmid pAR118 using an initial donor density of 50\%. The first and total order Sobol sensitivity metrics, respectively the subfigures (a) and (b), for the BactoSIM model parameters with respect to the conjugation rate $T /(T+R)$ output.

the effect that every input parameter has on the generation time of transconjugant cells. Finally, the 8.23 contains the effects of the model input parameters on the composite output which is the sum of these three previous outputs.

The sensitivity analysis for the de-repressed plasmid pSU2007 with an initial donor density of $5 \%$ shows a strong influence of the nutrient availability which clearly dominates over the remaining input factors as can be observed for the first and total order indices $S_{i}$ and $S_{T} i$ shown in the Figure 8.20. The next parameters in order of importance are the cycle point and $\gamma_{0}$. Finally, it can be also observed from the total order plot, that cost of T4SS expression followed by the conjugation costs are the last ones contributing in the total variance. 


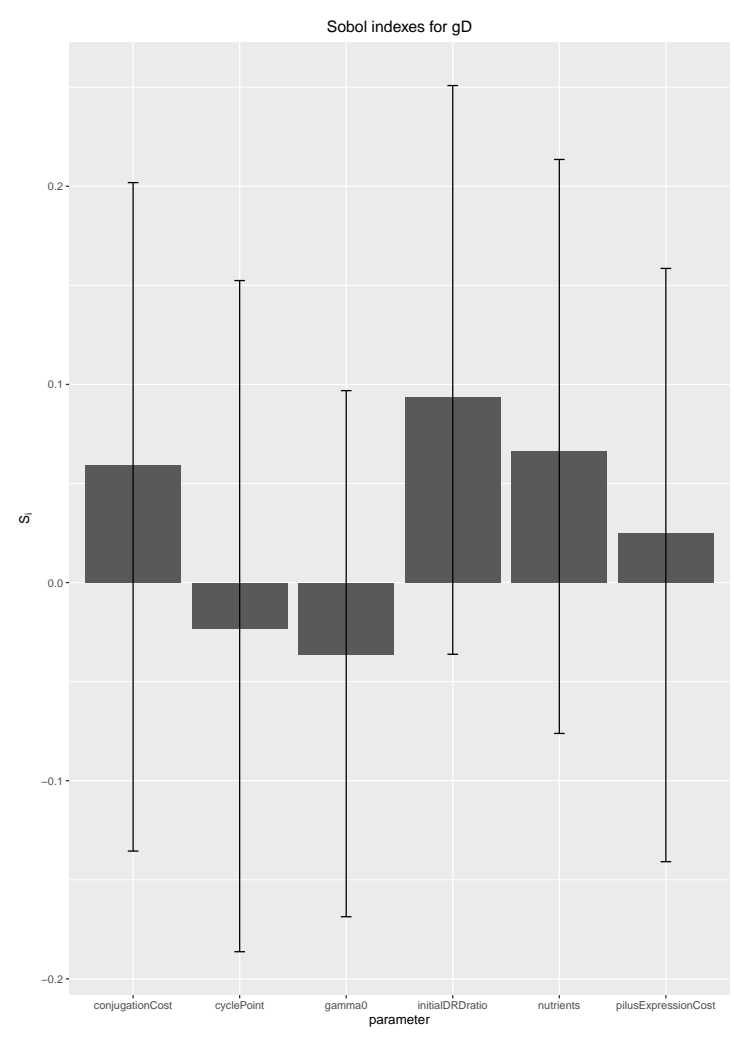

(a) First order

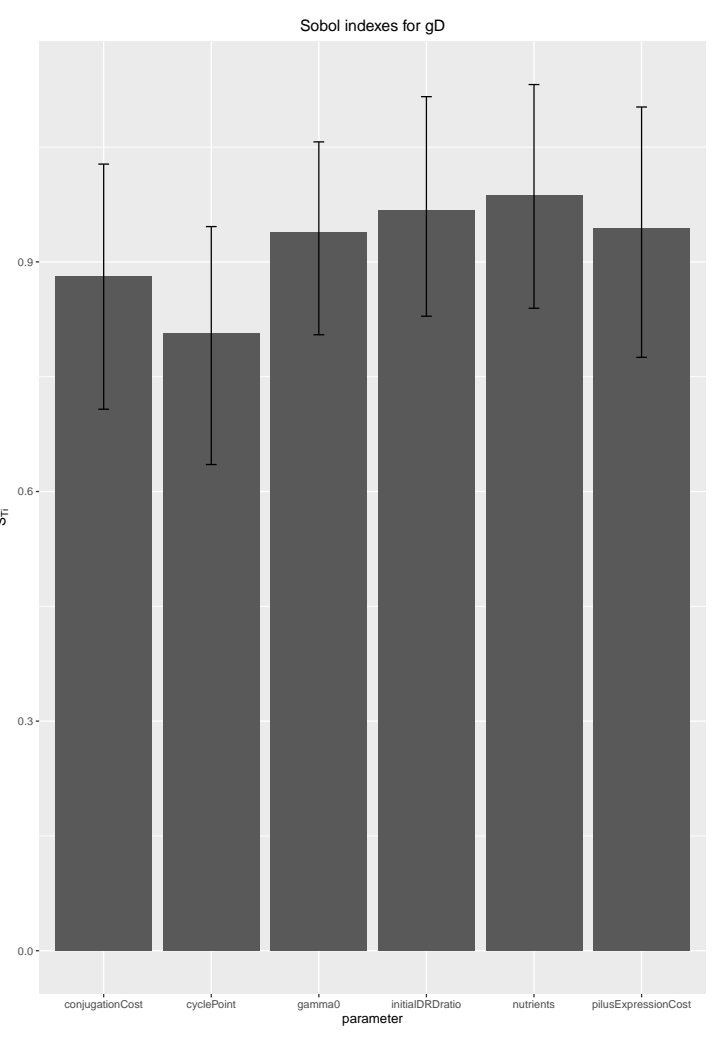

(b) Total order

Figure 8.17: Sensitivity analysis for the plasmid pAR118 using an initial donor density of $50 \%$. The first and total order Sobol sensitivity metrics, respectively the subfigures (a) and (b), for the BactoSIM model parameters with respect to the generation time of plasmid donor cells (D) output.

The results of the sensitivity analysis for output variable representing the doubling time of donors cells which are presented in Figure 8.21. Both first order and total order indices are clearly pointing that variability of donor generation time is practically controlled by the cost of expressing the conjugative pili and followed at some distance by the conjugationCost. The other model parameters seems to present some nonlinear interaction with respect to this output. This results seems to be consistent with the common senses that the most costly part of the donor metabolic effort is due to the expression of conjugative apparatus.

In the case of transconjugant cells the Sobol indices shown in Figure 8.22 are saying practically the same as in the previous case, that is to say, the generation time 


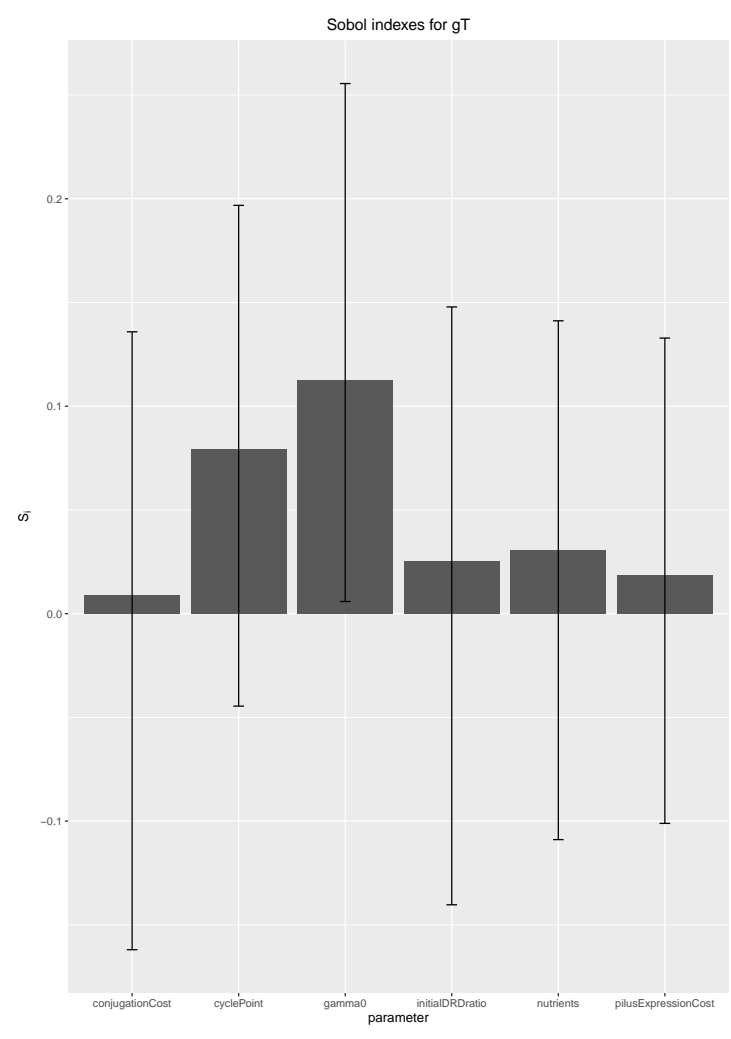

(a) First order

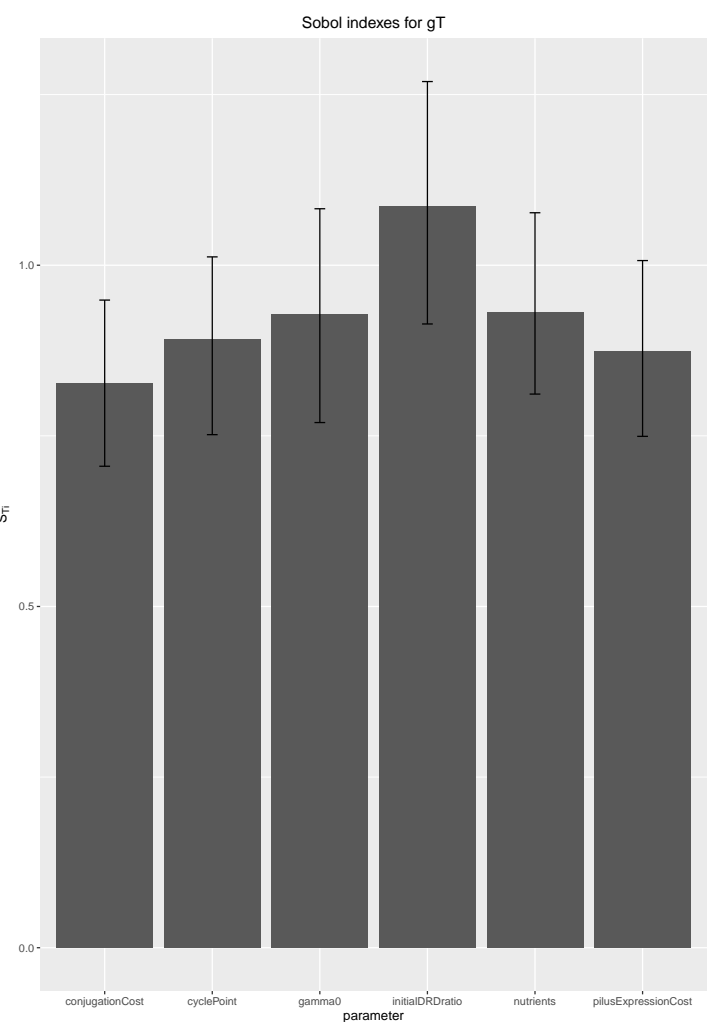

(b) Total order

Figure 8.18: Sensitivity analysis for the plasmid pAR118 using an initial donor density of 50\%. The first and total order Sobol sensitivity metrics, respectively the subfigures (a) and (b), for the BactoSIM model parameters with respect to the generation time of transconjugant cells (T) output.

is strongly coupled to the metabolic effort dedicated to express the T4SS genes with the remaining parameters showing non-additive interactions.

Finally, the composite metric shown in Figure 8.23 also indicates the greatest influence of the pilusExpressionCost in the overall model behavior and the remaining input factors seems to interact which is somehow expected due to the coupling between the other model parameters.

Figures 8.24, 8.25, 8.25 and 8.27 contains the first order indices and the total order indices calculated using the Sobol method of variance decomposition applied to simulation output of the repressed plasmid pSU2007 using and initial donor to recipient ratio of $50 \%$. The Figure 8.24 shows the contribution of the input parameters on the 


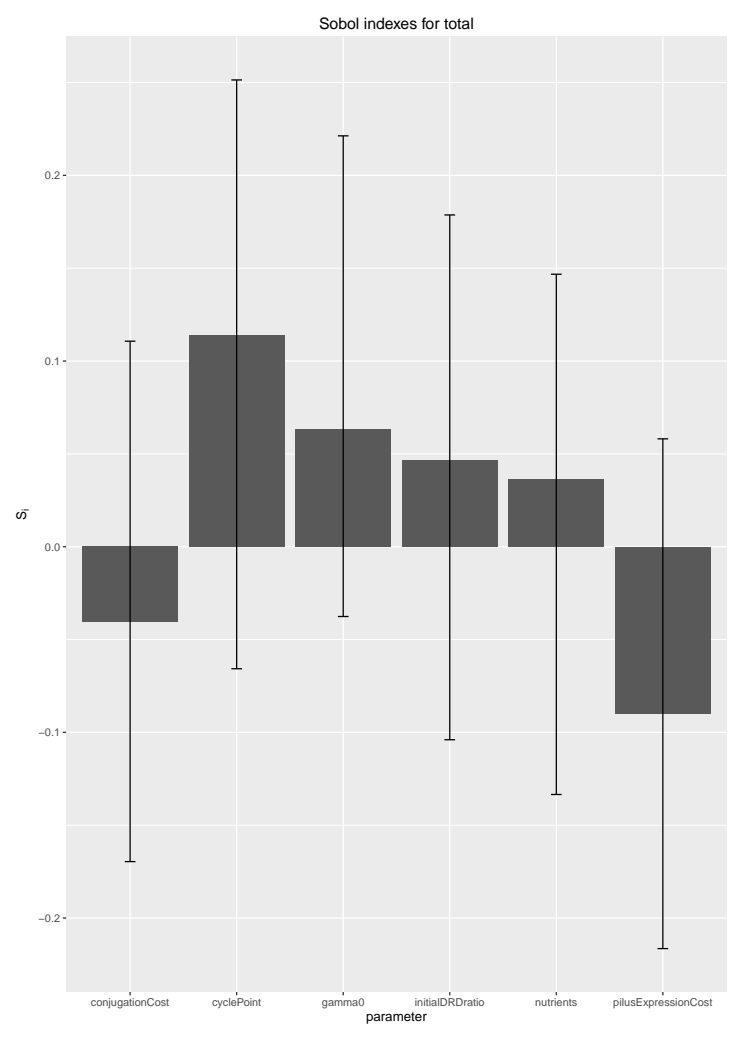

(a) First order

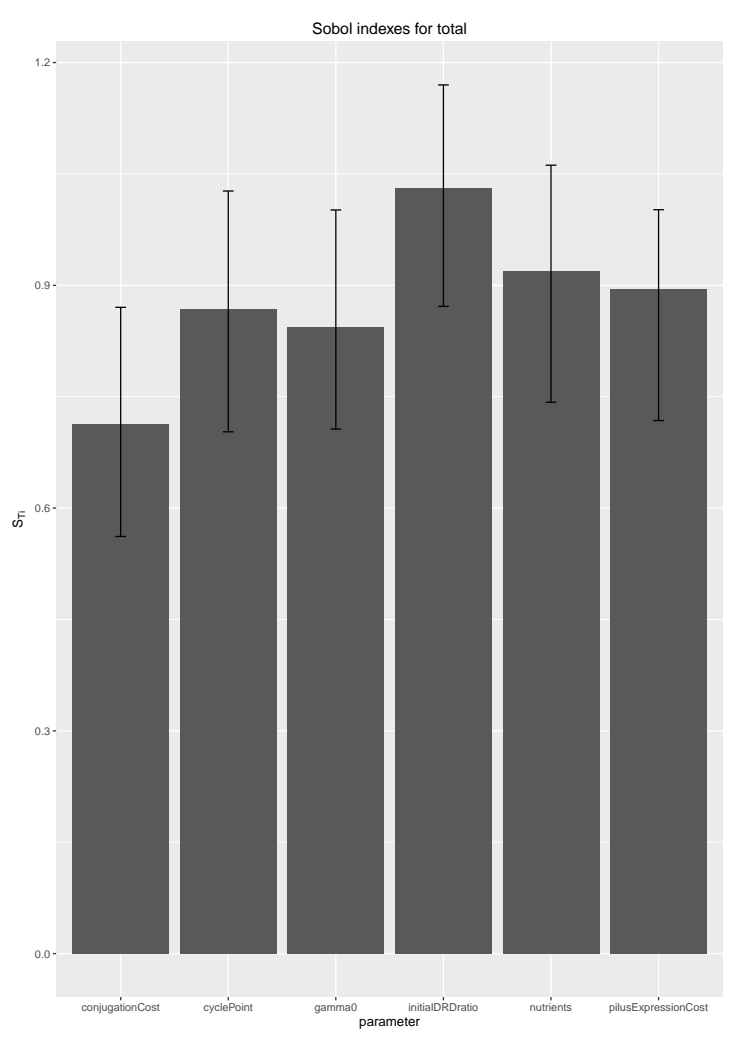

(b) Total order

Figure 8.19: Sensitivity analysis for the plasmid pAR118 using an initial donor density of $50 \%$. The first and total order Sobol sensitivity metrics, respectively the subfigures (a) and (b), for the BactoSIM model parameters with respect to the composite effect output which is the sum of individual effects over $T /(T+R)$ plus the effects on the generation time of donors and transconjugant cells.

conjugation rate expressed as the ratio $T /(T+R)$. The Figure 8.25 has the effect of the model input parameters on the generation time of donor cells. The Figure 8.26 shows the effect that every input parameter has on the generation time of transconjugant cells. Finally, the 8.27 contains the effects of the model input parameters on the composite output which is the sum of these three previous outputs.

Similarly as previously described in this section for the experiment with an initial donor density of $5 \%$, the sensitivity indices for pSU2007 using an initial concentration $50 \%$ presented in Figure 8.24 shows the importance of the model parameter related to the availability of substrate, followed by the cyclePoint, the $\gamma_{0}$, the expression cost of T4SS genes and finally the conjugation cost itself. Both first order and total order in- 


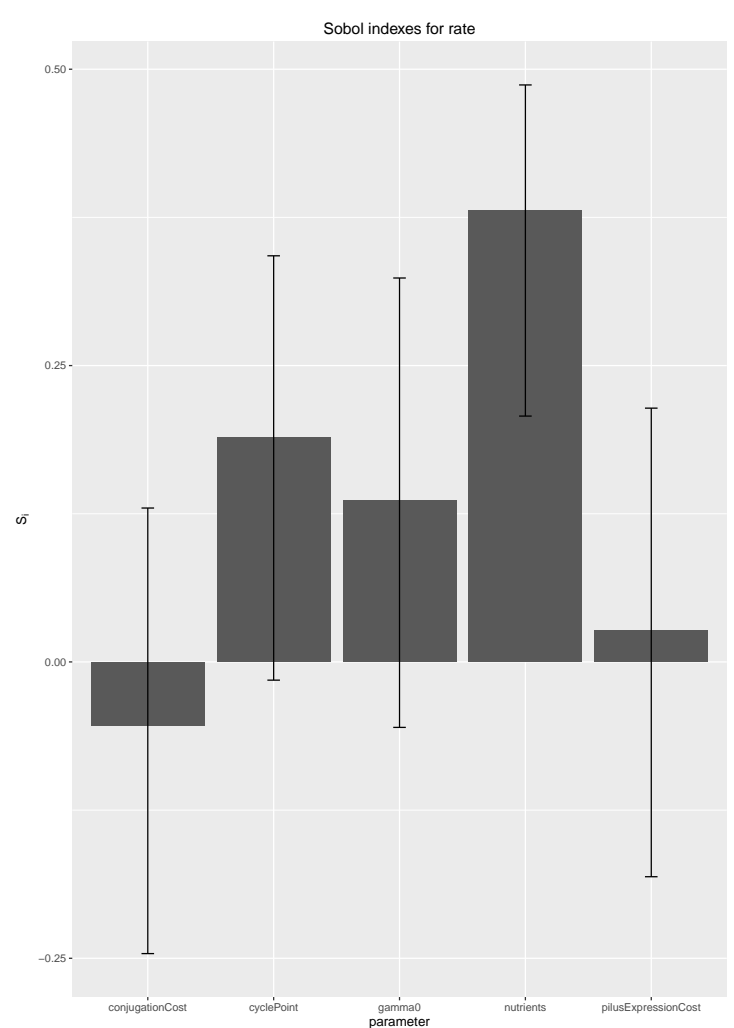

(a) First order

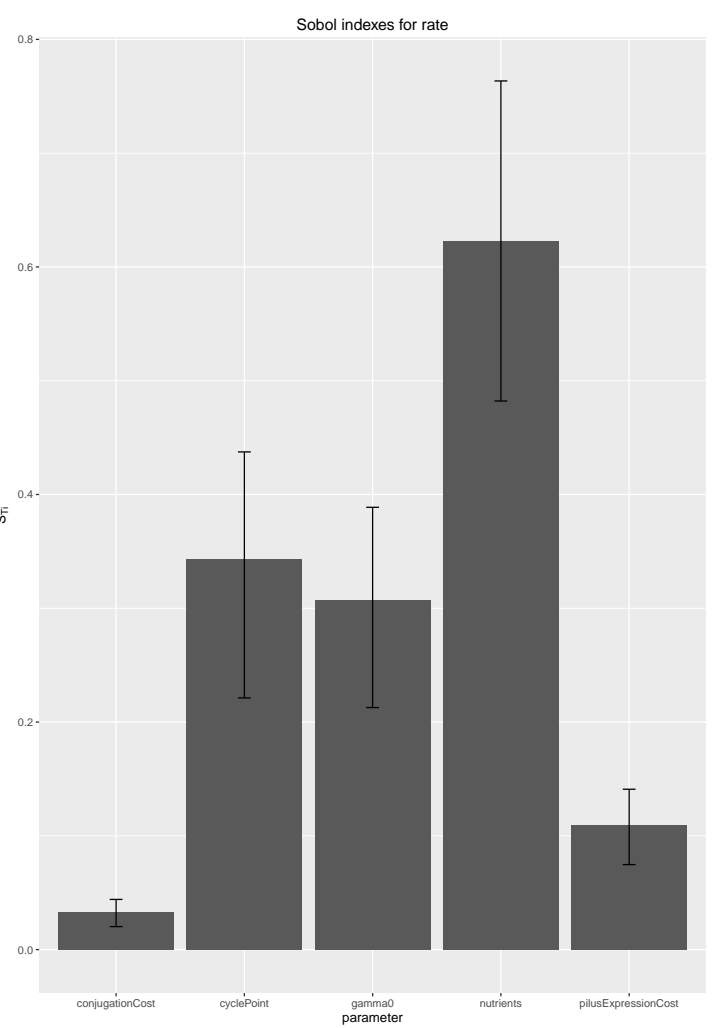

(b) Total order

Figure 8.20: Sensitivity analysis for the plasmid pSU2007 using an initial donor density of 5\%. The first and total order Sobol sensitivity metrics, respectively the subfigures (a) and (b), for the BactoSIM model parameters with respect to the conjugation rate $T /(T+R)$ output.

dices shows practically the same thing, differing from the cases of repressed plasmids where it seems to be more non-linear interactions between the model parameters.

With respect to the doubling time, the Sobol indices shown in Figure 8.25 says that the most part of the output variability of this variable come from the cost of expressing the conjugative machinery followed by the cost of conjugation but with much less importance than the first. The remaining parameters have similar impact on the output.

The sensitivity analysis for the doubling time of transconjugant cells, which are presented in Figure 8.26, also indicates a strong influence of the pilusExpressionConst in the variance of this model output followed by the nutrient availability. The remaining 


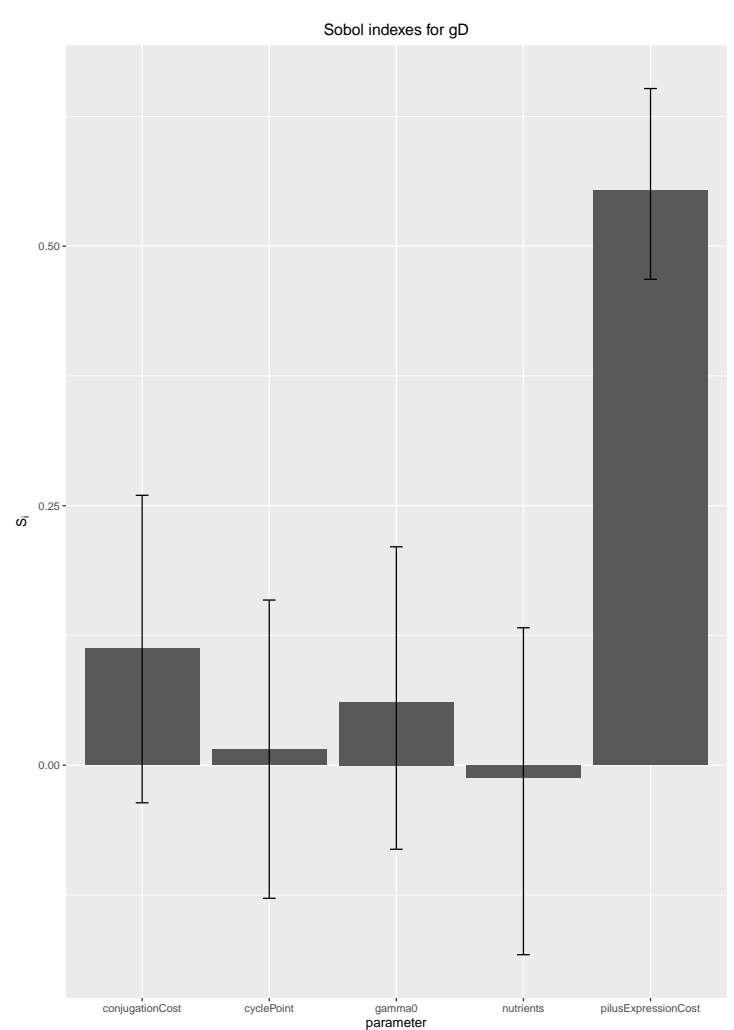

(a) First order

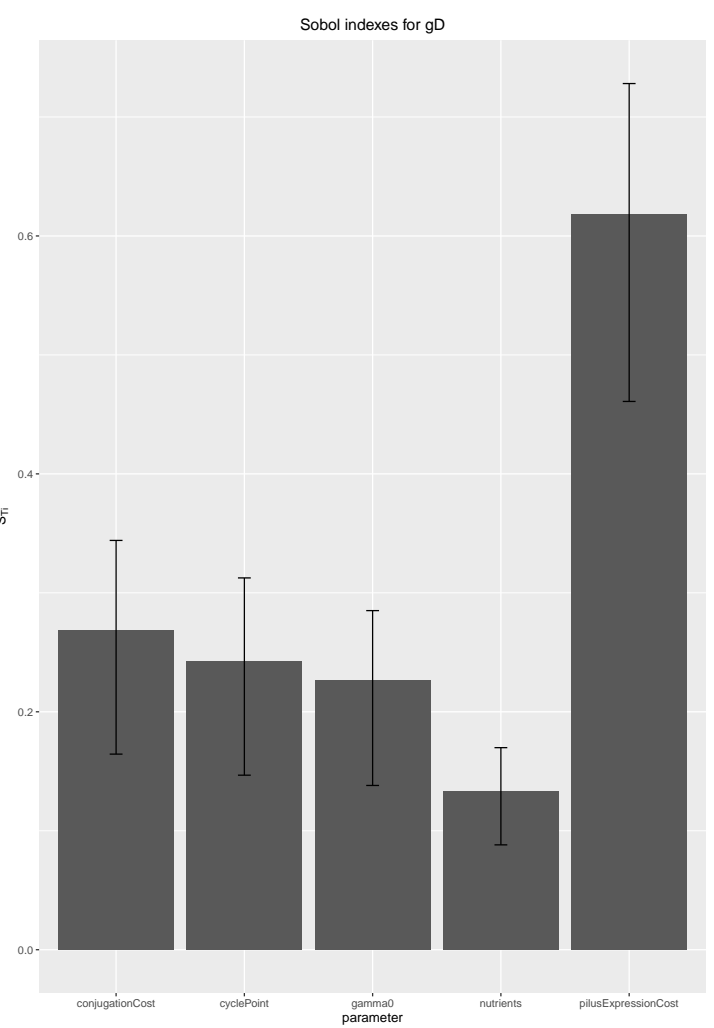

(b) Total order

Figure 8.21: Sensitivity analysis for the plasmid pSU2007 using an initial donor density of 5\%. The first and total order Sobol sensitivity metrics, respectively the subfigures (a) and (b), for the BactoSIM model parameters with respect to the generation time of plasmid donor cells (D) output.

parameters are much less influential on the output variable.

Finally, the composite output variable, shown in Figure 8.27, reproduces the pattern observed for the doubling time of the transconjugant cells. This basically means that the cost of expressing the conjugative apparatus has a very strong influence on the output, taking into account that the output defined here is a distance metric between the observed and the simulated data.

\subsubsection{Sobol indices for a mobilizable plasmid}

The mobilizable plasmid analyzed here in this section is a construction which uses the structural backbone of an R388 plasmid eliminating the OriT which is added to another 


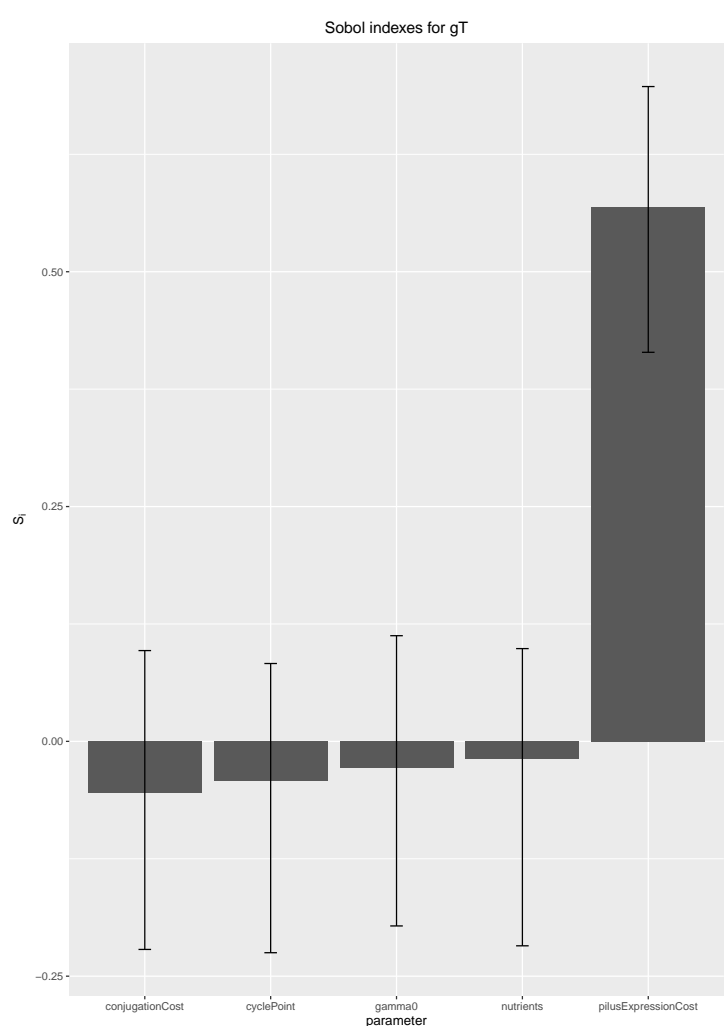

(a) First order

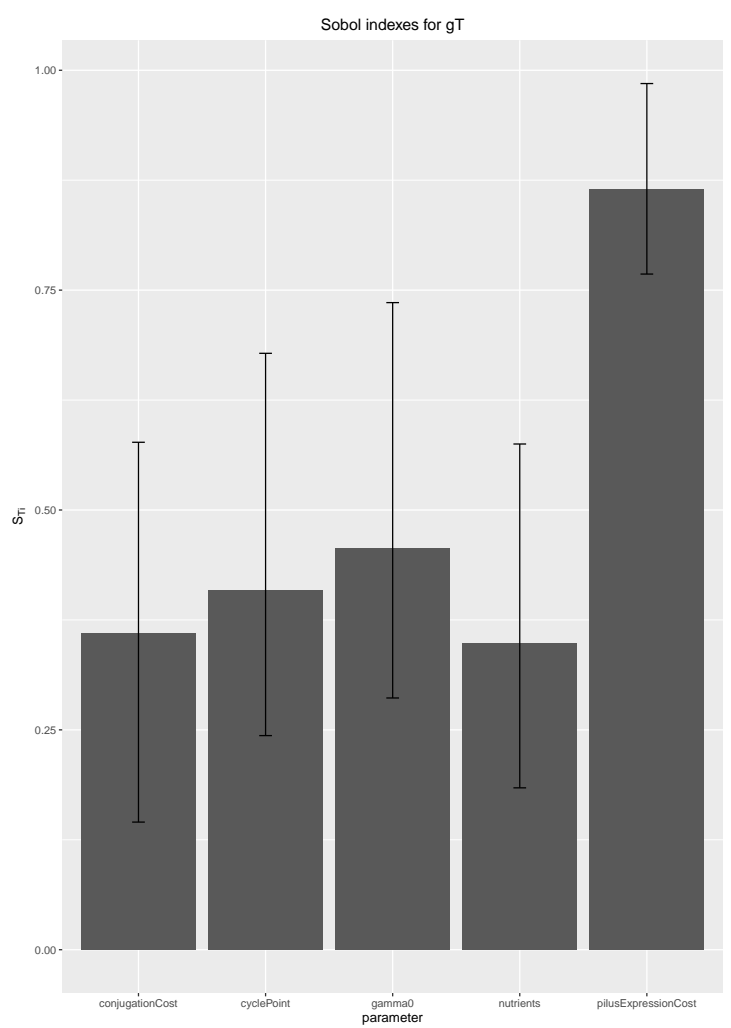

(b) Total order

Figure 8.22: Sensitivity analysis for the plasmid pSU2007 using an initial donor density of 5\%. The first and total order Sobol sensitivity metrics, respectively the subfigures (a) and (b), for the BactoSIM model parameters with respect to the generation time of transconjugant cells (T) output.

plasmid making it mobilizable when in presence of the helper R388 plasmid which contains the core genes required for expressing the conjugative machinery. Being more specific, the mobilizable element is an instance of the pGP12[SHL] plasmid containing the OriT of an R388 plasmid cloned upstream of an GFP reporter protein which is repressed in presence of R388. The helper plasmid is an R388 plasmid without a functional OriT and therefore, the plasmid itself cannot be transferred to recipient cells. Hence, the donor cells will contain both mobilizable and helper plasmids for transferring the mobilizable one but, consequently the transconjugant cells will not be able to engage in a second round of conjugative transfer [ $\left.\mathrm{dCRC}^{+12}\right]$.

Figures 8.28, 8.29, 8.29 and 8.31 contains the first order indices and the total 


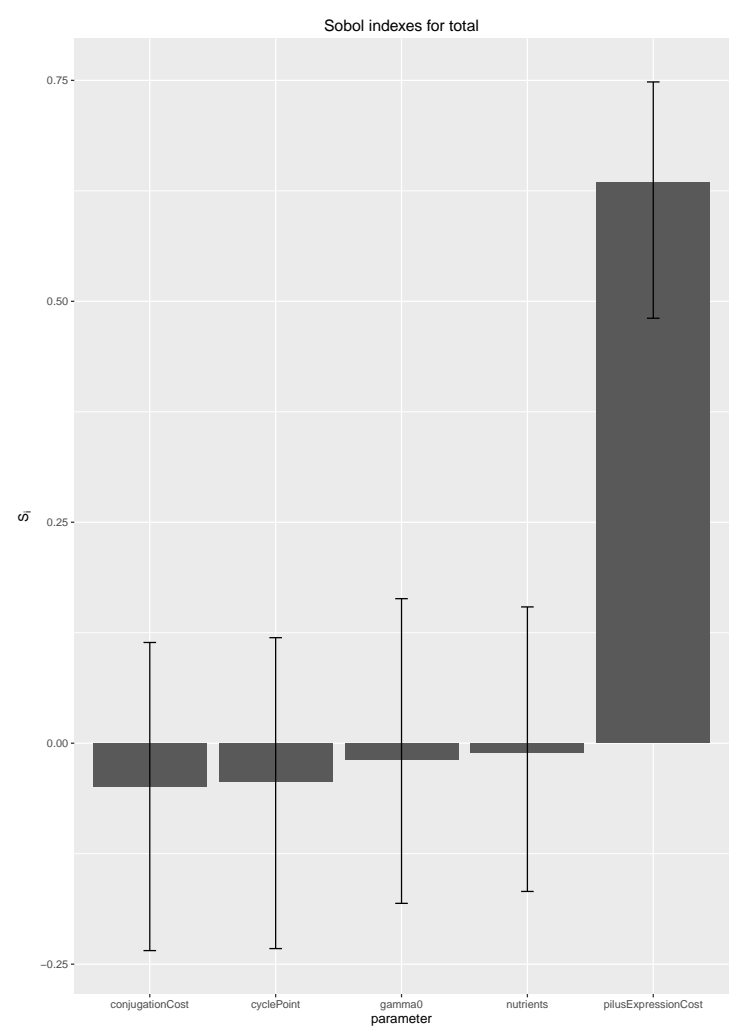

(a) First order

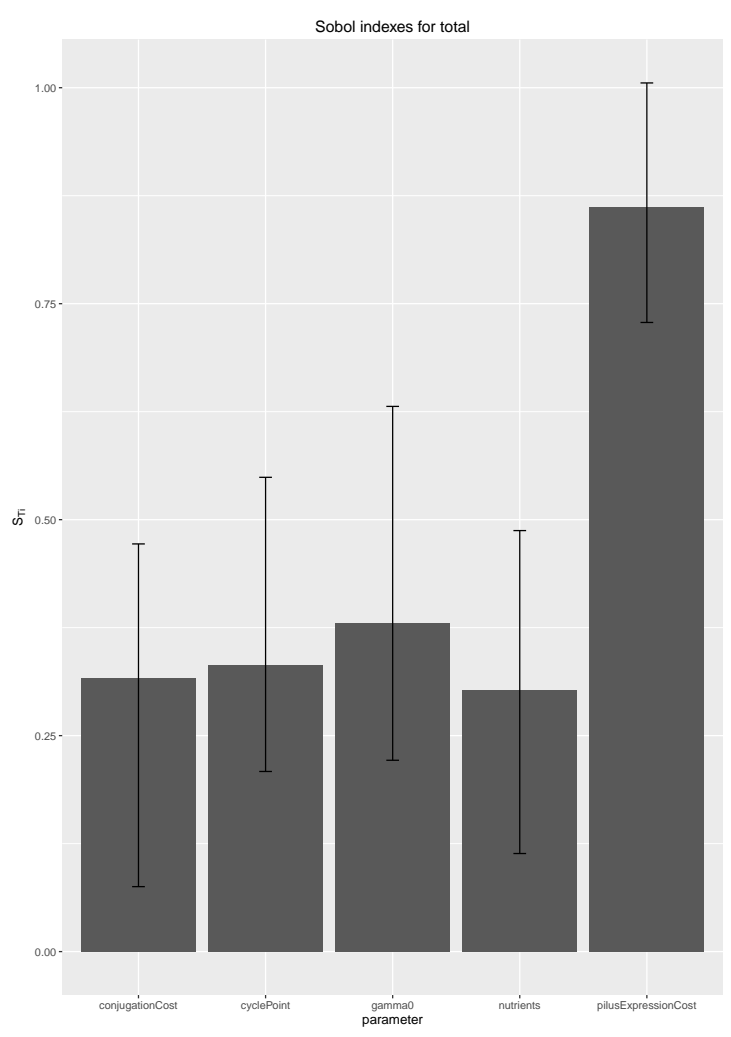

(b) Total order

Figure 8.23: Sensitivity analysis for the plasmid pSU2007 using an initial donor density of 5\%. The first and total order Sobol sensitivity metrics, respectively the subfigures (a) and (b), for the BactoSIM model parameters with respect to the composite effect output which is the sum of individual effects over $T /(T+R)$ plus the effects on the generation time of donors and transconjugant cells.

order indices calculated using the Sobol method of variance decomposition applied to simulation output of the repressed plasmid pR388(OriT) using and initial donor to recipient ratio of $5 \%$. The Figure 8.28 shows the contribution of the input parameters on the conjugation rate expressed as the ratio $T /(T+R)$. The Figure 8.29 has the effect of the model input parameters on the generation time of donor cells. The Figure 8.30 shows the effect that every input parameter has on the generation time of transconjugant cells. Finally, the 8.31 contains the effects of the model input parameters on the composite output which is the sum of these three previous outputs.

Figures 8.32, 8.33, 8.33 and 8.35 contains the first order indices and the total order indices calculated using the Sobol method of variance decomposition applied to 


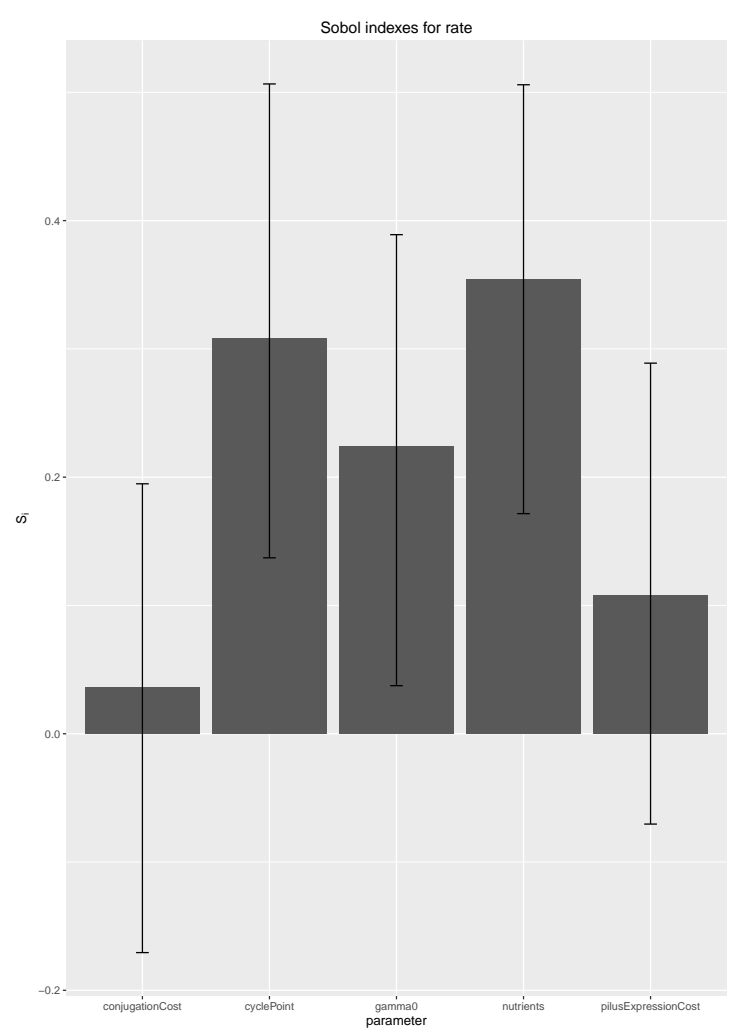

(a) First order

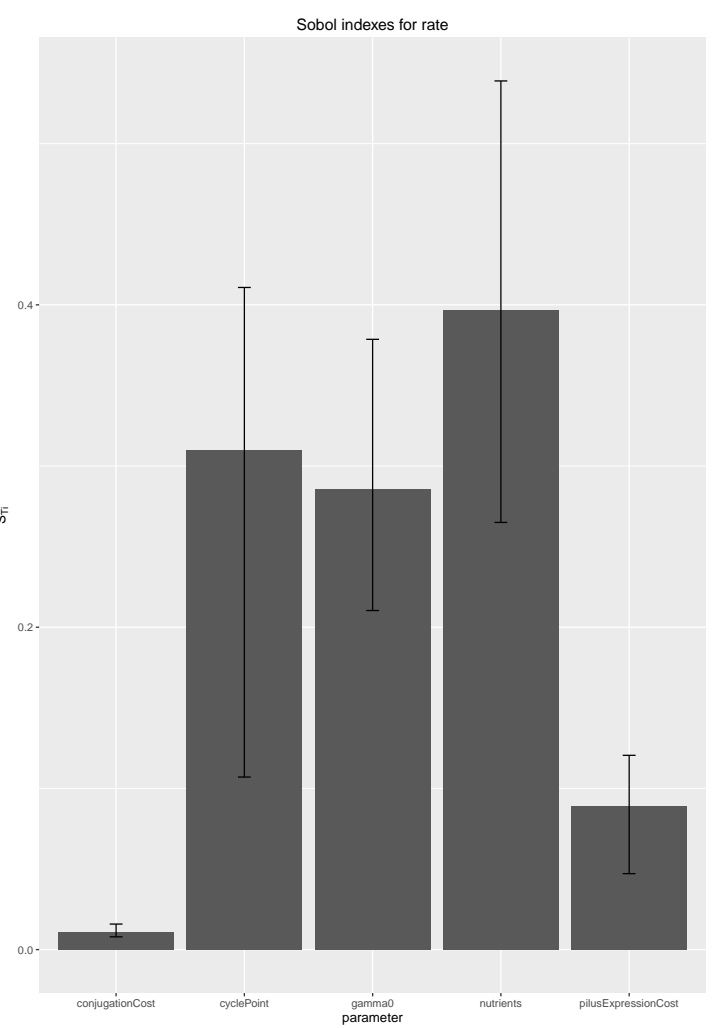

(b) Total order

Figure 8.24: Sensitivity analysis for the plasmid pSU2007 using an initial donor density of $50 \%$. The first and total order Sobol sensitivity metrics, respectively the subfigures (a) and (b), for the BactoSIM model parameters with respect to the conjugation rate $T /(T+R)$ output.

simulation output of the repressed plasmid pR388(OriT) using and initial donor to recipient ratio of $50 \%$. The Figure 8.32 shows the contribution of the input parameters on the conjugation rate expressed as the ratio $T /(T+R)$. The Figure 8.33 has the effect of the model input parameters on the generation time of donor cells. The Figure 8.34 shows the effect that every input parameter has on the generation time of transconjugant cells. Finally, the 8.35 contains the effects of the model input parameters on the composite output which is the sum of these three previous outputs.

The first order and total order Sobol indices for the conjugation rate $T /(T+R)$ output obtained from the simulation of a mobilizable plasmid termed R388(OriT) at an initial donor density of $50 \%$ presented in the Figure 8.32 , shows that the input 


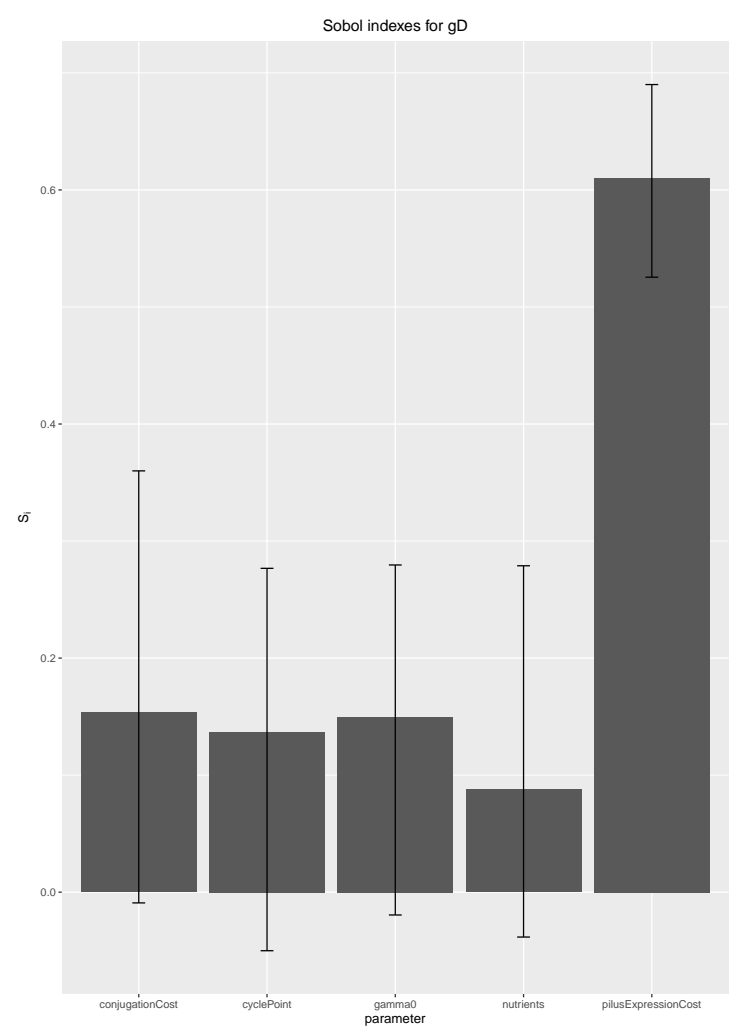

(a) First order

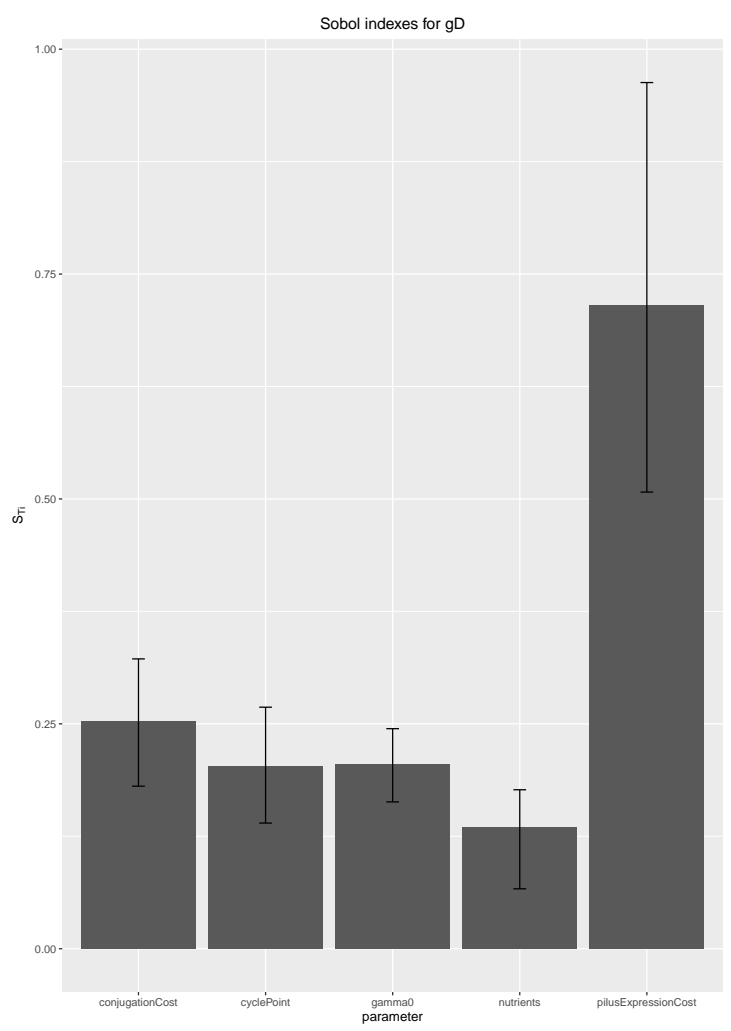

(b) Total order

Figure 8.25: Sensitivity analysis for the plasmid pSU2007 using an initial donor density of $50 \%$. The first and total order Sobol sensitivity metrics, respectively the subfigures (a) and (b), for the BactoSIM model parameters with respect to the generation time of plasmid donor cells (D) output.

factor cyclePoint clearly dominates over the remaining model parameters followed by the nutrient availability, the $\gamma_{0}$ and finally the both the conjugationCost and the pilusExpressionCost having similar importance.

The sensitivity analysis for the generation time of donor cells shown in Figure 8.33 indicates that the most import parameter is the expression cost of the T4SS conjugative machinery, which is approximately two times greater than the second in importance which is the cost of conjugation. The other parameters accounting for model variability are the cycle point, the $\gamma_{0}$ and the nutrient availability which apparently tends to interact non-additively as cab be seen in the discrepancies in the $S_{i}$ and $S_{T} i$. 


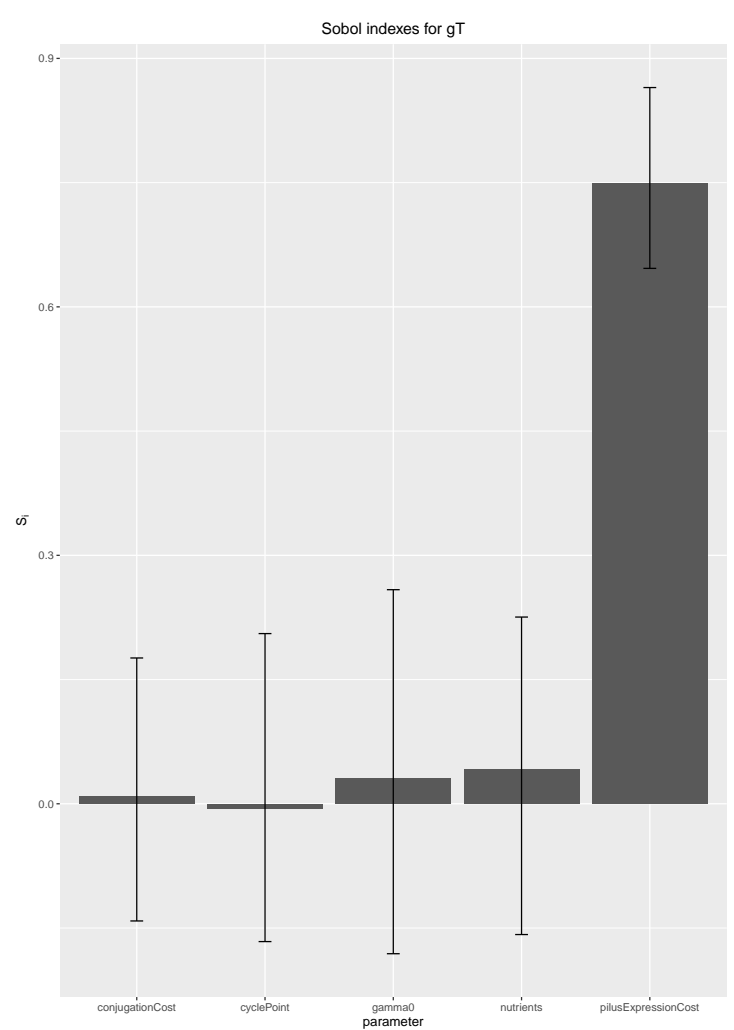

(a) First order

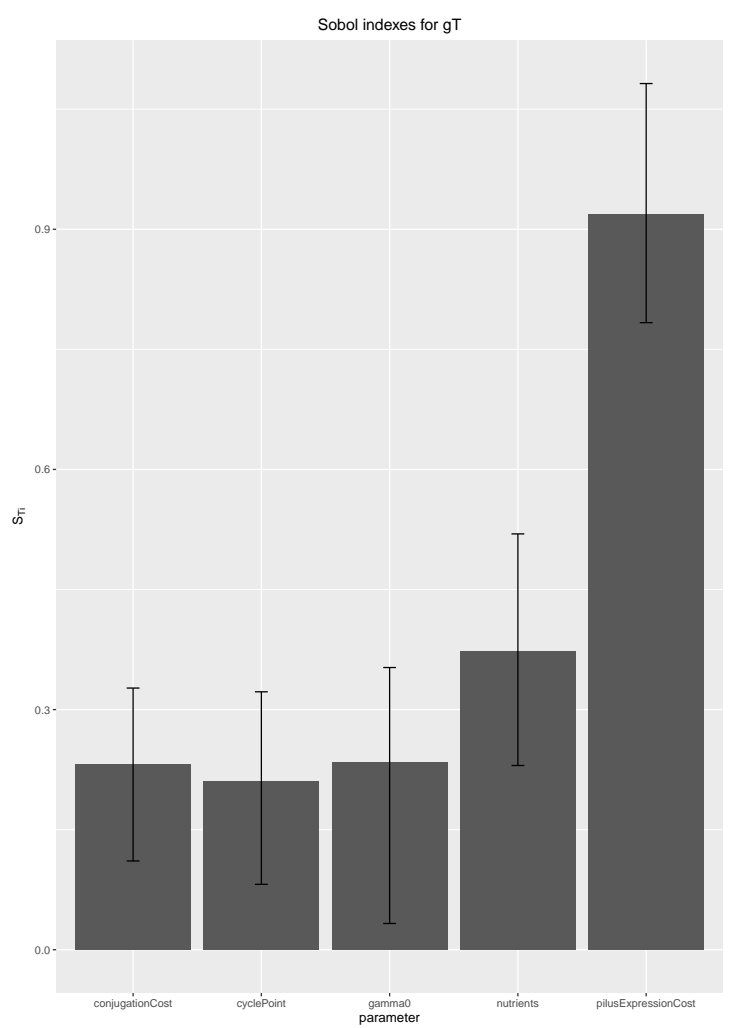

(b) Total order

Figure 8.26: Sensitivity analysis for the plasmid pSU2007 using an initial donor density of 50\%. The first and total order Sobol sensitivity metrics, respectively the subfigures (a) and (b), for the BactoSIM model parameters with respect to the generation time of transconjugant cells (T) output.

Interestingly, the sensitivity indices for the transconjugant cells shown in Figure 8.34 are telling us that most important input factor for the variance in this output variable, differently from previous cases, is the nutrient availability having the remaining parameters a similar importance. That is the correct and expected behavior because the transconjugant cells in this simulations are neither able to express the conjugative machinery nor to accomplish secondary conjugation rounds. Therefore, the only limiting and conditioning factor controlling the doubling time variance of transconjugant cells is the substrate availability.

The overall importance given by the composite output variable shown in Figure 


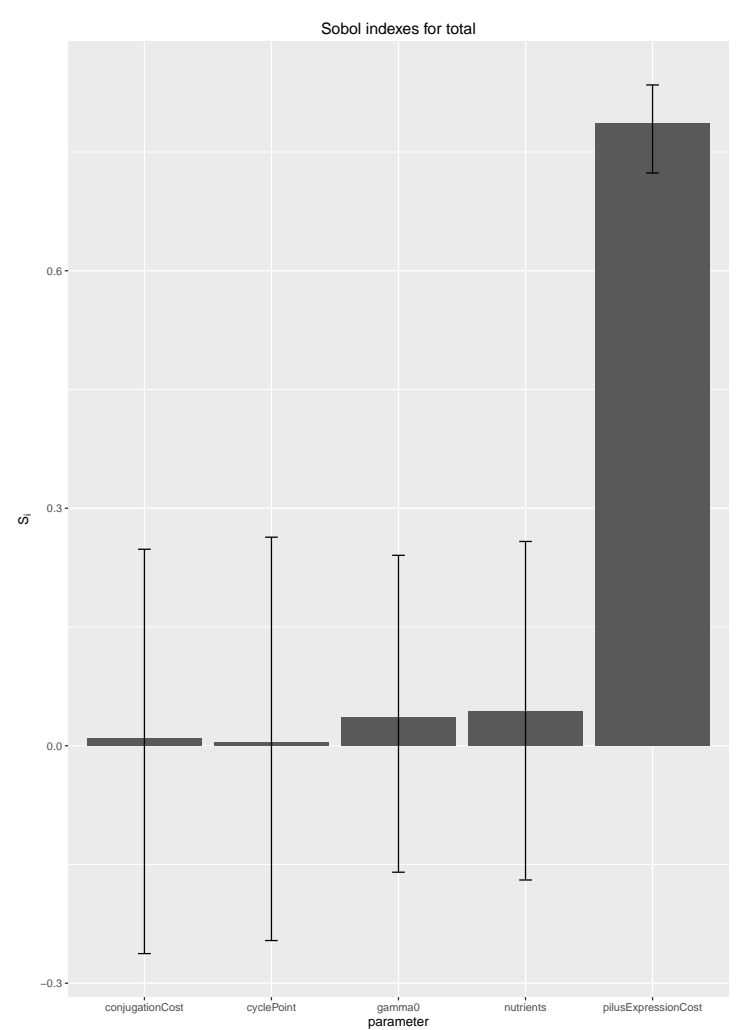

(a) First order

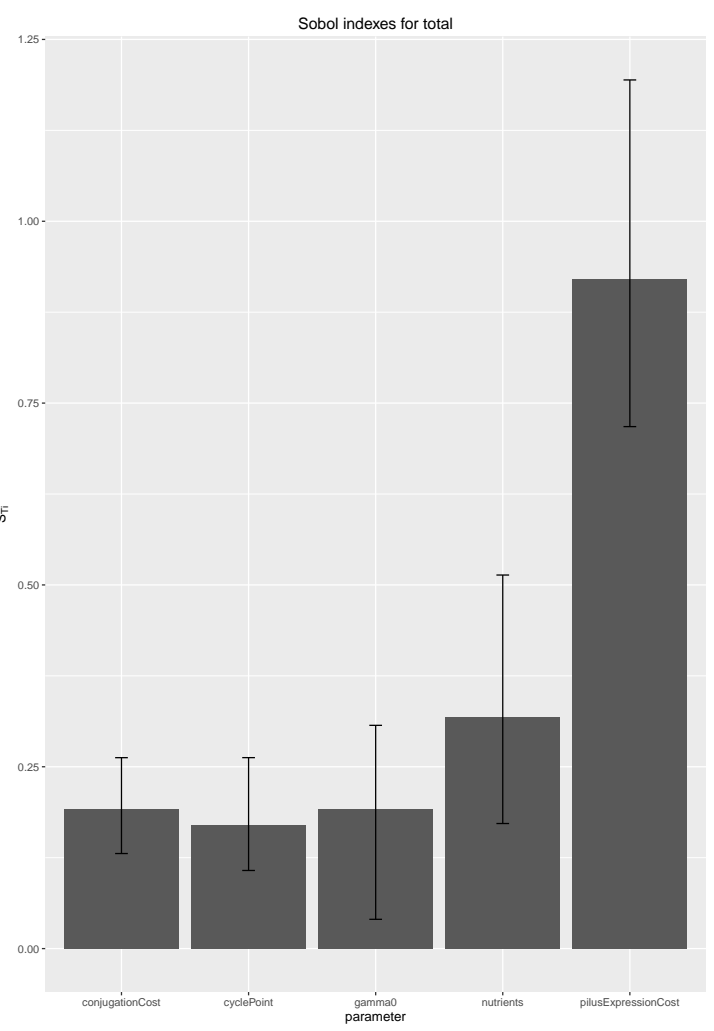

(b) Total order

Figure 8.27: Sensitivity analysis for the plasmid pSU2007 using an initial donor density of $50 \%$. The first and total order Sobol sensitivity metrics, respectively the subfigures (a) and (b), for the BactoSIM model parameters with respect to the composite effect output which is the sum of individual effects over $T /(T+R)$ plus the effects on the generation time of donors and transconjugant cells.

8.35, faithfully accounts that the parameters controlling the three model outputs are in order of importance (total order), the cost of expressing the T4SS apparatus, the nutrient availability, the cost of conjugative transfer, the cycle point and finally $\gamma_{0}$ 


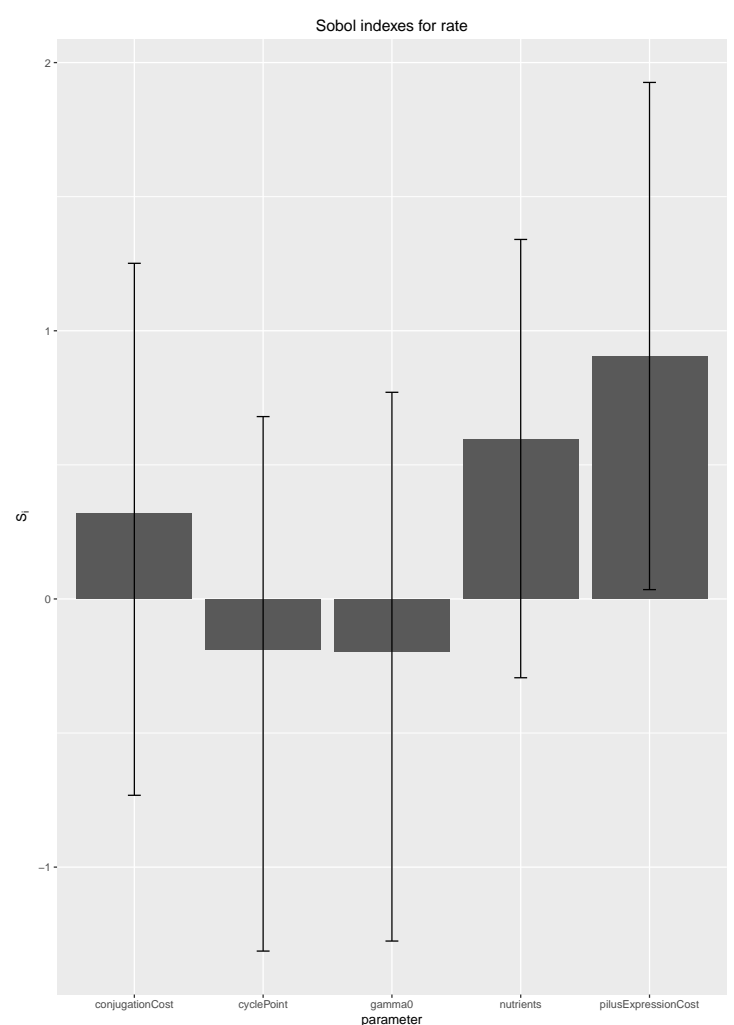

(a) First order

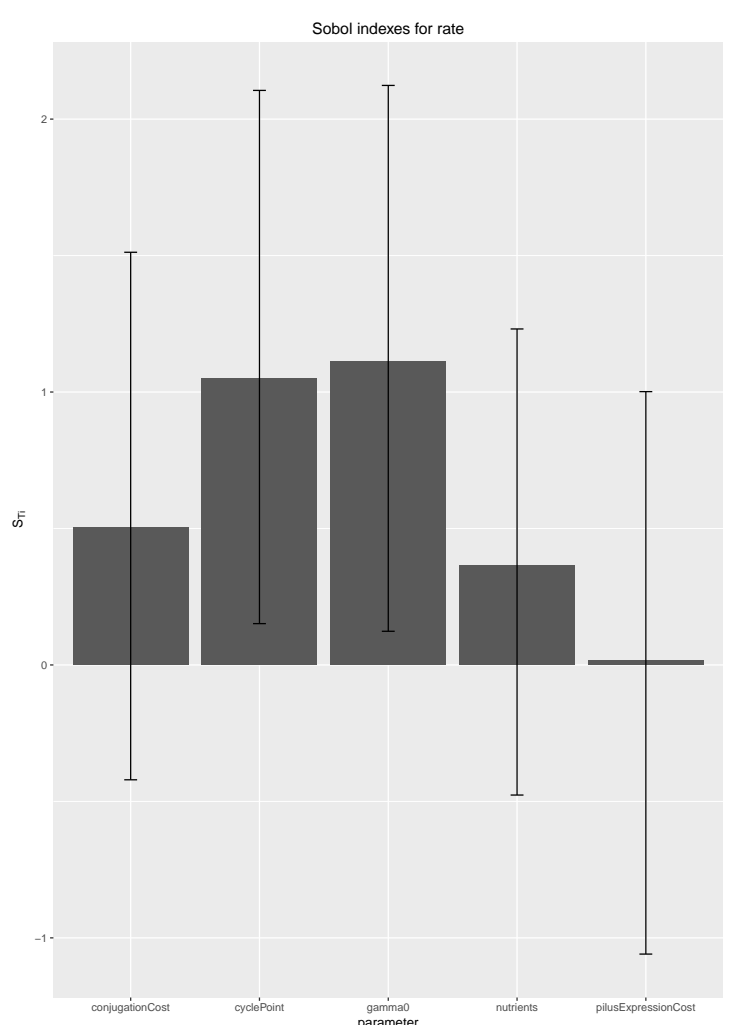

(b) Total order

Figure 8.28: Sensitivity analysis for the plasmid pR388(OriT) using an initial donor density of $5 \%$. The first and total order Sobol sensitivity metrics, respectively the subfigures (a) and (b), for the BactoSIM model parameters with respect to the conjugation rate $T /(T+R)$ output. 


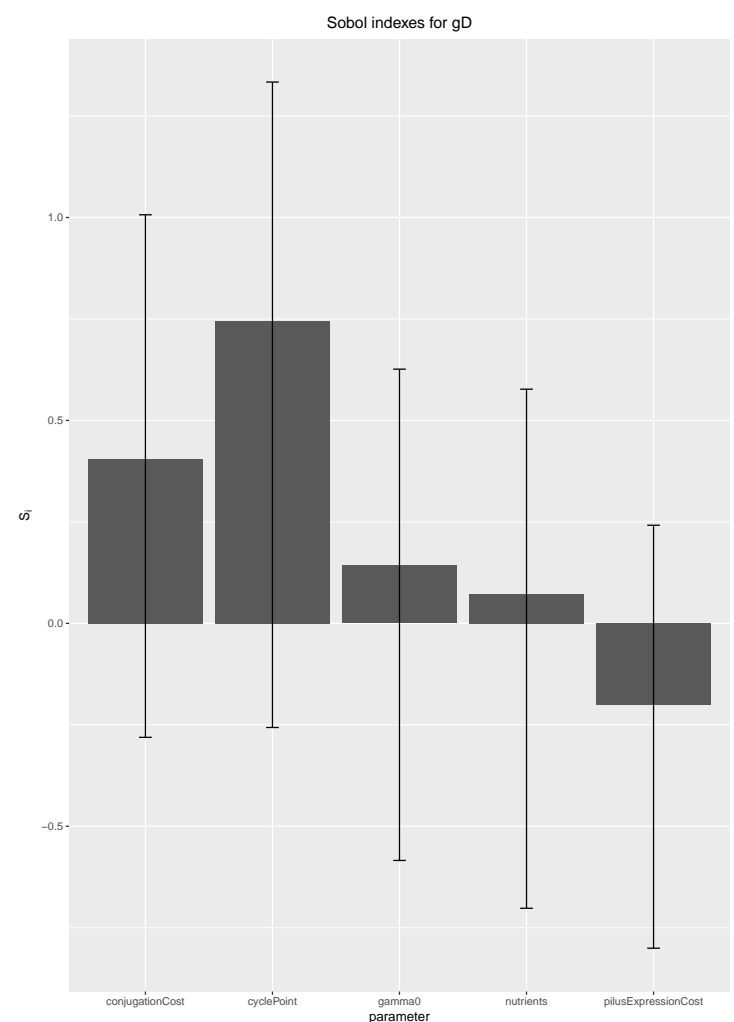

(a) First order

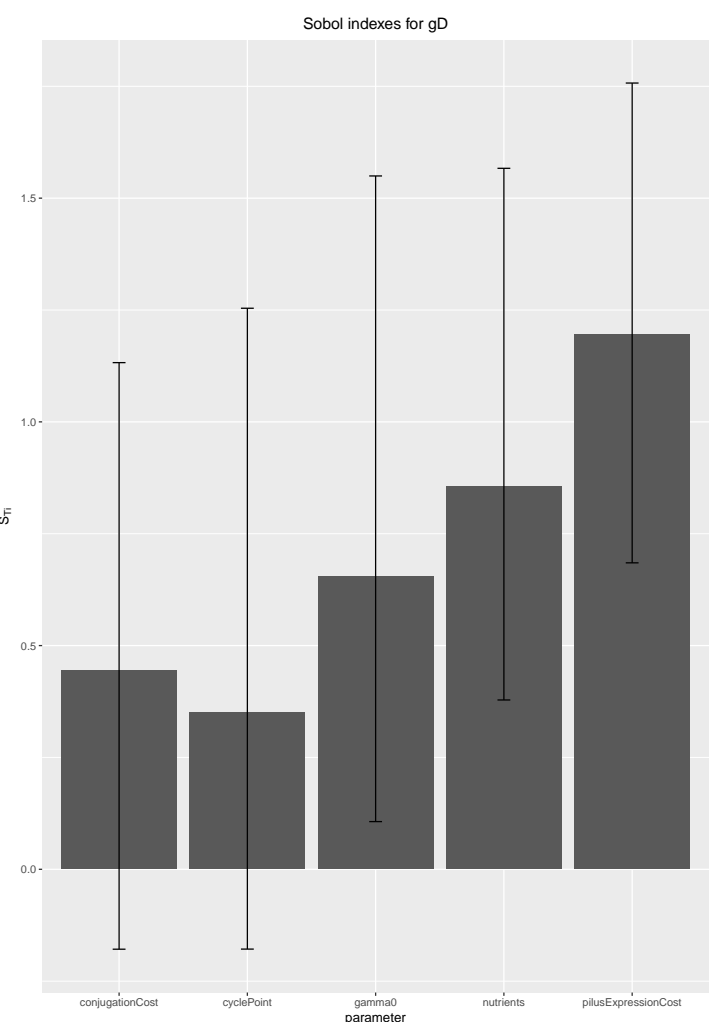

(b) Total order

Figure 8.29: Sensitivity analysis for the plasmid pR388(OriT) using an initial donor density of $5 \%$. The first and total order Sobol sensitivity metrics, respectively the subfigures (a) and (b), for the BactoSIM model parameters with respect to the generation time of plasmid donor cells (D) output. 


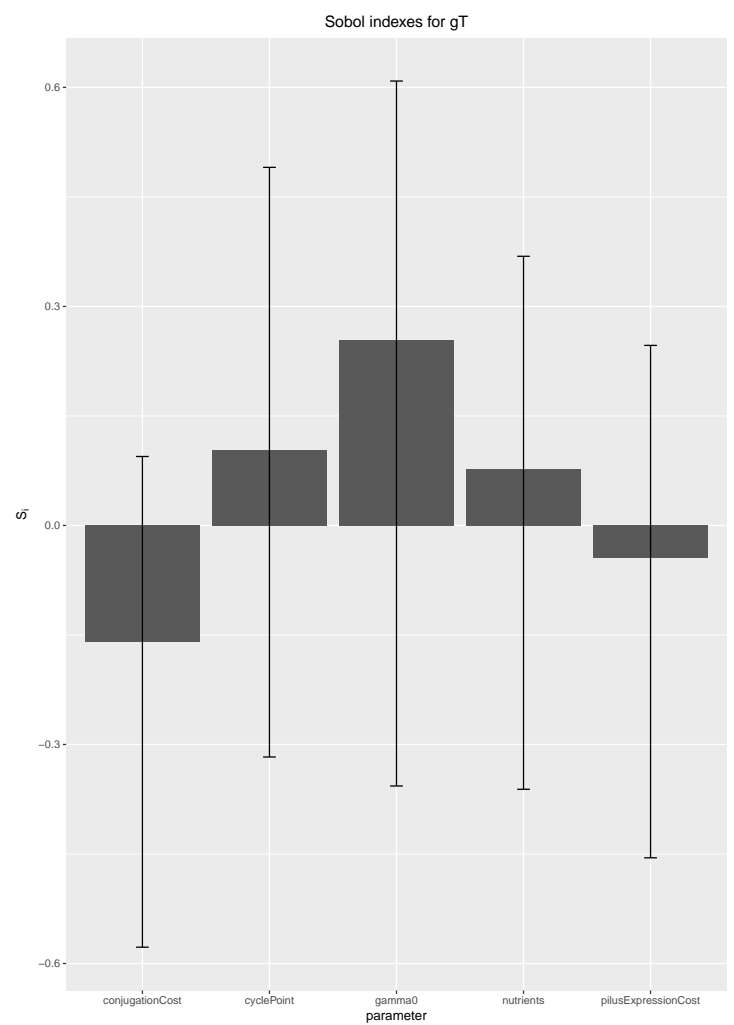

(a) First order

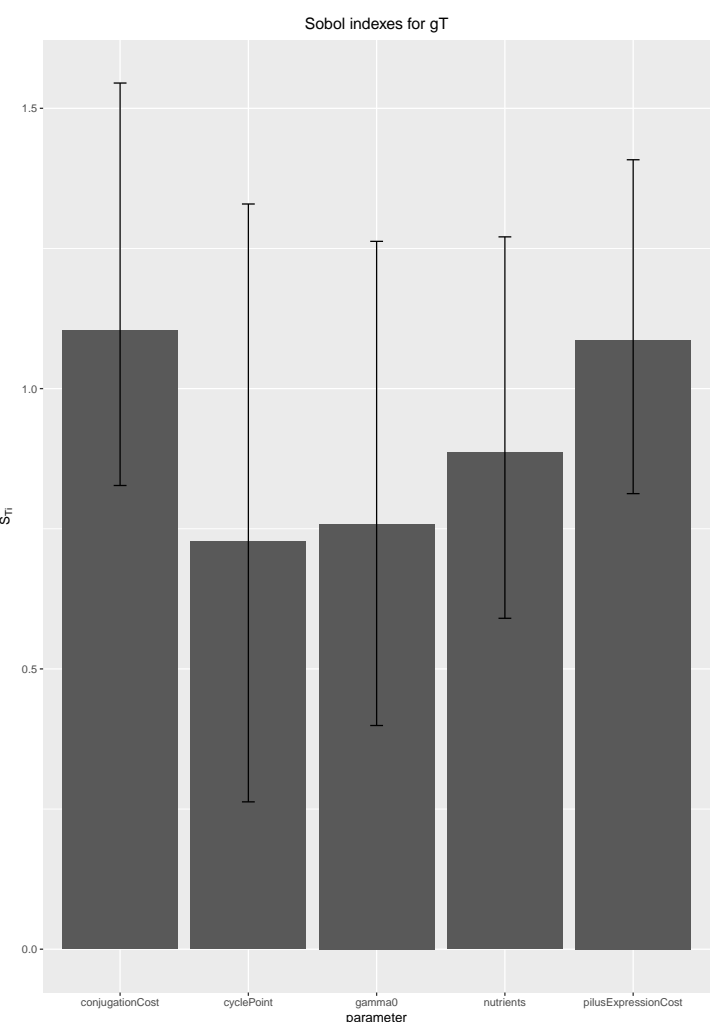

(b) Total order

Figure 8.30: Sensitivity analysis for the plasmid pR388(OriT) using an initial donor density of $5 \%$. The first and total order Sobol sensitivity metrics, respectively the subfigures (a) and (b), for the BactoSIM model parameters with respect to the generation time of transconjugant cells (T) output. 


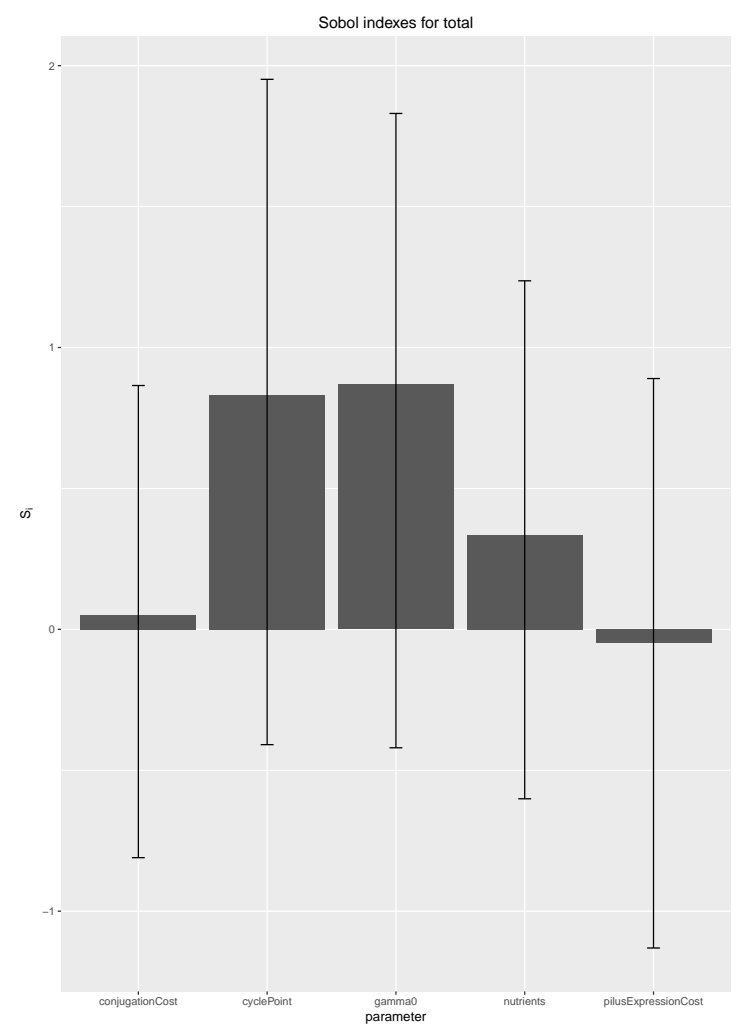

(a) First order

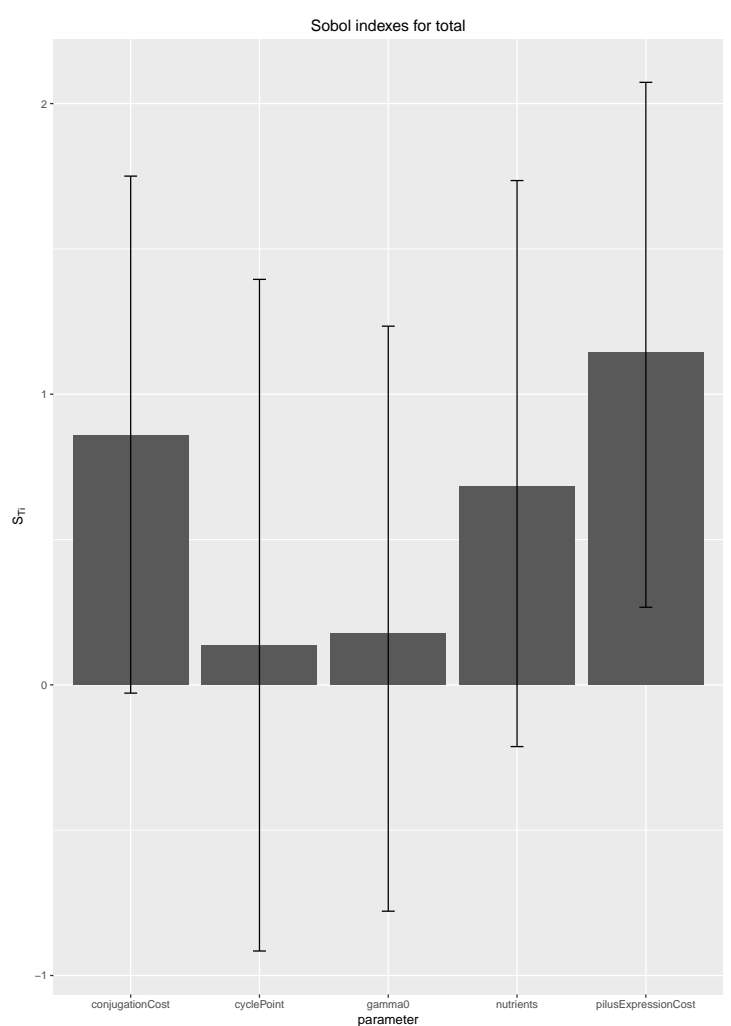

(b) Total order

Figure 8.31: Sensitivity analysis for the plasmid pR388(OriT) using an initial donor density of 5\%. The first and total order Sobol sensitivity metrics, respectively the subfigures (a) and (b), for the BactoSIM model parameters with respect to the composite effect output which is the sum of individual effects over $T /(T+R)$ plus the effects on the generation time of donors and transconjugant cells. 


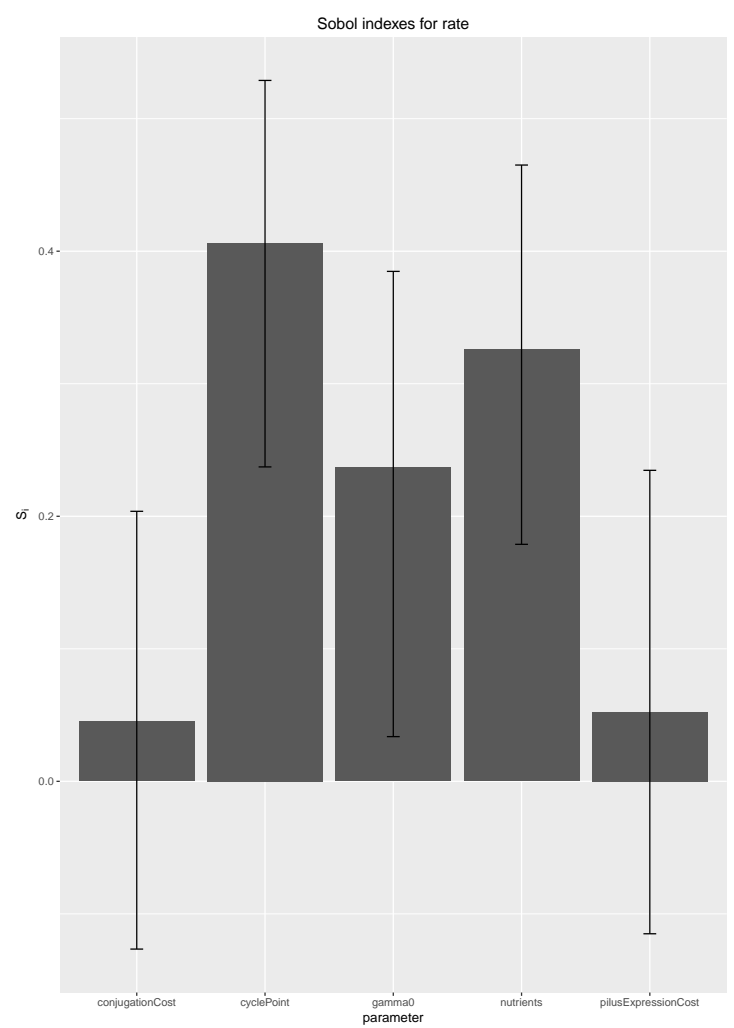

(a) First order

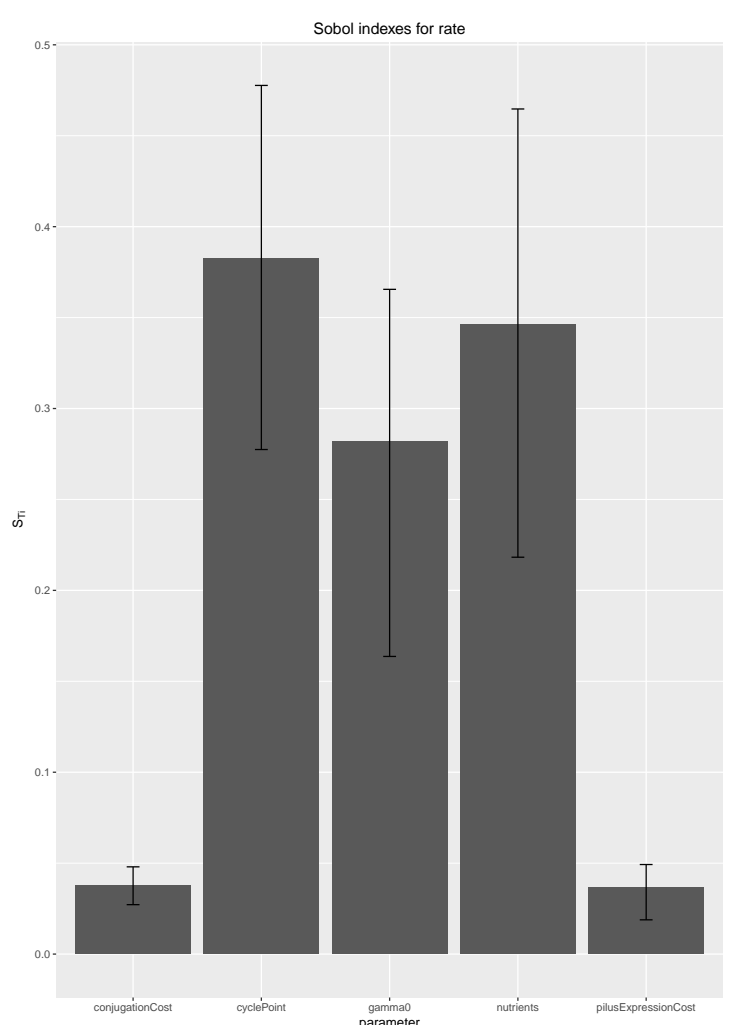

(b) Total order

Figure 8.32: Sensitivity analysis for the plasmid pR388(OriT) using an initial donor density of $50 \%$. The first and total order Sobol sensitivity metrics, respectively the subfigures (a) and (b), for the BactoSIM model parameters with respect to the conjugation rate $T /(T+R)$ output. 


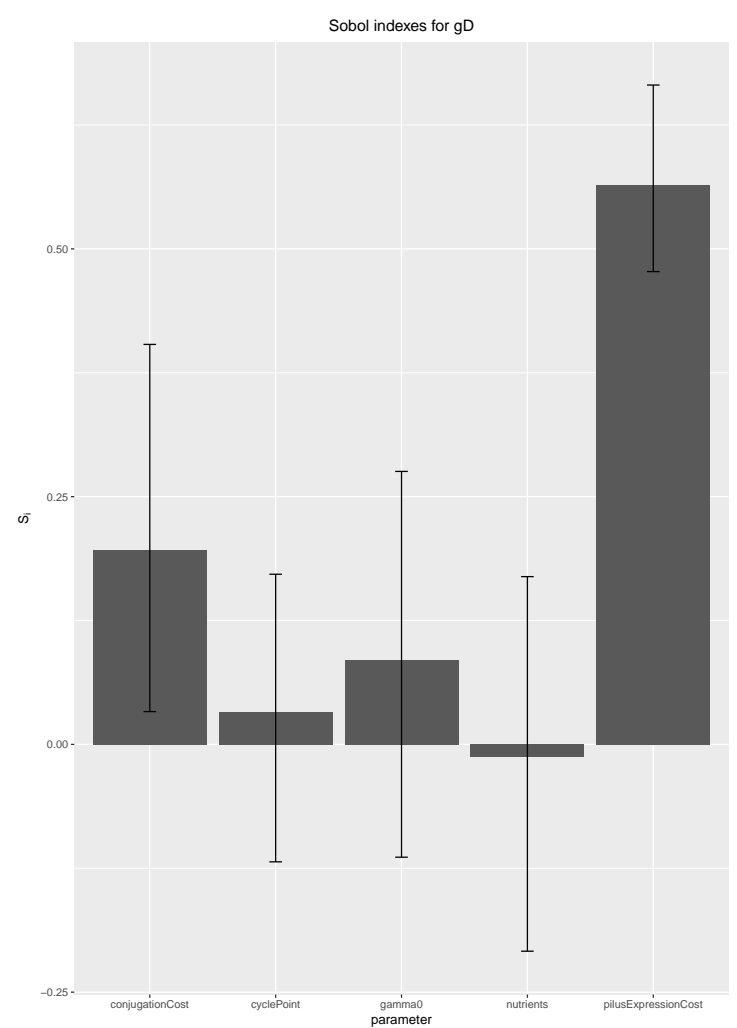

(a) First order

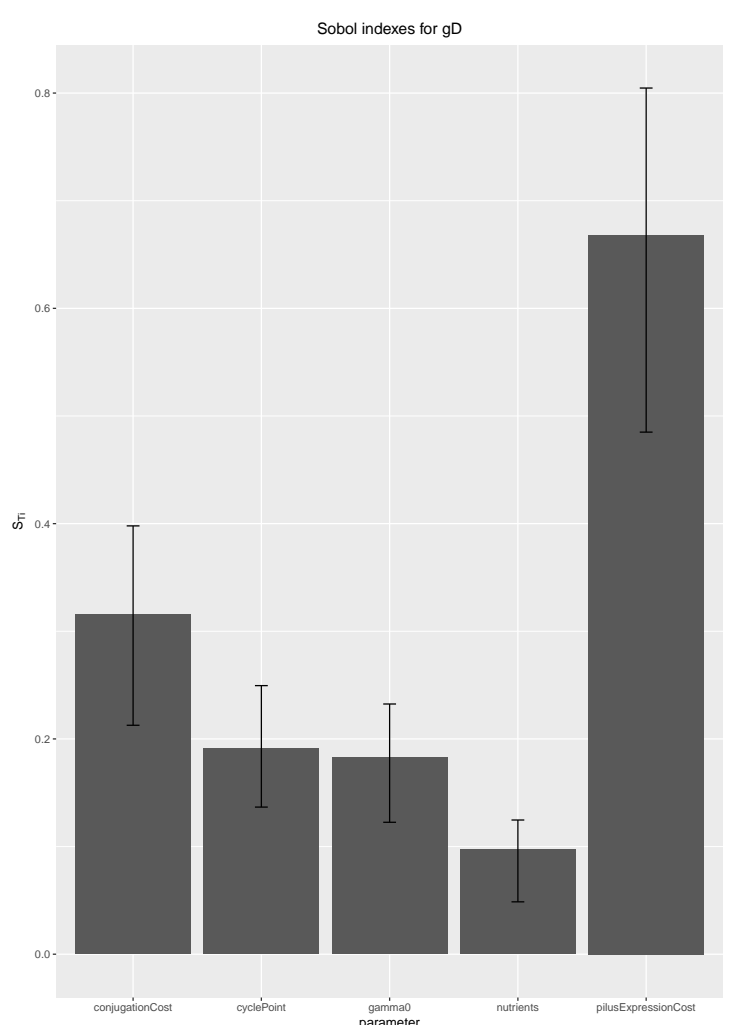

(b) Total order

Figure 8.33: Sensitivity analysis for the plasmid pR388(OriT) using an initial donor density of $50 \%$. The first and total order Sobol sensitivity metrics, respectively the subfigures (a) and (b), for the BactoSIM model parameters with respect to the generation time of plasmid donor cells (D) output. 


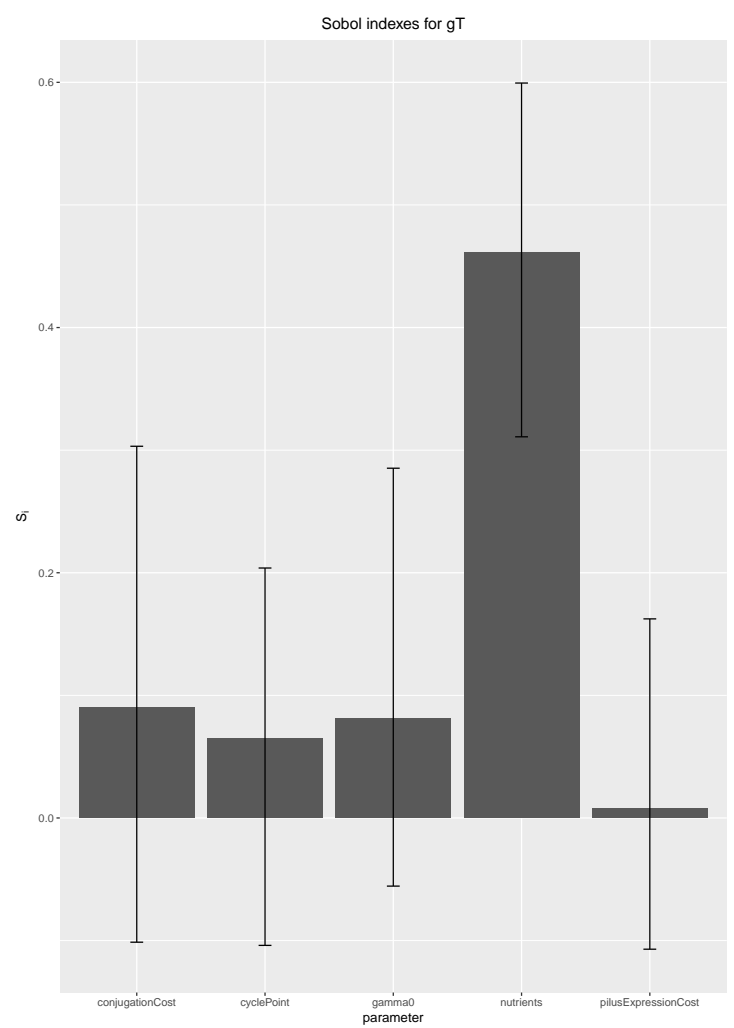

(a) First order

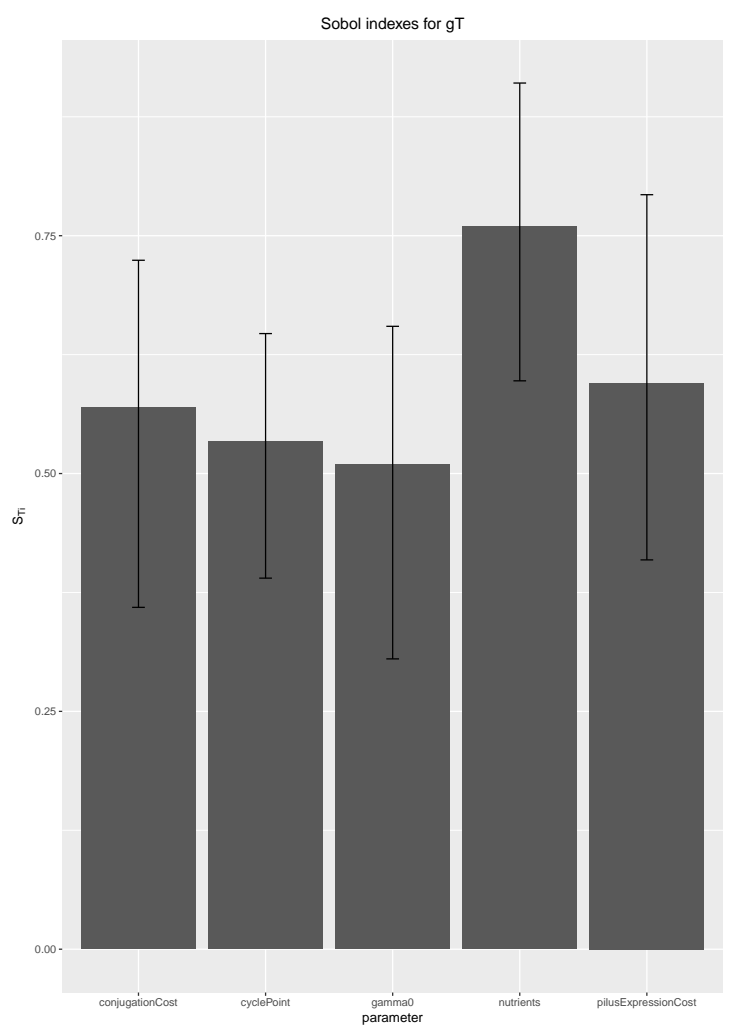

(b) Total order

Figure 8.34: Sensitivity analysis for the plasmid pR388(OriT) using an initial donor density of $50 \%$. The first and total order Sobol sensitivity metrics, respectively the subfigures (a) and (b), for the BactoSIM model parameters with respect to the generation time of transconjugant cells (T) output. 


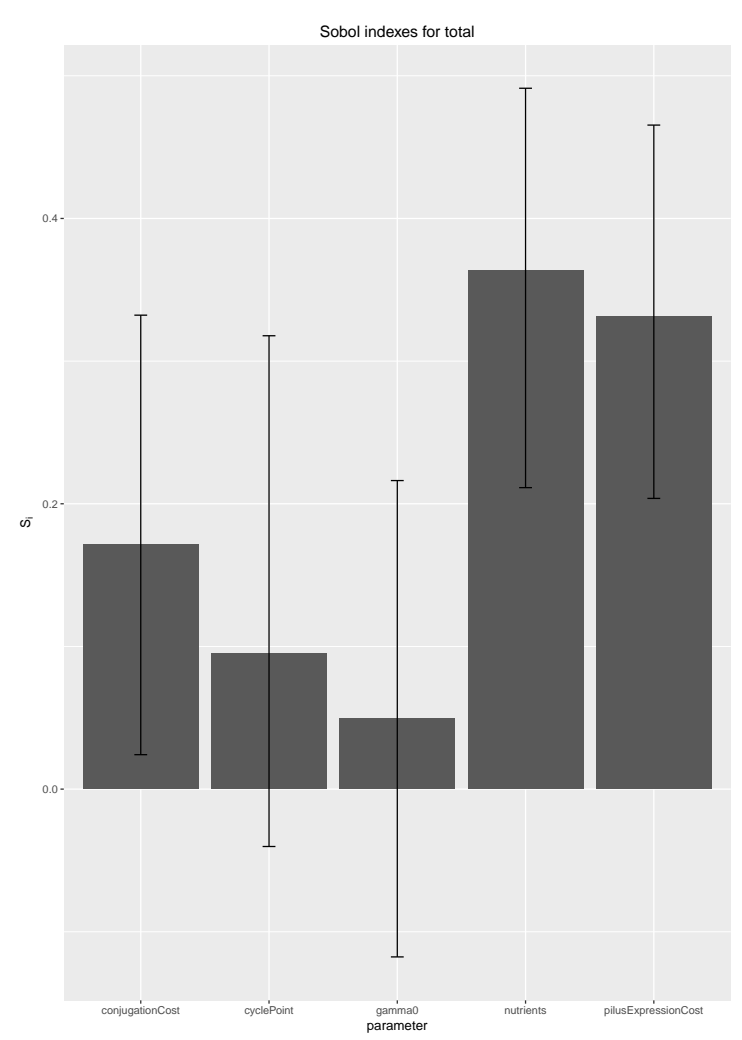

(a) First order

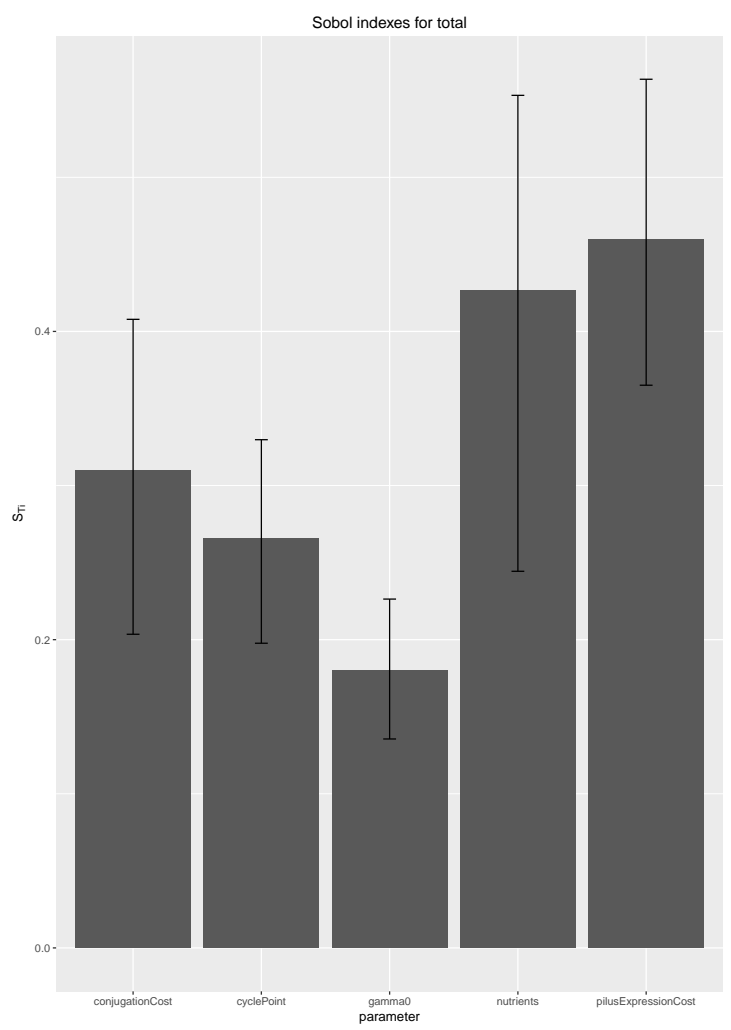

(b) Total order

Figure 8.35: Sensitivity analysis for the plasmid pR388(OriT) using an initial donor density of $50 \%$. The first and total order Sobol sensitivity metrics, respectively the subfigures (a) and (b), for the BactoSIM model parameters with respect to the composite effect output which is the sum of individual effects over $T /(T+R)$ plus the effects on the generation time of donors and transconjugant cells. 


\subsection{Summary}

In this chapter we have analyzed systematically the BactoSIM model output using the parameter estimation and the sensitivity analysis tools for trying to understanding the sources of uncertainty enclosed in the model results. We have performed the preliminary analysis using the Morris screening method, which provide good results and is not too much expensive, computationally speaking. The basic objective for applying this technique is to check if the model parameter effectively contributes for the output variability and we have found that it was one of the most important parameters of BactoSIM.

Afterwards, the next step was to order the three model types according to its contributions for the fitness cost, that is to say, the distance between the simulated and the observed data. We have used an optimization metaheuristic for undertake this step and we have found that the model type 3 provided the best fit for the experimental data. We have applied the ANOVA test for verifying whether the fitness outputs for the three models were statistically distinct. Our results points that the better way to model bacterial conjugation is making the period where a donor is able to conjugate located at a small window late in the bacterial cell cycle. On the average, the window where most conjugative events will occur is located at least $50 \%$ of bacterial generation time with a small coefficient of variation not greater than a $10 \%$ of the average value. With these values the best fit to the experimental data is achieved.

Despite of the fact that the experimental values of conjugation rates $T /(T+R)$ for the whole population can present a broad range of variation depending on the plasmid type or if they are repressed or depressed, the local values are consistently low. By local values we mean the number of conjugative events during the interval between two successive divisions, that is to say the intrinsic conjugation rate $\left(\gamma_{0}\right)$. In other words, the values of $\gamma_{0}$ required for achieving a good fit to the experimental data are on average, equal to one event per cell cycle with an upper bound lower than two events (Tables 8.5). This seems to be consequence of spatial structure leading to physical separation of donors to their potential susceptible recipients but this is compensated by the vertical transfer allowing the plasmids to become established, even though the 
horizontal infection spread becomes slightly limited.

Additionally, the most significant contribution to the global dynamics of the model is the total delay required to forward the plasmid, at least for derepressed and mobilizable plasmids. That is to say, the parameter $\gamma_{1}$ (cellCycle), according to the rank of Morris screening method (Tables 8.2, 8.3 and 8.4) is the most significant in two cases. In the case of repressed plasmids, such as the pAR118 used in this study, $\gamma_{1}$ is the fifth in importance. Furthermore, the results obtained from the Sobol method (Figures 8.20 , 8.24 and 8.32) has confirmed these results for the derepressed plasmid pSU2007 (initial donor densities of $5 \%$ and $50 \%$ ) and for the mobilizable plasmids R388(OriT) (initial donor density of $50 \%$ ).

The model output for three different plasmids has been evaluated using the Sobol method, at two initial donor concentrations in order to emulate the experimental setup[dCRC $\left.{ }^{+} 12\right]$. The global sensitivity analysis results in some of the situations evaluated here were extremely clear, as in the case of de-repressed plasmid pSU2007 which show the importance of the cellCycle in the model output variability. The composite metric shows a somehow counterintuitive result about the effect of the pilusExpressionCost parameter on the generation time of both donor and transconjugant cells. That possible behavior has two explanations. The first explanation is related to the model structure which initializes the initial donor cells population with some random ratio of them having an already stable level of T4SS expression, but part of the initial population has to invert some metabolic effort to reach the stable level of expression. The second explanation has to do with the competition between donors and transconjugants as the transconjugant cells need to expend much more energy for expressing the conjugative apparatus, allowing the donor outcompete them for space and nutrients which also explain the importance of nutrient output variables.

Generally speaking, the sensitivity indices for the conjugation rate output are clearly pointing out that cyclePoint $\left(\gamma_{1}\right)$ parameter has a very significant impact on the output variance. In some cases, as can be observed for the de-repressed plasmid it

\footnotetext{
${ }^{8}$ The nutrient dependency is an structural part of the model and therefore it is an expected behavior. On the other hand, the cycle dependency is an emergent property.
} 
was the second most important behind the nutrient input factor which could be attributed to a strong competition between donors and transconjugants, as well as, to the vertical transmission. The mobilizable plasmids, which are basically performing one conjugation round, shows a canonical behavior being the cycle parameter the most important. The repressed plasmid are somewhat more noise and certainly the experiments may require more replications, but also seems to indicate $\gamma_{1}$ as significant for the conjugation rate $T /(T+R)$ output. 


\section{Part V}

Conclusions and Future Research 

Chapter Conclusions 
In this thesis, we have presented a study based on the computational simulation for the bacterial conjugation kinetics in structured environments. This study uses an individual-based modeling approach for capturing some of the fundamental structural properties governing the global dynamics of the plasmid spread patterns. The model relies on the conceptualization of the cell-cycle dependency as well as the associated metabolic costs and delays related to the conjugation process. In other words, the model express the idea that horizontal gene transfer is somehow linked to the host cell cycle requiring some amount of time, relative to the interval between successive birth and divisions, for the occurrence of conjugative events. In order to carry out a conjugative transfer, bacterial cells devote some amount of their energetic resources which is collectively denoted as the plasmid metabolic burden encompassing the metabolic costs and delays increasing the cell cycle length of plasmid donors. This dependency constitutes a feedback loop, as the conjugation depends on the cell cycle which in turn, depends on the fitness penalization derived from conjugation activity.

The former idea is conceptually simple and it could even be assumed axiomatically as being structurally consistent with the biological reality of the underlying system. The complete process of validating this mechanistic model about how and when the conjugation occurs, was carried out in two steps. The first step was a simple exploratory analysis, consisting in the visual assessment comparing the model outputs with the experimental time series for the conjugation rates $T /(T+R)$. The comparison was carried out using different approaches to express the conjugation rule. The rationale behind the methodology was to identify and build up three falsifiable hypotheses which were non-overlapping representations of the conjugation process. The first hypothesis, considered the conjugation as being completely independent of cell cycle in such extent that the conjugative event occurred immediately when a suitable recipient is found by any proficient donor. The second approach consisted in the assumption that conjugative events were evenly distributed across the cell cycle and, was implemented using a uniform distribution. Finally, the third one dictates that the conjugation is linked to the cycle occurring on a restrict temporal window and implemented using a random number with normal distribution. These three rules share all the common model sub-process for the vegetative cellular behavior and the colony expansion. The initial results obtained with the simple experimental setup have shown that the last one provides the most natural adjustment to the experimental data. 
Subsequently, recognizing the need of a more formal approach for validating the simulation outputs, the model was submitted to a more throughout assessment using the parameter estimation and the sensitivity analysis methodologies introduced previously in this work. The first methodology consisted in expressing the discrepancies between the observed and the simulated values as an optimization problem which is amenable for the application of metaheuristic-based minimization algorithms.

By recognizing the difficulties of finding an acceptable optimum in a reasonable amount of time, owing to the computational cost of simulating the minimal number of agents for obtaining representative results, we have tried to use heuristic search algorithms that requires few evaluations. These algorithms have been adapted for providing an additional output with the collection of visited points in the search space for producing a map with the solutions with the best fitness values. These maps promote the identifications of the real solution zones being useful for an exploratory analysis than a single point estimate with the local optima found for the problem. The second technique, the global sensitivity analysis serves for assessing quantitatively the effects of the model inputs on the output variance constituting a fundamental piece for the what-if analysis allowing a broader model comprehension.

The both methodologies used together can greatly improve the level of confidence of the conclusions and insights extracted from the model results although it is possibly a terrible error-prone and frustrating task to extract trustworthy conclusions exclusively from computational simulation outputs. It is especially true when the reference experimental data available and used for the parameter estimation come from whole population studies and the values being analyzed make reference to intracellular properties which are complicated or impossible to elucidate experimentally. All the software developed in the context of this thesis for simulating the conjugation dynamics (BactoSIM) and the R packages EvoPER and R/Repast are opensource and released under the MIT license.

We recognize the fact and assume that the conclusions should be taken with absolute care but nevertheless empathize the importance of the results and the qualitative explanations extracted from our computational model. The model building is viable because of the $80 / 20$ Pareto principle holds for most of the physical and biological systems with a limited number of system parameters explaining the most part of system trajectories. In the end, there is no need that model to be true, it is enough just to 
provide a marginal improvement about the structural parts of a system.

In the specific framework of this work, we estimate that a significant advance has been made in the local to global and in the systemic knowledge of conjugation process to the extent that some of main effects have been ranked quantitively using a sound computational and analytical methodology. That approach could be considered at least as a weak evidence in the context of biological systems, allowing us to be assertive in the importance of considering the linking between cell cycle, the intrinsic conjugation rates and metabolic costs to the global dynamics observed. These results are referring to the surface attached bacterial colonies but certainly can also be extrapolated for liquid cultures.

Admittedlly, we cannot state with a complete confidence the true values of these parameters but we could be satisfied with being confident about their relative magnitudes and that at least that the simulated values are in the same order of magnitude and that the estimated parameters are close to their real values. Again, the size and scale are important physical constraints and differently from other host-parasite or host-symbiont associations which are separated by orders of magnitude which makes the cycle dependence and the relative impact on the metabolic cost negligible, the case of bacterial plasmids the both magnitudes are very close and the effects on the epidemic dynamics is a factor to be taken into account. 
Chapter 10 Future work 
In this work, we have outlined what we consider, based on the analysis of the model outputs, the most basic tenets of what conforms the kinetics of plasmid spread, namely cycle dependency and the simple metabolic cost model affecting the cell cycle. The validation of these two components-model have shown an acceptable agreement with the experimental data, both quantitatively and qualitatively. The observed global pattern developed during the growth of bacterial colony seems to indicate that we have achieved a structurally sound model. Of course, it is needless to say that, as in the case of any research work, results are far from being somehow definitive. Additionally, it is necessary to improve and extend the initial aspects presented here, relating the plasmid structural characteristics and its impact on the observed conjugation kinetics.

With respect to the experimental validation it would be very important, in order to avoid the confirmation bias, to implement an experimental design for corroborate that the hypothesis about the linking between conjugation and cell cycle actually holds. Therefore, it will require some laboratory work from microbiologists for designing several conjugation essays for the same combination of plasmid and bacterial strain using the culturing medium for tuning the growth at different speeds for corroborate or refute the null hypothesis which fundamentally states that, in average, conjugation rates are similar, independently of the bacterial doubling time and consequently linked to the cell cycle.

The second mentioned item, is another clear path for continuing and improving this work. Fundamentally, the current version of the model serves as the base for elucidating some intracellular aspects of plasmid-host interaction but it is no operational for predicting what kind of behavior should be expected without first executing a complete experimental conjugation essay. Naturally, it is not practical and an ideal model should be able to provide at least some basic predictions using the data taken from the plasmid structural aspects such as the amount of DNA, the annotated gene products in such extent that it can be used for computing an energy budget and its interference on the overall cellular energetic profile. This would be a really useful improvement making the model suitable for prediction the conjugation dynamics even on unculturable bacterial strains or in uncontrollable natural environments.

Finally, but not least important some effort is still required on the engineering aspects of the model software. The current versions encompass a disperse collection of many model implementations with slight variations for verifying some specific aspect 
of the conjugation process. Thus, it is necessary to consolidate these versions based on the results as well as improving some of the model sub-process. Additionally, it would be also a significant improvement, being able to accelerate the model execution speed in order to simulate larger populations and making a better physical dynamics for the aspects related to the bacterial growth sub-process dynamics. 


\section{Bibliography}

[AF13] Denis Arutyunov and Laura S. Frost, F conjugation: Back to the beginning, Plasmid 70 (2013), no. 1, 18-32.

[AFNKB09] Maksat Ashyraliyev, Yves Fomekong-Nanfack, Jaap A. Kaandorp, and Joke G. Blom, Systems biology: Parameter estimation for biochemical models, 2009, pp. 886-902.

[AH93] T.H. Andres and W.C. Hajas, Using iterated fractional factorial design to screen parameters in sensitivity analysis of a probabilistic risk assessment model, 1993.

[AIC08] Keith L. Aubin, Bojan Ilic, and Harold G. Craighead, Micro- and Nanoelectromechanical Sensors, Handbook of Biosensors and Biochips, John Wiley \& Sons, Ltd, Chichester, UK, mar 2008.

[Álv12] Jean Pierre Arteaga Álvarez, Enhancing idynomics framework to simulate rod-shape bacterial colonies growth, Master's thesis, Facultad de informática(UPM), 2012.

[ASD06] Sonja-Verena Albers, Zalán Szabó, and Arnold J. M. Driessen, Protein secretion in the Archaea: multiple paths towards a unique cell surface, Nature Reviews Microbiology 4 (2006), no. 7, 537-547.

[BA77] James Vere Beck and Kenneth J. Arnold, Parameter estimation in engineering and science, Wiley series in probability and mathematical statistics, Wiley, New York, 1977. 
[BBM10] Zeynep Baharoglu, David Bikard, and Didier Mazel, Conjugative DNA transfer induces the bacterial SOS response and promotes antibiotic resistance development through integron activation., PLoS genetics 6 (2010), no. 10, e1001165+.

[BC93] Stavros. Busenberg and Kenneth. Cooke, Vertically Transmitted Diseases : Models and Dynamics, Springer Berlin Heidelberg, 1993.

[BCNN09] Jerry Banks, John S. Carson, Barry L. Nelson, and David M. Nicol, Discrete-Event System Simulation (5th Edition), 5 ed., Prentice Hall, July 2009.

[BD87] George Box and Norman Draper, Empirical model-building and response surfaces. Wiley series in probability and mathematical statistics., 1 ed., Wiley, New York, 1987.

[Ber02] Luděk Berec, Techniques of spatially explicit individual-based models: construction, simulation, and mean-field analysis, Ecological Modelling 150 (2002), no. 1-2, 55-81.

[BFW ${ }^{+}$09] G. J. Bean, S. T. Flickinger, W. M. Westler, M. E. McCully, D. Sept, D. B. Weibel, and K. J. Amann, A22 Disrupts the Bacterial Actin Cytoskeleton by Directly Binding and Inducing a Low-Affinity State in MreB, Biochemistry 48 (2009), no. 22, 4852-4857.

[BJST ${ }^{+}$94] Eshel Ben-Jacob, Ofer Schochet, Adam Tenenbaum, Inon Cohen, Andras Czirók, and Tamas Vicsek, Generic modelling of cooperative growth patterns in bacterial colonies, Nature 368 (1994), no. 6466, $46-49$.

[BK96] Bert Bettonvil and Jack P C Kleijnen, Searching for important factors in simulation models with many factors: Sequential bifurcation, EUROPEAN JOURNAL OF OPERATIONAL RESEARCH ELSEVIER European Journal of Operational Research 96 (1996), 180-194.

[Bla58] R. E. Blackith, Nearest-Neighbour Distance Measurements for the Estimation of Animal Populations, Ecology 39 (1958), no. 1, 147. 
[BLL00] Carl T. Bergstrom, Marc Lipsitch, and Bruce R. Levin, Natural Selection, Infectious Transfer and the Existence Conditions for Bacterial Plasmids, Genetics 155 (2000), no. 4, 1505-1519.

[BLS13] Ilhem Boussaïd, Julien Lepagnot, and Patrick Siarry, A survey on optimization metaheuristics, Information Sciences 237 (2013), 82-117.

[Boc03] Nino Boccara, Modeling Complex Systems (Graduate Texts in Contemporary Physics), 1 ed., Springer, November 2003.

[Bon02] E. Bonabeau, Agent-based modeling: Methods and techniques for simulating human systems, Proceedings of the National Academy of Sciences 99 (2002), no. Supplement 3, 7280-7287.

[BPB ${ }^{+}$97] F R Blattner, G Plunkett, C A Bloch, N T Perna, V Burland, M Riley, J Collado-Vides, J D Glasner, C K Rode, G F Mayhew, J Gregor, N W Davis, H A Kirkpatrick, M A Goeden, D J Rose, B Mau, and Y Shao, The complete genome sequence of Escherichia coli K-12., Science (New York, N.Y.) 277 (1997), no. 5331, 1453-62.

[BSK ${ }^{+}$13] David A. Baltrus, M. Syvanen, P.J. Keeling, J.D. Palmer, G. Schonknecht, et Al., N.A. Moran, T. Jarvik, S. Yoshida, et Al., T.J. Treangen, E.P.C. Rocha, S. Schaack, et Al., C. Park, J. Zhang, H.H. Chou, et Al., H. Engelberg-Kulka, G. Glaser, J.C. Diaz-Ricci, M.E. Hernández, G.A. Gonçalves, et Al., E. Rensburg, et Al., H. Heuer, et Al., M. Subbiah, et Al., E. Harrison, M.A. Brockhurst, M. Gaillard, et Al., T. Sato, H. Kuramitsu, C. Tagwerker, et Al., M. Itaya, et Al., R. Sorek, et Al., C.J. Creevey, et Al., O. Cohen, et Al., C. Pál, et Al., B. Green, et Al., L. Rabinovich, et Al., F. Hayes, A.E. Darling, et Al., T.D. Matthews, et Al., K.A. Geiler-Samerotte, et Al., B. Jürgen, et Al., H.P. Narra, et Al., D.A. Drummond, C.O. Wilke, M.A. Kohanski, et Al., J.G. Bragg, A. Wagner, I. Shachrai, et Al., A. Wellner, U. Gophna, C. Pal, et Al., T. Vavouri, et Al., J.R. Yang, et Al., J.F. Gout, et Al., J. Bonomo, R.T. Gill, Y. Chen, et Al., M. Shintani, et Al., I.J. Schalk, et Al., R.K. Azad, J.G. Lawrence, H. Akashi, T. Gojobori, R.C. Baños, 
et Al., R. Raghavan, et Al., H. Gingold, Y. Pilpel, D. Agashe, et Al., T. Tuller, et Al., O.X. Cordero, P. Hogeweg, B. Humphrey, et Al., A. Singer, et Al., R.F. Silva, et Al., M. Miyakoshi, et Al., F.R. Slater, et Al., V. Enne, et Al., T.G. Platt, et Al., J. Grote, et Al., J.J. Grzymski, A.M. Dussaq, R.J. Woods, et Al., O. Tenaillon, et Al., J.G. Lawrence, H. Ochman, M.J. Lercher, C. Pal, D. Amoros-Moya, et Al., M-C. Lee, C.J. Marx, H-H. Chou, C.J. Marx, M. Sota, et Al., C. Dahlberg, L. Chao, P.E. Turner, et Al., L. Dethlefsen, T.M. Schmidt, M-C. Lee, C.J. Marx, D.A. Drummond, C.O. Wilke, M. Doyle, et Al., C.T. Skennerton, et Al., V. Petrova, et Al., E. Perrody, et Al., S.A. Godfrey, et Al., S.P. Otto, P. Zünd, G. Lebek, G.F. Hatfull, J. Liu, et Al., P.W. Harrison, et Al., C. Smillie, et Al., D.A. Baltrus, and et Al., Exploring the costs of horizontal gene transfer, Trends in Ecology \& Evolution 28 (2013), no. 8, 489-495.

$\left[\mathrm{BSP}^{+}\right.$98] V Burland, Ying Shao, Nicole T. Perna, Guy Plunkett, Frederick R. Blattner, and Heidi J. Sofia, The complete DNA sequence and analysis of the large virulence plasmid of Escherichia coli O157:H7, Nucleic Acids Research 26 (1998), no. 18, 4196-4204.

[BSRP15] David Beneš, Petr Sosík, and Alfonso Rodríguez-Patón, An Autonomous In Vivo Dual Selection Protocol for Boolean Genetic Circuits, Artificial Life 21 (2015), no. 2, 247-260.

[BTH06] Michael Begon, Colin R. Townsend, and John L. Harper, Ecology : from individuals to ecosystems, Blackwell Pub, 2006.

[CCD05] Inês Chen, Peter J. Christie, and David Dubnau, The ins and outs of DNA transfer in bacteria., Science (New York, N.Y.) 310 (2005), no. $5753,1456-1460$.

[CCS01] Stephen Cooper, Cooper, and Stephen, Bacterial Reproduction and Growth, Encyclopedia of Life Sciences, John Wiley \& Sons, Ltd, Chichester, UK, apr 2001. 
[CCS07] Francesca Campolongo, Jessica Cariboni, and Andrea Saltelli, An effective screening design for sensitivity analysis of large models.

[CHGS17] Yangrong Cao, Morgan K. Halane, Walter Gassmann, and Gary Stacey, The Role of Plant Innate Immunity in the Legume-Rhizobium Symbiosis, Annual Review of Plant Biology 68 (2017), no. 1, annurevarplant-042916-041030.

[Cle12] Maurice Clerc, Standard Particle Swarm Optimisation.

[Cra07] Michael J. Crawley, The R Book, 1 ed., Wiley, jun 2007.

[dB16] Piet A J de Boer, Classic Spotlight: Discovery of ftsZ., Journal of bacteriology 198 (2016), no. 8, 1184.

[DBS06] Marco Dorigo, Mauro Birattari, and Thomas Stutzle, Ant colony optimization, IEEE Computational Intelligence Magazine 1 (2006), no. 4, 28-39.

[dCRC $\left.{ }^{+} 12\right]$ Irene del Campo, Raúl Ruiz, Ana Cuevas, Carlos Revilla, Luis Vielva, and Fernando de la Cruz, Determination of conjugation rates on solid surfaces, Plasmid 67 (2012), no. 2, 174-182.

[DFW73] J. N. Dabes, R. K. Finn, and C. R. Wilke, Equations of substratelimited growth: The case for blackman kinetics, Biotechnology and Bioengineering 15 (1973), no. 6, 1159-1177.

[DG97] M. Dorigo and L.M. Gambardella, Ant colony system: a cooperative learning approach to the traveling salesman problem, IEEE Transactions on Evolutionary Computation 1 (1997), no. 1, 53-66.

[DLM00] U Dieckmann, R Law, and J A J Metz, The Geometry of Ecological Interactions, vol. 1, 2000.

[Du 13] Andrea Du Toit, Cytoskeleton: Remodelling the FtsZ network, Nature Reviews Molecular Cell Biology 15 (2013), no. 1, 3-3. 
[DVS83] A. Depicker, M. Van Montagu, and J. Schell, Plant Cell Transformation by Agrobacterium Plasmids, Genetic Engineering of Plants, Springer US, Boston, MA, 1983, pp. 143-176.

[EGJ ${ }^{+}$13] Matthew R Evans, Volker Grimm, Karin Johst, Tarja Knuuttila, et al., Do simple models lead to generality in ecology?, Trends in ecology \& evolution 28 (2013), no. 10, 578-83.

[EMN ${ }^{+}$05] T. Emonet, C. M. Macal, M. J. North, C. E. Wickersham, and P. Cluzel, AgentCell: a digital single-cell assay for bacterial chemotaxis, Bioinformatics 21 (2005), no. 11, 2714-2721.

[ESJ07] Štefan Emrich, Sergej Suslov, and Florian Judex, Fully agent based modellings of epidemic spread using Anylogic, Proceeding EUROSIM 2007, Ljubljana, Slovenia 2007 (2007), 1-7.

[Far93] Stephen K. Farrand, Conjugal Transfer of Agrobacterium Plasmids, Bacterial Conjugation, Springer US, Boston, MA, 1993, pp. 255-291.

[FKJC14] Peter D Freese, Kirill S Korolev, José I Jiménez, and Irene A Chen, Genetic drift suppresses bacterial conjugation in spatially structured populations., Biophysical journal 106 (2014), no. 4, 944-54.

[FLdCR ${ }^{+}$14] Raul Fernandez-Lopez, Irene del Campo, Carlos Revilla, Ana Cuevas, and Fernando de la Cruz, Negative Feedback and Transcriptional Overshooting in a Regulatory Network for Horizontal Gene Transfer, PLoS Genetics 10 (2014), no. 2, e1004171.

[FPL08] Jordi Ferrer, Clara Prats, and Daniel López, Individual-based modelling: an essential tool for microbiology., Journal of biological physics 34 (2008), no. 1-2, 19-37.

[Fre90] Moselio Schaechter Frederick C. Neidhardt, John L. Ingraham, Physiology of the Bacterial Cell: A Molecular Approach, 1 ed., Sunderland, Mass. : Sinauer Associates, Sunderland Mass., 1990. 
[FZKT08] Randal E Fox, Xue Zhong, Stephen M Krone, and Eva M Top, Spatial structure and nutrients promote invasion of IncP-1 plasmids in bacterial populations, The ISME Journal 2 (2008), no. 10, 1024-1039.

[Gar13] Aurora Sofía Arroyo García, Enhancing idynomics framework with a plasmid conjugation module, Master's thesis, Facultad de informática(UPM), 2013.

[GAR16] Volker Grimm, Daniel Ayllón, and Steven F. Railsback, NextGeneration Individual-Based Models Integrate Biodiversity and Ecosystems: Yes We Can, and Yes We Must, Ecosystems (2016), $1-8$.

[GBB ${ }^{+}$06] Volker Grimm, Uta Berger, Finn Bastiansen, Sigrunn Eliassen, Vincent Ginot, Jarl Giske, John Goss-Custard, Tamara Grand, Simone K. Heinz, Geir Huse, Andreas Huth, Jane U. Jepsen, Christian Jørgensen, Wolf M. Mooij, Birgit Müller, Guy Pe'er, Cyril Piou, Steven F. Railsback, Andrew M. Robbins, Martha M. Robbins, Eva Rossmanith, Nadja Rüger, Espen Strand, Sami Souissi, Richard A. Stillman, Rune Vabø, Ute Visser, and Donald L. DeAngelis, A standard protocol for describing individual-based and agent-based models, Ecological Modelling 198 (2006), no. 1-2, 115-126.

[GBD+10] Volker Grimm, Uta Berger, Donald L. DeAngelis, J. Gary Polhill, Jarl Giske, and Steven F. Railsback, The ODD protocol: A review and first update, Ecological Modelling 221 (2010), no. 23, 2760-2768.

[GBdIC08] M. Pilar Garcillán-Barcia and Fernando de la Cruz, Why is entry exclusion an essential feature of conjugative plasmids?, Plasmid 60 (2008), no. 1, 1-18.

[GBP ${ }^{+}$15] Alexander Gutfraind, Basmattee Boodram, Nikhil Prachand, Atesmachew Hailegiorgis, Harel Dahari, and Marian E. Major, Agent-Based Model Forecasts Aging of the Population of People Who Inject Drugs in Metropolitan Chicago and Changing Prevalence of Hepatitis C Infections, PLOS ONE 10 (2015), no. 9, e0137993. 
[GGGP+17] Martín Gutiérrez, Paula Gregorio-Godoy, Guillermo Pérez del Pulgar, Luis E. Muñoz, Sandra Sáez, and Alfonso Rodríguez-Patón, A New Improved and Extended Version of the Multicell Bacterial Simulator gro, ACS Synthetic Biology (2017), acssynbio.7b00003.

[GH06] Arthur C Guyton and John E Hall, Textbook of Medical Physiology, vol. 51, 2006.

[Gio09] Toni Giorgino, Computing and Visualizing Dynamic Time Warping Alignments in R: The dtw Package, Journal Of Statistical Software 31 (2009), no. 7, 1-24.

[GLV02] Marta Ginovart, Daniel López, and Joaquim Valls, INDISIM, an individual-based discrete simulation model to study bacterial cultures., Journal of theoretical biology. 214 (2002), no. 2, 305-319.

[GM16] Erin R Green and Joan Mecsas, Bacterial Secretion Systems: An Overview., Microbiology spectrum 4 (2016), no. 1.

[GMA15] Angel Goñi-Moreno and Martyn Amos, DiSCUS: A Simulation Platform for Conjugation Computing, Springer, Cham, 2015, pp. 181-191.

[GMT+12] Thomas E. Gorochowski, Antoni Matyjaszkiewicz, Thomas Todd, Neeraj Oak, Kira Kowalska, Stephen Reid, Krasimira T. TsanevaAtanasova, Nigel J. Savery, Claire S. Grierson, and Mario di Bernardo, BSim: An Agent-Based Tool for Modeling Bacterial Populations in Systems and Synthetic Biology, PLoS ONE 7 (2012), no. 8.

[GR05a] V Grimm and Sf Railsback, Individual-based Modeling and Ecology, Individual-based Modeling and Ecology (2005), 1-432.

[GR05b] Volker Grimm and Steven F. Railsback, Individual-based Modeling and Ecology: (Princeton Series in Theoretical and Computational Biology), Princeton University Press, Princeton, July 2005.

[Hae96] James W. Haefner, Modeling biological systems : principles and applications, Springer-Verlag, New York, 1996. 
[Har60] G. Hardin, The Competitive Exclusion Principle, Science 131 (1960), no. 3409, 1292-1297.

[Hat08] Graham F Hatfull, Bacteriophage genomics., Current opinion in microbiology 11 (2008), no. 5, 447-53.

[HB09] Ferdi L. Hellweger and Vanni Bucci, A bunch of tiny individuals Individual-based modeling for microbes, Ecological Modelling 220 (2009), no. 1, 8-22.

[HB10] P. Horvath and R. Barrangou, CRISPR/Cas, the Immune System of Bacteria and Archaea, Science 327 (2010), no. 5962, 167-170.

[HB12] Ellie Harrison and Michael A Brockhurst, Plasmid-mediated horizontal gene transfer is a coevolutionary process., Trends in microbiology $\mathbf{2 0}$ (2012), no. 6, 262-7.

[HCD ${ }^{+95]}$ J. Helly, T. Case, F. Davis, S. Levin, and W. Michener, The State of Computational Ecology, San Diego, CA: San Diego Super Computer Center (1995).

[HCN ${ }^{+}$16] Clyde A. Hutchison, Ray-Yuan Chuang, Vladimir N. Noskov, Nacyra Assad-Garcia, Thomas J. Deerinck, Mark H. Ellisman, John Gill, Krishna Kannan, Bogumil J. Karas, Li Ma, James F. Pelletier, ZhiQing Qi, R. Alexander Richter, Elizabeth A. Strychalski, Lijie Sun, Yo Suzuki, Billyana Tsvetanova, Kim S. Wise, Hamilton O. Smith, John I. Glass, Chuck Merryman, Daniel G. Gibson, and J. Craig Venter, Design and synthesis of a minimal bacterial genome, Science 351 (2016), no. 6280.

[HGB ${ }^{+}$02] Arlene R. Howe, Charles S. Gasser, Sherri M. Brown, Stephen R. Padgette, Jesse Hart, Gregory B. Parker, Michael E. Fromm, and Charles L. Armstrong, Glyphosate as a selective agent for the production of fertile transgenic maize (Zea mays L.) plants, Molecular Breeding 10 (2002), no. 3, 153-164. 
[HH16] James P. J. Hall and Ellie Harrison, Bacterial evolution: Resistance is a numbers game, Nature Microbiology 1 (2016), 16235.

[Hic93] Charles R. Hicks, Fundamental Concepts in the Design of Experiments, 4 ed., Oxford University Press, USA, March 1993.

[HKB06] Kathrin Happe, Konrad Kellermann, and Alfons Balmann, Agentbased analysis of agricultural policies: An illustration of the agriculutural policy simulator AgriPolis, its adaptation and behavior, Ecology and Society 11 (2006), no. 1.

[HKRW13] J. D. Herman, J. B. Kollat, P. M. Reed, and T. Wagener, Technical Note: Method of Morris effectively reduces the computational demands of global sensitivity analysis for distributed watershed models, Hydrology and Earth System Sciences 17 (2013), no. 7, 2893-2903.

[HMO ${ }^{+}$01] T. Hayashi, Kozo Makino, Makoto Ohnishi, Ken Kurokawa, Kazuo Ishii, Katsushi Yokoyama, Chang-Gyun Han, Eiichi Ohtsubo, Keisuke Nakayama, Takahiro Murata, Masashi Tanaka, Toru Tobe, Tetsuya lida, Hideto Takami, Takeshi Honda, Chihiro Sasakawa, Naotake Ogasawara, Teruo Yasunaga, Satoru Kuhara, Tadayoshi Shiba, Masahira Hattori, and Hideo Shinagawa, Complete Genome Sequence of Enterohemorrhagic Eschelichia coli O157:H7 and Genomic Comparison with a Laboratory Strain K-12, DNA Research 8 (2001), no. 1, $11-22$.

[Hog13] Stuart. Hogg, Essential microbiology, Wiley, 2013.

[JDC03] R. Jin, X. Du, and W. Chen, The use of metamodeling techniques for optimization under uncertainty, Structural and Multidisciplinary Optimization 25 (2003), no. 2, 99-116.

[JOEK12] Seunghee S. Jang, Kevin T. Oishi, Robert G. Egbert, and Eric Klavins, Specification and Simulation of Synthetic Multicelled Behaviors, ACS Synthetic Biology 1 (2012), no. 8, 365-374. 
[JRZC14] M. Juhas, D. R. Reuss, B. Zhu, and F. M. Commichau, Bacillus subtilis and Escherichia coli essential genes and minimal cell factories after one decade of genome engineering, Microbiology 160 (2014), no. Pt_$11,2341-2351$.

[JSMS11] Hongyuan Jiang, Fangwei Si, William Margolin, and Sean X Sun, Mechanical control of bacterial cell shape., Biophysical journal 101 (2011), no. 2, 327-35.

[JY13] Momin Jamil and Xin She Yang, A literature survey of benchmark functions for global optimisation problems, International Journal of Mathematical Modelling and Numerical Optimisation 4 (2013), no. 2, 150.

[KBW98] Jan-Ulrich Kreft, Ginger Booth, and Julian W. T. Wimpenny, BacSim, a simulator for individual-based modelling of bacterial colony growth, Microbiology 144 (1998), no. 12, 3275-3287.

[KC10] Ahmad S. Khalil and James J. Collins, Synthetic biology: applications come of age, Nature Reviews Genetics 11 (2010), no. 5, 367-379.

[KE95] J Kennedy and R Eberhart, Particle swarm optimization, Neural Networks, 1995. Proceedings., IEEE International Conference on $\mathbf{4}$ (1995), 1942-1948 vol.4.

[Kea08] Jay D. Keasling, Synthetic Biology for Synthetic Chemistry, ACS Chemical Biology 3 (2008), no. 1, 64-76.

[KG00] Matt J Keeling and Bryan T Grenfell, Individual-based Perspectives on $R_{0}$, J. theor. Biol 203 (2000), 51-61.

[KGV83] S Kirkpatrick, C D Gelatt, and M P Vecchi, Optimization by Simulated Annealing, Science 220 (1983), no. 4598, pp. 671-680.

$\left[\mathrm{KLF}^{+}\right.$07] Stephen M. Krone, Ruinan Lu, Randal Fox, Haruo Suzuki, and Eva M. Top, Modelling the spatial dynamics of plasmid transfer and persistence, Microbiology 153 (2007), no. 8, 2803-2816. 
[Koc90] Arthur L Koch, Growth and Form of the Bacterial Cell Wall, American Scientist 78 (1990), no. 4, 327-341.

[KPWvL01] Jan-Ulrich Kreft, Cristian Picioreanu, Julian W. T. Wimpenny, and Mark C. M. van Loosdrecht, Individual-based modelling of biofilms, Microbiology 147 (2001), no. 11, 2897-2912.

[KSM ${ }^{+}$12] Jonathan R. Karr, Jayodita C. Sanghvi, Derek N. Macklin, Miriam V. Gutschow, Jared M. Jacobs, Benjamin Bolival, Nacyra Assad-Garcia, John I. Glass, and Markus W. Covert, A Whole-Cell Computational Model Predicts Phenotype from Genotype, Cell 150 (2012), no. 2, 389-401.

[LA15] Petra Anne Levin and Esther R Angert, Small but Mighty: Cell Size and Bacteria., Cold Spring Harbor perspectives in biology 7 (2015), no. 7, a019216.

[Law05] Averill M. Law, How to build valid and credible simulation models, Proceedings of the 2005 Winter Simulation Conference (M. E. Kuhl, N. M. Steiger, F. B. Armstrong, and J. A. Joines, eds.), 2005, pp. 24-32.

[LCRP ${ }^{+}$05] Sean Luke, Claudio Cioffi-Revilla, Liviu Panait, Keith Sullivan, and Gabriel C. Balan, MASON: A Multiagent Simulation Environment, Simulation 81 (2005), no. 7, 517-527.

[LCWF05] Curtis M. Lively, Keith Clay, Michael J. Wade, and Clay Fuqua, Competitive co-existence of vertically and horizontally transmitted parasites, Evolutionary Ecology Research 7 (2005), no. 8, 1183-1190.

[Led52] J Lederberg, Cell genetics and hereditary symbiosis., Physiological reviews 32 (1952), no. 4, 403-430.

[LEV79] B LEVIN, The kinetics of conjugative plasmid transmission: Fit of a simple mass action model, Plasmid 2 (1979), no. 2, 247-260.

[LFLZ+15] Ju-Sung Lee, Tatiana Filatova, Arika Ligmann-Zielinska, Behrooz Hassani-Mahmooei, Forrest Stonedahl, Iris Lorscheid, Alexey Voinov, 
J. Gary Polhill, Zhanli Sun, and Dawn C. Parker, The Complexities of Agent-Based Modeling Output Analysis, Journal of Artificial Societies and Social Simulation 18 (2015), no. 4, 4.

[LH78] Thomas M. (Thomas Morton) Little and Frederick Jackson Hills, Agricultural experimentation : design and analysis, Wiley, 1978.

[LHM12] Iris Lorscheid, Bernd-Oliver Heine, and Matthias Meyer, Opening the 'black box' of simulations: increased transparency and effective communication through the systematic design of experiments, Computational and Mathematical Organization Theory 18 (2012), no. 1, 22-62.

[LKGF03a] T D Lawley, W A Klimke, M J Gubbins, and L S Frost, F factor conjugation is a true type IV secretion system., FEMS microbiology letters 224 (2003), no. 1, 1-15.

[LKGF03b] T. D. Lawley, W. A. Klimke, M. J. Gubbins, and L. S. Frost, F factor conjugation is a true type IV secretion system., FEMS microbiology letters 224 (2003), no. 1, 1-15.

[LKKP98] M Loferer-Krössbacher, J Klima, and R Psenner, Determination of bacterial cell dry mass by transmission electron microscopy and densitometric image analysis., Applied and environmental microbiology 64 (1998), no. 2, 688-94.

[LL86] Peter D. Lundquist and Bruce R. Levin, TRANSITORY DEREPRESSION AND THE MAINTENANCE OF CONJUGATIVE PLASMIDS, Genetics 113 (1986), no. 3, 483-497.

[LLS $\left.{ }^{+} 84\right] \mathrm{K}$ Lind, B O Lindhardt, H J Schütten, J Blom, and C Christiansen, Serological cross-reactions between Mycoplasma genitalium and Mycoplasma pneumoniae., Journal of clinical microbiology 20 (1984), no. 6 , 1036-43.

[LM15] Michael Lynch and Georgi K. Marinov, The bioenergetic costs of a gene, Proceedings of the National Academy of Sciences 112 (2015), no. $51,201514974$. 
[LMM $\left.{ }^{+} 11\right]$ Laurent A. Lardon, Brian V. Merkey, Sónia Martins, Andreas Dötsch, Cristian Picioreanu, Jan Ulrich Kreft, and Barth F. Smets, iDynoMiCS: Next-generation individual-based modelling of biofilms, Environmental Microbiology 13 (2011), no. 9, 2416-2434.

[LNEM95] M. Lipsitch, M. A. Nowak, D. Ebert, and R. M. May, The Population Dynamics of Vertically and Horizontally Transmitted Parasites, Proceedings of the Royal Society B: Biological Sciences 260 (1995), no. $1359,321-327$.

[Loh96] Wei-Liem Loh, On Latin hypercube sampling, The Annals of Statistics 24 (1996), no. 5, 2058-2080.

[LT46] J Lederberg and E L Tatum, Gene recombination in Escherichia coli., 1946, p. 558.

[LWGP03] Cristina Lagido, Ian J Wilson, L.Anne Glover, and Jim I Prosser, A model for bacterial conjugal gene transfer on solid surfaces, FEMS Microbiology Ecology 44 (2003), no. 1, 67-78.

[MCO05] Daniela Morale, Vincenzo Capasso, and Karl Oelschläger, An interacting particle system modelling aggregation behavior: from individuals to populations, Journal of Mathematical Biology 50 (2005), no. 1, 49-66.

[Min65] Marvin Minsky, Matter, Mind and Models, jan 1965, pp. 45-49.

[MLS+11] Brian V. Merkey, Laurent A. Lardon, Jose M. Seoane, Jan-Ulrich Kreft, and Barth F. Smets, Growth dependence of conjugation explains limited plasmid invasion in biofilms: an individual-based modelling study, Environmental Microbiology 13 (2011), no. 9, 2435-2452.

[Mor91] Max D Morris, Factorial Sampling Plans for Preliminary Computational Experiments, Technometrics 33 (1991), no. 2, 161-174.

[MPN16] Ron Mil, Rob Phillips, and Orme Nigel, CELL BIOLOGY by the numbers, Garland Science, New York, New York, USA, 2016. 
[MW95] Jerome L. Myers and Arnold D. Well, Research Design \& Statistical Analysis, 1 ed., Routledge, June 1995.

[NCO ${ }^{+}$13a] Michael J North, Nicholson T Collier, Jonathan Ozik, Eric R Tatara, Charles M Macal, Mark Bragen, and Pam Sydelko, Complex adaptive systems modeling with Repast Simphony, Complex Adaptive Systems Modeling 1 (2013), no. 1, 3.

[NCO ${ }^{+}$13b] MichaelJ North, NicholsonT Collier, Jonathan Ozik, EricR Tatara, CharlesM Macal, Mark Bragen, and Pam Sydelko, Complex adaptive systems modeling with Repast Simphony, Complex Adaptive Systems Modeling 1 (2013), no. 1, 1-26.

[NCO13c] Mj North, Nt Collier, and Jonathan Ozik, Complex adaptive systems modeling with repast simphony, Complex adaptive ... (2013), 1-26.

[NCO ${ }^{+}$13d] Mj Michael J North, Nicholson T Nt Collier, Jonathan Ozik, Eric R Tatara, Charles M Macal, Mark Bragen, and Pam Sydelko, Complex adaptive systems modeling with Repast Simphony, Complex Adaptive Systems Modeling 1 (2013), no. 1, 1-26.

[Nes15] Eugene W. Nester, Agrobacterium: nature's genetic engineer, Frontiers in Plant Science 5 (2015), 730.

[NHS09] Anders Norman, Lars H. Hansen, and Søren J. Sørensen, Conjugative plasmids: vessels of the communal gene pool, Philosophical Transactions of the Royal Society B: Biological Sciences 364 (2009), no. 1527, 2275-2289.

[Pas05] Mercedes Pascual, Computational Ecology: From the Complex to the Simple and Back, PLoS Computational Biology 1 (2005), no. 2, e18.

[PBF $\left.{ }^{+} 16\right]$ Francesca Pianosi, Keith Beven, Jim Freer, Jim W. Hall, Jonathan Rougier, David B. Stephenson, and Thorsten Wagener, Sensitivity analysis of environmental models: A systematic review with practical workflow, Environmental Modelling \& Software 79 (2016), 214-232. 
[Pie04] Benjamin Pierce, Genetics: A Conceptual Approach, 2004.

[Pie12] Benjamin A. Pierce, Genetics: A Conceptual Approach, 4th Edition, fourth edit ed., W. H. Freeman, dec 2012.

[PIwcfSDV ${ }^{+}$15] Gilles Pujol, Bertrand looss, Alexandre Janon with contributions from Sebastien Da Veiga, Jana Fruth, Laurent Gilquin, Joseph Guillaume, Loic Le Gratiet, Paul Lemaitre, Bernardo Ramos, and Taieb Touati, sensitivity: Sensitivity analysis, 2015, R package version 1.11.1.

[PKB07] Riccardo Poli, James; Kennedy, and Tim Blackwell, Particle swarm optimization, Swarm Intelligence 1 (2007), no. 1, 33-57.

[PKTG12] Rob Phillips, Jane Kondev, Julie Theriot, and Hernan Garcia, Physical Biology of the Cell, 2 ed., Garland Science, London ; New York, NY, 2012.

$\left[\mathrm{PPH}^{+}\right.$12] Sergei Petrovskii, Natalia Petrovskaya, J. D. Hughes, et al., Computational ecology as an emerging science., Interface focus 2 (2012), no. 2, 241-54.

[Pre11] Antonio Prestes García, A first approach to individual-based modeling of the bacterial conjugation dynamics, Master's thesis, Facultad de informática(UPM), September 2011.

[PRP15a] Antonio Prestes García and Alfonso Rodríguez-Patón, A Preliminary Assessment of Three Strategies for the Agent-Based Modeling of Bacterial Conjugation, 9th International Conference on Practical Applications of Computational Biology and Bioinformatics (Ross Overbeek, Miguel P. Rocha, Florentino Fdez-Riverola, and Juan F. De Paz, eds.), Advances in Intelligent Systems and Computing, vol. 375, Springer International Publishing, 2015, pp. 1-9.

[PRP15b] Antonio Prestes García and Alfonso Rodríguez-Patón, BactoSim An Individual-Based Simulation Environment for Bacterial Conjugation, Springer International Publishing, 2015, pp. 275-279. 
[QLW+16] B.Y. Qu, J.J. Liang, Z.Y. Wang, Q. Chen, and P.N. Suganthan, Novel benchmark functions for continuous multimodal optimization with comparative results, Swarm and Evolutionary Computation 26 (2016), 23-34.

[R C15] R Core Team, R: A language and environment for statistical computing, R Foundation for Statistical Computing, Vienna, Austria, 2015.

[RARE ${ }^{+}$04] A. Rozkov, C. A. Avignone-Rossa, P. F. Ertl, P. Jones, R. D. O'Kennedy, J. J. Smith, J. W. Dale, and M. E. Bushell, Characterization of the metabolic burden on Escherichia coli DH1 cells imposed by the presence of a plasmid containing a gene therapy sequence., Biotechnology and bioengineering 88 (2004), no. 7, 909-915.

[RBH ${ }^{+}$83] S G Rogers, L A Brand, S B Holder, E S Sharps, and M J Brackin, Amplification of the aroA gene from Escherichia coli results in tolerance to the herbicide glyphosate., Applied and environmental microbiology 46 (1983), no. 1, 37-43.

[RVPM15] R. J. Roberts, T. Vincze, J. Posfai, and D. Macelis, REBASE-a database for DNA restriction and modification: enzymes, genes and genomes, Nucleic Acids Research 43 (2015), no. D1, D298-D299.

[RWZ12] Andreas Reisner, Heimo Wolinski, and Ellen L Zechner, In situ monitoring of IncF plasmid transfer on semi-solid agar surfaces reveals a limited invasion of plasmids in recipient colonies., Plasmid 67 (2012), no. $2,155-161$.

[Sal08] A Saltelli, Global Sensitivity Analysis: The Primer, International Statistical Review (2008), 452.

[SBH+ 05] Soren J. Sorensen, Mark Bailey, Lars H. Hansen, Niels Kroer, and Stefan Wuertz, Studying plasmid horizontal transfer in situ: a critical review, Nature Reviews Microbiology 3 (2005), no. 9, 700-710. 
[SBTT08] Frances R. Slater, Mark J. Bailey, Adrian J. Tett, and Sarah L. Turner, Progress towards understanding the fate of plasmids in bacterial communities, FEMS Microbiology Ecology 66 (2008), no. 1, 3-13.

[SD08] Krzysztof Socha and Marco Dorigo, Ant colony optimization for continuous domains, European Journal of Operational Research 185 (2008), no. 3, 1155-1173.

[SGSL90] L. Simonsen, D. M. Gordon, F. M. Stewart, and B. R. Levin, Estimating the rate of plasmid transfer: an end-point method, J Gen Microbiol 136 (1990), no. 11, 2319-2325.

[SHL] Davir R Setzer, Roberta M Hmiel, and Shaoyi Liao, A simple vector modification to facilitate oligonucleotide- directed mutagenesis, Nucleic Acids Research 18, no. 14.

[Sho08] Ronald W. Shonkwiler, Mathematical Biology: An Introduction with Maple and Matlab, 2nd ed., Springer Publishing Company, Incorporated, 2008.

[SHPC13] Larry Snyder, Tina M. Henkin, Joseph E. Peters, and Wendy Champness, Molecular Genetics of Bacteria, 4th Edition, Larry Snyder, Joseph E. Peters, Tina M. Henkin and Wendy Champness 1 (2013), 162-163, 503.

[SJPD07] Guennadi Sezonov, Danièle Joseleau-Petit, and Richard D'Ari, Escherichia coli physiology in Luria-Bertani broth., Journal of bacteriology 189 (2007), no. 23, 8746-9.

[SJS ${ }^{+11]}$ Hyun-Woo Shim, Yun-Ho Jin, Seung-Deok Seo, Seung-Hun Lee, and Dong-Wan Kim, Highly Reversible Lithium Storage in Bacillus subtilisDirected Porous Co3 O4 Nanostructures, ACS Nano 5 (2011), no. 1, 443-449.

[SKW10] Thomas J Silhavy, Daniel Kahne, and Suzanne Walker, The bacterial cell envelope., Cold Spring Harbor perspectives in biology 2 (2010), no. 5 , a000414. 
[SL77] FM Stewart and BR Levin, The Population Biology of Bacterial Plasmids: A PRIORI Conditions for the Existence of Conjugationally Transmitted Factors., Genetics 87 (1977), no. 2, 209-228.

[Smi74] John Maynard Smith, Models in ecology, revised ed ed., Cambridge University Press, 1974.

[Sör15] Kenneth Sörensen, Metaheuristics-the metaphor exposed, International Transactions in Operational Research 22 (2015), no. 1, 3-18.

[SRP $\left.{ }^{+} 13\right]$ Laura K. Sycuro, Chelsea S. Rule, Timothy W. Petersen, Timna J. Wyckoff, Tate Sessler, Dilip B. Nagarkar, Fakhra Khalid, Zachary Pincus, Jacoby Biboy, Waldemar Vollmer, and Nina R. Salama, Flow cytometry-based enrichment for cell shape mutants identifies multiple genes that influence $<\mathrm{i}>\mathrm{H}</ \mathrm{i}><\mathrm{i}>$ elicobacter pylori $<\mathrm{i}>$ morphology, Molecular Microbiology 90 (2013), no. 4, 869-883.

[ST16] Thibault Stalder and Eva Top, Plasmid transfer in biofilms: a perspective on limitations and opportunities, npj Biofilms and Microbiomes 2 (2016), 16022.

[STCR04] A. Saltelli, Stefano Tarantola, Francesca Campolongo, and Marco Ratto, Sensitivity Analysis in Practice: A Guide to Assessing Scientific Models, 1 ed., Wiley, April 2004.

[Str] Lubert Stryer, Biochemistry, 4th ed., W.H. Freeman \& Company.

[Sum96] David K. Summers, The Biology of Plasmids, Blackwell Publishing Ltd., Oxford, UK, mar 1996.

[SYD+11] Jose Seoane, Tatiana Yankelevich, Arnaud Dechesne, Brian Merkey, Claus Sternberg, and Barth F. Smets, An individual-based approach to explain plasmid invasion in bacterial populations, FEMS Microbiology Ecology 75 (2011), no. 1, 17-27.

[TKG14] Jan C. Thiele, Winfried Kurth, and Volker Grimm, Facilitating Parameter Estimation and Sensitivity Analysis of Agent-Based Models: A 
Bibliography

Cookbook Using NetLogo and 'R', Journal of Artificial Societies and Social Simulation 17 (2014), no. 3.

[TLNV15] Ignace L.M.M. Tack, Filip Logist, Estefanía Noriega Fernández, and Jan F.M. Van Impe, An individual-based modeling approach to simulate the effects of cellular nutrient competition on Escherichia coli K-12 MG1655 colony behavior and interactions in aerobic structured food systems, Food Microbiology 45 (2015), 179-188.

[TN05] Christopher M. Thomas and Kaare M. Nielsen, Mechanisms of, and Barriers to, Horizontal Gene Transfer between Bacteria, Nature Reviews Microbiology 3 (2005), no. 9, 711-721.

[TW04] Seth Tisue and Uri Wilensky, Netlogo: A simple environment for modeling complexity, ... Conference on Complex Systems (2004), 1-10.

[Urb16] Simon Urbanek, rjava: Low-level $r$ to java interface, 2016, $R$ package version 0.9-8.

[vHBC11] Hans C. van Houwelingen, Hendriek C Boshuizen, and Maurizio Capannesi, Sensitivity analysis of state-transition models: How to deal with a large number of inputs, Computers in Biology and Medicine $\mathbf{4 1}$ (2011), no. 9, 838-842.

[Via13] Felipe A C Viana, Things You Wanted to Know About the Latin Hypercube Design and Were Afraid to Ask, 10th World Congress on Structural and Multidisciplinary Optimization (2013), 1-9.

[Voe] Donald Voet, Biochemistry, 2nd Second Edition, water damaged, highlighted, notation ed., Wiley.

[Wei09] Thomas Weise, Global optimization algorithms - theory and application, self-published, Germany, 2009.

[WNF ${ }^{+}$15] A. Watkins, J. Noble, R.J. Foster, B.J. Harmsen, and C.P. Doncaster, A spatially explicit agent-based model of the interactions between jaguar populations and their habitats, Ecological Modelling 306 (2015), 268277. 
[WRS+14] J. Wilson White, Andrew Rassweiler, Jameal F. Samhouri, Adrian C. Stier, and Crow White, Ecologists should not use statistical significance tests to interpret simulation model results, Oikos 123 (2014), no. 4, 385-388.

[XG11] Chonggang Xu and George Gertner, Understanding and comparisons of different sampling approaches for the Fourier Amplitudes Sensitivity Test (FAST)., Computational statistics \& data analysis 55 (2011), no. 1, 184-198.

[You04] Kevin D. Young, Bacterial shape, Molecular Microbiology 49 (2004), no. $3,571-580$.

[You07] Kevin D Young, Bacterial morphology: why have different shapes?, Current opinion in microbiology 10 (2007), no. 6, 596-600.

[ZBCR13] Mauricio Zambrano-Bigiarini, Maurice Clerc, and Rodrigo Rojas, Standard Particle Swarm Optimisation 2011 at CEC-2013: A baseline for future PSO improvements, 2013 IEEE Congress on Evolutionary Computation, CEC 2013, 2013, pp. 2337-2344.

[ZDF ${ }^{+}$12] Xue Zhong, Jason Droesch, Randal Fox, Eva M. Top, and Stephen M. Krone, On the meaning and estimation of plasmid transfer rates for surface-associated and well-mixed bacterial populations, Journal of Theoretical Biology 294 (2012), 144-152.

[ZKaTK10] Xue Zhong, JarosLaw E. Kro[dot above]l, Eva M. Top, and Stephen M. Krone, Accounting for mating pair formation in plasmid population dynamics, Journal of Theoretical Biology 262 (2010), no. 4, 711-719.

[ZPK00] Bernard P. Zeigler, Herbert Praehofer, and Tag G. Kim, Theory of Modeling and Simulation, Second Edition, 2 ed., Academic Press, January 2000.

[ZR06] Y Zhang and A Rundell, Comparative study of parameter sensitivity analyses of the TCR-activated Erk-MAPK signalling pathway., Systems biology 153 (2006), no. 4, 201-11. 
[ZWV11] Arieh Zaritsky, Ping Wang, and Norbert O. Vischer, Instructive simulation of the bacterial cell division cycle., Microbiology (Reading, England) 157 (2011), no. Pt 7, 1876-1885. 
Appendix $\mathbf{A}$

Scripts for model analysis 
The objective of this Appendix is to ensure the reproducibility for the results contained in this work. Bearing this in mind, it is provided here all the scripts which are required in order to reproduce the experimental designs presented in this thesis. The scripts were developed using the GNU R language [R C15], [Cra07], requiring for their execution the installation of a version of FNU $R$ software environment for statistical computing which is freely available for download. Additionally, it will be necessary to install the two $\mathrm{R}$ packages developed in the scope of this work, namely the $\mathrm{R} /$ Repast and EvoPER which are both freely available from CRAN repository. Finally, it is also necessary to download and install the BactoSIM simulation software which is available for being downloaded from the following link https://goo.gl/aomr9G. It is important to take into account that for running these scripts without modification, the BactoSIM must be installed under the directory /usr/models/BactoSim(HaldaneEngine-1.0) otherwise it will be necessary to modify the installdir variable in all scripts. 


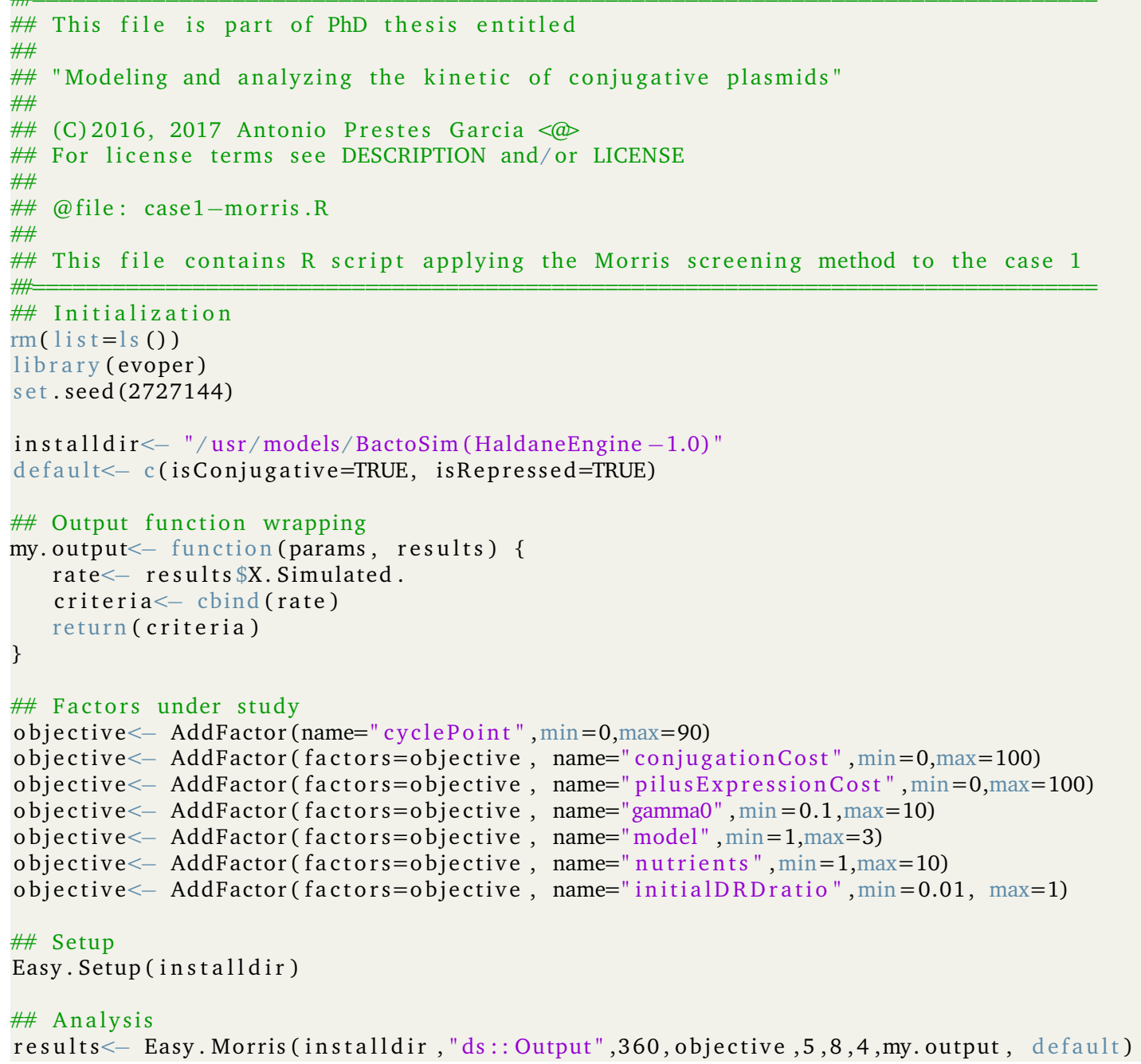

Figure A.1: The script for preliminary screening of case 1 of BactoSIM output analysis. 


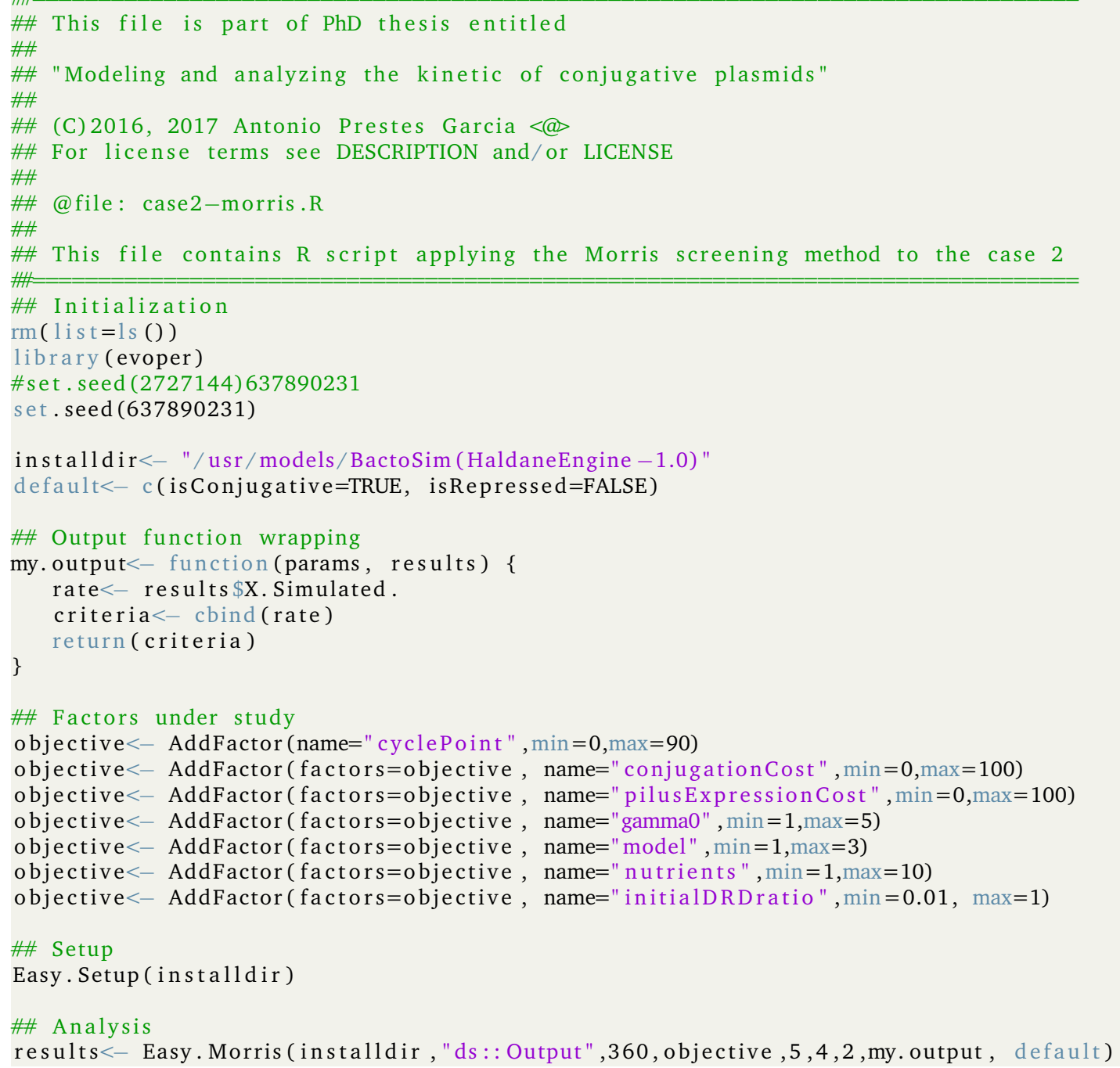

Figure A.2: The script for preliminary screening of case 2 of BactoSIM output analysis. 


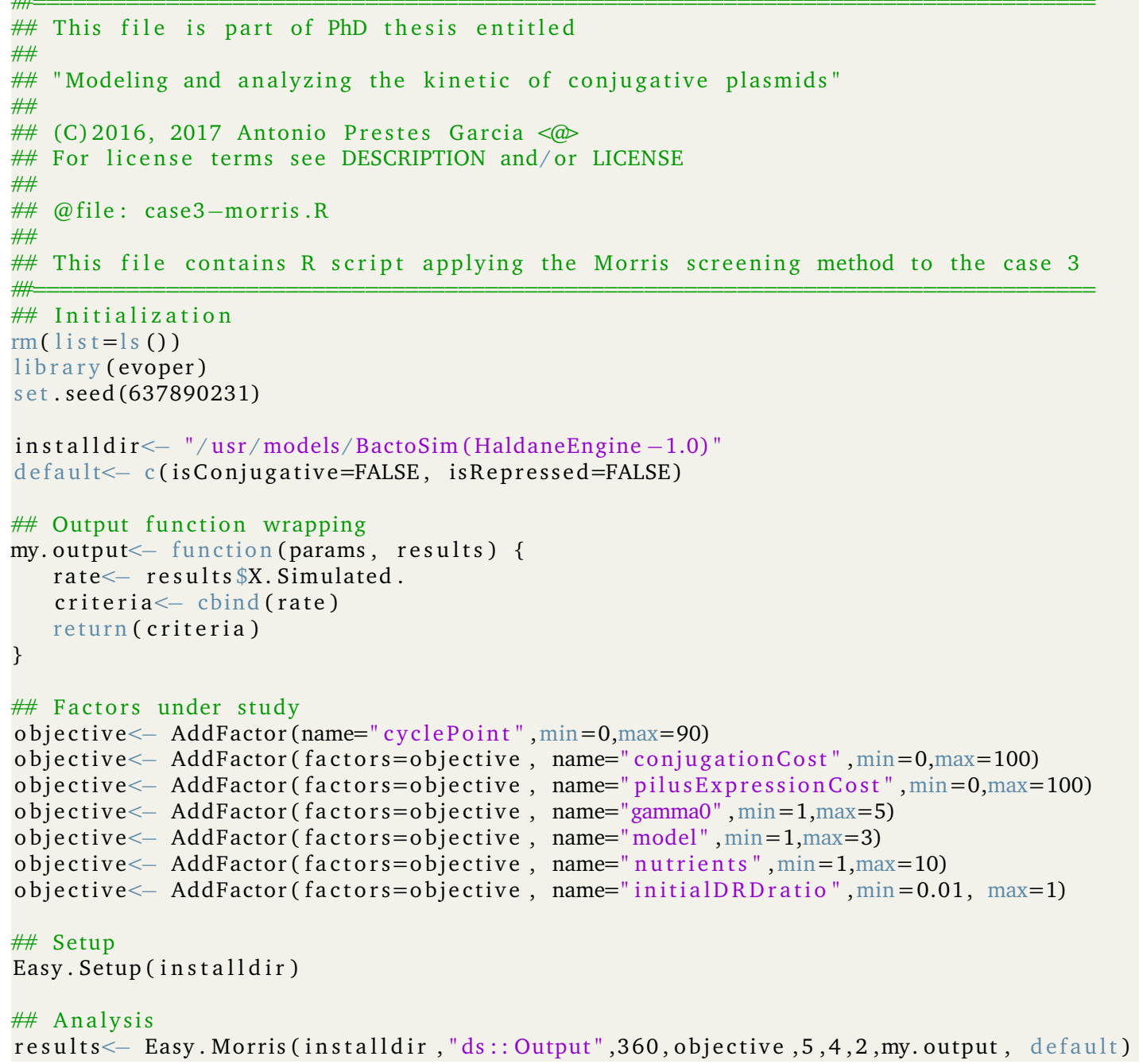

Figure A.3: The script for preliminary screening of case 3 of BactoSIM output analysis. 


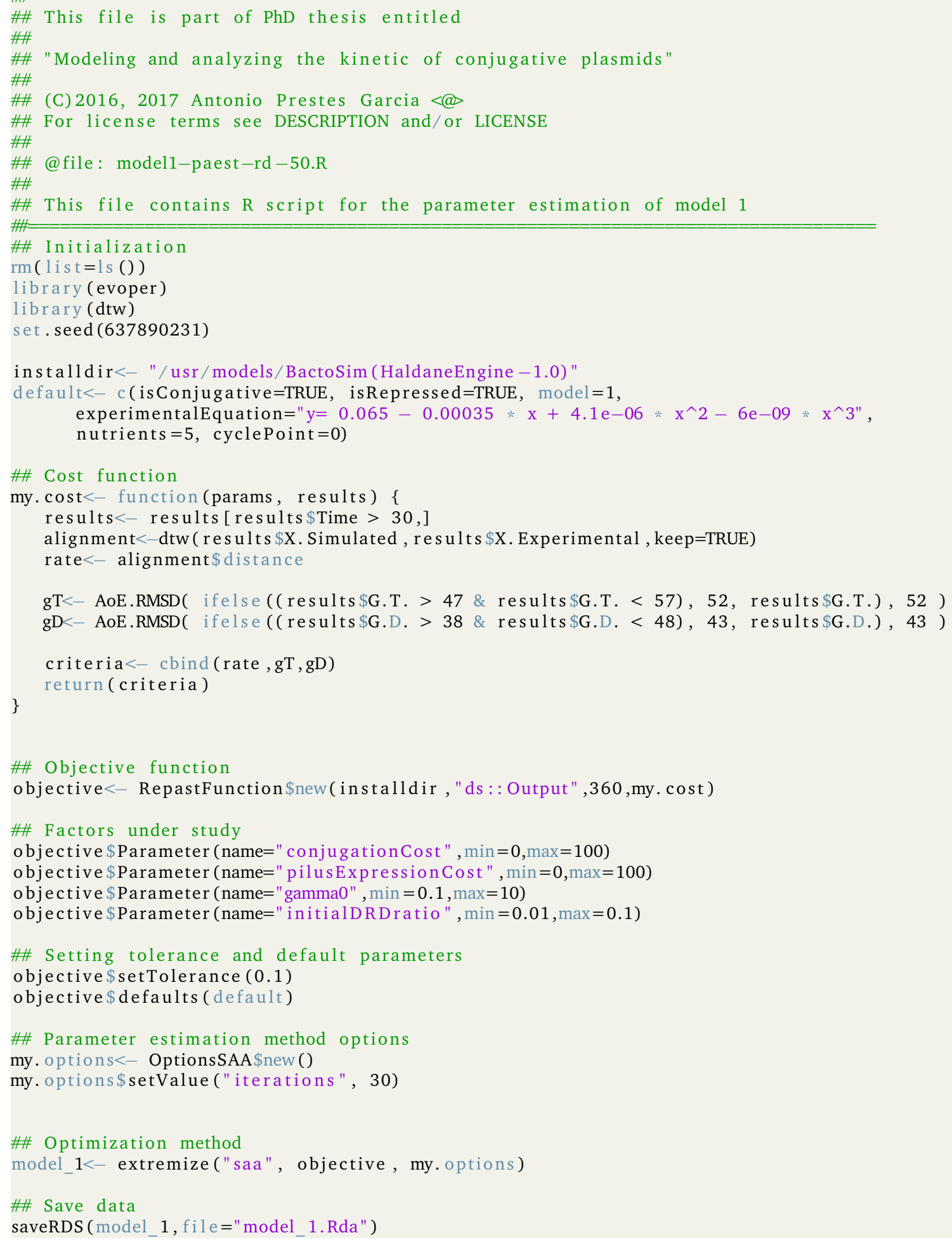

Figure A.4: The script for preliminary parameter estimation of model 1 of BactoSIM. 


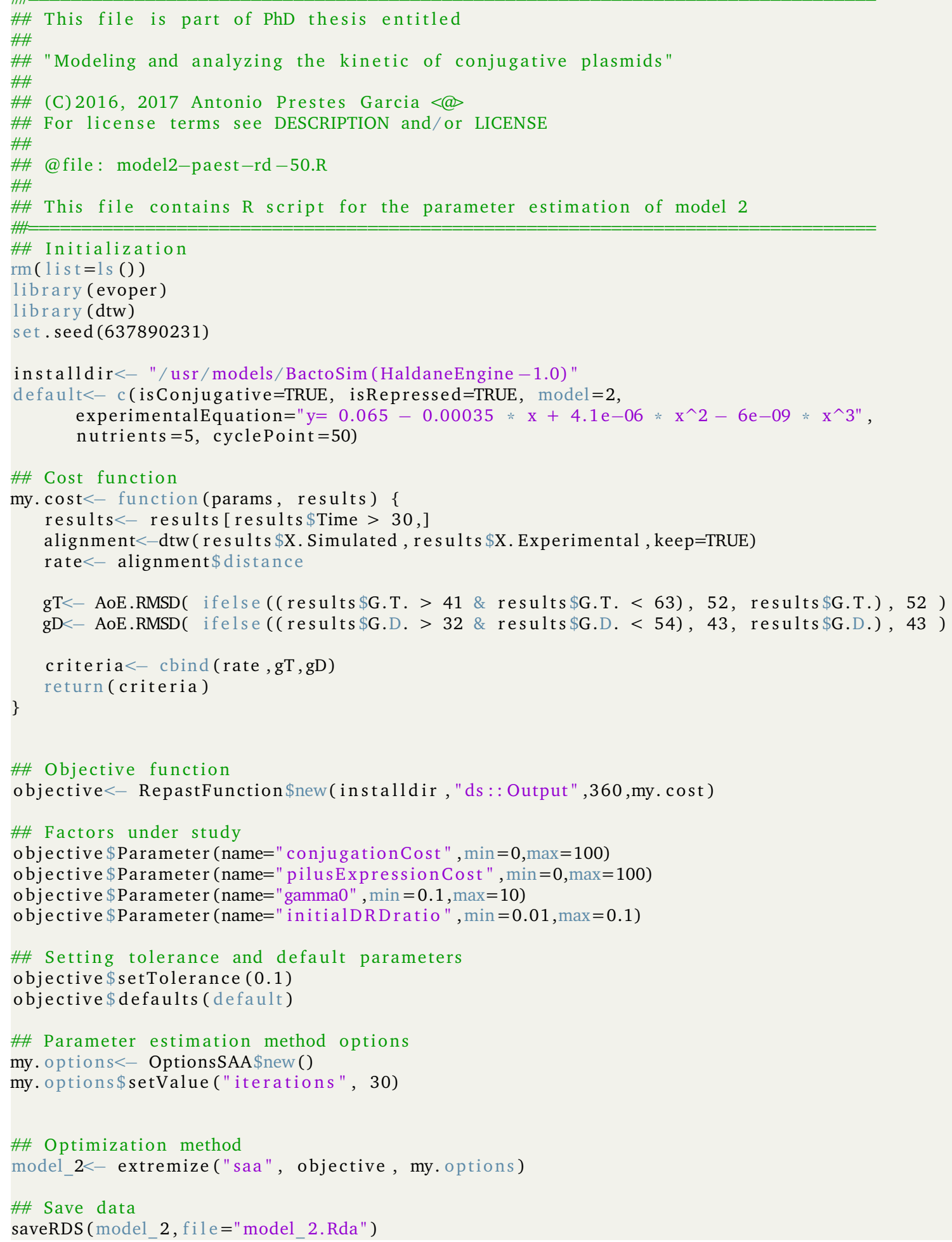

Figure A.5: The script for preliminary parameter estimation of model 2 of BactoSIM. 


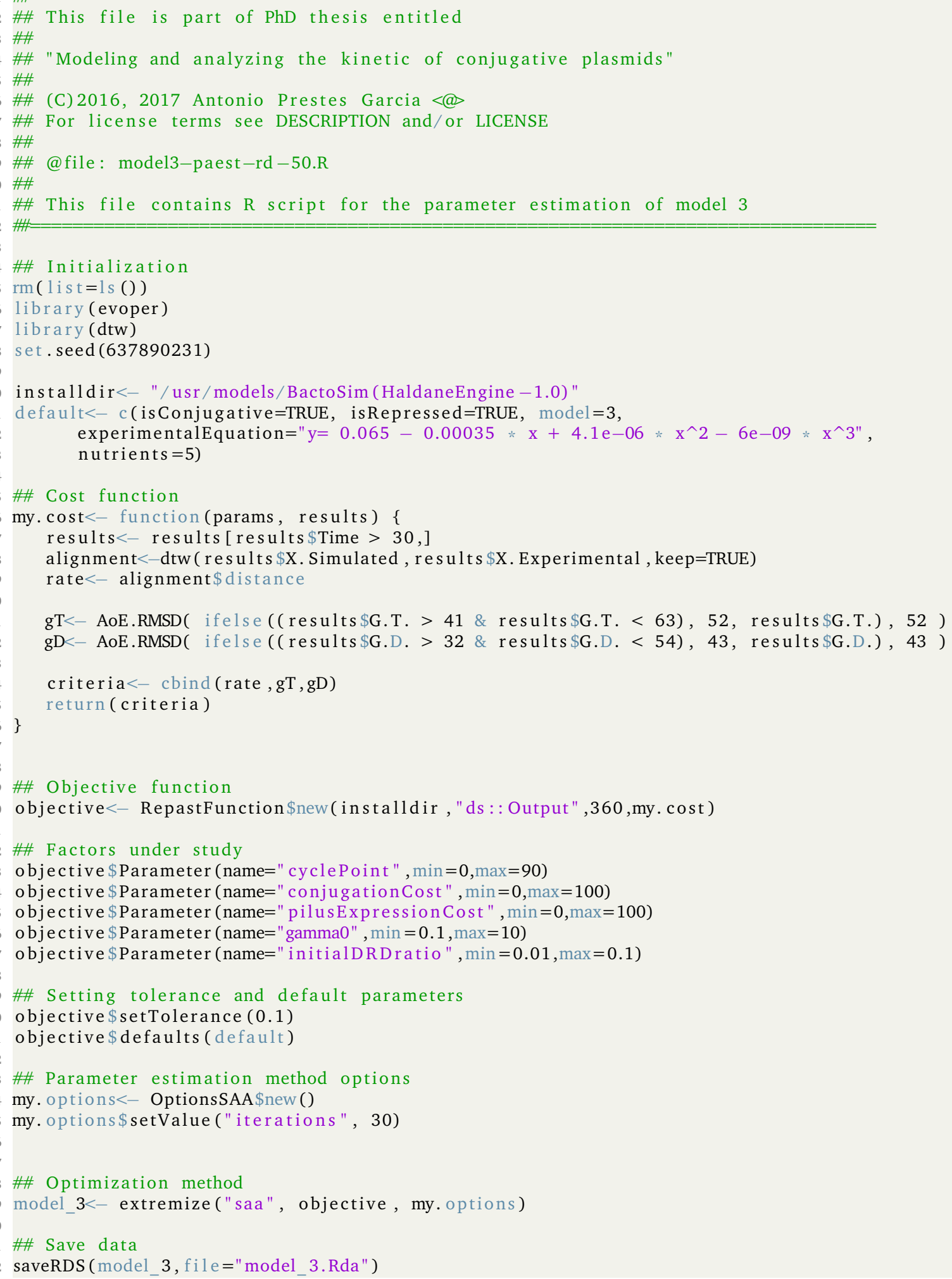

Figure A.6: The script for preliminary parameter estimation of model 3 of BactoSIM. 


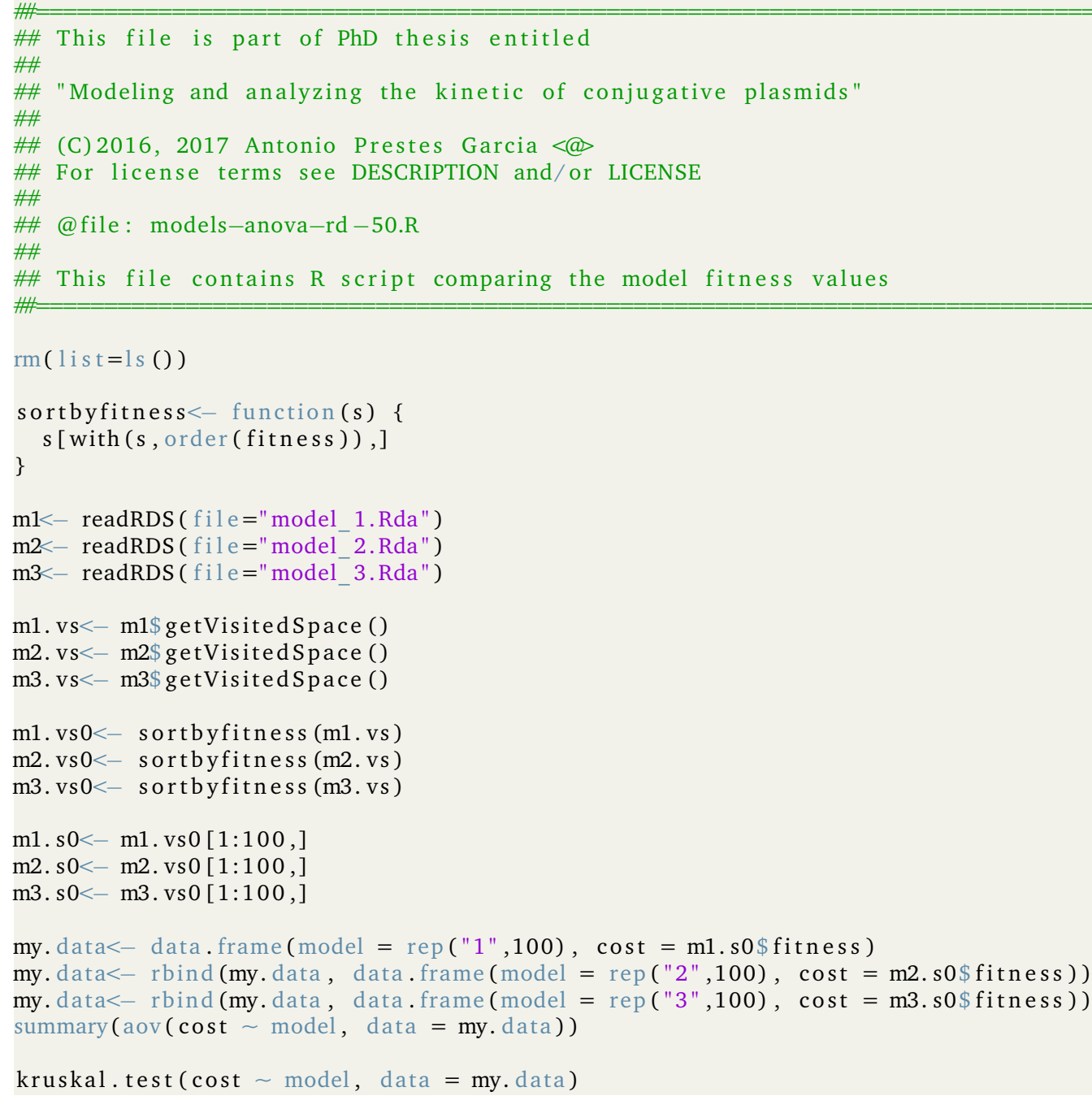

Figure A.7: The analysis of BactoSIM model type cost. 


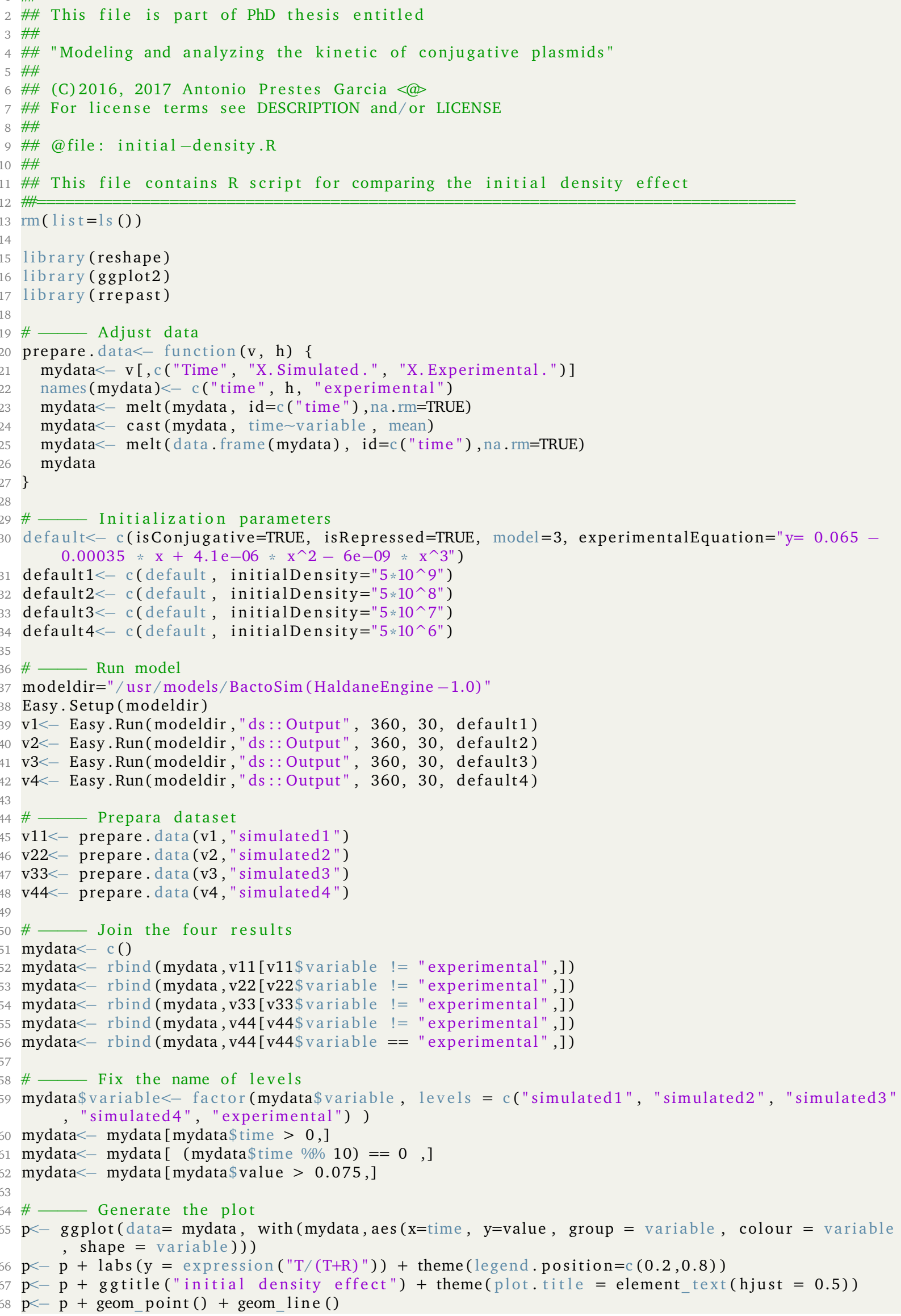

Figure A.8: The effect of initial cell density 


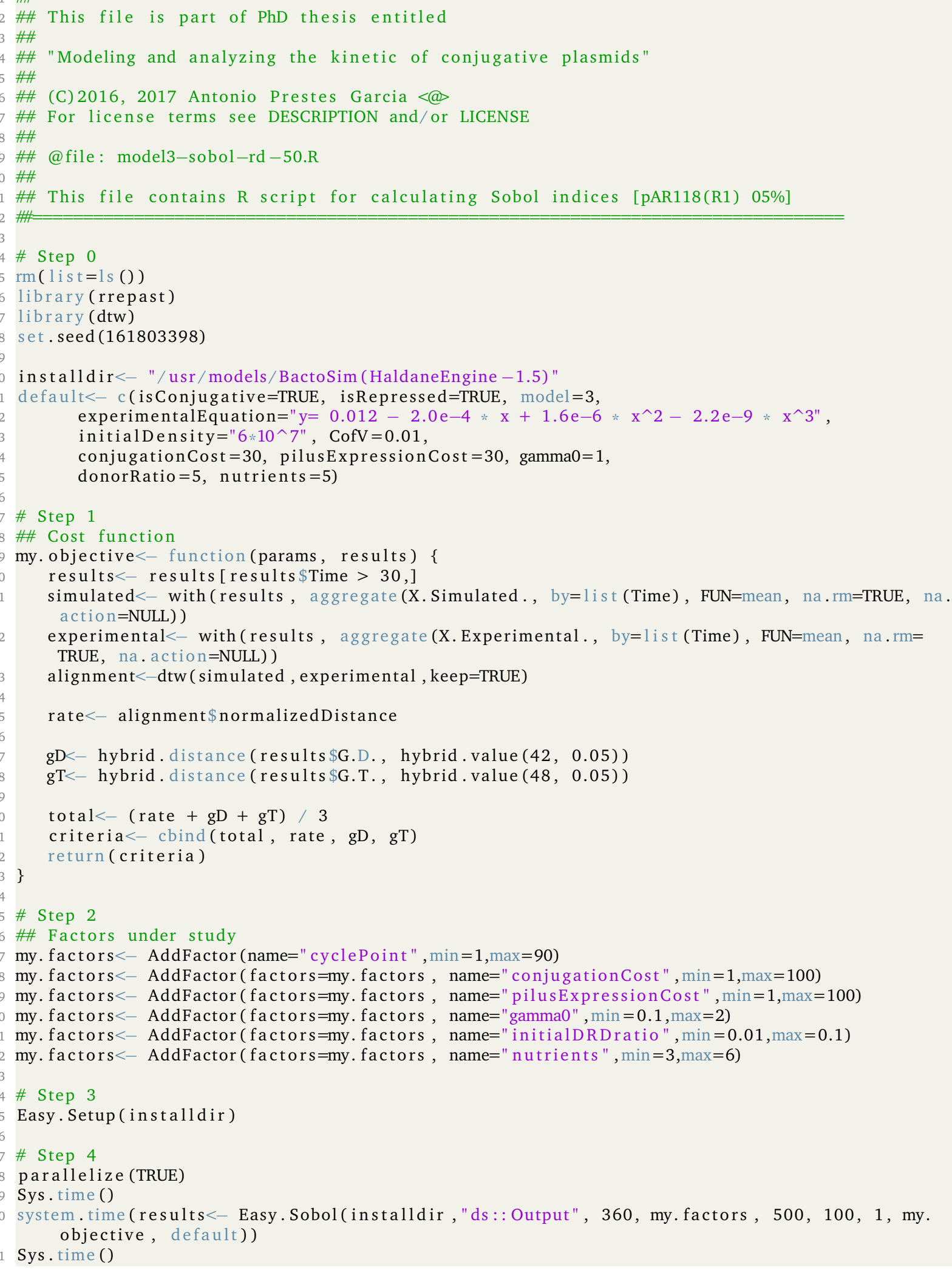

Figure A.9: The R code for applying Sobol GSA to pAR118/5\%. 


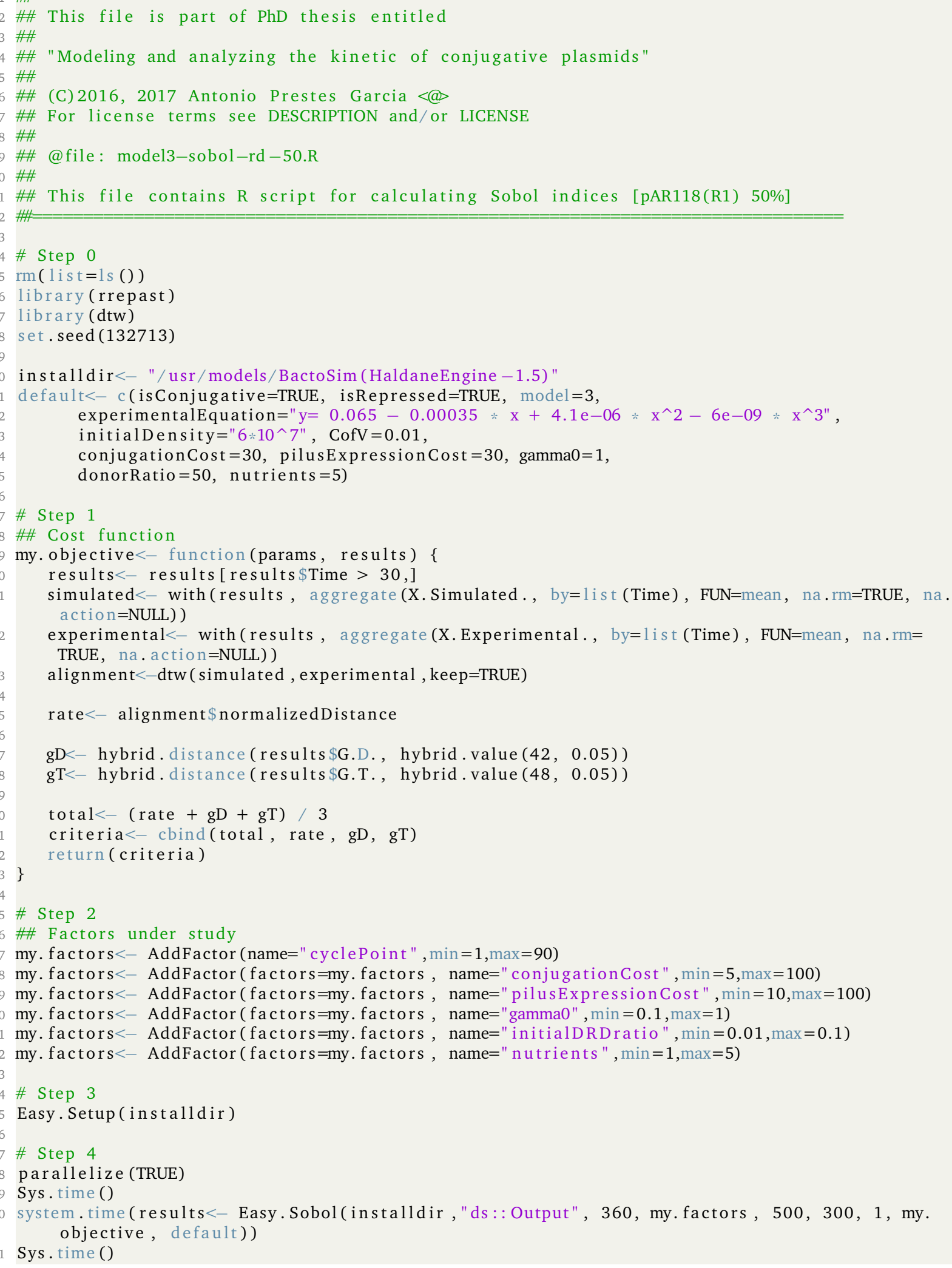

Figure A.10: The R code for applying Sobol GSA to pAR118/50\%. 


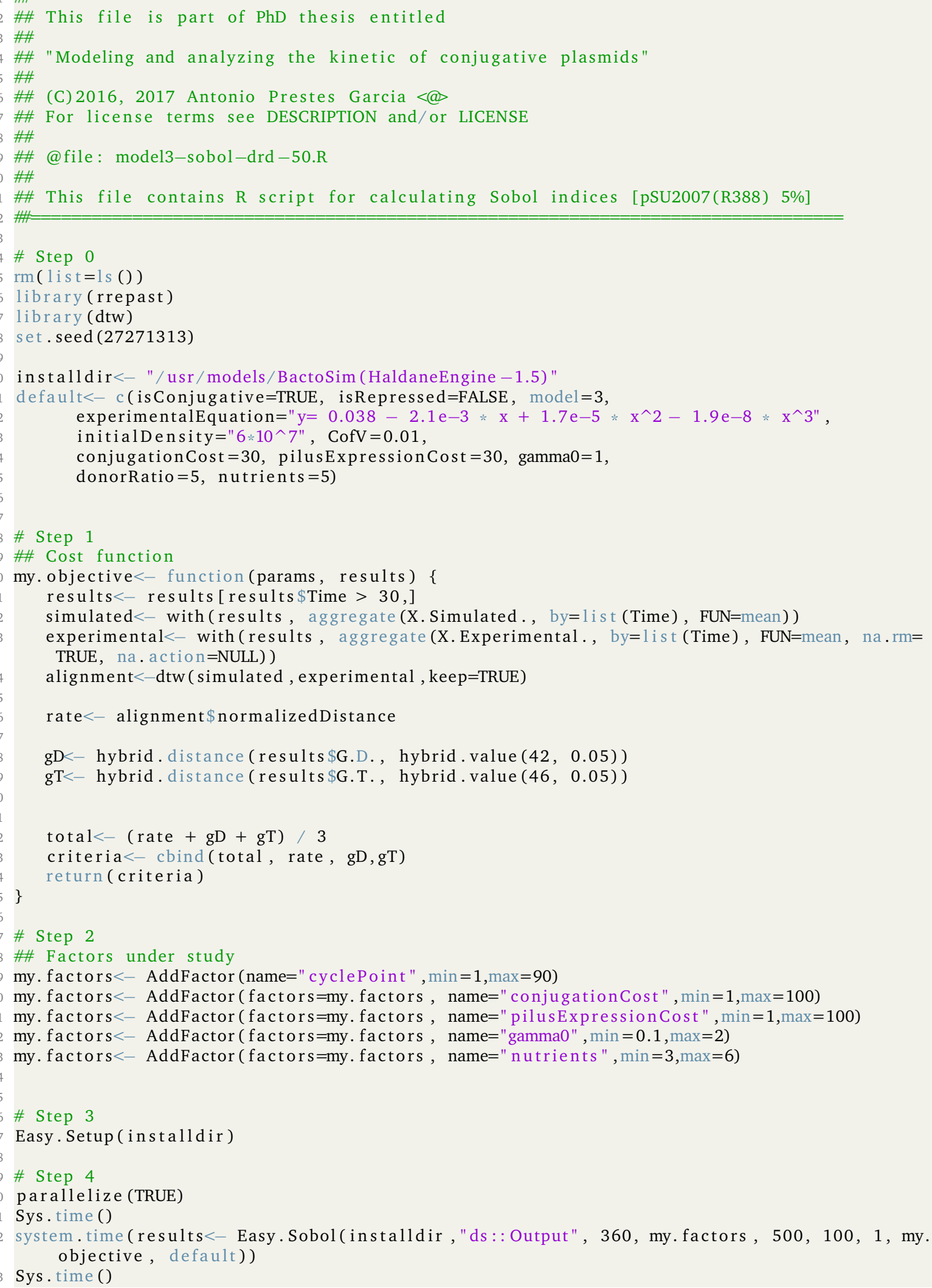

Figure A.11: The R code for applying Sobol GSA to pPSU2007/5\%. 


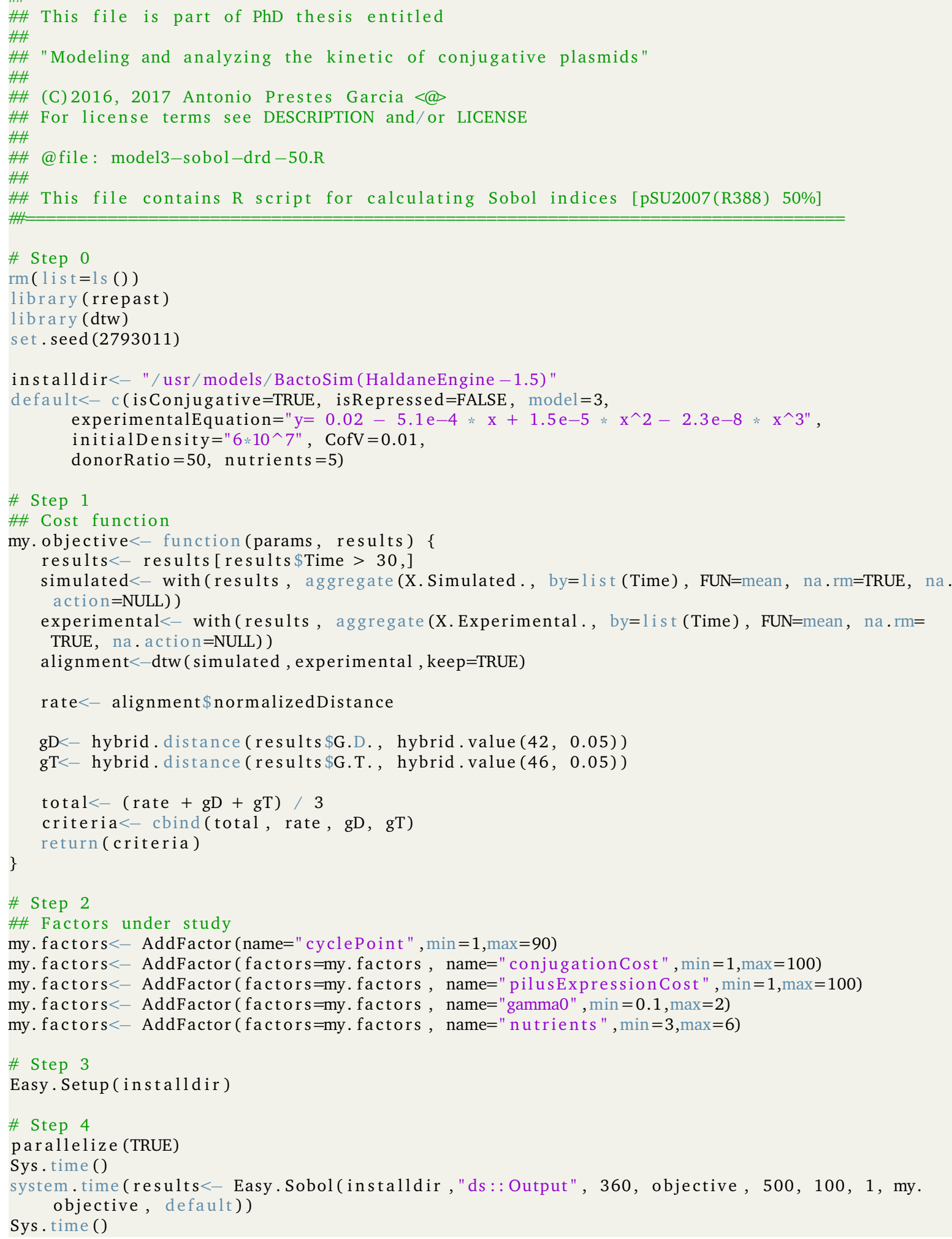

Figure A.12: The R code for applying Sobol GSA to pSU2007/50\%. 


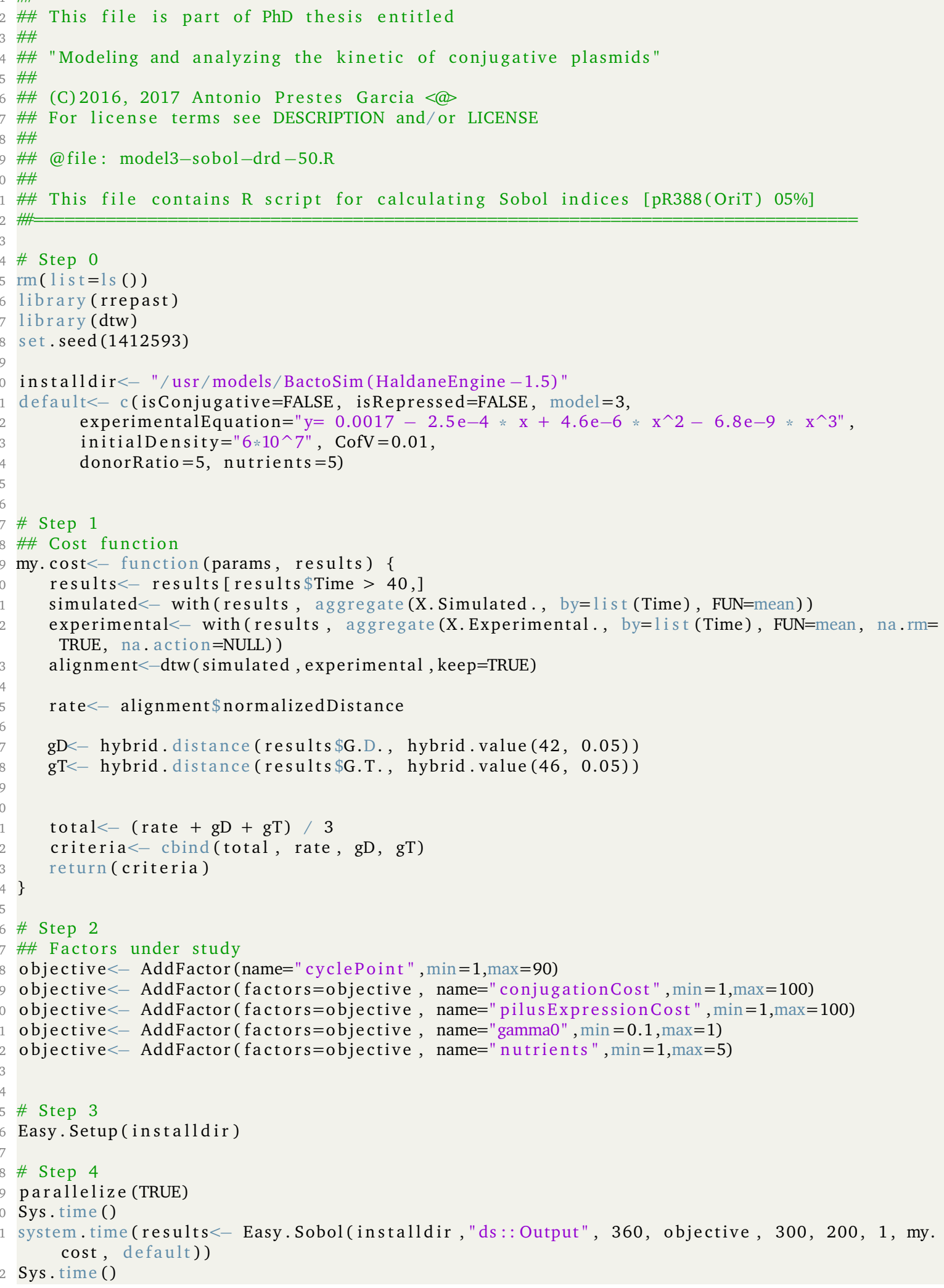

Figure A.13: The R code for applying Sobol GSA to pR388(OriT)/5\%. 


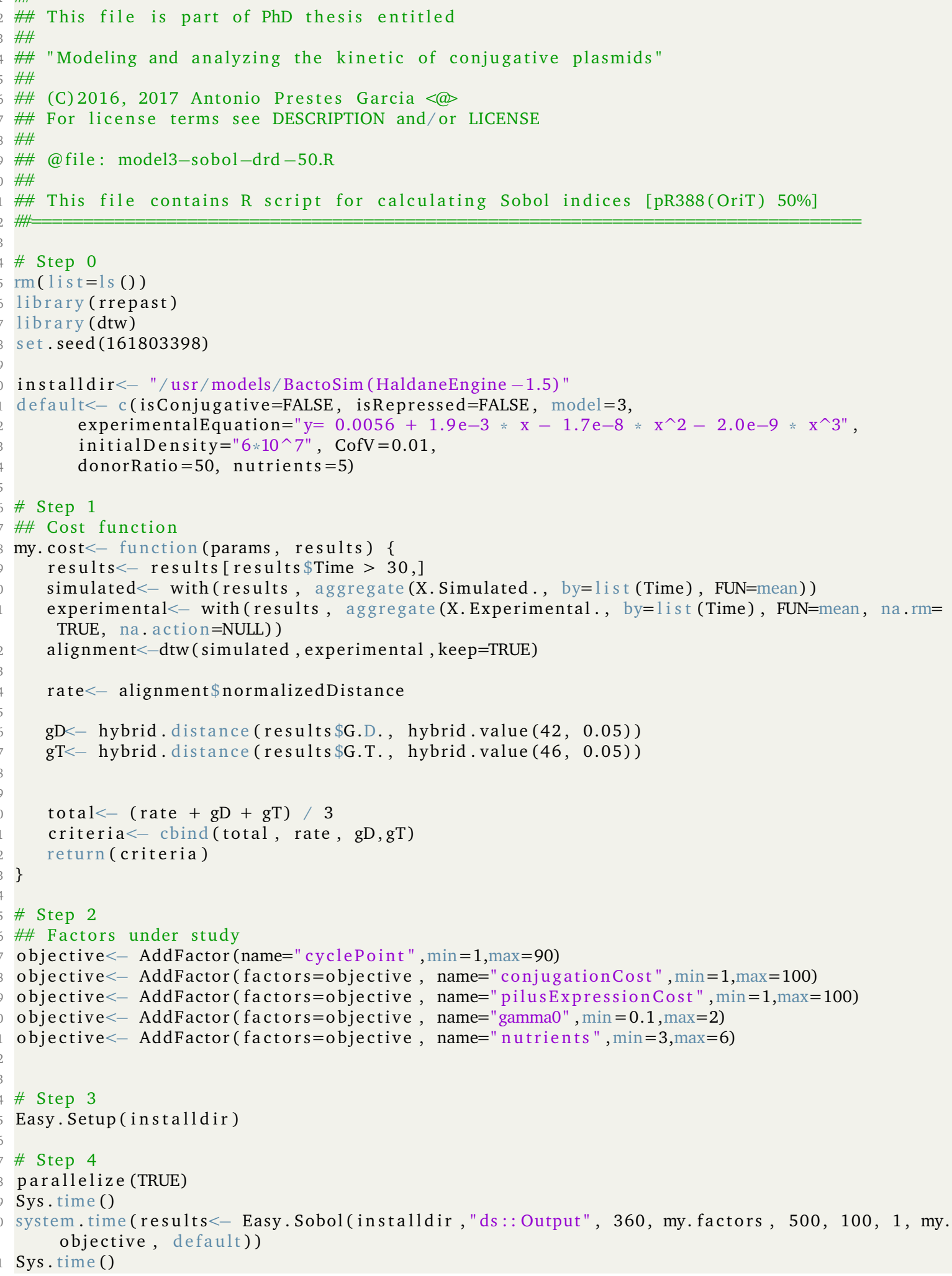

Figure A.14: The R code for applying Sobol GSA to pR388(OriT)/50\%. 